Aus der Klinik für Klinische Neurophysiologie

(Prof. Dr. med. W. Paulus)

im Zentrum Psychosoziale Medizin

der Medizinischen Fakultät der Universität Göttingen

\title{
Das Belastungserleben erwachsener Kinder von Parkinsonpatienten in Abhängigkeit von der Schlafqualität und dem Beziehungserleben
}

\author{
INAUGURAL - DISSERTATION \\ zur Erlangung des Doktorgrades \\ der Medizinischen Fakultät der \\ Georg-August-Universität zu Göttingen \\ vorgelegt von \\ Theresia Jacob, geb. Janssen \\ aus \\ Göttingen
}

Göttingen 2015 
I. Berichterstatter/in: Prof. Dr. med. S. Happe

II. Berichterstatter/in: Prof. Dr. phil. G. Reich

III. Berichterstatter/in: Prof. Dr. hum. biol. M. Schön

Tag der mündlichen Prüfung: $\quad$ 13.07.2016 


\section{Inhaltsverzeichnis}

Abbildungsverzeichnis ...................................................................................................................................... V

Tabellenverzeichnis ...................................................................................................................... VIII

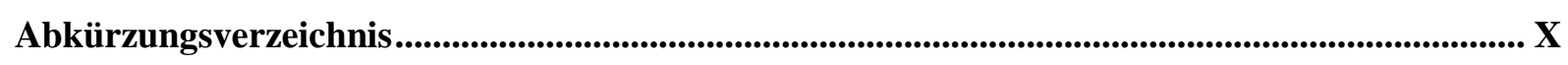

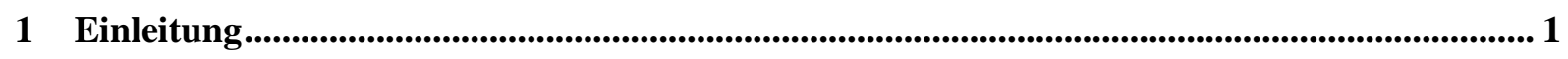

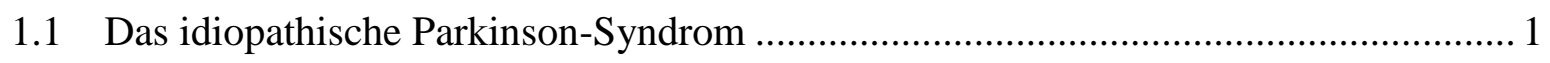

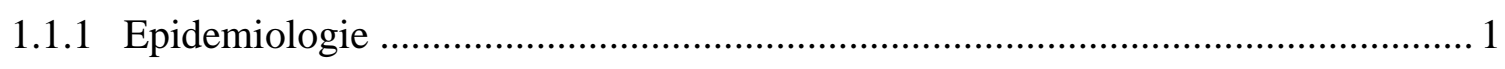

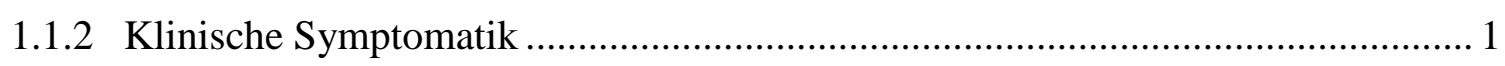

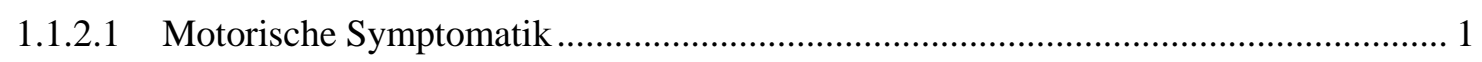

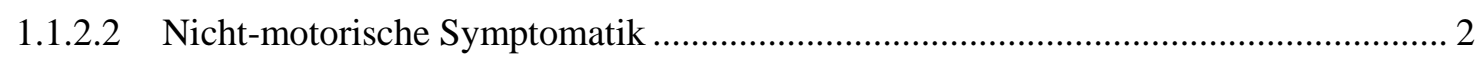

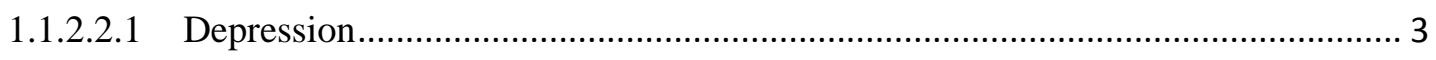

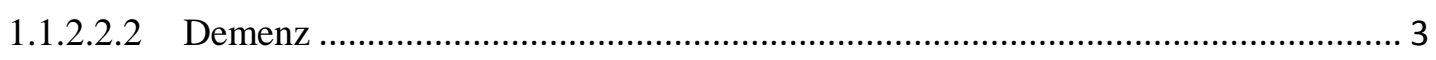

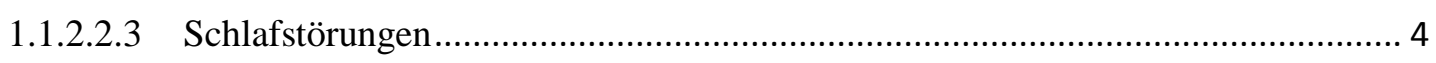

1.1.2.2.4 Andere nicht-motorische Symptome........................................................... 5

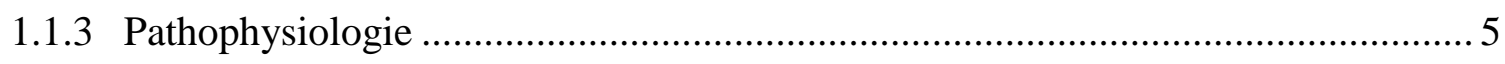

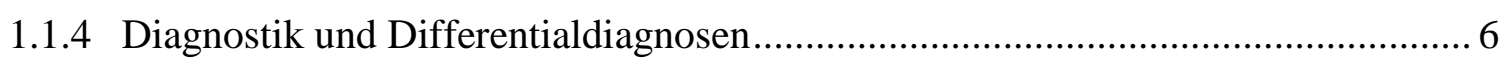

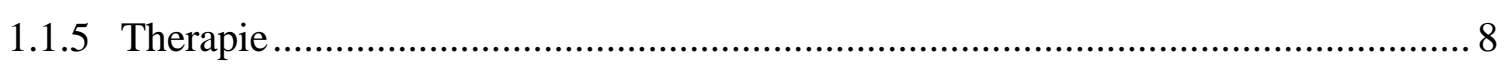

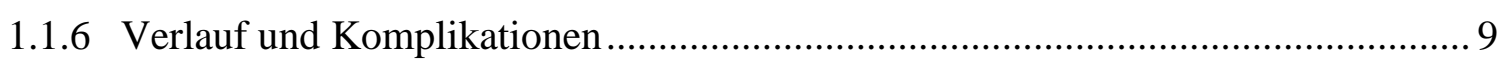

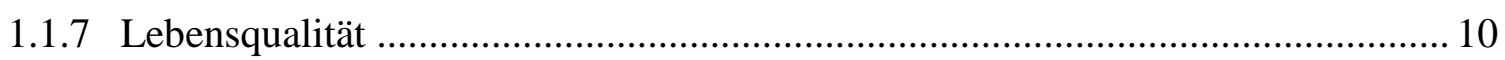

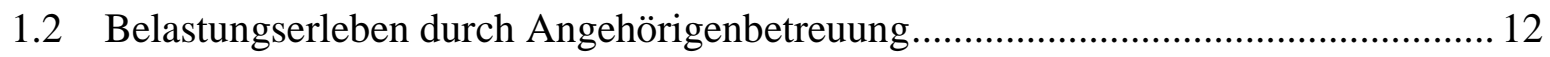

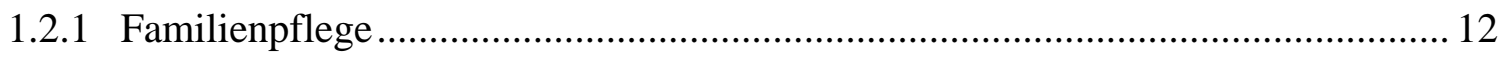

1.2.2 Belastungserleben durch die Pflegesituation.................................................. 14

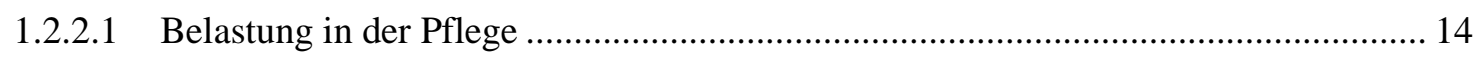

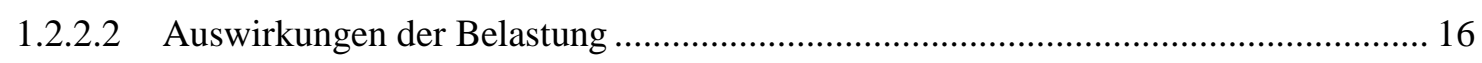

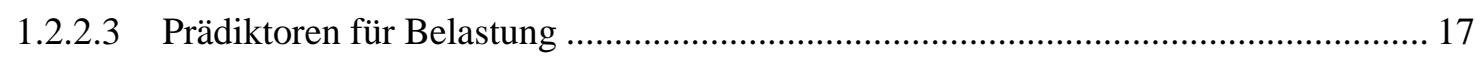

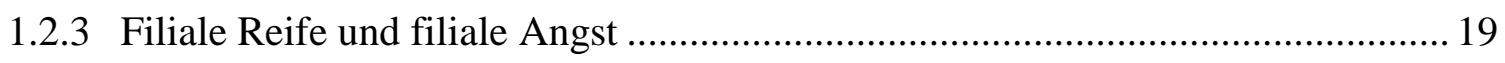

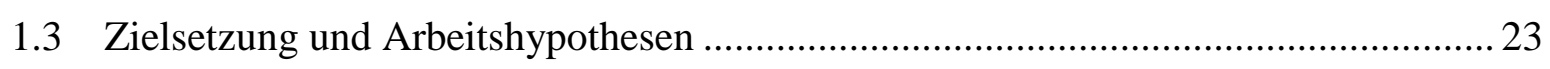

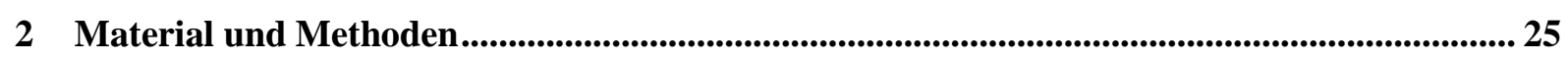

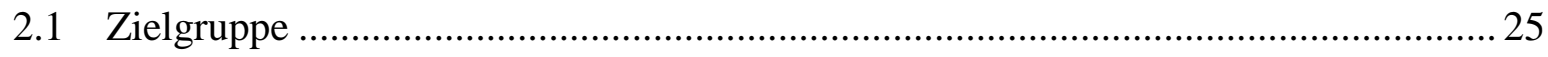

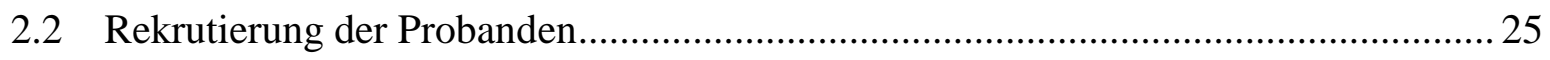

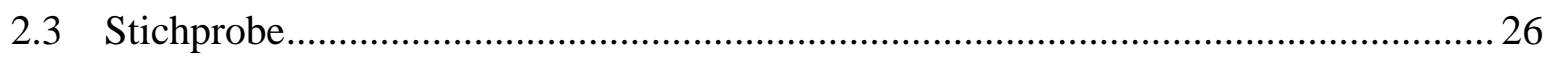

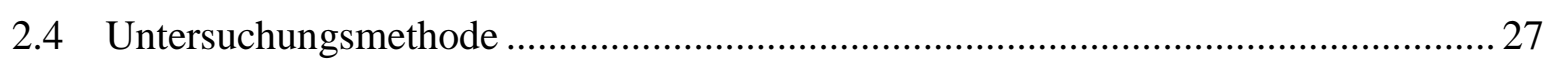

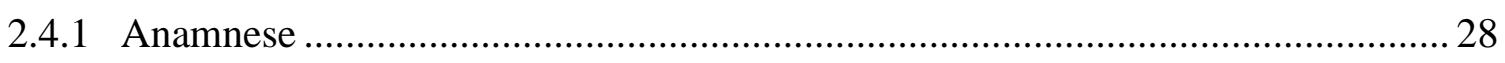

2.4.2 Stadienbestimmung des Parkinson-Syndroms nach Hoehn \& Yahr .................... 29

2.4.3 Unified Parkinson's Disease Rating Scale motor score (UPDRS Motorskala) ..... 30

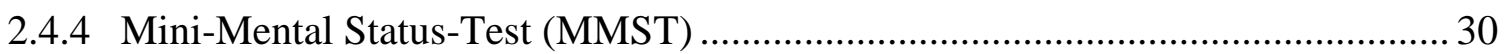


2.4.5 Geriatrische Depressionsskala nach Yesavage (GDS) ...................................... 31

2.4.6 10-Item Center for Epidemiological Studies Depression Scale (CESD-10) ......... 31

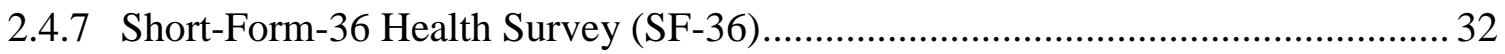

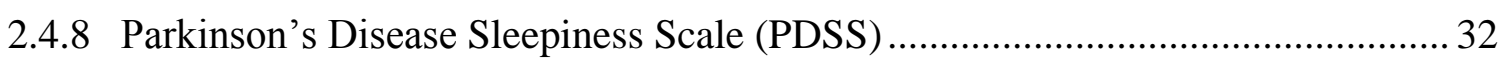

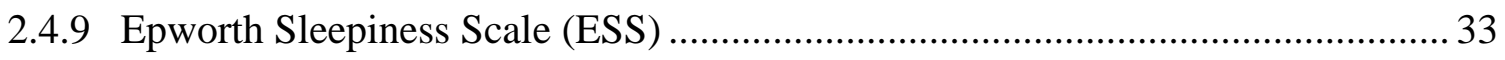

2.4.10 Pittsburgh Sleep Quality Index (PSQI) ............................................................ 33

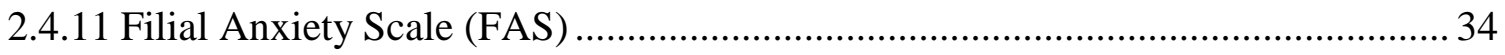

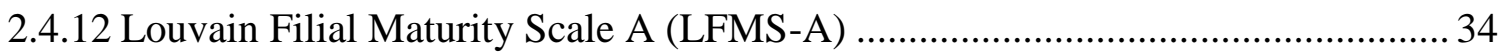

2.4.13 Fragebogen zur Belastung durch die Betreuung …......................................... 35

2.4.14 Nurses' Observation Scale for Geriatric Patients (NOSGER II) ........................... 35

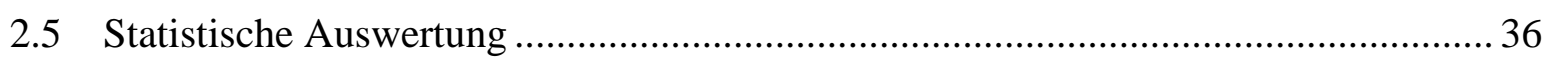

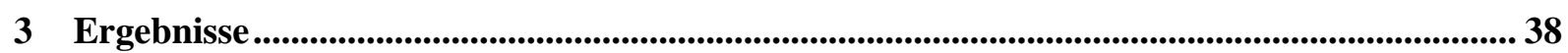

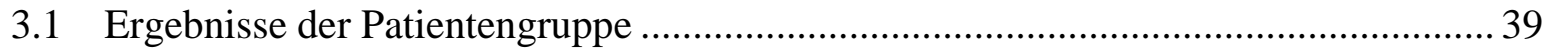

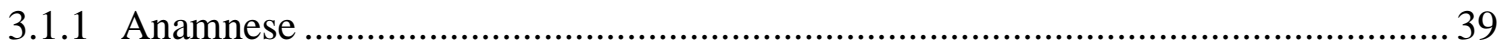

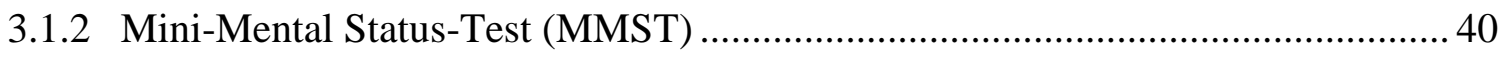

3.1.3 Geriatrische Depressionsskala nach Yesavage (GDS) ...................................... 40

3.1.4 10-Item Center for Epidemiological Studies Depression Scale (CESD-10) ......... 40

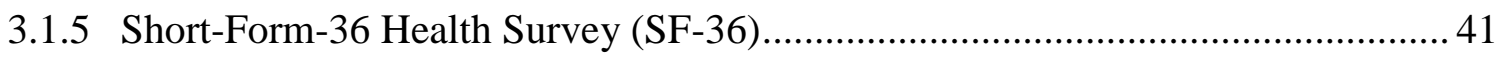

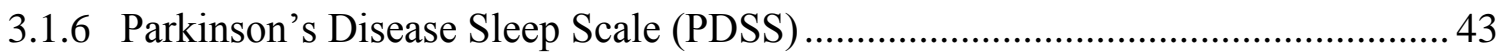

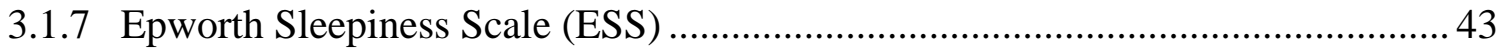

3.1.8 Pittsburgh Sleep Quality Index (PSQI) ....................................................... 44

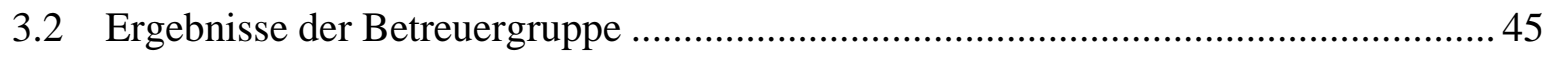

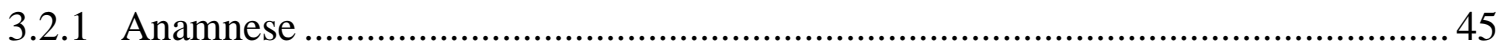

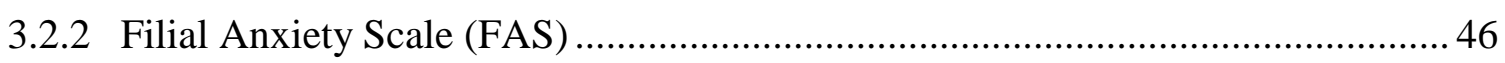

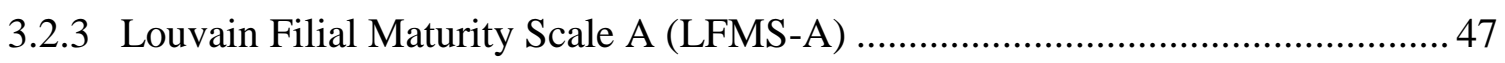

3.2.4 Fragebogen zur Belastung durch die Betreuung ................................................. 48

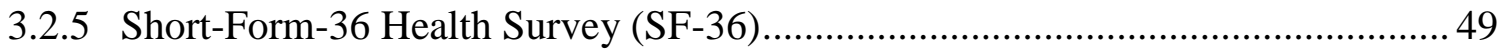

3.2.6 10-Item Center for Epidemiological Studies Depression Scale (CESD-10) ......... 50

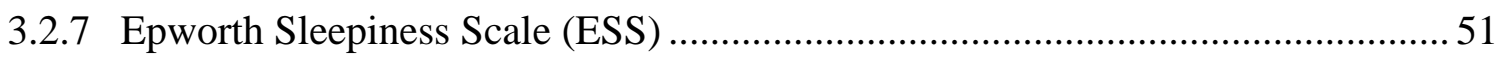

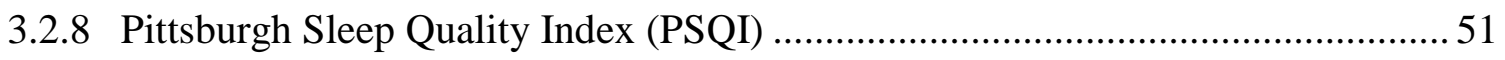

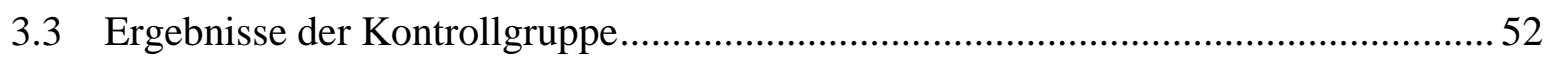

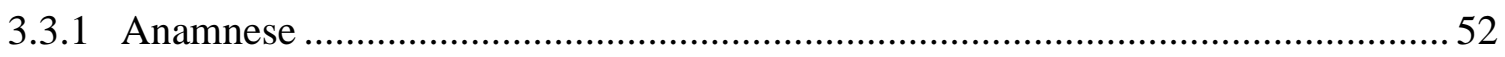

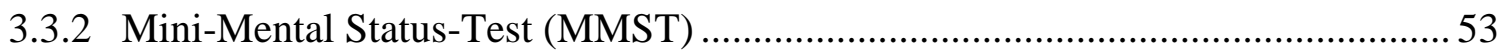

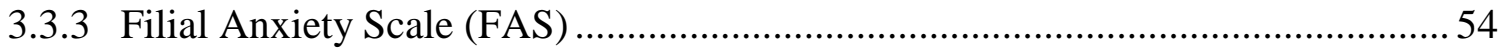

3.3.4 Louvain Filial Maturity Scale A (LFMS-A) …............................................... 54

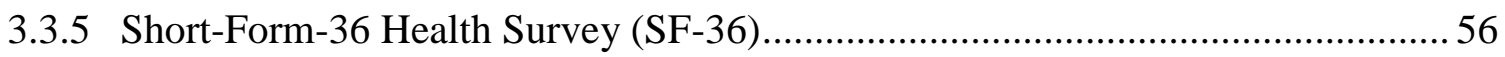

3.3.6 10-Item Center for Epidemiological Studies Depression Scale (CESD-10) ......... 57

3.3.7 Nurses' Observation Scale for Geriatric Patients (NOSGER II) ........................... 58 


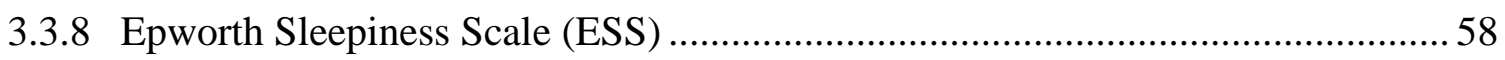

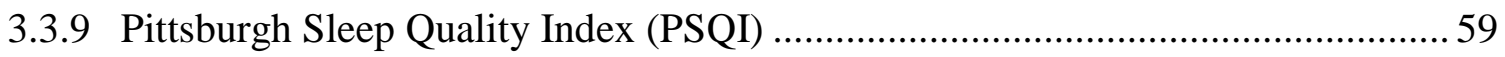

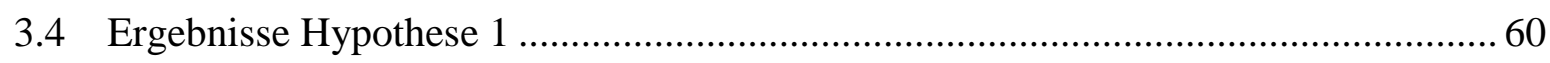

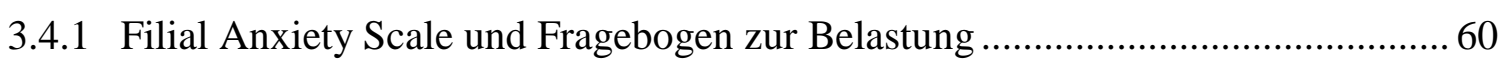

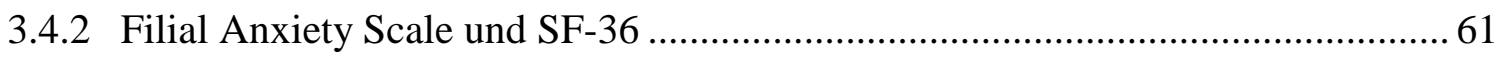

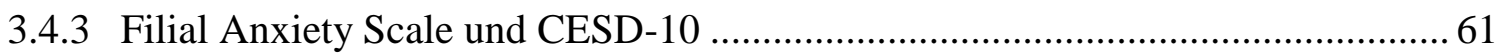

3.4.4 Filiale-Reife-Bogen und Fragebogen zur Belastung ......................................... 62

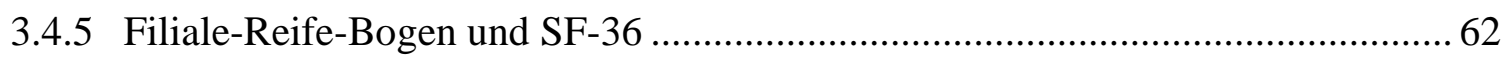

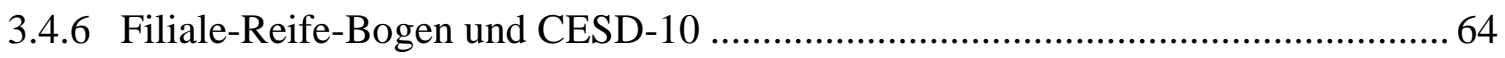

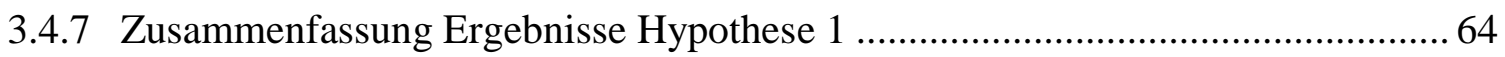

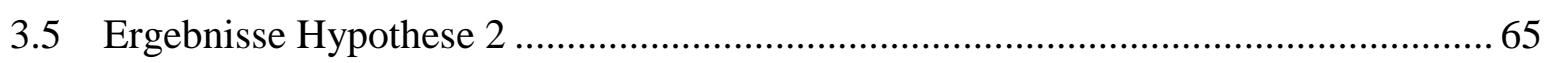

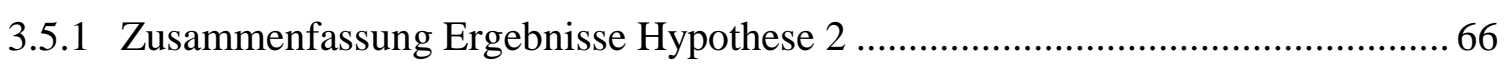

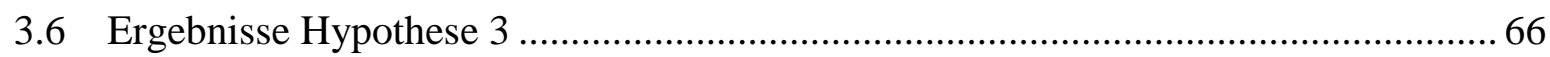

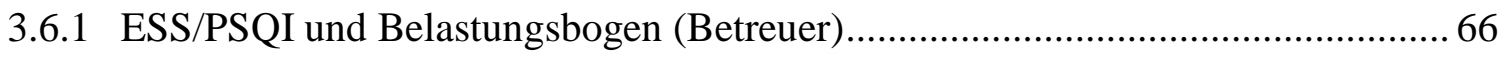

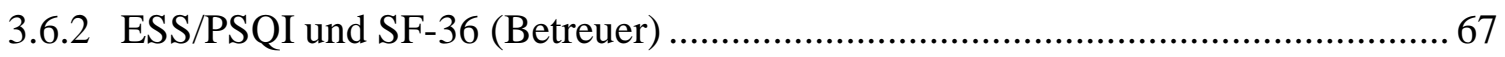

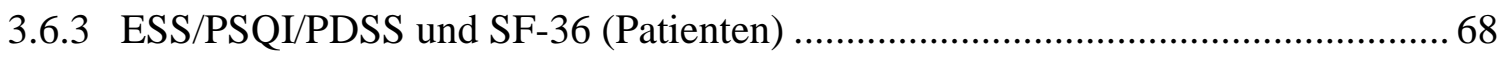

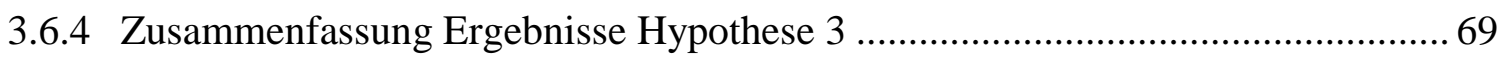

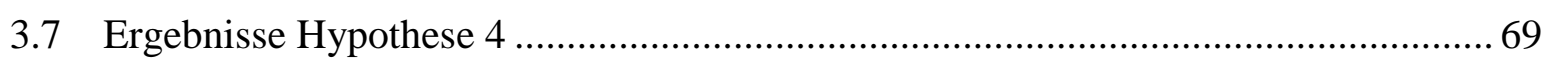

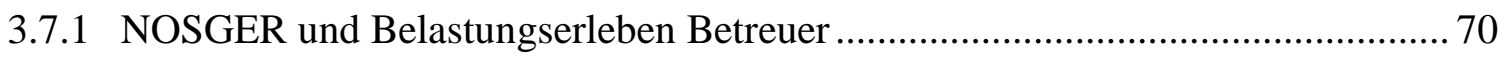

3.7.2 NOSGER und UPDRS, H\&Y, Erkrankungsdauer und Pflegestufe ....................... 71

3.7.3 Belastungsbogen und SF-36, GDS und CESD-10 (Patienten) ............................. 71

3.7.4 Belastungsbogen und UPDRS, H\&Y, Erkrankungsdauer und Pflegestufe........... 73

3.7.5 Zusammenfassung Ergebnisse Hypothese 4 ................................................... 73

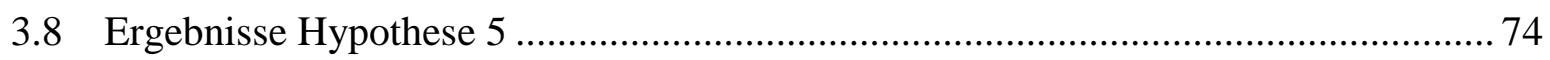

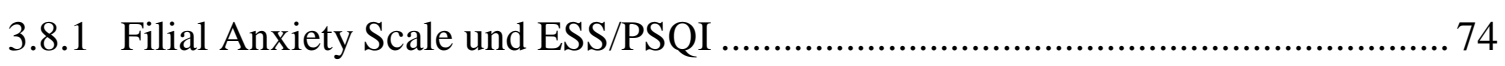

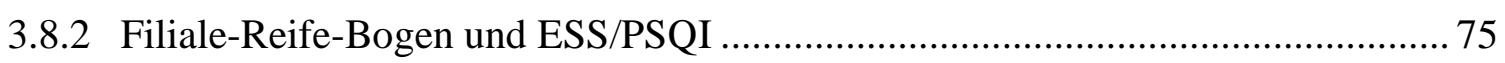

3.8.3 Zusammenfassung Ergebnisse Hypothese 5 …............................................ 75

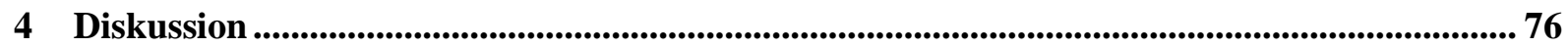

4.1 Diskussion Fragebögen Patientengruppe .......................................................... 76

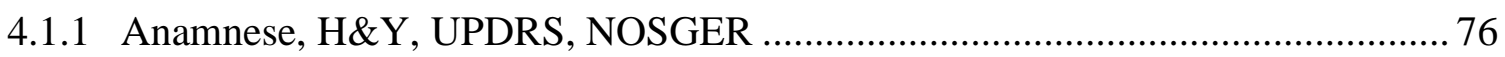

4.1.2 Geriatrische Depressionsskala nach Yesavage (GDS) und 10-Item Center for Epidemiological Studies Depression Scale (CESD-10) ...................................... 77

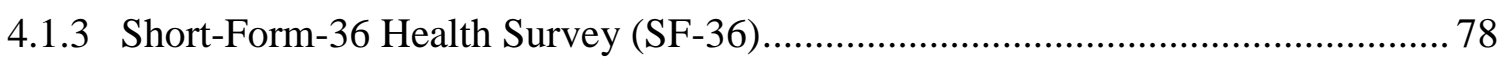

4.1.4 Parkinson's Disease Sleep Scale (PDSS) ............................................................. 79

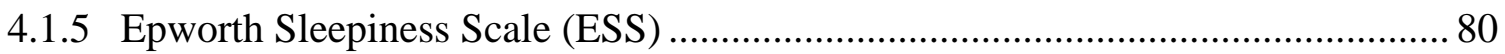

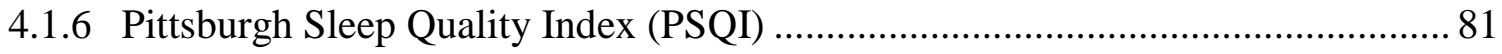

4.2 Diskussion Fragebögen Betreuergruppe und Kontrollgruppe .................................. 82

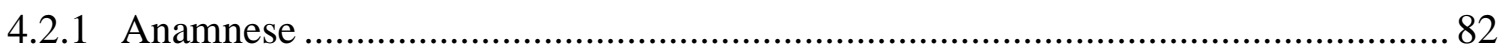




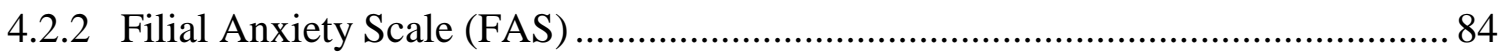

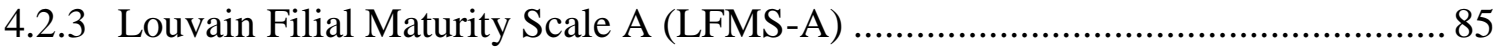

4.2.4 Fragebogen zur Belastung durch die Betreuung ….......................................... 87

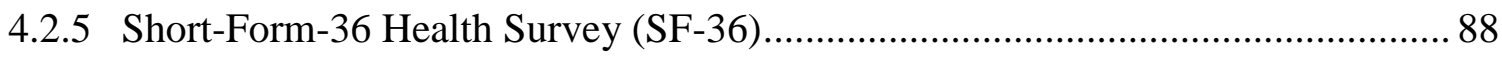

4.2.6 10-Item Center for Epidemiological Studies Depression Scale (CESD-10) ......... 90

4.2.7 Epworth Sleepiness Scale (ESS) und Pittsburgh Sleep Quality Index (PSQI) ..... 92

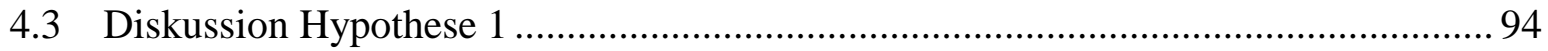

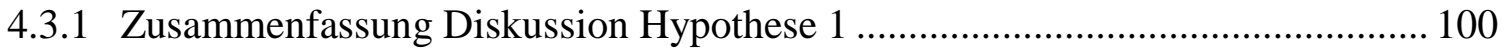

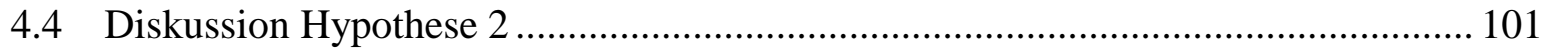

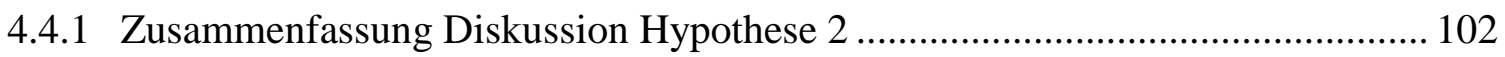

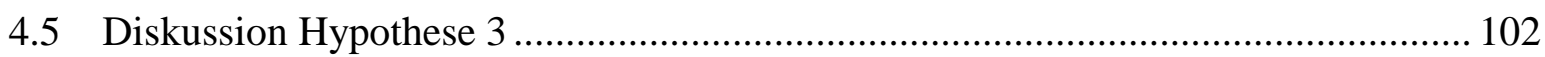

4.5.1 Zusammenfassung Diskussion Hypothese 3 …............................................... 106

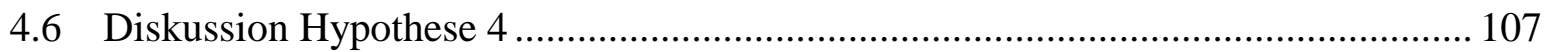

4.6.1 Zusammenfassung Diskussion Hypothese 4 .................................................... 109

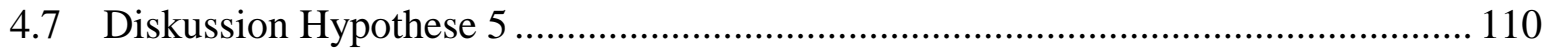

4.7.1 Zusammenfassung Diskussion Hypothese 5 ................................................. 113

5 Zusammenfassung........................................................................................................................... 114

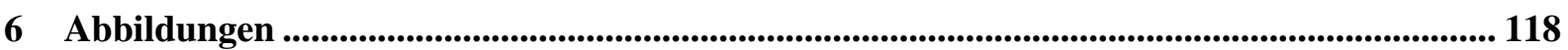

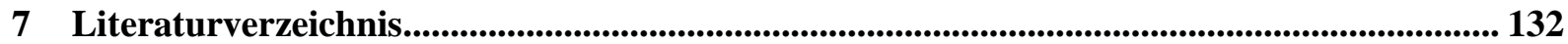




\section{Abbildungsverzeichnis}

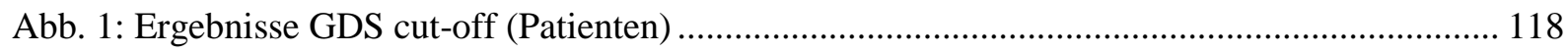

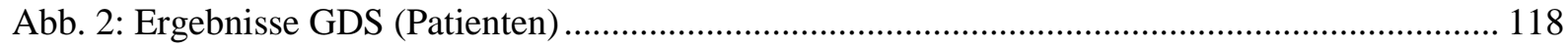

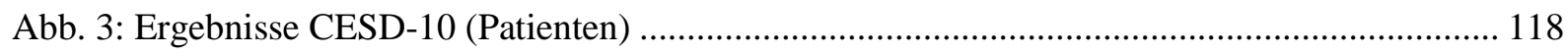

Abb. 4: Ergebnisse CESD-10 (männl./ weibl. Patienten)............................................................... 118

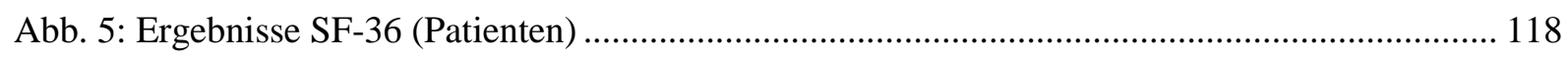

Abb. 6: Ergebnisse SF-36 (männl. Patienten) .............................................................................. 118

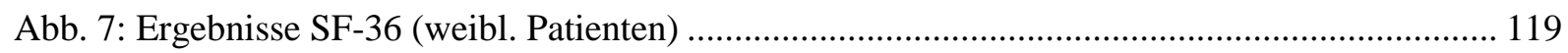

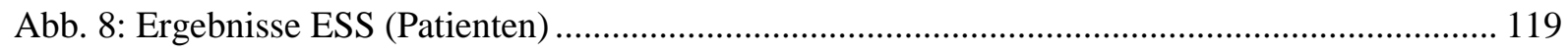

Abb. 9: Tabellarische Darstellung der Ergebnisse PSQI-Unterskalen (Patienten)............................. 119

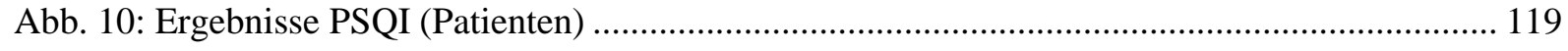

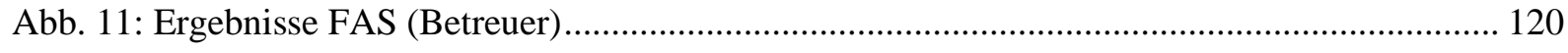

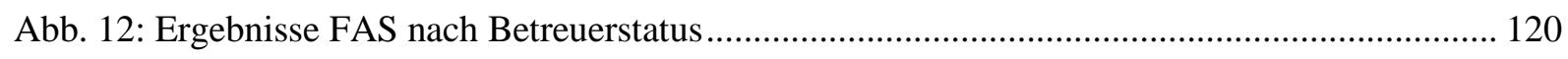

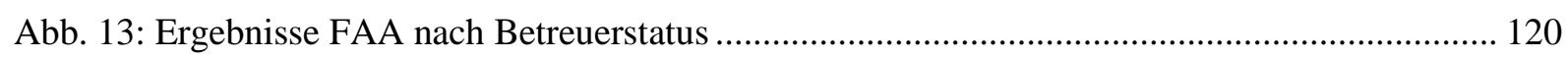

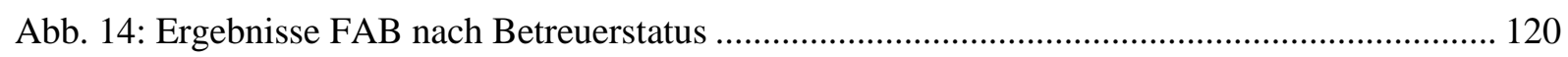

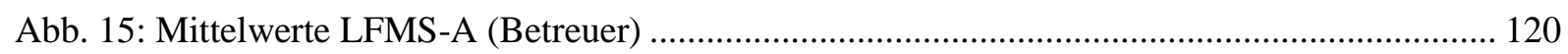

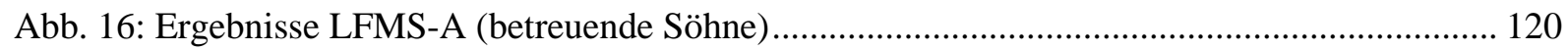

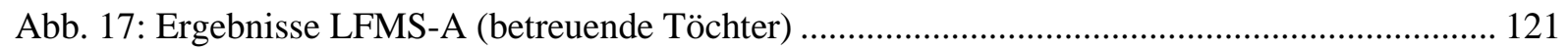

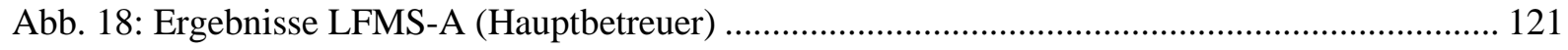

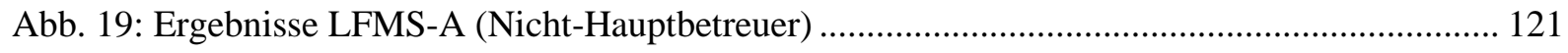

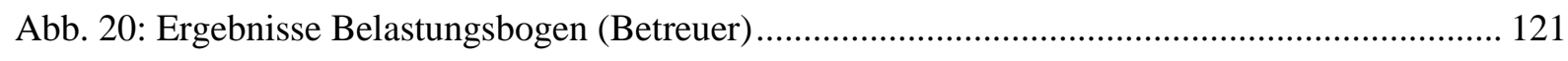

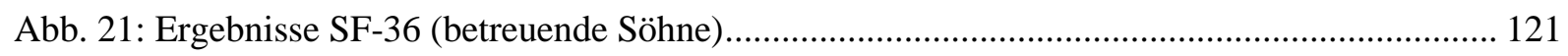

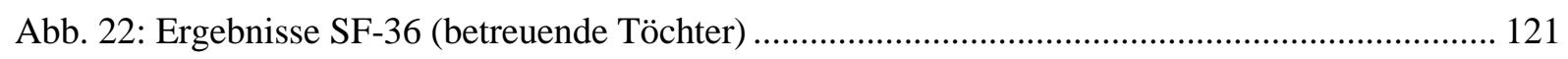

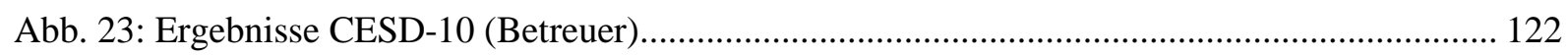

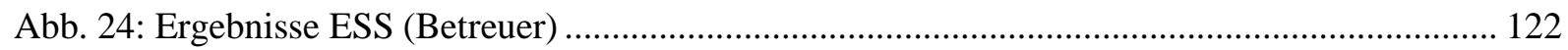

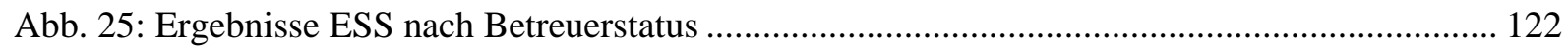

Abb. 26: Tabellarische Darstellung der Ergebnisse PSQI-Unterskalen (Betreuer)............................. 122

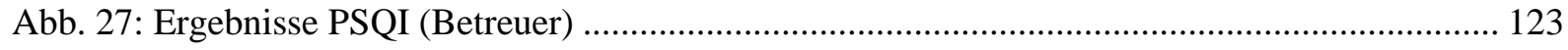

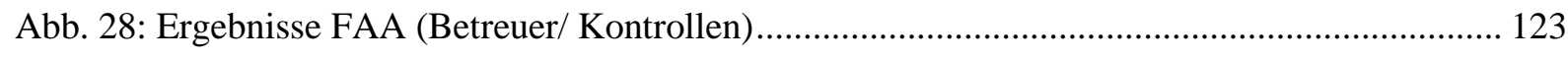

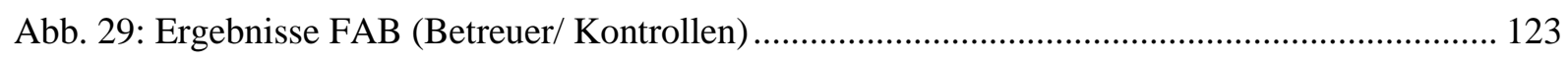

Abb. 30: Ergebnisse LFMS-A (männl. Kontrollen) …................................................................ 123

Abb. 31: Ergebnisse LFMS-A (weibl. Kontrollen) ......................................................................... 123

Abb. 32: Ergebnisse LFMS-A (männl. Betreuer/ Kontrollen) …......................................................... 123

Abb. 33: Ergebnisse LFMS-A (weibl. Betreuer/ Kontrollen) ........................................................... 124

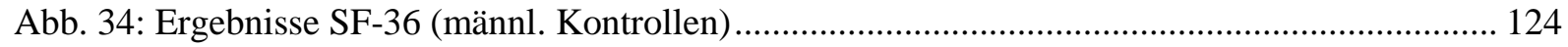

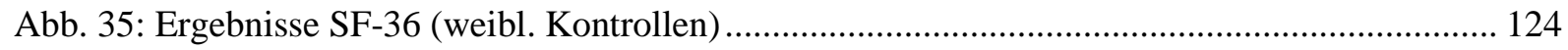




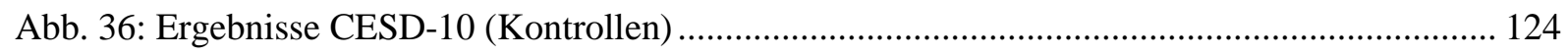

Abb. 37: Ergebnisse CESD-10 (Betreuer/ Kontrollen) ….............................................................. 124

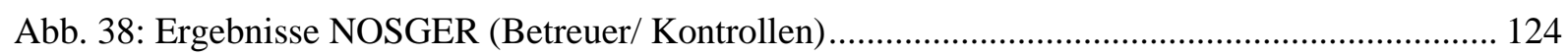

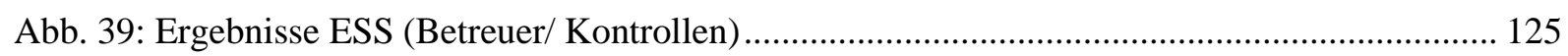

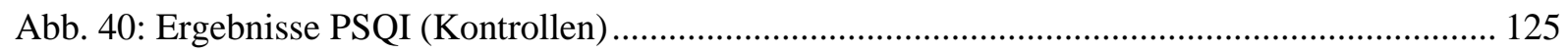

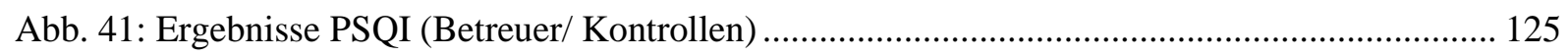

Abb. 42: Korrelation Belastungsbogen mit FAB (Betreuer) ........................................................... 125

Abb. 43: Korrelation Belastungsbogen mit FO der LFMS-A (Betreuer) .......................................... 125

Abb. 44: Tabellarische Darstellung der Korrelationen LFMS-A und SF-36 (betreuende Söhne) ...... 126

Abb. 45: Tabellarische Darstellung der Korrelationen LFMS-A und SF-36 (betreuende Töchter).... 126

Abb. 46: Tabellarische Darstellung der Korrelationen PSQI-Unterskalen und SF-36 (Betreuer) ...... 126

Abb. 47: Tabellarische Darstellung der Korrelationen PSQI-Unterskalen und SF-36 (betreuende Söhne)

Abb. 48: Tabellarische Darstellung der Korrelationen PSQI-Unterskalen und SF-36 (betreuende Töchter)

Abb. 49: Korrelationen KöFu/ KöRo und PSQI (Betreuer) .............................................................. 127

Abb. 50: Korrelationen KöSch/ AlGes und PSQI (Betreuer)............................................................ 127

Abb. 51: Korrelationen Vital/ SoFu und PSQI (Betreuer) ............................................................. 128

Abb. 52: Korrelationen EmRo/ PsyWo und PSQI (Betreuer) ....................................................... 128

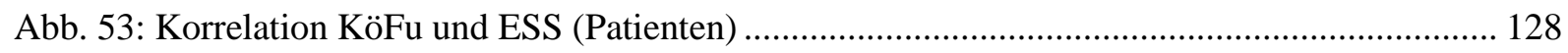

Abb. 54: Korrelationen KöFu/ KöRo und ESS (männl. Patienten) .................................................. 128

Abb. 55: Korrelationen AlGes/ EmRo und ESS (männl. Patienten) …….......................................... 128

Abb. 56: Korrelationen KöSch/ AlGes mit PSQI (Patienten) ........................................................ 128

Abb. 57: Korrelationen KöFu/ KöRo und PDSS (Patienten) ............................................................ 129

Abb. 58: Korrelationen KöSch/ AlGes und PDSS (Patienten)......................................................... 129

Abb. 59: Korrelationen Vital/ SoFu und PDSS (Patienten) ............................................................. 129

Abb. 60: Korrelationen EmRo und PsyWo und PDSS (Patienten) .................................................... 129

Abb. 61: Korrelation Belastungsbogen und NOSGER (Betreuer) ................................................... 129

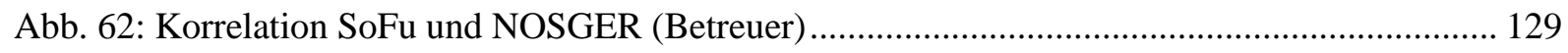

Abb. 63: Korrelation EmRo und NOSGER (Betreuer) ................................................................. 130

Abb. 64: Korrelationen KöFu/ KöRo (Patienten) und Belastung (Betreuer) ...................................... 130

Abb. 65: Korrelationen KöSch/ AlGes (Patienten) und Belastung (Betreuer) .................................... 130

Abb. 66: Korrelationen Vital/ SoFu (Patienten) und Belastung (Betreuer)........................................ 130

Abb. 67: Korrelation GDS (Patienten) und Belastung (Betreuer)...................................................... 130

Abb. 68: Korrelation CESD-10 (Patienten) und Belastung (Betreuer) .............................................. 130

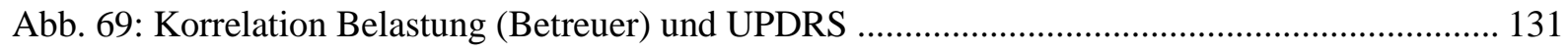

Abb. 70: Korrelation Belastung (Betreuer) und H\&Y-Stadium......................................................... 131 
Abb. 71: Korrelation FAA und PSQI-Unterscore 5 (Betreuer)

Abb. 72: Korrelation FAB und PSQI-Unterscore 5 (Betreuer) 


\section{Tabellenverzeichnis}

Tabelle 1: Diagnosekriterien des idiopathischen Parkinson-Syndroms nach der UK Parkinson's

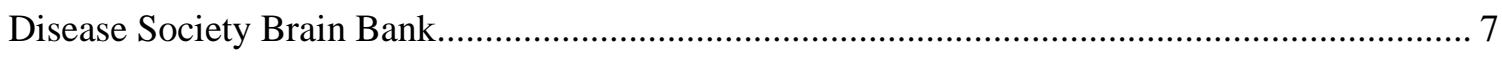

Tabelle 2: Ergebnisse der Geriatrischen Depressionsskala nach Yesavage ........................................ 40

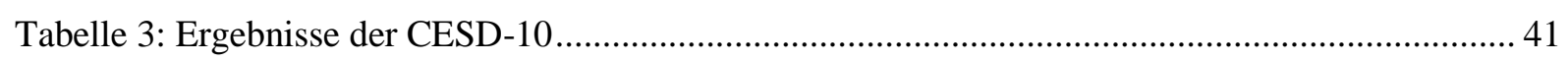

Tabelle 4: Mittelwerte und Standardabweichungen des SF-36 im Vergleich mit der Normstichprobe des Bundes-Gesundheitssurveys 1998 (Ellert und Bellach 1999) …................................................ 42

Tabelle 5: Ergebnisse der Parkinson's Disease Sleep Scale................................................................... 43

Tabelle 6: Ergebnisse der ESS im Vergleich mit den Werten von Bloch et al. .................................... 44

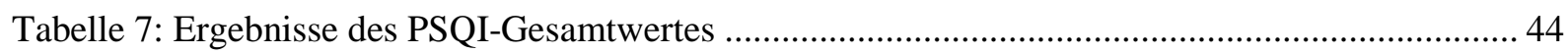

Tabelle 8: Mittelwerte und Standardabweichungen der Filial Anxiety Scale im Vergleich mit den

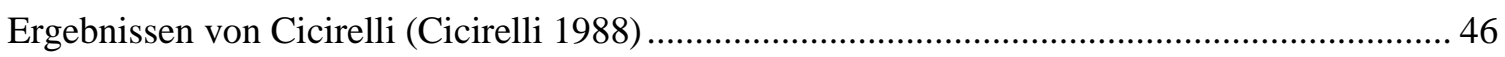

Tabelle 9: Mittelwerte und Standardabweichungen des Filiale-Reife-Bogens (LFMS-A) im Vergleich mit den Ergebnissen zweier Vorstudien (Stiens 2000 [G.S.], Linkersdörfer 2006 [V.L.]) .......... 47

Tabelle 10: Extremwerte, Mittelwerte und Standardabweichungen des Belastungsbogens im Vergleich mit den Ergebnissen der Vorstudie von Stiens (Stiens 2000 [G.S.]) 48

Tabelle 11: Mittelwerte und Standardabweichungen des SF-36 im Vergleich mit der Normstichprobe des Bundes-Gesundheitssurveys 1998 (Ellert und Bellach 1999) ................................................ 49

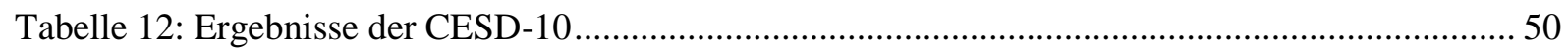

Tabelle 13: Ergebnisse der ESS im Vergleich mit den Werten von Bloch et al. (1999) ....................... 51

Tabelle 14: Ergebnisse des PSQI im Vergleich mit den Werten von Zeitlhofer et al. (2000) .............. 52

Tabelle 15: Mittelwerte und Standardabweichungen der Filial Anxiety Scale der Kontrollpersonen im Vergleich mit den Ergebnissen der Studiengruppe.

Tabelle 16: Vergleich der Mittelwerte und Standardabweichungen des Filiale-Reife-Bogens (LFMS-

A) zwischen der Studien- und Kontrollgruppe 55

Tabelle 17: Mittelwerte und Standardabweichungen des SF-36 der Kontrollgruppe im Vergleich mit den Ergebnissen der Studiengruppe

Tabelle 18: Vergleich der Ergebnisse der CESD-10 zwischen der Gruppe der Betreuer und

Kontrollpersonen

Tabelle 19: Mittelwerte und Standardabweichungen der NOSGER der Kontrollgruppe im Vergleich

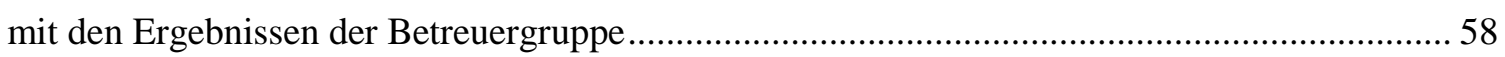

Tabelle 20: Vergleich der Ergebnisse der ESS zwischen der Studien- und Kontrollgruppe. 59

Tabelle 21: Ergebnisse der PSQI-Gesamtwerte der Kontrollpersonen im Vergleich mit den Ergebnissen der Studiengruppe 59

Tabelle 22: Korrelation zwischen Filial Anxiety Scale und dem Belastungsbogen 60

Tabelle 23: Korrelation zwischen der Filial Anxiety Scale und dem SF-36 61 
Tabelle 24: Korrelation zwischen Filial Anxiety Scale und der CESD-10 .

Tabelle 25: Korrelation zwischen der LFMS-A und dem Belastungsbogen ......................................... 62

Tabelle 26: Korrelation zwischen der LFMS-A und dem SF-36 für die Gesamtstichprobe ................. 63

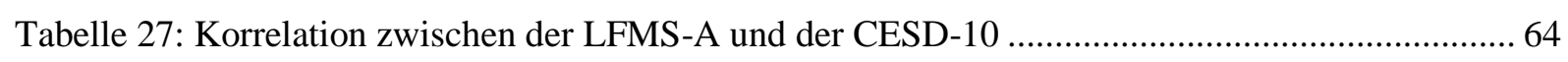

Tabelle 28: Vergleich der Korrelationen der Filial Anxiety Scale mit dem Belastungsbogen und der

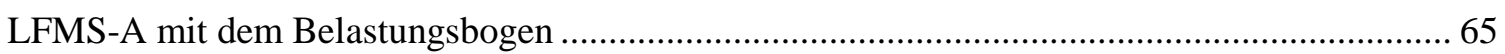

Tabelle 29: Korrelationen der ESS und der PSQI der Betreuer mit dem Belastungsbogen ................... 66

Tabelle 30: Korrelation zwischen dem ESS/PSQI-Gesamtwert und dem SF-36 (Betreuer)................. 67

Tabelle 31: Korrelation zwischen der ESS und dem SF-36, dem PSQI-Gesamtwert und dem SF-36

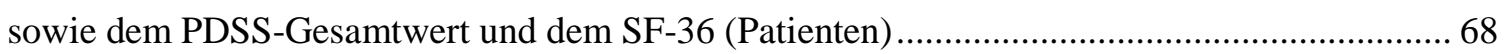

Tabelle 32: Korrelationen der NOSGER mit dem Belastungsbogen, dem SF-36 und der CESD-10 der Betreuer .

Tabelle 33: Korrelationen der NOSGER mit dem UDPRS-Score, dem Hoehn-Yahr-Stadium, der Erkrankungsdauer und der Pflegestufe der Patienten .

Tabelle 34: Korrelationen des Belastungsbogens der Betreuer mit dem SF-36, der GDS und der CESD-10 der Patienten

Tabelle 35: Korrelationen des Belastungsbogens der Betreuer mit dem UDPRS-Motorscore, dem

Hoehn-Yahr-Stadium, der Erkrankungsdauer und der Pflegestufe der Patienten 73

Tabelle 36: Korrelationen der Filial Anxiety Scale mit der ESS und der PSQI der Betreuer..... 74

Tabelle 37: Korrelationen des Filiale-Reife-Bogens mit der ESS und der PSQI der Betreuer 75 


\section{Abkürzungsverzeichnis}

$\begin{array}{ll}\text { ADL } & \text { Aktivitäten des täglichen Lebens } \\ \text { AlGes } & \text { allgemeine Gesundheit } \\ \text { CESD } & \text { Center for Epidemiological Studies Depression Scale } \\ \text { elt. } & \text { elterlich } \\ \text { EmRo } & \text { emotionale Rollenfunktion } \\ \text { ESS } & \text { Epworth Sleepiness Scale }\end{array}$

FA filiale Autonomie

FAA Filial Anxiety Scale A

FAB Filial Anxiety Scale B

FAS Filial Anxiety Scale

FH filiale Hilfe

FHN filiale Hilfsbereitschaft

fil. filial

FL filiale Liebe

FO filiales Verpflichtungsgefühl

FS familiäre Solidarität

GDS Geriatrische Depressionsskala nach Yesavage

G.S. Gerthild Stiens

H\&Y Hoehn \& Yahr

IADL instrumentelle Aktivitäten des täglichen Lebens

$\mathrm{KöFu \quad körperliche} \mathrm{Funktionsfähigkeit}$

KöRo körperliche Rollenfunktion

KöSch körperliche Schmerzen

LFMS-A Louvain Filial Maturity Scale A

männl. männlich

MMST Mini-Mental Status Test

NOSGER Nurses` Observation Scale for Geriatric Patients

Pat. Patient

PC elterliche Besorgnis

PDSS Parkinson's Disease Sleep Scale

PSQI Pittsburgh Sleep Quality Index

PsyWo psychisches Wohlbefinden

SD Standardabweichung

SF-36 Short-Form-36 Health Survey

$\mathrm{SoFu} \quad$ soziale Funktionsfähigkeit 
soz.

UPDRS

Vital

V.L.

weibl. sozial

Unified Parkinson's Disease Rating Scale

Vitalität

Valeska Linkersdörfer

weiblich 


\section{Einleitung}

\subsection{Das idiopathische Parkinson-Syndrom}

Unter der Bezeichnung Parkinson-Syndrome werden eine Reihe unterschiedlicher hypokinetischhypertoner Krankheitsbilder mit den Kardinalsymptomen Rigor, Tremor und Akinese zusammengefasst. James Parkinson beschrieb im Jahre 1817 erstmals diesen Symptomenkomplex als shaking palsy (Parkinson 1817). Der weitaus größte Teil der Parkinson-Syndrome tritt idiopathisch auf und wird nach seinem Erstbeschreiber auch als Morbus Parkinson bezeichnet. Etwa zwanzig bis dreißig Prozent sind symptomatischer Genese (z.B. medikamenten-induziert, vaskulär, metabolisch) oder treten im Rahmen anderer neurodegenerativer Erkrankungen auf (Leitlinie Parkinson-Syndrome 2012; Munhoz et al. 2010; Schulz und Gasser 2007). In den letzten Jahren sind zudem eine ganze Reihe autosomal dominanter (z.B. PARK 1, 4, 8 und 17) und rezessiver Gene (z.B. PARK 2, 6, 7, 9, 14, 15) lokalisiert worden, die bei einem Teil der Patienten auf eine monogenetische Ursache und somit auf ein familiäres Parkinson-Syndrom hinweisen (Houlden und Singleton 2012). Darüber hinaus wurde eine Vielzahl an genetischen prädispositionierenden Faktoren lokalisiert, welche das Risiko für das Auftreten eines Parkinson-Syndroms erhöhen (Singleton et al. 2013).

\subsubsection{Epidemiologie}

Die Prävalenz des idiopathischen Parkinson-Syndroms in der deutschen Gesamtbevölkerung wird auf 100-200 pro 100.000 Einwohner geschätzt und ist somit nach der Alzheimerdemenz die zweithäufigste neurodegenerative Erkrankung in Deutschland (Schulz und Gasser 2007). Die Rate der jährlichen Neuerkrankungen liegt bei 16-19/100.000 Einwohnern (Twelves et al. 2003). Da mit steigendem Lebensalter die Inzidenz des idiopathischen Parkinson-Syndroms zunimmt, liegt die Prävalenz der Erkrankung in der Gruppe der über 65-jährigen Personen bei 1,8 Prozent (de Rijk et al. 2000; Schulz und Gasser 2007). Durch die Veränderungen der Altersstruktur der Bevölkerung ist so in den kommenden Jahren mit einer weiter ansteigenden Patientenzahl zu rechnen. Das durchschnittliche Manifestationsalter liegt zwischen 50 und 60 Jahren, 10\% der Patienten sind bei Erkrankungsbeginn jünger als 40 Jahre. Der Anteil des männlichen Geschlechts überwiegt leicht (Schulz und Gasser 2007).

\subsubsection{Klinische Symptomatik}

\subsubsection{Motorische Symptomatik}

Das idiopathische Parkinson-Syndrom ist durch Rigor, Tremor und Akinese gekennzeichnet und wird häufig von vegetativen Symptomen begleitet (Leitlinie Parkinson-Syndrome 2012; Schulz und Gasser 
2007). Rigor und Tremor zeigen dabei typischerweise einen unilateralen Beginn. Im späteren Krankheitsverlauf kann durch eine Verminderung der Haltungs- und Stellreflexe eine posturale Instabilität mit daraus resultierender Sturzgefahr für den Patienten hinzukommen (Leitlinie ParkinsonSyndrome 2012; Nutt und Wooten 2005). Je nach vorherrschender Symptomatik kann zwischen dem akinetisch-rigiden, dem Tremordominanz- und dem Äquivalenztyp sowie der seltenen Variante eines monosymptomatischen Ruhetremors unterschieden werden (Leitlinie Parkinson-Syndrome 2012).

Der Rigor ist durch eine gleichzeitige Tonuserhöhung in Agonisten und Antagonisten gekennzeichnet und wird anfänglich häufig durch Schmerzen in Hüfte oder Schulter als altersbedingte Abnahme der allgemeinen körperlichen Funktionsfähigkeit fehlgedeutet (Nutt und Wooten 2005; Schulz und Gasser 2007). Das so genannte „Zahnradphänomen“ ist ebenso wie der gleichmäßig wächsernen Widerstand bei passiver Bewegung typisch für die Erkrankung.

Der affektiv verstärkbare Ruhetremor mit einer Frequenz von 4-6 Hz ist durch die simultane Aktivierung antagonistisch wirkender Muskeln bedingt und häufig an den distalen oberen Extremitäten zu beobachten (sog. „Pillendreher-Phänomen“). Bei Intentionsbewegungen nimmt er häufig ab (Leitlinie Parkinson-Syndrome 2012; Schulz und Gasser 2007).

Die Akinese beschreibt die verlangsamte Initiation und Ausführung von Willkürbewegungen (Bradykinese) und die allgemeine Verarmung an Begleitbewegungen wie beispielsweise das Mitschwingen der Arme beim Gehen (Hypokinese). Die Verlangsamung von Pronations-/ Supinationsbewegungen äußert sich als Bradydiadochokinese (Schulz und Gasser 2007).

Charakteristisch sind weiterhin eine gebundene Körperhaltung und ein gebeugtes, kleinschrittiges Gangbild mit akinetischer Starthemmung, Festinationen und sog. freezing-Effekten. Neben einer hypophonen, monoton verwaschenen Sprache ist zudem eine Hypomimie und Mikrographie kennzeichnend für das idiopathische Parkinson-Syndrom (Leitlinie Parkinson-Syndrome 2012; Nutt und Wooten 2005; Schulz und Gasser 2007).

\subsubsection{Nicht-motorische Symptomatik}

Im Gegensatz zu James Parkinsons Beobachtung, nach der die Sinne und die geistigen Fähigkeiten der Patienten von der Erkrankung verschont blieben, können neben den motorischen Symptomen eine Reihe neuropsychiatrischer und autonomer Symptome im Verlauf des idiopathischen ParkinsonSyndroms auftreten (Antonini et al. 2012; Khedr et al. 2013). Die Prävalenz und Schwere dieser nichtmotorischen Symptome nehmen mit Dauer der Erkrankung zu und beeinflussen in starkem Ausmaß sowohl die allgemeine Lebensqualität und die Progression der körperlichen Einschränkungen als auch den Zeitpunkt der Institutionalisierung der Parkinson-Patienten (vgl. Antonini et al. 2012; Poewe 2008). 


\subsection{Depression}

Das Auftreten einer Depression stellt eine der häufigsten psychiatrischen Komplikationen des idiopathischen Parkinson-Syndroms dar und betrifft im Krankheitsverlauf etwa $40 \%$ der Patienten (Khedr et al. 2013; Lemke 2008; Quin et al. 2009; Slaughter et al. 2001). Nicht selten wird von wiederholten depressiven Episoden schon Jahre vor der klinischen Manifestation des ParkinsonSyndroms berichtet (Merschdorf et al. 2003). Die Ausprägung depressiver Symptome ist individuell sehr unterschiedlich, beinhaltet in der Regel jedoch neben einer allgemeinen psychomotorischen Verlangsamung und erhöhten Reizbarkeit des Patienten unterschiedliche Level von Dysphorie und Anhedonie, einen Verlust an Interessen und Initiative sowie Apathie (Lemke 2008, Poewe 2008). Im Vergleich zu Patienten mit einer endogenen Depression (major depression) zeigen depressive Parkinsonpatienten eine relative Abwesenheit von Schuld- und Versagensgefühlen und trotz vergleichsweise häufigen Selbstmordgedanken eine geringere Selbstmordrate (Lemke 2008; Slaughter et al. 2001). Die Diagnose einer Depression im Verlauf des idiopathischen Parkinson-Syndroms basiert u.a. auf einem subjektiv erlebten Gefühl der Leere und Hoffnungslosigkeit, einer allgemeinen Freudlosigkeit sowie einer verminderten Reaktion auf emotionale Stimuli (Lemke 2008).

Das Auftreten und die Schwere der depressiven Symptome korreliert dabei weder mit der Erkrankungsdauer noch mit dem Grad der motorischen Behinderung (Schulz und Gasser 2007). Daher kann die Depression nicht als sekundäre Reaktion auf die motorischen Defizite gewertet werden (Lemke 2008). Sie erfordert jedoch häufig eine antidepressive Therapie.

\subsection{Demenz}

Bei der Mehrzahl der Parkinson-Patienten können schon in den frühen Krankheitsstadien mittels spezifischer neuropsychiatrischer Untersuchungen geringe kognitive Defizite festgestellt werden, die sich in 20 bis $40 \%$ der Fälle zu einer klinisch manifesten Demenz weiter entwickeln (Bosboom et al. 2004; Khedr et al. 2013; Poewe 2008; Schulz und Gasser 2007). Im Vordergrund stehen hierbei zunehmende Defizite bei der Planung und Ausführung zielgerichteter und situationsgerechter Handlungen, was sich besonders auf die Aktivitäten des täglichen Lebens einschränkend auswirkt. Zudem zeigen die Patienten Defizite in den visuell-räumlichen Funktionen (Poewe 2008). Die allgemeine Aufmerksamkeit ist ebenso wie die Fähigkeit, Gedächtnisinhalte richtig abzurufen, vermindert (Schulz und Gasser 2007). Die Schwere der Symptomatik grenzt die Parkinson-Demenz von den geringfügigeren kognitiven Defiziten der nicht-dementen Parkinson-Patienten ab. Visuelle Halluzinationen und psychotische Episoden können als weitere Symptome der Parkinson-Demenz auftreten (Bosboom et al. 2004).

Die Entwicklung einer Demenz im Krankheitsverlauf des idiopathischen Parkinson-Syndroms ist mit einem vergleichsweise schnelleren Voranschreiten der körperlichen Behinderung sowie einer höheren 
Sterblichkeit der Patienten assoziiert und geht mit einem erhöhten Risiko einer Heimeinweisung einher (Poewe 2008).

\subsection{Schlafstörungen}

Schlafstörungen gehören zu den häufigsten nicht-motorischen Symptomen und kommen im Verlauf der Parkinson-Erkrankung bei nahezu allen Patienten vor (Comella 2003 und 2007; Havlikova et al. 2011; Khedr et al. 2013; Lees et al. 1988; Poewe 2008; Svensson et al. 2012). Ihre Frequenz nimmt mit Fortschreiten der Erkrankung zu. Sie können grob eingeteilt werden in solche, die direkt durch die nächtliche motorische Symptomatik den Schlaf der Patienten beeinflussen, und solche, die aufgrund des neurodegenerativen Prozesses bestimmter Hirnregionen als primäre Manifestationen des idiopathischen Parkinson-Syndroms angesehen werden müssen, wie z.B. die REM-SchlafVerhaltensstörung (REM sleep behaviour disorder, RBD) (Comella 2007).

Nächtliches Erwachen und Schlaffragmentierung sind die häufigsten und am stärksten belastenden Störungen und führen zu einem rastlosen und nicht erholsamen Schlaf des Patienten. Die damit verbundene Reduktion der Gesamt-Schlafdauer führt zu vermehrter Tagesmüdigkeit und kann plötzliche Einschlafattacken im Tagesverlauf zur Folge haben (Comella 2007). Darüber hinaus berichten Patienten von Einschlafstörungen, nächtlicher Inkontinenz, schmerzhaften Dystonien und Krämpfen in den Extremitäten sowie nächtlichen Verwirrtheitszuständen und Halluzinationen (Khedr et al. 2013; Poewe 2008). Ursächlich werden die Schlaf-Wach-Störungen mit dem Auftreten motorischer Symptome mit nächtlicher Akinese, periodischen Beinbewegungen während des Schlafes (periodic limb movements in sleep, PLMS) und schlafbezogenen Atmungsstörungen in Zusammenhang gebracht (Comella 2003). Als weitere Ursachen werden der neurodegenerative Prozess selbst, Nebenwirkungen der dopaminergen Medikation sowie Komorbidität, insbesondere depressive Störungen und Nykturie, diskutiert (Comella 2007; Svensson et al. 2012).

Exzessive Tagesschläfrigkeit, die verschiedenen Untersuchungen zufolge von 15 bis 50 Prozent der Parkinson-Patienten angegeben wird (Comella 2007, Havlikova et al. 2011; Poewe 2008), ist mit einem schwerwiegenderen Verlauf des idiopathischen Parkinson-Syndroms, mit größeren krankheitsbedingten körperlichen Einschränkungen und einem früheren kognitiven Abbau der Patienten assoziiert (Comella 2007).

Eine REM-Schlaf-Verhaltensstörung wird als Syndrom mit ausgeprägter motorischer Aktivität während des REM-Schlafes bei Verlust der in diesen Schlafphasen physiologischen Muskelatonie beschrieben und kann zu einem mitunter gewaltsamen Ausagieren der Trauminhalte führen (Poewe 2008). Sie sind so bei einem Viertel bis der Hälfte aller Parkinson-Patienten Grund für nächtliche Störungen und einen unerholsamen Schlaf (Comella 2007; Sixel-Döring et al. 2011).

Zudem kommt es in etwa zwanzig Prozent der Fälle zu einer Koexistenz des idiopathischen Parkinson-Syndroms und des Restless-Legs-Syndroms (RLS) (Svensson et al. 2012), welches sich 
durch nächtliche Dysästhesien vorwiegend der unteren Extremitäten und einem dadurch hervorgerufenen Bewegungsdrang der Beine auszeichnet.

\subsection{Andere nicht-motorische Symptome}

Bei der Mehrheit der Parkinson-Patienten entstehen im Krankheitsverlauf autonome Dysfunktionen durch Fehlregulationen des vegetativen Nervensystems. Diese äußern sich bei $30-60 \%$ der Patienten in Blasenfunktionsstörungen wie zumeist einer Detrusorhyperaktivität mit imperativem Harndrang, erhöhter Miktionsfrequenz und Nykturie (Khedr et al. 2013; Poewe 2008; Schulz und Gasser 2007). Eine symptomatische orthostatische Hypotension kann in bis zu 30\% der Fälle, eine erektile Dysfunktion des Mannes in 15\% der Fälle in fortgeschritteneren Erkrankungsstadien spontan auftreten oder durch Medikamente induziert werden (Poewe 2008; Wüllner et al. 2007). Das frühe Auftreten dieser vegetativen Fehlregulationen spricht dabei eher gegen das Vorliegen eines idiopathischen Parkinson-Syndroms und für die Multisystematrophie.

Bei mehr als 2/3 der über 60-jährigen Patienten manifestiert sich eine Funktionsstörung des Intestinaltrakts in Form von Obstipation, die im Verlauf größtenteils medikamentös behandlungspflichtig wird (Del Tredici und Jost 2012; Khedr et al. 2013; Schulz und Gasser 2007). Daneben imponiert die gestörte Motilität des oberen Gastrointestinaltrakts in Form von Schluckstörungen, welche bei normaler Speichelproduktion oftmals eine störende Sialorrhö verursachen, sowie einer Magenentleerungsstörung. Die gestörte Magenmotilität führt neben unspezifischen Symptomen wie Völlegefühl und Sodbrennen auch zu einer verzögerten und reduzierten Medikamentenaufnahme und ist somit von erheblicher klinischer Relevanz (Del Tredici und Jost 2012). Darüber hinaus geben etwa 50\% der Patienten körperliche Schmerzen an, welche in der Mehrzahl der Fälle muskuloskeletaler Genese sind oder auf schmerzhafte dystone Bewegungen und Körperhaltungen zurückzuführen sind (Hanagasi et al. 2011; Poewe 2008). Darüber hinaus wird von schmerzhaften Sensationen berichtet, die sich ursächlich weder auf muskuloskeletale oder neuropathische noch auf andere altersbedingte körperliche Veränderungen zurückführen lassen. Pathophysiologisch geht man diesbezüglich von einer veränderten Schmerzwahrnehmung im zentralen Nervensystem aus (Poewe 2008).

Ein weiterer Ausdruck der Schädigung des autonomen Nervensystems ist das gehäufte Vorkommen einer Hyperhidrosis und einer Seborrhö, die das so genannte Salbengesicht vieler Parkinsonpatienten bedingt (Schulz und Gasser 2007).

\subsubsection{Pathophysiologie}

Das idiopathische Parkinson-Syndrom ist eine degenerative Stammganglienerkrankung, der pathophysiologisch ein Verlust überwiegend dopaminerger Neurone in umschriebenen Bereichen motorischer, limbischer und autonomer Kerngebiete zu Grunde liegt (Schulz und Gasser 2007). Die 
Atrophie melaninhaltiger Zellen in der Substantia nigra bedingt neben einem Verlust der Pigmentierung des Mittelhirns eine verminderte zerebrale Produktion des Neurotransmitters Dopamin. Der konsekutive Dopaminmangel führt zu einem Ungleichgewicht im Regelkreis exzitatorischer und inhibitorischer Neurone im Bewegungssystem. Darüber hinaus kommt es zu einem Zelluntergang in extranigralen dopaminergen und nicht-dopaminergen Nervenbahnen, die die bereits beschriebenen Funktionsbeeinträchtigungen im limbischen und vegetativen System zur Folge haben (Braak et al. 2000; Jellinger 2012).

$\mathrm{Zu}$ den neuropathologischen Veränderungen des idiopathischen Parkinson-Syndroms gehört das Vorkommen von intrazytoplasmatischen Einschlusskörperchen, den so genannten Lewy Bodies, welche vor allem aus fehlgeformtem $\alpha$-Synuklein bestehen. Diese führen zu einem vorzeitigen Zelluntergang der betroffenen Neurone und sind für die postmortale Bestätigung der klinischen Diagnose zwingend erforderlich (Braak et al. 2000; Jellinger 2012; Schulz und Gasser 2007).

Verschiedene Erklärungsmodelle bezüglich der Genese der frühzeitigen Neurodegeneration wurden in den letzten Jahren formuliert. Mit Ausnahme der erblichen Formen sieht das derzeit favorisierte Modell ein Zusammenwirken verschiedener Faktoren wie genetischer Prädisposition und Umwelteinflüssen ursächlich für den Zelluntergang (Houlden und Singleton 2012; Schulz und Gasser 2007). Auch sollen ein vermehrter oxidativer Stress und eine mitochondriale Fehlfunktion der betroffenen Neurone eine wichtige Rolle spielen (Ross et al. 2001; Schulz und Gasser 2007). Die Gültigkeit dieser Thesen ist jedoch noch nicht ausreichend belegt (Jellinger 2012).

\subsubsection{Diagnostik und Differentialdiagnosen}

Die Diagnose des idiopathischen Parkinson-Syndroms wird nach wie vor klinisch gestellt und orientiert sich an den in Tabelle 1 dargestellten Kriterien der UK Parkinson's Disease Society Brain Bank (Leitlinie Parkinson-Syndrome 2012; Litvan et al. 2003). 
Tabelle 1: Diagnosekriterien des idiopathischen Parkinson-Syndroms nach der UK Parkinson's Disease Society Brain Bank

\section{Schritt: Diagnose eines Parkinson Syndroms}

- Bradykinese (verlangsamte Initiation von Willkürbewegungen mit progressiver Reduktion der Geschwindigkeit und der Amplitude bei repetitiven Aktionen) und mindestens eines der folgenden Symptome:

- muskuläre Rigidität

- 4-6 Hz Ruhetremor

- posturale Instabilität, die nicht durch primär visuelle, vestibuläre, zerebelläre oder propriozeptive Dysfunktion verursacht wird

2. Schritt: Ausschlusskriterien für das idiopathische Parkinson-Syndrom

- Anamnese für wiederholte Schlaganfälle mit zunehmender Progression von Parkinson Symptomen

- Anamnese für wiederholte Schädel-Hirn-Traumata

- Anamnese für eine Enzephalitis

- okulogyre Krisen

- Neuroleptikagabe zu Beginn der Symptome

- mehr als ein betroffener Verwandter

- anhaltende Remission

- strikt unilaterale Symptome nach 3 Jahren

- supranukleäre Blickparese

- zerebelläre Zeichen

- frühe schwerwiegende autonome Störungen

- frühe schwere Demenz mit Beeinträchtigung von Gedächtnis, Sprache und Praxie

- positives Babinski Zeichen

- Vorliegen eines zerebralen Tumors oder eines kommunizierenden Hydrozephalus in der CCT

- negative Antwort auf hohe Levodopa-Dosen (wenn eine Malabsorption ausgeschlossen ist)

- eine vorherige Exposition mit MPTP (1-Methyl-4-Phenyl-1,2,3,6-Tetrahydropyridine)

3. Schritt: Supportive prospektive positive Kriterien für ein idiopathisches Parkinson-Syndrom (drei oder mehr Kriterien sind erforderlich für die definitive Diagnose eines idiopathischen ParkinsonSyndroms)

- unilateraler Beginn

- Ruhetremor

- progressive Erkrankung

- persistierende Asymmetrie, die die Seite des Beginns betrifft

- exzellente Antwort (70\%-100\%) auf Levodopa

- schwere Levodopa-induzierte Chorea/Dyskinesien

- Levodopa-Antwort für fünf oder mehr Jahre

- klinischer Verlauf von zehn oder mehr Jahren 
Differentialdiagnostisch ist das idiopathische Parkinson-Syndrom von weiteren Parkinson-Syndromen im Rahmen verschiedener anderer Systemerkrankungen abzugrenzen. Hierzu zählen neben der Multisystematrophie, die Kortikobasale Degeneration, die Progressive Supranukleäre Blickparese und die Lewy-Body-Demenz (Leitlinie Parkinson-Syndrome 2012). Die häufigste Form der symptomatischen Parkinson-Syndrome ist das medikamenten-induzierte Parkinson-Syndrom (Munhoz et al. 2010). Auslösende Medikamente sind hierbei typischerweise klassische Neuroleptika mit Dopaminrezeptor-blockierender Wirkung wie beispielsweise Haloperidol und Fluphenazin. Seltenere Ursachen sind Intoxikationen mit MPTP (1-Methyl-4-phenyl-1, 2, 3, 6-tetrahydropyridin), Kohlenmonoxid und Mangan sowie hypoxische und traumatische Hirnschäden und die virale Encephalitis lethargica. Darüber hinaus müssen differentialdiagnostisch der essentielle Tremor, der Normaldruck-Hydrozephalus und der Morbus Wilson in Betracht gezogen werden (Leitlinie Parkinson-Syndrome 2012; Schulz und Gasser 2007).

\subsubsection{Therapie}

Die Therapie des idiopathischen Parkinson-Syndroms sollte rechtzeitig und effizient beginnen und ein breites Spektrum medikamentöser, physiotherapeutischer, logopädischer und chirurgischer Maßnahmen umfassen. Sie erfolgt nach wie vor rein symptomatisch, da eine kausale Therapie aufgrund der bis heute noch ungeklärten Genese der Erkrankung nicht zur Verfügung steht.

Die pharmakologische Therapie zielt auf die Wiederherstellung des Gleichgewichts der Neurotransmitter ab und umfasst folgende Wirkstoffklassen: Levodopa in fester Kombination mit einem peripheren Decarboxylaseinhibitor, Dopaminrezeptoragonisten, MAO-B-Hemmer, COMTHemmer, NMDA-Rezeptor-Antagonisten und Anticholinergika. Die Wahl der medikamentösen Therapie richtet sich nach dem Alter des Patienten, Art und Schweregrad der klinischen Symptomatik sowie dessen Verlauf (Leitlinie Parkinson-Syndrome 2012; Schulz und Gasser 2007).

In der Frühphase der Erkrankung ist bei Patienten unter 70 Jahren eine Monotherapie mit Dopaminrezeptoragonisten indiziert, um die Einnahme von Levodopa hinauszuzögern, die bei frühem Einsatz mit einem erhöhten Risiko von Fluktuationen und Hyperkinesien im Krankheitsverlauf verbunden ist. Patienten über 70 Jahre werden initial mit Levodopa in fester Kombination mit einem peripheren Decarboxylaseinhibitor therapiert. Bei milder Symptomatik kann auch eine Monotherapie mit Rasagilin oder Selegilin erfolgen (Leitlinie Parkinson-Syndrome 2012). Im weiteren Krankheitsverlauf ist je nach Symptomkonstellation die kombinierte Gabe von Levodopa mit Dopaminrezeptoragonisten, COMT-Hemmern oder Amantadin sinnvoll (Leitlinie ParkinsonSyndrome 2012; Nutt und Wooten 2005). Zur Verringerung der on/off-Oszillationen in fortgeschrittenen Erkrankungsstadien kann eine subkutane Injektionstherapie mit Apomorphin oder eine intrajejunale L-Dopa-Infusionstherapie (Duodopa-Pumpe) erfolgen (Leitlinie ParkinsonSyndrome 2012). 
Bei Patienten mit dem Tremor-dominanten Typ des idiopathischen Parkinson-Syndroms kommen primär Budipin und Selegilin sowie Dopaminrezeptoragonisten zum Einsatz.

Grundsätzlich wird versucht, die Therapie im Rahmen der Empfehlungen der deutschen Gesellschaft für Neurologie so weit wie möglich den Erwartungen und dem individuellen Lebensplan des Patienten anzupassen (Leitlinie Parkinson-Syndrome 2012).

Bei Versagen der medikamentösen Therapie steht als Ultima Ratio die chirurgische TiefenhirnStimulation zur Verfügung. Mittels stereotaktisch eingebrachter Elektroden werden hierbei bestimmte subkortikale Kerngebiete (meist der Nucleus subthalamicus) von extern stimuliert, und es kommt so zu einer Reduktion der motorischen Kernsymptomatik (Leitlinie Parkinson-Syndrome 2012; Schulz und Gasser 2007).

Während des gesamten Krankheitsverlaufs profitieren die Patienten von einer physio- und ergotherapeutischen Mitbehandlung (Comella et al. 1994; Leitlinie Parkinson-Syndrome 2012). Mit Hilfe von gezielten Bewegungsübungen können die parkinsonsche Fehlhaltung korrigiert, verloren gegangene physiologische Bewegungsmuster durch bewusste Willkürbewegungen ersetzt und die allgemeine Bewegungsarmut verbessert werden. Massagen und Bewegungsbäder helfen zusätzlich, muskuläre Verspannungen zu lindern. Patienten mit Dysarthrophonie ist zudem zu einer logopädischen Behandlung zu raten, um einer drohenden sozialen Isolierung durch eine zunehmende Artikulationsstörungen und der damit verbundenen Unfähigkeit, sich in gesellschaftlichem Rahmen verständlich zu machen, entgegen zu wirken (Leitlinie Parkinson-Syndrome 2012; Schulz und Gasser 2007).

\subsubsection{Verlauf und Komplikationen}

Der Krankheitsverlauf des idiopathischen Parkinson-Syndroms ist individuell sehr unterschiedlich. Allgemein sind der Tremordominanz- und der Äquivalenztyp prognostisch günstiger als der Verlauf bei vorherrschender akinetisch-rigider Symptomatik (Schulz und Gasser 2007). Die Erkrankung ist chronisch progredient und führt so im Verlauf zu einem allmählichen Fortschreiten der Funktionseinschränkungen. Sie ist durch akute, teilweise starke Fluktuationen der Beweglichkeit gekennzeichnet, die mit Erkrankungs- und Therapiedauer zunehmen. Die antiparkinsonschen Effekte der Therapie mit L-Dopa lassen nach etwa zwei bis fünf Jahren nach (wearing-off). Die Verkürzung der Wirkdauer führt dann in Abhängigkeit von der Einnahmezeit der Medikamente regelmäßig zu den so genannten end-of-dose-Akinesien. Im weiteren Verlauf kommt es zusätzlich zu dosisunabhängigen off-Phasen, die durch eine nahezu völlige Bewegungsunfähigkeit des Patienten gekennzeichnet sind. Der Übergang zu den on-Phasen mit guter Beweglichkeit geschieht abrupt und kann ebenso wenig wie das Auftreten der off-Phasen willentlich vom Patienten beeinflusst werden. Mit zunehmender Therapiedauer zeichnen sich die on-Phasen zudem vermehrt durch dystone Bewegungstörungen und allgemeine Hyperkinesien aus, die trotz der damit einhergehenden Verschlechterung der 
Beweglichkeit nicht selten eine Reduktion der L-Dopa-Dosis erzwingen (Leitlinie ParkinsonSyndrome 2012; Schulz und Gasser 2007).

Die pharmakologische Therapie kann einige weitere ungewollte Wirkungen hervorrufen, die den Verlauf der Parkinsonerkrankung verkomplizieren können. Alle Anti-Parkinson-Medikamente können die Entwicklung psychotischer Symptome induzieren, sodass etwa zehn bis 30\% der Patienten mit einem Parkinson-Syndrom während der Langzeittherapie illusionäre Verkennungen, visuelle Halluzinationen, Verwirrtheitszustände oder seltener paranoide Störungen entwickeln (Leitlinie Parkinson-Syndrome 2012). Dopaminagonisten verstärken zudem die durch die Krankheit an sich bestehende Tagesschläfrigkeit in unterschiedlichem Grad. In Fällen, in denen die Verkürzung der Einschlaflatenz besonders ausgeprägt ist und nicht durch einen Wechsel des verursachenden Medikaments behoben werden kann, muss von einer Untauglichkeit des Patienten zum Führen eines Kraftfahrzeugs ausgegangen werden (Leitlinie Parkinson-Syndrome 2012). Darüber hinaus kann die Einnahme von Dopaminagonisten das Risiko einer orthostatischen Dysregulation erhöhen. Die latente Sturzgefahr, die aufgrund der posturalen Instabilität und des pathologischen Gangbildes des Patienten ohnehin besteht, wird so zusätzlich verstärkt.

Eine weitere schwere Komplikation im Verlauf der Erkrankung ist das Auftreten einer akinetischen Krise. Durch Infekte, Operationen, abrupte Therapieabbrïche oder die Verabreichung bestimmter Medikamente (z.B. klassische Neuroleptika) kann eine bis zu Tage anhaltende Bewegungsblockade mit ausgeprägtem Rigor, Hyperthermie und Hyperhidrosis verursacht werden. Besonders in späten Erkrankungsstadien stellt dies aufgrund der Gefahr einer Aspirationspneumonie und Exsikkose einen lebensbedrohlichen Zustand dar (Schulz und Gasser 2007).

Die Lebenserwartung von Patienten mit idiopathischem Parkinson-Syndrom ist allgemein etwas herabgesetzt. Im Vergleich mit einer altersentsprechenden Referenzgruppe besteht für die ParkinsonPatienten ein 2-fach erhöhtes Risiko zu sterben (D'Amelio et al. 2006; Schulz und Gasser 2007). Die Sterblichkeit korreliert hierbei streng mit der Erkrankungsdauer. In den ersten fünf Erkrankungsjahren liegt das relative Risiko bei 1.1, nach fünf bis zehn Jahren bei 2.3 und nach über zehn Jahren bei 3.5 (Chen et al. 2006). Bezüglich der Todesursachen besteht ein signifikanter Unterschied für Pneumonie und Kachexie zwischen Parkinson-Patienten und der altersentsprechenden Referenzgruppe ohne neurologische Erkrankungen (D’Amelio et al. 2006; Schulz und Gasser 2007).

\subsubsection{Lebensqualität}

Das idiopathische Parkinsonsyndrom ist eine chronisch progrediente Erkrankung, die die betroffenen Patienten nicht nur in ihrer Mobilität beeinträchtigt, sondern sich auch belastend auf zahlreiche weitere Bereiche des täglichen Lebens auswirkt. Mit den krankheitsbedingten Einschränkungen der 
körperlichen Leistungsfähigkeit und seelischen Integrität geht zumeist eine schwere Beeinträchtigung der Lebensqualität der Patienten einher.

Die Reduktion der Lebensqualität der Patienten hängt u.a. mit einer Beeinträchtigung der Mobilität durch eine verminderte körperliche Funktionsfähigkeit zusammen (Schrag et al. 2000). Je fortgeschrittener dabei das Erkrankungsstadium, desto schwerwiegender sind die Lebensqualitätsbereiche „körperliche Funktionsfähigkeit“, „körperliche Rollenfunktion“, „Vitalität“ und „allgemeine Gesundheit“" der betroffenen Patienten eingeschränkt (Kuopio et al. 2000).

Darüber hinaus tragen nach neueren Erkenntnissen die nicht-motorischen Symptome des iPS erheblich zu der Beeinträchtigung der gesundheitsbezogenen Lebensqualität der Patienten bei (Antonini et al. 2012; Bosboom et al. 2004; Khedr et al. 2013) und haben sich zu einem hauptsächlichen Prognosefaktor für die allgemeine Belastung durch die Erkrankung und die Alltagsfähigkeit der Patienten entwickelt (Poewe 2008). Die Lebensqualität der Patienten hängt zwar von individuell sehr unterschiedlichen Faktoren ab, wird im Allgemeinen jedoch eher von psychosozialen als von körperlichen Determinanten bestimmt (Lee et al. 2006) und kann sich schon innerhalb einer relativ kurzen Zeit (2 Jahre) deutlich verschlechtern (Visser et al. 2009). Ein enger Zusammenhang wurde wiederholt zwischen einer vergleichsweise verminderten Lebensqualität und dem Vorhandensein depressiver Symptome festgestellt (Antonini et al. 2012; Kuopio et al. 2000; Lee et al. 2006; Quin et al. 2009). Patienten mit vegetativen Dysfunktionen, nächtlichen Schlafstörungen und einem beginnenden kognitiven Abbau haben zudem ein besonders erhöhtes Risiko für eine Verschlechterung der gesundheitsbezogenen Lebensqualität. Diese Beeinträchtigung der Lebensqualität ist wiederum mit einer Zunahme an psychosozialen Problemen, depressiven Symptomen und kognitiver Dysfunktion assoziiert (Visser et al. 2009).

Das gehäufte Auftreten nächtlicher Schlafstörungen und die oftmals resultierende Tagesmüdigkeit wirken sich ebenfalls negativ auf die Lebensqualität der betroffenen Patienten aus und sind stark mit dem Vorhandensein depressiver Symptome verbunden (Happe und Berger 2003; Havlikova et al. 2011; Quin et al. 2009).

Ein weiterer wichtiger Faktor ist die durch die Erkrankung hervorgerufene allgemeine Erschöpfung der Patienten (fatigue) (Antonini et al. 2012; Havlikova et al. 2008; Herlofson und Larsen 2003). Diese Erschöpfung ist durch eine extreme körperliche und mentale Müdigkeit und Schwäche sowie eine Armut an Energie und Initiative der Patienten gekennzeichnet. Das Auftreten einer krankheitsbedingten allgemeinen Erschöpfung beeinträchtigt alle Bereiche der gesundheitsbezogenen Lebensqualität der Patienten, besonders die Bereiche „körperliche Beschwerden“, „Mobilität“ und „emotionales Wohlbefinden“ (Havlikova et al. 2008).

Über diese die Lebensqualität der Parkinsonpatienten negativ beeinflussenden Faktoren hinaus, die auf die motorische und nicht-motorische Symptomatik der Erkrankung zurückzuführen sind, stellt auch die Tatsache, an einer unaufhaltsam fortschreitenden Erkrankung zu leiden, eine große seelische 
Belastung für die betroffenen Patienten dar. Jede im Krankheitsverlauf auftretende Komplikation, jede ungewünschte Wirkung der pharmakologischen Therapie und jeder erneute Krankenhausaufenthalt führen zu einer weiteren Beeinträchtigung ihrer Gesundheitsbezogenen Lebensqualität.

In der Bewältigung der Erkrankung spielen zudem so individuelle Faktoren wie Persönlichkeit, Lebenseinstellung, Grad der inneren Zufriedenheit und persönliche „Coping“-Strategien der Patienten eine wichtige Rolle. Ein guter familiärer Rückhalt, die Unterstützung durch ein soziales Netzwerk und der Zugang zu Gesundheitsresourcen erleichtern den Umgang mit der Erkrankung und beeinflussen ebenso die Lebensqualität der Patienten (Schrag 2001).

\subsection{Belastungserleben durch Angehörigenbetreuung}

\subsubsection{Familienpflege}

Im 20. Jahrhundert unterlag die Bevölkerung in der westlichen Gesellschaft stärkeren demographischen, kulturellen und strukturellen Veränderungen als jemals zuvor. Die gestiegene durchschnittliche Lebenserwartung sowie die besonders in den letzten Jahrzehnten gesunkenen Geburtenraten haben zu einem demographischen Wandel weg von der Bevölkerungspyramide hin zu einer Bevölkerungssäule beziehungsweise zu einem Bevölkerungspilz geführt (Bengtson und Martin 2001). Die Familienstrukturen haben sich so von einer zuvor eher vertikalen zu einer nun eher horizontalen Einheit entwickelt mit einer größeren Anzahl an miteinander lebenden Generationen aber mit einer geringeren Anzahl an Mitgliedern in jeder dieser Generationen (Lowenstein 1999). Wo sich bislang einige wenige lebende Großeltern auf eine vergleichsweise größere Anzahl an Kindern und eine noch größere Anzahl an Enkelkindern verteilten, kommt nun eine steigende Zahl an Groß- und Urgroßeltern auf immer weniger Kinder und Enkelkinder (Bengtson und Martin 2001; Brody 1981; vgl. Linkersdörfer 2006).

Sicherlich darf das fortgeschrittene Lebensalter nicht per se mit Krankheit und Hilfsbedürftigkeit gleichgesetzt werden, doch besteht zwischen diesen beiden Variablen ein offensichtlicher Zusammenhang. So nimmt der Anteil der pflegebedürftigen Personen in der Bundesrepublik Deutschland von 2\% im Alter zwischen 65 bis 69 Jahren auf 28\% in der Gruppe der über 85 -jährigen zu (Brody 1981; Tesch-Römer 2001). Neben einer Zunahme dieser älteren Bevölkerungsanteile bringt das allgemeine Altern der Gesellschaft auch ein durchschnittlich höheres Alter der potentiell für die Pflegeübernahme zur Verfügung stehenden Personen mit sich, die mitunter selber schon aufgrund ihres vorangeschrittenen Alters in ihrer körperlichen und geistigen Leistungsfähigkeit eingeschränkt sein können (Bengtson und Martin 2001; Brody 1981; Lowenstein 1999).

Gleichzeitig führen steigende Scheidungsraten, die sich in Europa seit den 1970er Jahren verdoppelt haben, und vermehrte Wiederheirat zu einem häufig komplexen Familiengeflecht aus biologischen Kindern und Enkeln, Stiefkindern und Stiefenkeln sowie Schwiegerkindern und Ex-Schwiegerkindern, 
in dem Erwachsene zum ersten Mal mehr Eltern als Kinder haben können (Bengtson und Martin 2001; Lowenstein 1999). Diese neue Familienstruktur kann einerseits eine Diskontinuität der Eltern-KindBeziehung zum getrennt lebenden Elternteil und somit eine Verminderung des Verpflichtungsgefühls diesem Elternteil gegenüber bedingen. Andererseits kann die familiäre Situation für die erwachsenen Kinder aber auch eine doppelte Verpflichtung zu den in getrennten Haushalten lebenden leiblichen Eltern sowie eventuell zu deren neuen Lebenspartnern bedeuten (Schütze 1995, Schütze und Wagner 1991; vgl. Linkersdörfer 2006).

Ein weiterer wichtiger Punkt in Hinblick auf die sozialen und kulturellen Veränderungen in der westlichen Gesellschaft ist die gestiegene Erwerbstätigkeit der Frauen. Waren es bislang mit überwältigender Mehrheit Frauen, die traditionell die Pflege hilfsbedürftiger Familienmitglieder übernahmen, so hat mit steigender fachlicher Qualifikation die Bereitschaft der Frauen, zugunsten der Familie im Beruf zurückzustecken beziehungsweise diesen ganz aufzugeben, in den letzten Jahren deutlich abgenommen (Bengtson und Martin 2001; Brody 1981; Tesch-Römer 2001). Darüber hinaus wird der Pool der zur Pflege zur Verfügung stehenden Familienmitglieder noch weiter durch die allgemein erhöhte berufliche Mobilität und die größere geographische Entfernung zwischen Eltern und ihren erwachsenen Kindern reduziert (Bengtson und Martin 2001; Lowenstein 1999).

Die Motive für die Übernahme der Pflege eines Familienangehörigen sind vielfältig: traditionelle Familienwerte, Pflichtgefühl, Notwendigkeit, finanzielle Aspekte, moralische Normen und gesellschaftliche Zwänge sowie Gefühle der Zuneigung und Verbundenheit mit dem Pflegebedürftigen und auch altruistische Gründe (Funk 2010; Jones et al. 2011; Lowenstein 1999; Silverstein et al. 2002; Tesch-Römer 2001). Hierbei ist die letztendliche Entscheidung jedes einzelnen zur Übernahme der Pflegerolle zumeist auf ein komplexes Zusammenspiel verschiedener Beweggründe zurück zu führen. Sie beeinflussen dabei nicht nur die Motivation zur Übernahme der Pflege sondern auch Entscheidungen und Verhalten innerhalb der Pflegebeziehung (Funk und Kobayashi 2009). Ebenso verschieden wie die Motive sind auch die Formen der geleisteten Pflege. Je nach Hintergrund und persönlichem Anspruch kann sich die Pflege eines Familienmitglieds auf die rein emotionale Unterstützung beschränken oder aber auch instrumentelle - sprich körperliche - Pflege beinhalten. Zudem kann die Betreuung sowohl einen informativen als auch finanziellen Charakter haben (Edwards und Ruettiger 2002; Tesch-Römer 2001). Die Pflege ist dabei keine statische Situation sondern ein stufenweiser Prozess, der sich im Laufe der Zeit allmählich entwickelt und wandelt (Cicirelli 2000; Dyck 2009; Tesch-Römer 2001). Laut Cicirelli (2000) wird das erste Stadium in der Pflegebeziehung zu einem alternden Elternteil zumeist durch beobachtendes Verhalten von Seiten des erwachsenen Kindes geprägt. Der gute Gesundheitszustand der Eltern wird nicht länger als gegeben und unveränderlich hingenommen, sondern das Kind beginnt, ein „Auge“ auf die Eltern zu haben, ohne dabei seine diesbezüglichen Sorgen und Ängste zu kommunizieren (emotionale Pflege). Dem schließt sich ein Stadium des Ratgebens an, in dem das Kind die Eltern bittet und ermahnt, sich mit 
bestehenden Symptomen auseinander $\mathrm{zu}$ setzen und ein bestimmtes Gesundheitsverhalten einzunehmen (informative Pflege). Bei ausreichender Ernsthaftigkeit der elterlichen Symptome beginnt die dritte Stufe, die sich durch die tatsächliche - auch körperliche - Unterstützung des nun hilfsbedürftigen Elternteils auszeichnet (instrumentelle Pflege) (Cicirelli 2000).

Die Beziehungen und Interaktionen zwischen den verschiedenen Generationen innerhalb einer Familie können unter dem Begriff ,intergenerationelle Solidarität“ zusammengefasst werden. Besondere Merkmale dieser Familiensolidarität sind die Bi-Direktionalität und die Reziprozität der Beziehung (Lowenstein 1999; Marcoen 2005; vgl. Silverstein et al. 2002). Der gegenseitige Austausch sowohl instrumenteller als auch materieller und emotionaler Unterstützung fließt in beide Richtungen, wobei die Gegenseitigkeit entweder unmittelbar oder im Verlauf der Beziehung über Jahre ersichtlich wird (Lowenstein 1999; vgl. Daatland und Lowenstein 2005; vgl. Schwarz und Trommsdorff 2005). Für jede Generationen ist das Geben generell nicht weniger wichtig als zu Nehmen, da beides Auswirkungen auf die Interaktionen und das Wohlergehen der beteiligten Personen hat. Die Anteile der einzelnen Familienmitglieder sind hierbei allerdings selten gleich groß (Lowenstein 1999).

Im Zusammenhang mit intergenerationeller Solidarität sollte auch die intergenerationelle Ambivalenz nicht unerwähnt bleiben. Konflikte zwischen Normen und Rollen der verschiedenen Generationen führen sowohl auf öffentlich sozialer als auch auf zwischenmenschlich privater Ebene zu Widersprüchen in der erwachsenen Eltern-Kind-Beziehung (Lowenstein 1999). Diese Gegensätzlichkeiten mit zugleich positiven und negativen Gefühlen resultieren in intergenerationeller Ambivalenz, die durch die Situation der Familienpflege noch weiter verstärkt wird. Der erfolgreiche Umgang mit dieser Ambivalenz ist jedoch wichtig, um die intergenerationelle Solidarität innerhalb einer Familie zu erhalten (Tesch-Römer 2001).

\subsubsection{Belastungserleben durch die Pflegesituation}

\subsubsection{Belastung in der Pflege}

Die Pflege von Familienangehörigen, die altersbedingt oder aufgrund einer chronischen Krankheit hilfsbedürftig sind, ist seit mehr als 30 Jahren als chronischer Stressfaktor bekannt (Perrig-Chiello und Sturzenegger 2001; Vitaliano et al. 2004). Eine besondere Herausforderung für erwachsene Kinder stellt dabei die Übernahme der Pflegerolle für die eigenen alternden Eltern dar. Sie sehen sich einerseits mit dem Problem konfrontiert, eine Balance zwischen den elterlichen Bedürfnissen und den eigenen ökonomischen, räumlichen und psychischen Voraussetzungen zu finden. Andererseits müssen sie versuchen, sowohl die eigene als auch die elterliche Autonomie zu bewahren, aber gleichzeitig ihrer Verpflichtung zur Verantwortung für die alternden Eltern nachzukommen (Schütze und Wagner 1991). Aus Sicht der erwachsenen Kinder wird die Entscheidung zur Pflegeübernahme häufig von ambivalenten Gefühlen begleitet, die durch die Diskrepanz zwischen den empfundenen hohen 
elterlichen Erwartungen und Wünschen und dem eigenen Streben nach Unabhängigkeit hervorgerufen werden (Funk und Kobayashi 2009; Perrig-Chiello und Sturzenegger 2001). Der teilweise enorme Zeitaufwand sowie die womöglich jahrelange Dauer der Pflege bringen weitreichende Konsequenzen mit sich und können so zu einer großen Belastung im Leben der Pflegenden führen. Belastung wird hierbei im Allgemeinen als das Ausmaß definiert, in dem der Pflegende seine Gesundheit, sein Sozialleben und seine finanzielle Situation durch die Pflege negativ beeinflusst sieht (Edwards und Ruettiger 2002). Die Pflegenden sehen sich im Verlauf der Pflege zunehmend einer sozialen Isolation ausgesetzt, da die Pflege die zur Verfügung stehende freie Zeit der Pflegenden mitunter massiv einschränkt und sich somit negativ auf deren soziale Kontakte auswirkt (Adler et al. 1996; McLaughlin et al. 2010; Schrag et al. 2006; Vitaliano et al. 2004). Die eigene Zeitplanung wird unflexibel, spontane Ausflüge oder Urlaube können nicht oder nur schwer realisiert werden, und eine Anpassung beziehungsweise Unterordnung der eigenen Zukunftspläne unter die Pflege erscheint notwendig. Dies führt dazu, dass die Pflegenden sich oftmals durch die Pflege angebunden fühlen und einen Verlust über die Kontrolle der eigenen Lebensereignisse beklagen. Die Unvorhersehbarkeit des Krankheitsverlaufs des pflegebedürftigen Familienmitglieds kann dieses Gefühl zudem noch weiter verstärken (Adler et al. 1996; Brody 1985; Dyck 2009; Edwards und Ruettiger 2002; Thommessen et al. 2002). Darüber hinaus kommt es zu einer Doppelbelastung der Pflegenden durch die familiären Verpflichtungen in Haushalt und Pflege einerseits und die beruflichen Anforderungen andererseits (Brody 1985; Edwards und Ruettiger 2002; Perrig-Chiello und Höpflinger 2005; Vitaliano et al. 2004). Bei dauerhafter Unvereinbarkeit der konkurrierenden Verpflichtungen beziehungsweise bei Überschreitung der diesbezüglichen individuellen Belastungsgrenze ist häufig der Rückzug der Pflegenden aus der Erwerbstätigkeit die Folge, was wiederum finanzielle Einbußen für die betroffenen Familien nach sich zieht. Die Familien der Pflegenden sind zudem insofern durch die Pflege belastet, als dass die durch die Pflege direkt beanspruchte Zeit nun in den Beziehungen der pflegenden Person zu ihrem Partner, den eigenen Kindern und anderen Familienmitgliedern fehlt (Brody 1985). Neben diesen Rollenkonflikten kann die Pflege - insbesondere in einer Pflegebeziehung zwischen alterndem Elternteil und erwachsenem Kind - zur Reaktivierung ungelöster Abhängigkeitskonflikte und längst überwunden geglaubten Aggressionen und alten Beziehungsproblemen innerhalb der Familie führen. Dadurch können Spannungen in der Pflegebeziehung selber, in den Partnerschaften der pflegenden Personen und in den Geschwisterbeziehungen entstehen (England und Tripp-Reimer 2003; Schütze und Wagner 1991).

Im Umgang mit pflegenden Angehörigen ist es zudem wichtig, zwischen objektiver Belastung und subjektivem Belastungserleben zu unterscheiden. So kann es durchaus vorkommen, dass Pflegende, die objektiv einer eher geringen Belastung ausgesetzt sind, unter dieser von ihnen selber als immens empfundenen Last zusammen brechen. Ebenso können Pflegende ihre Lebensqualität als sehr hoch einschätzen, obwohl sie objektiv gesehen unter einer vergleichsweise hohen Pflegebelastung stehen (Chappell und Reid 2002; Dyck 2009; Thommessen et al. 2002). 


\subsubsection{Auswirkungen der Belastung}

Die Pflegebelastung hat vielfältige Auswirkungen nicht nur auf die psychische und physische Gesundheit der Pflegenden sondern in ihrer Konsequenz auch auf die Pflege und somit wiederum auf die Pflegebedürftigen selbst (Chappell und Reid 2002; Lökk 2008; vgl. Linkersdörfer 2006).

Pflegende Angehörige haben verschiedenen Studien zufolge ein deutlich erhöhtes Risiko zur Entwicklung depressiver Symptome beziehungsweise einer manifesten, klinisch relevanten Depression (Årsland et al. 1999; Happe und Berger 2002; Lökk 2008; Martínez-Martín et al. 2007; Schrag et al. 2006; Vitaliano et al. 2004). Sie leiden im Vergleich zu nicht-pflegenden Personen unter einer geringeren Selbstachtung sowie einem geminderten Kompetenzempfinden und beklagen häufiger Hoffnungslosigkeit, Frustration und emotionale Überlastung (Brody 1985; Carter et al. 1998; Chappell und Reid 2002). Zudem geben sie vermehrt Angstsymptome, Reizbarkeit und Traurigkeit an und sind insgesamt unzufriedener mit ihren Lebensumständen (Årsland et al. 1999; Phillips et al. 2009). Darüber hinaus spielen Schlafstörungen und eine allgemeine lähmende Müdigkeit ein große Rolle im Belastungserleben pflegender Angehöriger (Cupidi et al. 2012; Happe und Berger 2002; Lökk 2008; Phillips et al. 2009; Thommessen et al. 2002) und führen zu einer vermehrten Einnahme von Schlafund Beruhigungsmitteln (Adler et al. 1996).

$\mathrm{Zu}$ den Auswirkungen der Belastung auf die körperliche Gesundheit der Pflegenden gehören neben Erkrankungen des Herz-Kreislauf-Systems, des Stoffwechsels und des Bewegungsapparates auch Muskelverspannungen sowie unspezifische Schmerz- und Erschöpfungszustände (Adler et al. 1996; Chappell und Reid 2002; Lökk 2008; Vitaliano et al. 2004). Eine vergleichsweise erhöhte Mortalität der pflegenden Angehörigen konnte ebenfalls nachgewiesen werden (O'Rourke et al. 2003; Vitaliano et al. 2004). Zudem konnte eine deutliche Beziehung zwischen dem pflegebedingt erhöhten mentalen Stress und physiologischen Veränderungen festgestellt werden. So wurden in Blutproben von pflegenden Angehörigen erhöhte Konzentrationen des Stresshormons Cortisol und konsekutiv gehäuft Hyperglykämien, Hyperinsulinämien sowie hypertensive Blutdruckwerte gemessen (Vitaliano et al. 2004). Auch scheint eine Verbindung zwischen dem Belastungserleben und den Immunfunktionen der Pflegenden zu bestehen (verminderte Tumorabwehr, reduzierte Antikörperfunktion und -produktion, verlangsamte Wundheilung). Unterschiede zwischen pflegenden und nicht-pflegenden Angehörigen wurden ebenso bezüglich Anzahl und Häufigkeit der eingenommenen Medikamente gegen somatische Beschwerden gefunden (Adler et al. 1996; Vitaliano et al. 2004).

Werden die Grenzen der Belastbarkeit der pflegenden Angehörigen im Verlauf einer jahrelangen Pflegebeziehung erreicht bzw. überschritten, wirkt sich dies nicht nur - wie bereits beschrieben - auf die Gesundheit der Pflegenden sondern auch auf die Qualität der Pflege aus (vgl. Chappell und Reid 2002). Der Ton wird rauer, der Umgang angestrengter, die Zeit für extra „Bonbons“ knapper. Die Pflege wird auf die Befriedigung der körperlichen Grundbedürfnisse der Gepflegten zurück gefahren. Sind die pflegenden Angehörigen nicht mehr in der Lage, ihren zunehmenden gesundheitlichen 
Einschränkungen mit suffizienten Strategien zur Stressbewältigung zu begegnen, kommt es vermehrt zur Inanspruchnahme von Pflegedienstleistungen und in letzter Konsequenz oft zur Heimunterbringung der Pflegebedürftigen (Chappell und Reid 2002). Dies birgt eine erhöhte Gefahr für Schuldgefühle. Auch kann dysfunktionales Coping der Betroffenen ohne Hilfestellungen von außen zu chronischem Stress, Alkoholabusus und körperlicher Gewalt gegenüber den Pflegebedürftigen führen (England und Tripp-Reimer 2003).

\subsubsection{Prädiktoren für Belastung}

Die Prädiktoren für das Belastungserleben sind mannigfaltig und hängen sowohl von den pflegenden Personen selber als auch von deren hilfsbedürftigen Angehörigen und den Umständen der Pflege ab (Vitaliano et al. 2004; vgl. Linkersdörfer 2006). Auf Seiten der Pflegenden spielen die allgemeine körperliche und seelische Gesundheit, das persönliche Ausmaß an Selbstvertrauen, Lebensfreude, optimistischer Lebenseinstellung und Stressempfinden, sowie die Qualität der emotionalen Beziehung zum Gepflegten eine bedeutende Rolle für das subjektive Belastungserleben der Betroffenen (Adler et al. 1996; Chappell und Reid 2002; England und Tripp-Reimer 2003; Karantzas et a. 2010; Shim et al. 2011). Weibliche Pflegende fühlen sich durch die Pflege allgemein stärker belastet als Männer und leiden so häufiger unter depressiven Symptomen und mit der Pflege assoziierten Schuldgefühlen (Hooker et al. 2000; Lökk 2008; Martínez-Martín et al. 2007; Yee und Schulz 2000). Auch beeinflusst der Verlust an Gemeinsamkeit und Gegenseitigkeit in der Beziehung zum gepflegten Angehörigen ihr Belastungserleben vergleichsweise negativer (Carter et al. 1998; Hooker et al. 2000; Shim et al. 2011). Zudem korreliert die empfundene Belastung mit der Zufriedenheit der Pflegenden über die eigene eheliche und sexuelle Beziehung (Schrag et al. 2006).

Auf Seiten der Gepflegten sind verschiedene Variabeln Prädiktoren für das Belastungserleben der betreuenden Angehörigen. Psychische Symptome wie allgemeine kognitive Defizite, Depression, Verwirrung und Wahnvorstellungen sowie Verhaltensauffälligkeiten wie Beschimpfungen, körperliche Aggressionen und destruktives Störverhalten spielen diesbezüglich eine wichtige Rolle (Årsland et al. 1999; Chappell und Reid 2002; England und Tripp-Reimer 2003; Lökk 2008; Shin et al. 2012). Funktionelle Defizite und eine zunehmend reduzierte Alltagsfähigkeit der Patienten tragen zum Ausmaß der körperlichen Pflegbedürftigkeit bei und sind ebenfalls von großer Bedeutung für die subjektive Belastung der Pflegenden (Martínez-Martín et al. 2007; Schrag et al. 2006; Thommessen et al. 2002; vgl. Linkersdörfer 2006). Insbesondere in Pflegebeziehungen mit Parkinsonpatienten bestehen enge Korrelationen zwischen dem Belastungserleben der Pflegenden und der Schwere der motorischen Dysfunktionen und diesbezüglichen Komplikationen, dem Auftreten von Halluzinationen, der Bradyphrenie sowie der Schlafstörungen der Patienten (Årsland et al. 1999; Caap-Ahlgren und Dehlin 2002; Happe und Berger 2002; Lökk 2008; Shin et al. 2012). Zudem scheinen das Vorhandensein einer reduzierten Lebensqualität und einer Depression des Patienten eng 
mit dem Auftreten depressiver Symptome beim betreuenden Angehörigen verbunden zu sein (Happe und Berger 2002).

Darüber hinaus beeinflussen die Umstände der Pflegesituation das Belastungserleben und die Anfälligkeit der Pflegenden für physische und psychische Erkrankungen (vgl. Linkersdörfer 2006). Ein verhältnismäßig hoher Pflegeeinsatz, eine lange Dauer der Pflegebeziehung, eine ungünstige Pflegegeschichte und das Zusammenleben mit der zu pflegenden Person gehen mit einer erhöhten Belastung einher (Adler et al. 1996; Chappell und Reid 2002; Happe und Berger 2002; Lökk 2008; Razali et al. 2010; Schrag et al. 2004; Vitaliano et al. 2004). Wichtige belastende Faktoren sind zudem die soziale Isolation durch die Pflege, eine geringe soziale Unterstützung der Pflegenden sowie familiäre Konflikte und Kommunikationsprobleme (Carter et al. 1998; Chappell und Reid 2002; McLaughlin et al. 2010; Shin et al. 2012; Vitaliano et al. 2004). Besonders in Geschwisterbeziehungen können aufgrund der elterlichen Pflege Spannungen entstehen. Mangelnde Unterstützung durch die Geschwister, Vertrauensbrüche und Streitigkeiten um ungeklärte Erbangelegenheiten spielen hierbei eine wichtige Rolle. Auch eine etwaige Diskrepanz zwischen dem jetzigen Pflegeeinsatz und der empfundenen früheren Favoritenstellung eines bestimmten Geschwisterteils bei den Eltern kann zu einem erhöhten subjektiven Belastungserleben der pflegenden Person führen (England und TrippReimer 2003).

$\mathrm{Zu}$ den zahlreichen Faktoren, die das Belastungserleben der Pflegenden reduzieren beziehungsweise sich positiv auf die Gesundheit der Pflegenden auswirken, zählen unter anderem ein starkes soziales Netzwerk aus familiären und extra-familiären Kontakten, durch die die Pflegenden soziale und emotionale Unterstützung erfahren, die freiwillige Entscheidung zur Pflegeübernahme sowie eine als hoch empfundene Familiensolidarität und eine offene und ehrliche Kommunikation sowohl innerhalb der eigenen Familien als auch innerhalb der Ursprungsfamilien der Pflegenden (Chappell und Reid 2002; Cicirelli 1993; Edwards und Ruettiger 2002; Perrig-Chiello und Sturzenegger 2001; vgl. Linkersdörfer 2006). Zudem scheinen eine angemessene Frequenz und Dauer von Ruhepausen und eine Inanspruchnahme von professionellen Pflegedienstleistungen wie beispielsweise Tagespflegeangebote, aber auch die Teilnahme an Gesprächs- und Selbsthilfegruppen zu einer Reduktion der Pflegebelastung zu führen (Andrén und Elmståhl 2008; Chappell und Reid 2002; Dyck 2009; Lowenstein 1999; Yee und Schulz 2000).

Darüber hinaus spielt die Qualität der Beziehung und das Bindungsverhalten des pflegenden Kindes an das zu pflegende Elternteil eine maßgebliche Rolle in der Belastungsverarbeitung (Adler et al. 1996; Karantzas et al. 2010; vgl. Linkersdörfer 2006). Starke, positive Bindungsstile sowie ein hohes $\mathrm{Maß}$ an in der frühen Eltern-Kind-Beziehung erlebten emotionalen Wärme, Empathie und Nähe scheinen sich protektiv auf das Belastungserleben auszuwirken (Cicirelli 1993; Daire 2002; Markiewicz et al. 1997). Unsicher gebundene pflegende Kinder erleben dabei eine insgesamt stärker empfundene allgemeine Belastung durch die Pflege, mehr negative Gefühle und damit auch stärkere 
Schwierigkeiten beim Ausfüllen der Pflegerolle als sicher gebundene erwachsene Kinder. Auch leiden Angehörige mit unsicherem Bindungsverhalten häufiger unter psychiatrischen Symptomen wie Depressionen und Angst (Carpenter 2001; Crispi et al. 1997; Magai und Cohen 1998). Während ängstlich-ambivalente Bindungen vermehrt mit negativen emotionalen Auswirkungen durch die Pflege und einem weniger ausgeprägten sozialen Netzwerk assoziiert sind (Karantzas et al. 2010; Markiewicz et al. 1997), berichten emotional abhängige Kinder von „stärker ausgeprägten Gefühlen von Ärger und Überforderung in der Versorgung" der Eltern (Adler et al. 1996, S. 148). Die Rate der Heimunterbringungen der Eltern scheint in der Gruppe der vermeidend gebundenen pflegenden Kinder vergleichsweise erhöht (Markiewicz et al. 1997). Darüber hinaus scheinen erwachsene Kinder mit einem vermeidenden Bindungsstil seltener bereit zu sein, sich in der Betreuung ihrer alternden Eltern zu engagieren. Wird die elterliche Pflege dennoch übernommen, wird sie als belastender erlebt als von sicher gebundenen erwachsenen Kindern (Karantzas et al. 2010). Das Bindungsverhalten der pflegenden Kinder scheint jedoch nicht nur die Erfolgsaussichten im Umgang mit der Pflegebelastung zu beeinflussen, sondern wirkt sich auch auf die Art der geleisteten Pflege aus. So ist eine sichere Bindung der erwachsenen Kinder mit einem erhöhten $\mathrm{Ma}$ an emotionaler Unterstützung für die Eltern verbunden. Unsicher gebundene Kinder zeigen zudem eine geringere grundsätzliche Bereitschaft zur Übernahme der Pflegerolle, ängstlich gebundene vermehrt ineffektivere Pflegeleistungen (Carpenter 2001; Collins und Feeney 2000).

Ein weiterer wichtiger Faktor, der das Ausmaß des subjektiven Belastungserlebens erwachsener Kinder in der Betreuung ihrer alternden Eltern entscheidend beeinflusst, stellt der Grad der filialen Reife der betroffenen Kinder dar.

\subsubsection{Filiale Reife und filiale Angst}

Das Konstrukt der filialen Reife wurde in den 1960er Jahren von Margaret Blenkner eingeführt (Blenkner 1965). Sie kritisierte, dass bisherige Persönlichkeitstheorien die Entwicklung des Menschen mit Erreichen der Geschlechtsreife als abgeschlossen sähen, und es somit an einer Beschreibung des wichtigen Stadiums zwischen der Geschlechtsreife und dem hohen Alter fehle. In diesem Stadium würden erwachsene Kinder im Alter zwischen 40 und 50 Jahren mit der nachlassenden Leistungsfähigkeit und der zunehmenden Abhängigkeit ihrer alternden Eltern konfrontiert. Zur Beschreibung dieses Reifestadiums wählte Blenkner den Begriff „filiale Reife“ (filial maturity), der charakteristischerweise die „filiale Krise“ (filial crisis) vorausgehe. In dieser Phase kämen die erwachsenen Kinder mittleren Alters zu der Einsicht, dass ihre Eltern nicht mehr länger als „Fels in der Brandung" gesehen werden könnten, an die sich die Kinder in Zeiten emotionaler und finanzieller Schwierigkeiten wenden könnten, sondern diese nun vielmehr selber vermehrt auf Zuspruch und Unterstützung ihrer Kinder angewiesen seien (vgl. Linkersdörfer 2006). Die gelungene Bewältigung der filialen Krise führt laut Blenkner zur filialen Reife, in der sich die Kinder nach der Rebellion und 
Emanzipation der Adoleszenz und des jungen Erwachsenenalters ihren Eltern auf neue Weise - nicht mehr als Kind sondern als reifer Erwachsener - zuwendeten. Diese neue Rolle beinhalte auch eine neue Art von Liebe, die es dem filial reifen Kind ermögliche, seine Eltern nun zum ersten mal als Individuen mit eigenen Rechten, Bedürfnissen, Einschränkungen und einer eigenen Lebensgeschichte zu sehen, die die Eltern zu den Personen gemacht haben, die sie nicht nur aktuell sondern schon lange vor der Geburt des Kindes waren (vgl. Linkersdörfer 2006 und Stiens 2000). Filiale Reife führe zum Annehmen und erfolgreichen Erfüllen der „filialen Aufgabe“ (filial task), der gesellschaftlich geforderten Unterstützung der alternden Eltern, und sei gleichzeitig für die erwachsenen Kinder durch die Identifikation mit den Eltern eine Vorbereitung auf das eigenen Altern. Blenkner hebt in ihren Ausführungen hervor, dass hierbei kein Rollentausch zwischen alternden Eltern und filial reifen Kindern stattfindet. Die filiale Krise markiere zwar das Ende der Kindheit, die erwachsenen Kinder könnten ihren Eltern gegenüber jedoch immer nur eine filiale Rolle einnehmen, die durch das sich Verlassen auf die Kinder und die Verlässlichkeit der erwachsenen Kinder charakterisiert werde („,being depended on and therefore being dependable“, Blenkner 1965, S. 57; vgl. auch Linkersdörfer 2006).

In Anlehnung an Blenkners Konzept der filialen Reife führte Victor Cicirelli 1988 den Begriff der „filialen Angst“ (filial anxiety) ein (Cicirelli 1988). Er beschrieb filiale Angst als Stadium der Besorgnis über den zu erwartenden gesundheitlichen und geistigen Abbau der alternden Eltern und der damit verbundenen Sorge der erwachsenen Kinder, den Bedürfnissen der Eltern in Zukunft gerecht werden zu können. Das Auftreten filialer Angst sei mit der Verbundenheit des Kindes mit seinen Eltern und dem Wunsch des Kindes, den gesundheitlichen Zustand der Eltern zu erhalten, assoziiert. Moderate Level an filialer Angst könnten hierbei zu einer stärkeren Motivation zur Übernahme der Pflegerolle führen, während sehr hohe Level eher zu einem Vermeiden betreuender Tätigkeiten führen könnten. Dabei betont Cicirelli, dass eine Person mit filialer Angst nicht notwendigerweise gleichzeitig auch filial unreif sein müsse. Er stellt das Phänomen der filialen Angst vielmehr in Zusammenhang mit Blenkners Stadium der filialen Krise und schlug daher vor, filiale Angst als indirektes Maß für filiale Reife einzusetzen.

Corinne Nydegger setzte sich 1991 kritisch mit Blenkners Konstrukt der filialen Reife auseinander. Ihrer Meinung nach sei das Erlangen der filialen Reife weder an ein spezifisches Alter noch an eine vorausgehende filiale Krise gebunden, sondern sei vielmehr ein zeitlich sehr variabler und langsamer Prozess der Entwicklung im Erwachsenenalter (Nydegger 1991), der sogar innerhalb von Familien abhängig von Geschlecht und Eltern-Kind-Bindung sehr unterschiedlich verliefe. Zudem sollten Zuneigung und Liebe nicht als Bestandteil des Modells gesehen werden, da enge emotionale Bindungen sich nicht nur positiv auf den Reifeprozess auswirkten, sondern diesen auch behindern könnten, und es darüber hinaus keinen Anhalt dafür gebe, dass filiale Reife nicht auch in Abwesenheit 
positiver Gefühle erlangt werden könne. Mit Hilfe des „Fatherhood Project“ erweiterte Nydegger Blenkners Überlegungen und beschrieb einen parallel zum filialen Reifungsprozess stattfindenden paternalen Reifungsprozess der Eltern. Diese beiden Prozesse beeinflussten sich gegenseitig zwar stark, könnten aber in unterschiedlichen Geschwindigkeiten ablaufen und das Scheitern des einen würde nicht zwangsläufig auch zum Scheitern des anderen Prozesses führen. Den Dimensionen Distanzierung (distancing) und Verständnis (comprehending) fallen dabei laut Nydegger zentrale Rollen zu (vgl. auch Linkersdörfer 2006 und Stiens 2000). Die emotionale Distanzierung bzw. Emanzipation des Kindes sei die Voraussetzung für eine objektive Betrachtung sowohl der eigenen Person als auch der Eltern. Bei erfolgreicher Distanzierung könnten die Eltern nun gelöst von der Elternrolle als eigenständige Personen wahrgenommen werden, was zum Verstehen der bzw. Verständnis für die Eltern führe. Auch der paternale Reifungsprozess beinhalte neben der Akzeptanz und der Unterstützung der filialen Lösungsbestrebungen die psychologische Distanzierung der Eltern von ihrem Kind. Indem die Eltern aktiv zuhörten und nicht versuchten, in ihrem Kind eine junge Version ihrer selbst zu erschaffen, würden die Eltern die Voraussetzung dafür schaffen, die gesellschaftliche und soziale Umwelt ihres Kindes verstehen zu lernen. Dies führe zum elterlichen Verständnis des Kindes. Bei erfolgreichem Durchlaufen des filialen und paternalen Reifungsprozesses könnten Kinder und Eltern sich nun einander neu als reife Erwachsene zuwenden und die alten Rollen hinter sich lassen.

Alfons Marcoen beschrieb filiale Reife 1993 als multidimensionales Konstrukt, in dem das filial reife Kind bereit ist, seinen Eltern freiwillig Hilfe zu leisten (filiale Hilfsbereitschaft) und ihnen motiviert durch liebevolle Gefühle (filiale Liebe) und einem Grad an Pflichtgefühl (filiale Verpflichtung) in einer auf Gegenseitigkeit beruhenden Beziehung (elterliche Besorgnis) und einem funktionierenden familiären Netzwerk (familiäre Solidarität) auch tatsächlich Hilfe leistet (filiale Hilfe), ohne allerdings die eigene Selbstständigkeit (filiale Autonomie) zu verlieren (Marcoen 1993 und 1995; vgl. Stiens 2000). Filiale Reife sei dabei keine absolute bzw. gesellschaftlich geforderte Norm bezüglich der Art und Weise sowie des Ausmaßes der geleisteten Hilfe und Unterstützung sondern ein dynamischer Zustand der erfolgreichen Adaptation an die sich stetig verändernde Situation der alternden Eltern. Als zentrale Dimensionen des Reife-Modells bezeichnete Marcoen filiale Liebe und filiales Verpflichtungsgefühl, die gleichzeitig auch die stärksten Motivatoren zur Übernahme der elterlichen Pflege seien. Ebenso sei neben diesen Faktoren eine ausgewogene Balance zwischen „togetherness“ und ,separateness“ von großer Wichtigkeit für ein gesundes Pflegeverhältnis (Marcoen 1995, S.134).

Geprägt durch seine Arbeit mit pflegenden Angehörigen von Demenzkranken stellte Jens Bruder 1988 filiale Reife im Kontext mit Pflegebeziehungen als grundsätzliche Bereitschaft des filial reifen Kindes zu einer „einseitig gewordenen Beziehung“ zu den Eltern mit „einseitiger Verantwortungsübernahme“ dar, als dessen Basis ein stabiles Bewusstsein positiver Gefühle diene (Bruder 1988, S. 101). Filial 
reifes Verhalten müsse freiwillig aus autonomer Position heraus erfolgen und als übergeordnetes Ziel die Verbesserung der Beziehung zu den Eltern und damit zugleich die Erleichterung des Zusammenlebens in einem Pflegeverhältnis haben. Hierzu gehöre neben dem Verständnis für die „,intergenerative Übertragung“ die Fähigkeit und Bereitschaft, Konflikte offen und konstruktiv auszutragen, sowie ein Interesse an der Versöhnung (Bruder 1988, S. 97). Ein weiteres Merkmal filialer Reife sei das „Einfühlungsvermögen in das Schwächerwerden der Elternperson“ und folglich die Anerkennung ihrer Defizite (Bruder 1988, S. 97), deren Leugnung durch enttäuschte - da der Situation der Eltern nicht mehr angemessene - Erwartungen eine erhöhte Pflegebelastung für die Kinder mit sich bringe. Von besonders großer Relevanz seien laut Bruder folgende drei Aspekte der filialen Reife: emotionale Autonomie, die Fähigkeit zu einem fürsorglich-autoritären Führungsstil und die Kontrollfähigkeit unangemessener Schuldgefühle (vgl. auch Linkersdörfer 2006 und Stiens 2000). Der Grad an emotionaler Autonomie eines pflegenden Kindes habe eine zentrale Bedeutung in der Pflegesituation, da „nur eine emotional selbständige Person [...] den schrittweisen Verlust ihrer Mutter oder ihres Vaters pflegend erleben und darüber trauern“ könne, ohne dabei „handlungsunfähig“ zu werden (Bruder 1988, S. 97). Bei unzureichend emotional autonomen Kindern bestehe die Gefahr des Wiederauflebens alter Konflikte verbunden mit dem Entstehen von Spannungen durch die „Umkehrung des ursprünglichen Macht- und Autoritätsgefälles“, was bei noch kindlich an die Eltern gebundenen erwachsenen Kindern häufig „eine Mischung aus Aggressionen und Schuldgefühlen“ hervorrufe (Bruder 1988, S. 98). Emotionale Unabhängigkeit des pflegenden Kindes stelle zudem eine Voraussetzung für einen fürsorglich-autoritären Umgang mit dem auf die Pflege angewiesenen Elternteil dar. Filiale Reife beinhalte laut Bruder auch die Fähigkeit, dem dementiell veränderten Verhalten des Elternteils beizeiten durch autoritär anmutende Maßnahmen Grenzen zu setzen. Die andernfalls aus diesem Störverhalten resultierenden Kränkungen und Konflikte könnten sonst die positiven Verbundenheitsgefühle zu den Eltern überschatten und auf Seiten des Kindes zu aggressiven Wünschen und Schuldgefühlen führen. Die Kontrollfähigkeit unangemessener Schuldgefühle stellt nun nach Bruders Verständnis die dritte wichtige Dimension der filialen Reife dar. Übersteigerte Schuldgefühle könnten bei dem betroffenen Kind zu einer permanenten Selbstabwertung mit einem konsekutiven Verlust des Selbstvertrauens und der Kraft für die alltäglichen Aufgaben führen. Dies ziehe den Verlust der Entscheidungsfreiheit und des sicheren Gefühls für den angemessenen Einsatz im elterlichen Pflegeverhältnis nach sich (Bruder 1988).

Filiale Reife kann zusammenfassend als Stadium in der Entwicklung erwachsener Kinder verstanden werden, in dem die Kinder ein neues Gleichgewicht in der Beziehung zu ihren alternden Eltern finden müssen. Filial reif zu sein bedeutet demnach, die Fähigkeit zu besitzen, ein ausgewogenes Verhältnis zwischen der Übernahme von Verantwortung für die Eltern und der Berücksichtigung eigener Interessen und Ziele schaffen zu können, ohne Schuldgefühle zu entwickeln. Voraussetzungen hierfür sind neben der Wahrnehmung der Eltern als eigenständige Personen mit zunehmenden 
Einschränkungen die emotionale Autonomie der erwachsenen Kinder und ihre damit verbundene Handlungsfähigkeit in der Betreuung der alternden Eltern.

\subsection{Zielsetzung und Arbeitshypothesen}

Eine chronische Erkrankung der Eltern geht mit dem erhöhten Risiko einer zukünftigen Pflegebedürftigkeit einher und kann so für die erwachsenen Kinder die Zuspitzung der filialen Krise bedeuten. In der Eltern-Kind-Beziehung entsteht nun eine zunehmende Einseitigkeit, die die Kinder zwingt, vermehrt Verantwortung für ihre alternden Eltern zu übernehmen. Wie bereits beschrieben führt dies zumeist zu einer erhöhten Belastung der pflegenden Kinder. Um die Situation dieser Angehörigen in der häuslichen Pflege zu verbessern, wird vermehrt nach protektiven Faktoren gesucht, die es ermöglichen, in der Angehörigenbetreuung Risikopersonen besser beraten und Copingstrategien gezielter fördern zu können. Grundlage vieler Beratungen ist bis heute das in den 60er Jahren von Margaret Blenkner entwickelte Konstrukt der filialen Reife (Blenkner 1965). Zur Erfassung der filialen Reife sind bislang zwei Verfahren bekannt: zum einen die von Marcoen entwickelte Filiale-Reife-Skala A (Marcoen 1993), die schon in zwei Voruntersuchungen eingesetzt wurde, und zum anderen die Filial Anxiety Scale von Cicirelli, die filiale Angst als indirektes Maß für filiale Reife misst (Cicirelli 1988).

Ziel der vorliegenden Studie ist es, eine aussagekräftige und effektive Skala zur Messung der Filialen Reife zu finden. Zudem soll der Zusammenhang zwischen der filialen Reife und dem Belastungserleben der betreuenden Kinder von Parkinsonpatienten untersucht werden. Auch wird der Frage nachgegangen, inwieweit sich Belastungs- und Beziehungserleben auf die subjektive Schlafqualität der Studienteilnehmer auswirkt.

Hierzu sind fünf Arbeitshypothesen formuliert worden:

$\mathrm{Da}$ es in der Literatur eindeutige Hinweise darauf gibt, dass die filiale Reife erwachsener Kinder Auswirkungen auf den gelungenen Umgang mit der Pflegesituation der alternden Eltern hat, besagt die erste Hypothese, dass eine hohe filiale Reife zu einem verminderten Belastungserleben des betreuenden Kindes führt. Es soll geprüft werden, ob hohe Werte für filiale Reife einen mindernden Effekt auf das Ausmaß psychischer und physischer Belastungssymptome der Pflegenden haben.

Zudem werden die beiden zur Verfügung stehenden Messinstrumente für filiale Reife hinsichtlich ihrer Aussagekraft zum Belastungserleben verglichen. Es wird davon ausgegangen, dass die Filial Anxiety Scale besser mit dem Belastungserleben des betreuenden Kindes korreliert als die FilialeReife-Skala A (

In diesem Zusammenhang soll auch der Einfluss der subjektiven Belastung auf die Schlafqualität der Betroffenen untersucht werden. Die dritte Hypothese besagt daher, dass Schlafstörungen sowohl bei 
den betreuenden Kindern als auch bei den Patienten zu einer Verstärkung des Belastungserlebens und einer Einschränkung der gesundheitsbezogenen Lebensqualität führen.

Nach der vierten Hypothese, korreliert die Pflegebedürftigkeit des Parkinsonpatienten positiv mit dem Belastungserleben des betreuenden Angehörigen. Hierzu werden die funktionellen und seelischen Beeinträchtigungen der Patienten in den Zusammenhang mit den subjektiven Werten für das Belastungserleben und dem allgemeinen Gesundheitszustand der betreuenden Kinder gestellt und auf signifikante Korrelationen hin überprüft.

In der Annahme, dass eine hohe filiale Reife sich auch über das Ausmaß der subjektiven Belastung durch die Pflege in der Schlafqualität der betreuenden Angehörigen widerspiegelt, lautet die fünfte Hypothese: eine hohe filiale Reife der pflegenden Kinder und eine gute Schlafqualität korrelieren positiv miteinander. 


\section{Material und Methoden}

\subsection{Zielgruppe}

An der vorliegenden Untersuchung nahmen Parkinson-Patienten und deren erwachsene Töchter und Söhne teil, die sich in der Betreuung ihres erkrankten Elternteils engagierten. Sowohl die in die Studie aufgenommenen betreuenden Kinder als auch die Patienten wurden mit Hilfe verschiedener Fragebögen interviewt.

Voraussetzung für den Einschluss des Patienten in diese Untersuchung war die fachärztlich gesicherte Diagnose eines idiopathischen Parkinsonsyndroms (iPS). Zum Ausschluss einer im Krankheitsverlauf bereits eingetretenen dementiellen Entwicklung wurde bei jedem Patienten ein Mini-Mental StatusTest durchgeführt, bei dem mindestens 25 von 30 möglichen Punkten erreicht werden mussten (s.u.).

Bei den erwachsenen Kindern musste als Voraussetzung für einen erfolgreichen Studieneinschluss neben einem Mindestalter von 18 Jahren ein regelmäßiger persönlicher oder telefonischer Kontakt zu ihrem an dem idiopathischen Parkinsonsyndrom erkrankten Elternteil bestehen. Dieser Kontakt musste kein direktes körperliches Pflegeverhältnis sein; Voraussetzung war jedoch, dass sich das Kind in persönlichen sowie gesundheitlichen Belangen des erkrankten Elternteils angesprochen fühlte.

Eingeschlossen wurden sowohl zum Zeitpunkt der Befragung stationäre als auch ambulante Patienten und ihre Angehörigen. Pro Patient wurde ein betreuendes Kind in die Untersuchung aufgenommen (= Betreuer).

Betreuende Schwiegerkinder konnten für diese Untersuchung nicht berücksichtigt werden, genauso wenig wie kinderlose Parkinsonpatienten.

Darüber hinaus wurde für die Studie eine Kontrollgruppe mit erwachsenen Kindern von gesunden alten Menschen interviewt, die entsprechend Alter, Geschlecht und Bildungs- bzw. Berufsstand auf die Betreuergruppe abgestimmt wurde. Diese Kontrollpersonen mussten noch mindestens ein lebendes Elternteil haben, das nicht schwer chronisch krank oder anderweitig pflegebedürftig war. Elterliche Erkrankungen, die zum Ausschluss einer Kontrollperson führten, waren beispielsweise eine Demenz, neurodegenerative Erkrankungen jeglicher Art oder schwere akute Krebserkrankungen. Kontrollpersonen mit einem sich bei guter Gesundheit befindlichen Elternteil, bei denen das andere Elternteil zuvor aber pflegebedürftig und bereits verstorben war, konnten ebenfalls nicht für diese Untersuchung berücksichtigt werden.

\subsection{Rekrutierung der Probanden}

Die Rekrutierung der Studienteilnehmer erfolgte zum einen über die Ärzte der Parkinsonambulanz der Abteilung Klinische Neurophysiologie des Universitätsklinikums Göttingen, die die Patienten über die Studie informierten und bei Einverständnis ihre Telefonnummern weitergaben. Zum anderen erklärte 
sich je ein niedergelassener Neurologe aus Göttingen und aus Wolfenbüttel bereit, seine Patienten von der Studie in Kenntnis zu setzen und bei Einverständnis ebenfalls die Telefonnummern zur Kontaktaufnahme weiterzuleiten. Darüber hinaus erfolgte die Rekrutierung der Probanden über die Parkinson-Fachklinik Paracelsus Elena Klinik in Kassel, in der die Patienten persönlich angesprochen und um Mitarbeit gebeten wurden.

Die Patienten wurden telefonisch über Inhalt und Umfang der Untersuchung durch die Untersucherin informiert. Erklärten sie sich mit der Teilnahme einverstanden, wurden die betreuenden Kinder der Patienten ebenfalls telefonisch über den Studieninhalt informiert und zur Mitarbeit gebeten.

Bei Einverständnis sowohl des Patienten als auch des betreuenden Kindes zur Teilnahme an der Untersuchung wurde mit ihnen ein Termin zur Durchführung des persönlichen Interviews vereinbart.

Die Gruppe der Kontrollpersonen fand sich überwiegend durch Ansprechen bekannter Personen von Seiten der Untersucherin.

Auffällig war die Tatsache, dass einige Patienten, die sich zuerst prinzipiell zur Studienteilnahme bereit erklärt hatten, dieses Einverständnis nach der Aufklärung über die ebenfalls gewünschte Mitarbeit des betreuenden Kindes mit der Begründung, ihre Kinder nicht unnötig mit ihrer Erkrankung belasten zu wollen, zurückzogen $(n=4)$.

Zudem erwähnenswert ist, dass sich die betreuenden Kinder, die ihre Eltern pflegten, im Vergleich zur Gruppe der nicht hauptsächlich betreuenden Patientenkinder in der Regel viel schneller zur Teilnahme an der Untersuchung bereit erklärten.

\subsection{Stichprobe}

Auf diese Weise konnten 50 Patienten-Betreuer-Paare für die Teilnahme an der Untersuchung gewonnen werden. Zweiunddreißig von ihnen wurden über die Parkinsonambulanz des Universitätsklinikums Göttingen rekrutiert, 15 über die Paracelsus Elena Klinik in Kassel, zwei über den niedergelassenen Neurologen in Wolfenbüttel und eines über einen niedergelassenen Neurologen in Göttingen.

Fünf weitere Patienteninterviews konnten nicht in die Untersuchung einfließen, da die betreuenden Kinder im Nachhinein keine Zeit für eine Studienteilnahme fanden.

In zwei Fällen handelte es sich bei den betreuenden Kindern nicht um leibliche Kinder des Patienten sondern um im Säuglings- bzw. Kleinkindalter angenommene Adoptivkinder. Da diese aber in beiden Fällen von den Patienten wie eigene Kinder aufgezogen worden waren, wurden sie ganz regulär mit in die Untersuchung eingeschlossen. 


\subsection{Untersuchungsmethode}

Mit Hilfe standardisierter Verfahren sollte das subjektive Belastungserleben der erwachsenen Kinder der an der Studie teilnehmenden Parkinsonpatienten erhoben werden. Hierzu wurden bei den betreuenden Kindern Faktoren der filialen Reife, die subjektive Belastung durch die Betreuung und die damit verbundene Lebensqualität ermittelt und mit der Schlafqualität sowie dem allgemeinen körperlichen und seelischen Gesundheitsbefinden der Studienteilnehmer in Verbindung gebracht. Hierbei lag besonderes Augenmerk auf der Beziehung zwischen den Faktoren der filialen Reife und dem Ausmaß der subjektiven Belastung der erwachsenen Kinder. Weitere Zielgrößen der Untersuchung waren der Gesundheitszustand und die Schlafqualität der Parkinsonpatienten, deren Auswirkung auf das Belastungserleben ihrer erwachsenen Kinder ebenfalls untersucht wurde.

Zur Ermittlung dieser Daten wurden im Patientengespräch folgende Instrumente eingesetzt:

- ein halbstandardisiertes Interview zur Anamneseerhebung

- Stadienbestimmung des idiopathischen Parkinsonsyndroms nach Hoehn \& Yahr (Hoehn und Yahr 1967)

- Unified Parkinson's Disease Rating Scale motor score = UPDRS Motorskala (Fahn et al. 1987)

- $\quad$ Mini-Mental Status-Test = MMST (Folstein et al. 1975)

- Geriatrische Depressionsskala nach Yesavage = GDS (Yesavage 1988)

- 10-Item Center for Epidemiological Studies Depression Scale = CESD-10 (Andresen et al. 1994)

- MOS Short-Form-36 Health Survey = SF-36 (Tarlov et al. 1983) [dt. Version Bullinger 1995]

- Parkinson's Disease Sleepiness Scale = PDSS (Chaudhuri et al. 2002)

- $\quad$ Epworth Sleepiness Scale = ESS (Johns 1991) [dt. Version Bloch et al. 1999]

- Pittsburgh Sleep Quality Index = PSQI (Buysse et al. 1989)

Im Gespräch mit den betreuenden Kindern und den Kontrollpersonen wurden folgende Instrumente eingesetzt:

- ein halbstandardisiertes Interview zur Anamneseerhebung

- $\quad$ Mini-Mental Status-Test = MMST (Folstein et al. 1975)

- Filial Anxiety Scale (Cicirelli et al. 1988)

- Filiale-Reife-Bogen A = LFMS-A (deutsche Übersetzung der Louvain Filial Maturity Scale A von Marcoen 1993)

- Fragebogen zur Belastung durch die Betreuung auf der Grundlage des Zarit-Burden-Interviews (Zarit et al. 1980)

- $\quad$ MOS Short-Form-36 Health Survey = SF-36 (Tarlov et al. 1983) [dt. Version Bullinger et al. 1995]

- $\quad$ 10-Item Center for Epidemiological Studies Depression Scale = CESD-10 (Andresen et al. 1994) 
- Nurses' Observation Scale for Geriatric Patients = NOSGER II (Spiegel et al. 1991)

- Epworth Sleepiness Scale = ESS (Johns 1991) [dt. Version Bloch et al. 1999]

- Pittsburgh Sleep Quality Index = PSQI (Buysse et al. 1989)

Die Patienten wurden ohne Ausnahme persönlich zum Gespräch getroffen und durch die verschiedenen Fragebögen geführt. Diese Gespräche fanden je nach Patientenwunsch im Universitätsklinikum Göttingen, in der Paracelsus Elena Klinik in Kassel oder bei den Patienten zu Hause statt.

Teilweise war ein persönliches Treffen mit den betreuenden Kindern aus zeitlichen Gründen oder aufgrund einer zu großen räumlichen Distanz nicht möglich. In diesen Fällen wurde das halbstandardisierte Anamnesegespräch/Eingangsinterview mit den Betreuern telefonisch durchgeführt, die Fragebögen wurden anschließend per Post verschickt. Den Fragebögen wurde sowohl ein Anschreiben zur nochmaligen Information als auch ein frankierter Rückumschlag beigelegt. Für eventuelle Fragen des Betreuers stand die Untersucherin telefonisch oder per Email-Kontakt zur Verfügung.

So fanden 18 Betreuergespräche persönlich statt, 32 Betreuer bekamen die Fragebögen zugeschickt und sendeten diese vollständig ausgefüllt zurück. In der Gruppe der Kontrollpersonen wurden die Gespräche in 19 Fällen persönlich geführt. Einunddreißig Kontrollpersonen bekamen die Fragebögen zugeschickt, nachdem das Anamnesegespräch zuvor telefonisch stattgefunden hatte, und sendeten diese ebenfalls vollständig ausgefüllt zurück.

\subsubsection{Anamnese}

Ziel der Anamnese war es, mit Hilfe eines halbstandardisierten Interviews das Umfeld der Betreuung sowie die familiäre Situation der Patienten und der betreuenden Kinder näher zu erfassen.

Zielgrößen im Patientengespräch waren hierbei Familienstand, Anzahl eigener Kinder, Wohnsituation, Zugehörigkeit zur Stadt- oder Landbevölkerung und Angaben zur beruflichen Situation. Darüber hinaus wurden Angaben zu einer etwaigen Einstufung in eine Pflegestufe sowie zur aktuellen Medikation, Dauer der Parkinsonerkrankung und Nebenerkrankungen erhoben.

Die Schwere der Erkrankung wurde durch die Bestimmung des Stadiums nach Hoehn \& Yahr und durch die Ermittlung der UPDRS Motorskala objektiviert (s.u.).

Vor Beginn der eigentlichen Fragebogenbefragung wurde mit jedem Patienten zum Ausschluss einer bereits im Verlauf der Parkinsonerkrankung eingetretenen dementiellen Entwicklung ein Mini-Mental Status-Test (MMST) durchgeführt (s.u.). Dieser sollte sicherstellen, dass in der vorliegenden Untersuchung tatsächlich die im Zusammenhang mit dem idiopathischen Parkinson-Syndrom entstehende Belastung und nicht die durch eine Demenz bzw. durch eine diesbezügliche CoMorbidität hervorgerufene Belastung betreuender Angehöriger gemessen wird. Darüber hinaus diente 
der Test zur Abgrenzung der an der Untersuchung teilnehmenden Patienten von einer Vergleichsstudie, die sich mit dem Belastungserleben erwachsener Kinder von Demenzerkrankten befasst. Die Bedingung zur Teilnahme an der vorliegenden Studie war ein Erreichen von mindestens 25 von 30 möglichen Punkten im MMST.

Im Anamnesegespräch mit den Betreuern waren neben den allgemeinen demographischen Angaben und eigenen Vorerkrankungen die Auskunft über eine bestehende Funktion als Hauptbetreuer und eine mögliche Unterstützung durch Dritte, sowie räumliche Nähe und Frequenz der Kontakte zum erkrankten Elternteil von Bedeutung. In den persönlich geführten Interviews wurde auch hier mit jedem Teilnehmer ein MMST durchgeführt. In den Fällen, in denen das Eingangsgespräch telefonisch ablief und die Fragebögen anschließend verschickt wurden, entfiel der MMST (n=32).

Das mit den Kontrollpersonen geführte Interview entsprach mit wenigen Ausnahmen dem der betreuenden Kinder der Parkinsonpatienten. Die Fragen nach einer Funktion als Hauptbetreuer und einer Unterstützung durch Dritte entfielen. Dafür wurde hier zusätzlich das Alter der Eltern der Kontrollpersonen erfasst. Die gesundheitliche Situation der Eltern im Hinblick auf eine mögliche schwere chronische Erkrankung bzw. eine anderweitige Pflegebedürftigkeit, die zum Ausschluss der Kontrollperson an dieser Untersuchung geführt hätte, war jeweils schon vor Studieneinschluss der entsprechenden Kontrollperson erfasst worden (s.o.).

\subsubsection{Stadienbestimmung des Parkinson-Syndroms nach Hoehn \& Yahr}

Margaret Hoehn und Melvin Yahr formulierten 1967 eine Einteilung der Stadien des idiopathischen Parkinson-Syndroms, die die Erkrankung in fünf Abschnitte gliedert (Hoehn und Yahr 1967). Während sich die Symptome im Stadium I ohne oder allenfalls mit minimaler funktionaler Beeinträchtigung nur unilateral zeigen, ist das Stadium II durch eine bilaterale Ausprägung der Symptome charakterisiert. Das dritte Stadium beschreibt eine mäßig schwere Beeinträchtigung des Patienten durch die Erkrankung mit Gleichgewichtstörungen beim Gehen und Stehen. Im Stadium IV ist der Patient aufgrund einer starken Symptomatologie mit Rigidität, Bradykinese und stark beeinträchtigter Gehfähigkeit auf fremde Hilfe angewiesen. Stadium V ist schließlich durch eine völlige Behinderung des Patienten charakterisiert, der an Rollstuhl oder Bett gefesselt ist und konstante Betreuung benötigt. 


\subsubsection{Unified Parkinson's Disease Rating Scale motor score (UPDRS Motorskala)}

Fahn et al. entwickelten 1987 die Unified Parkinson's Disease Rating Scale und etablierten damit eine einheitliche Bewertungsskala für die Symptomschwere des Parkinson-Syndroms (Fahn et al. 1987). Die Skala ist in vier verschiedene Bereiche gegliedert, die sich mit den am häufigsten mit dieser Erkrankung assoziierten Funktionseinschränkungen befassen: Teil I - Verhalten (Kognition, Stimmung, Psyche, Motivation); Teil II - Aktivitäten des täglichen Lebens; Teil III - motorische Untersuchung; Teil IV - Komplikationen der Behandlung. Zusätzlich werden im Teil V das Stadium nach Hoehn \& Yahr (Hoehn und Yahr 1967) und im Teil VI die Einteilung nach Schwab und England (Schwab und England 1969) erfasst.

Die UPDRS ist gut validiert und ihr Einsatz in Klinik und Forschung weit verbreitet (Martínez-Martín et al. 1994; Richards et al. 1994; Stebbins et al. 1998).

Für diese Studie wurde lediglich die motorische Untersuchung der UPDRS genutzt (Teil III), die dem Untersucher eine einfache Quantifizierung von Art, Anzahl und Schwere extrapyramidaler Symptome ermöglicht. In achtzehn Bereichen befasst sie sich mit dem Sprechen, dem Gesichtsausdruck, Ruhetremor (Gesicht und Extremitäten), Aktions- oder Haltetremor der Hände, Rigidität (Nacken und Extremitäten), schnellen Finger- und Handbewegungen, der Agilität der Beine, dem unassistierten Aufstehen vom Stuhl, der Körperhaltung, dem Gang, der posterioren Stabilität und der allgemeinen Bradykinese des Patienten. Für jeden Bereich wird vom Untersucher anhand einer 5-Stufen-Skala die Symptomschwere eingeschätzt, wobei 0 die Abwesenheit und 4 die maximale Ausprägung des entsprechenden Symptoms charakterisiert. Der maximal erreichbare Punktwert beträgt 108 Punkte.

\subsubsection{Mini-Mental Status-Test (MMST)}

Der 1975 von Folstein und Mitarbeitern entwickelte Mini-Mental Status-Test dient der schnellen und gezielten Untersuchung der kognitiven Funktionen (Folstein et al. 1975). Er beinhaltet 30 Fragen zu Orientierung, Merkfähigkeit, Aufmerksamkeit, Erinnerungsfähigkeit, Sprache und praktischen Funktionen des Befragten. Da für jede richtige Antwort bzw. korrekte Ausführung der praktischen Anforderungen jeweils ein Punkt vergeben wird, kann ein möglicher Gesamtwert von 30 Punkten erreicht werden. Ein relativ hoher Wert spricht somit für keine bzw. eine nur milde, ein niedriger Wert für eine höhergradige Gedächtnisstörung.

Der MMST ist in Klinik und Forschung weit verbreitet und stellt ein gutes Screening-Instrument zur Erkennung von geistigen Leistungseinschränkungen dar. Zudem wird er als Verlaufsparameter bei dementiellen Erkrankungen eingesetzt. 


\subsubsection{Geriatrische Depressionsskala nach Yesavage (GDS)}

Yesavage et al. entwickelten 1983 die Geriatrische Depressionsskala, die der Erfassung depressiver Symptome von alten, kognitiv gesunden Menschen dient (Yesavage et al. 1983). Die 30 Fragen der Skala zielen dabei eher auf die gehäuft im Alter im Vordergrund stehenden psychologischen Aspekte der Depression ab und befassen sich nicht so sehr mit somatischen Symptomen, die vergleichsweise häufiger bei Depressionen im jungen und mittleren Lebensalter auftreten.

Für die vorliegende Untersuchung wurde eine kürzere Form der GDS genutzt, die 1986 von Sheik und Yesavage beschrieben wurde (Sheik und Yesavage 1986) und die bei vergleichbarer Sensitivität und Spezifität lediglich 15 der ursprünglich 30 Fragen der Skala beinhaltet (Burke et al. 1991; Lyness et al. 1997). Der Fragebogen ist im ja/nein-Format aufgebaut und der Patient wird gebeten, die Fragen hinsichtlich ihres Zutreffens innerhalb der letzten Woche zu beantworten. Zehn dieser Fragen weisen bei positiver, fünf bei negativer Beantwortung auf das Vorhandensein depressiver Symptome hin. Jeweils ein Punkt wird für eine ein depressives Symptom bestätigende Antwort gegeben, wobei der Grenzwert der 15-Fragen-Version bei 5 Punkten liegt.

\subsubsection{0-Item Center for Epidemiological Studies Depression Scale (CESD-10)}

Die von Radloff 1977 entwickelte Center for Epidemiological Studies Depression Scale stellt ein einfaches und leicht zu handhabendes Messinstrument zur Erfassung depressiver Symptome des älteren Menschen dar (Radloff 1977). Verschiedene Kurzformen der CESD sind seitdem beschrieben (Kohout et al. 1993), die hinsichtlich ihrer Reliabilität und Validität mit der Originalversion vergleichbar sind (Irwin et al. 1999). In der vorliegenden Untersuchung kam anstelle der ursprünglichen Skala mit 20 Fragen eine 10-Fragen-Version (CESD-10) zum Einsatz. Der Befragte wird gebeten, die Aussagen hinsichtlich des Auftretens der beschriebenen depressiven Symptome innerhalb der letzten Woche auf einer Skala von 0 (,kaum oder überhaupt nicht“) bis 3 („,meistens oder die ganze Zeit“) zu beurteilen. Die Auswertung des Bogens erfolgt durch die Summation der jeweiligen Werte der Einzelantworten. Der Bereich möglicher zu erreichender Gesamtwerte liegt zwischen 0 und 30. Hohe Werte sprechen für eine stärkere Depressivität des Befragten (Andresen et al. 1994).

Obwohl die CESD ursprünglich zur Erfassung depressiver Symptome alter Menschen entwickelt wurde, haben verschiedene Untersuchungen vergleichbare Ergebnisse bei der Anwendung des Fragebogens bei Erwachsenen im jungen und mittleren Alter ergeben (Irwin et al. 1999; Radloff 1977; Radloff und Teri 1986). Aus diesem Grund kam die CESD-10 in der vorliegenden Studie nicht nur bei den Parkinsonpatienten, sondern auch in der Betreuer- sowie der Kontrollgruppe als ScreeningInstrument für depressive Symptome zum Einsatz. 


\subsubsection{Short-Form-36 Health Survey (SF-36)}

Der Short-Form-36 Health Survey wurde aus Daten der Medical Outcome Study (MOS) entwickelt und dient als krankheitsübergreifendes Messinstrument zur Erfassung der gesundheitsbezogenen Lebensqualität der Befragten (Tarlov et al. 1989). Im Rahmen des International Quality of Life Assessment Project (IQOLA) wurde der Fragebogen 1995 von Bullinger und Mitarbeitern in die deutsche Sprache übersetzt (Bullinger 1995; Bullinger et al. 1998). Der SF-36 umfasst 36 Fragen mit variablen Antwortmöglichkeiten, wovon sich 35 Fragen in folgende acht Subskalen einordnen lassen: körperliche Funktionsfähigkeit (KöFu), körperliche Rollenfunktion (KöRo), körperliche Schmerzen (KöSch), allgemeine Gesundheitswahrnehmung (AlGes), Vitalität (Vital), soziale Funktionsfähigkeit (SoFu), emotionale Rollenfunktion (EmRo), und psychisches Wohlbefinden (PsyWo).

Zur Auswertung des Fragebogens werden drei Fragen rekalibriert und weitere sieben umkodiert, so dass ein höherer Wert generell einem besseren Gesundheitszustand entspricht. Die Rohwerte der Subskalen werden nun durch Addition der zu den jeweiligen Rubriken gehörenden Fragen errechnet. Mit Hilfe eines vorgegebenen Algorithmus werden als letzter Schritt die unterschiedlichen Reichweiten der Subskalen einander angeglichen, indem die Skalenrohwerte zu einer linearen 0-100Skala transformiert werden. Der Wert 100 steht hierbei für den bestmöglichsten Gesundheitszustand, ein Wert von 0 für den schlechtesten. Darüber hinaus beinhaltet der SF-36 eine Frage zur Gesundheitsveränderung, die nicht mit in eine der oben genannten Rubriken einfließt. Genauso wenig werden hier Rohwerte oder transformierte Skalenwerte errechnet sondern lediglich die prozentuale Häufigkeit jeder Antwortmöglichkeit analysiert.

Der SF-36 ist in zahlreichen Studien auf seine Reliabilität und Validität geprüft worden und ist für den Einsatz bei Erwachsenen und Jugendlichen über 14 Jahren geeignet (Hagell et al. 2008; McHorney et al. 1993 und 1994; Ware und Sherbourne 1992).

\subsubsection{Parkinson's Disease Sleepiness Scale (PDSS)}

Chaudhuri et al. entwickelten 2002 die Parkinson Disease Sleepiness Scale, die ein einfaches Instrument zur Erfassung von Schlafstörungen bei Parkinsonpatienten darstellt (Chaudhuri et al. 2002). Mit Hilfe von visuellen Analogskalen werden 15 Fragen zu häufig beklagten schlafassoziierten Symptomen beim M. Parkinson abgefragt. Der Patient wird aufgefordert, den Schweregrad der nächtlichen Beschwerden hinsichtlich seiner innerhalb der letzten Woche gemachten Erfahrungen auf einer zehn Zentimeter langen Linie zu markieren. Die Auswertung geschieht durch Distanzmessung der Antworten in 0,1cm-Abständen. Die daraus resultierenden Scores, die für jede Frage zwischen 0 (schwere Symptomatik) und 10 (Symptomfreiheit) liegen können, werden dann zu einem Gesamtwert summiert. Dieser Gesamtscore der PDSS kann Werte im Bereich von 0 bis 150 annehmen, wobei geringere PDSS-Werte generell für einen schlechteren Schlaf des Patienten sprechen. 
Die PDSS ist gut validiert (Chaudhuri et al. 2002; Happe et al. 2004) und ihr Nutzen vor dem Hintergrund ihrer leichten Handhabung und guten Verständlichkeit für Klinik und Forschung gut belegt.

\subsubsection{Epworth Sleepiness Scale (ESS)}

Um eine Methode zur Messung der allgemeinen Tagesschläfrigkeit von Patienten zu erhalten, entwickelte Murray W. Johns 1991 in einer Studie mit 150 Patienten mit Schlafproblemen und 30 gesunden Kontrollpersonen die Epworth Sleepiness Scale (Johns 1991). In dem Fragebogen sind acht verschiedene Situationen des täglichen Lebens wie beispielsweise Lesen, Fernsehen und Autofahren dargestellt. Der Patient wird gebeten, diese Situationen hinsichtlich seines eigenen Risikos, in eben diesen Situationen einzunicken, zu bewerten. Die Betonung liegt hierbei eindeutig auf dem „einnicken“ bzw. „einschlafen“, nicht auf lediglich „müde sein“. Die Bewertung der Situationen erfolgt anhand einer Skala von 0 „würde niemals einnicken“ bis 3 ,hohe Wahrscheinlichkeit einzunicken“, basierend auf dem normalen Alltagsleben des Patienten in der letzten Zeit. Die Auswertung des ESS erfolgt durch die Bildung eines Summenscores aus den Werten der zu beurteilenden Situationen. Dieser Summenscore kann Werte zwischen 0 und 24 betragen, wobei ein höherer Wert für eine höhere subjektive Tagesmüdigkeit spricht.

Der ESS wurde 1998 von Bloch et al. für den deutschen Sprachraum übersetzt und ist ebenso wie das englische Original gut validiert (Bloch et al. 1998). Die einfache Struktur und Anwendung des Fragebogens haben die Epworth Sleepiness Scale seitdem zu einem guten Messinstrument der subjektiven Tagesschläfrigkeit gemacht und bedingen ihren weit verbreiteten Einsatz in Klinik und Forschung.

\subsubsection{Pittsburgh Sleep Quality Index (PSQI)}

Der Pittsburgh Sleep Quality Index wurde 1989 von Buysse et al. zur Erfassung der Schlafqualität in den letzten vier Wochen entwickelt und umfasst neben 19 Fragen zur Selbsteinschätzung fünf Fragen zur Fremdbeurteilung durch den Partner oder Mitbewohner (Buysse et al. 1989). Letztere fünf Fragen sind ausschließlich für den klinischen Gebrauch bestimmt und werden in der standardisierten Auswertung des Fragebogens nicht berücksichtigt, genauso wenig die 19. Frage nach einem Partner bzw. Mitbewohner. Die verbleibenden 18 Fragen werden für die quantitative Auswertung den folgenden sieben Komponenten mit einem jeweiligen Wertebereich von 0 bis 3 zugeordnet: subjektive Schlafqualität, Schlaflatenz, Schlafdauer, Schlafeffizienz, Schlafstörungen, Schlafmittelkonsum und Tagesmüdigkeit. Der Gesamtwert der PSQI ergibt sich aus der Summe der Komponentenscores und kann so Werte zwischen 0 und 21 annehmen. Eine Normierung im eigentlichen Sinne ist für den PSQI nicht beschrieben. Generell entsprechen aber höhere Werte einer schlechteren Schlafqualität. Ein von Buysse et al. empirisch ermittelter Cut-off-Wert von 5 trennt Schlafgesunde von Schlafgestörten. 
Der PSQI ist in verschiedenen Studien auf seine Reliabilität und Validität geprüft worden und stellt eine einfache sowie effektive Methode zur Erfassung der subjektiven Schlafqualität außerhalb des Schlaflabors dar (Backhaus et al. 2002; Buysse et al. 1989; Carpenter und Andrykowski 1998; Gentili et al. 1995).

\subsubsection{Filial Anxiety Scale (FAS)}

Unter der Annahme, filiale Angst als indirektes Maß für filiale Reife nutzen zu können, entwickelte Cicirelli 1988 die Filial Anxiety Scale (Cicirelli 1988). Hierzu legte er einer Gruppe von 71 durchschnittlich 46-jährigen Kindern gesunder alter Eltern einen dreizehn Aussagen umfassenden Fragebogen zur elterlichen Beziehung vor. Zur Bewertung dieser Aussagen diente eine 5-stufige Antwortskala von „überhaupt nicht zutreffend“ (1) bis „sehr zutreffend“ (5). Diese Ergebnisse wurden zu zwei Subscalen (FAA und FAB) mit sieben bzw. sechs zugehörigen Aussagen zusammengefasst. Die Filial Anxiety Scale A umfasst die Aussagen 1 bis 7, die die Sorge der Kinder über die eigene Fähigkeit, der erwarteten Hilfsbedürftigkeit der alternden Eltern entsprechen zu können, beschreibt. Die FAB wird aus den Aussagen 8 bis 13 ermittelt und befasst sich inhaltlich mit der Sorge des Kindes um den abnehmenden gesundheitlichen Zustand der Eltern und der damit verbundenen zu erwartenden Hilfsbedürftigkeit. Die Summe der Einzelwerte der zu jeder Subskala gehörenden Aussagen bildet den jeweiligen Gesamtwert der Unterskalen. Für die FAA können dementsprechend Werte zwischen 7 und 35 erreicht werden, für die FAB Werte zwischen 6 und 30. Hohe Skalenwerte repräsentieren ein hohes $\mathrm{Maß}$ an filialer Angst.

Reliabilität und Validität der Filial Anxiety Scale sind in weiteren Untersuchungen bestätigt worden (Cicirelli 1988; Murray et al. 1995 und 1996). Bislang wurde die Skala allerdings nur vereinzelt im Kontext von Pflegebeziehungen eingesetzt (vgl. Laditka und Pappas-Rogich 2001, Schmidt 2009).

\subsubsection{Louvain Filial Maturity Scale A (LFMS-A)}

Die Louvain Filial Maturity Scale wurde 1993 von Marcoen entwickelt, um das von Blenkner entwickelte Konstrukt der Filialen Reife für die Forschung greifbarer zu machen. Als psychometrisches Instrument dient die Skala der Beschreibung der Art der Beziehung erwachsener Kinder zu ihren alternden Eltern (Marcoen 1993 und 1995).

Marcoen legte hierzu 298 Probanden einen Fragebogen mit insgesamt 100 Aussagen vor, die von den Probanden auf einer Likert-Skala von 1 (überhaupt nicht passend) bis 7 (sehr gut passend) in Bezug auf die eigene Beziehung zu ihren Eltern bewertet werden sollten. Diese Ergebnisse wurden zu zwei verschiedenen Skalen (LFMS-A und LFMS-B) mit sieben bzw. vier Unterskalen zusammengefasst. Skala A umfasst hierbei die Faktoren filiale Liebe (FL), filiales Verpflichtungsgefühl (FO), filiale Hilfsbereitschaft (FHN), filiale Hilfe (FH), filiale Autonomie (FO), elterliche Besorgnis (PC) und familiäre Solidarität (FS) und die Skala B die Faktoren filiale Hilfe, filiale Liebe, elterliches 
Unverständnis und filiale Autonomie. Der für jede Subskala aus der Summe der zugehörigen Einzelwerte ermittelte Wert wird durch die Anzahl der in die Subskala einfließenden Aussagen geteilt. So erhält man für jede Subskala einen möglichen Gesamtwert zwischen 1 und 7.

Da sich der Grad der filialen Reife aufgrund der Komplexität und des multidimensionalen Charakters des Konstrukts (Marcoen 1995) jedoch nicht durch einen Gesamtwert ausdrücken lässt, muss bewusst sein, dass die verschiedenen und sich gegenseitig beeinflussenden Faktoren und deren Ausprägung Spielraum bezüglich der Gewichtung der einzelnen Faktoren in der Auswertung zulassen.

Im Rahmen zweier Voruntersuchungen (Linkersdörfer 2006; Stiens 2000) wurde die deutsche Übersetzung der Louvain Filial Maturity Scale pflegenden Kindern von Demenzkranken vorgelegt und so erstmals ihre praktische Anwendbarkeit im Kontext von Pflegebeziehungen getestet. Da sich hierbei Hinweise auf eine größere Aussagekraft der 81 Aussagen beinhaltenden LFMS-A bezüglich der filialen Reife der betreuenden Angehörigen ergaben, wurde für die vorliegende Studie ausschließlich diese Skala A verwendet.

\subsubsection{Fragebogen zur Belastung durch die Betreuung}

Der Fragebogen zur Belastung durch die Betreuung befasst sich mit Belastungsfaktoren und daraus resultierenden Symptomen in der Betreuung pflegebedürftiger alter Menschen durch ihre erwachsenen Kinder. Inhaltlich wird hierbei besonderes Augenmerk auf den Einfluss der Betreuung auf Stimmung, Gesundheit und Alltag des pflegenden erwachsenen Kindes sowie auf den emotionalen Gehalt der Pflegesituation und die Art der Beziehung zum betreuten Elternteil gelegt.

Als Vorlage für den Fragebogen diente das 1980 von Zarit et al. entwickelte Zarit Burden Interview, das ein besonders im Zusammenhang mit Belastungen in der Pflege demenzkranker alter Menschen durch ihre Angehörigen häufig verwendetes Instrument ist und sich über Jahre hinweg in Klinik und Forschung etabliert hat (Zarit et al. 1980).

Der Fragebogen zur Belastung durch die Betreuung besteht aus 29 Aussagen zur Pflegesituation, die bezüglich ihres Zutreffens mit 0 (überhaupt nicht), über 1 (ein wenig) bis hin zu 2 (ganz besonders) bewertet werden sollen. Die Auswertung erfolgt mittels Bildung des Summenwertes der Einzelantworten. Dieser kann Werte zwischen 0 und 58 annehmen und stellt somit ein quantitatives $\mathrm{Maß}$ für das durch die Betreuung empfundene Belastungserleben der betreuenden Kinder dar.

\subsubsection{Nurses' Observation Scale for Geriatric Patients (NOSGER II)}

Um ein allgemeines Messinstrument für das Maß der Einschränkung alter Menschen, die an einer Demenz, einer Depression oder einer anderen psychiatrischen Störung leiden, zu erlangen, entwickelten Spiegel und Mitarbeiter 1991 die NOSGER (Spiegel et al. 1991). Diese Skala besteht aus 30 Aussagen zu Verhaltensweisen des Betroffenen, die sich in folgende sechs Unterbereiche gliedern lassen: Gedächtnis (memory), instrumentelle Aktivitäten des täglichen Lebens (IADL), Aktivitäten des 
täglichen Lebens (selfcare), Stimmung (mood), soziales Verhalten (social behavior) und störendes Verhalten (disturbing behavior). Die bzw. der Pflegende wird gebeten, die Aussagen hinsichtlich der Häufigkeit des Auftretens dieses Verhaltens bei dem Patienten innerhalb der letzten drei Tage auf einer 5-Punkte-Skala von 1 ,immer“ bis 5 „nie“ zu beurteilen. Durch Addition der Einzelwerte der zu den jeweiligen Unterbereichen gehörenden Aussagen wird der Gesamtscore des entsprechenden Lebensbereichs ermittelt. Hierzu müssen 11 der 30 Fragen aufgrund ihrer inversen inhaltlichen Aussage genau gegensätzlich ihrer eigentlichen Skalierung gewertet werden. Da je fünf Aussagen einem Unterbereich zugeordnet sind, umfasst jede Skala einen Wertebereich von fünf bis 25 . Der Gesamtscore der NOSGER ergibt Punktwerte zwischen 30 und 150. Allgemein bezeichnet ein niedriger Bereichswert eine fehlende oder geringe Beeinträchtigung und ein hoher eine schwerwiegende Dysfunktion im diesbezüglichen Lebensbereich.

Der Fragebogen, der ursprünglich für professionelle Pflegende entwickelt wurde, ist in mehreren Studien erfolgreich auf seine Validität und Reliabilität hin geprüft worden und ist auch zur Beurteilung durch pflegende Angehörige geeignet (Spiegel et al. 1991; Tremmel und Spiegel 1993).

Die NOSGER stellt nicht nur ein fremdbeurteilendes Mittel zur Erfassung von Verhaltensauffälligkeiten alter erkrankter Menschen dar, sondern dient auch als Maß für die resultierende notwendige Betreuung und die tägliche Belastung der pflegenden Kinder. In dieser Studie wird der Fragenbogen zusätzlich auch zur Unterscheidung zwischen dem Betreuungsbedarf der Parkinsonpatienten und dem der gesunden Eltern der Kontrollpersonen und zur Abgrenzung körperlicher und kognitiver Defizite genutzt.

\subsection{Statistische Auswertung}

Die Berechnungen der Ergebnisse wurden in Zusammenarbeit mit der Abteilung Medizinische Statistik der Georg-August Universität Göttingen mit Hilfe des Computerprogramms „Statistica“ durchgeführt. Da die Daten in Form von Scores erhoben wurden und somit keine Normalverteilung vorlag, konnten zur Berechnung nur nonparametrische Analysen angewandt werden. Die Korrelationen zwischen den Werten der verschiedenen Fragebögen wurde mittels Spearman's R berechnet und in Form von Scatterplots graphisch dargestellt und gesichert. Der Wilcoxon-MannWhitney-U-Test kam zur Identifizierung von Unterschieden zwischen zwei Gruppen innerhalb eines Fragebogens zum Einsatz. Die graphische Darstellung dieser Ergebnisse erfolgte mittels Boxplots, Säulendiagrammen und Pie-Charts.

Da sich bei den Berechnungen mitunter deutliche Unterschiede zwischen männlichen und weiblichen Probanden ergaben, wurden alle Ergebnisse geschlechtsspezifisch getrennt berechnet und dargestellt. Zusätzlich fand in einigen Fällen eine getrennte Darstellung der Ergebnisse von hauptsächlich betreuenden und nicht-hauptsächlich betreuenden erwachsenen Kindern statt. 
Für die gesamten statistischen Berechnungen wurde das Signifikanzniveau von $\mathrm{p}<0,05$ angestrebt. Da jedoch die Größen einzelner Untergruppen in der vorliegenden Arbeit teilweise recht klein waren, wurden im Ergebnisteil unter Angabe der entsprechenden Rangkorrelationen und p-Werte vereinzelt auch nicht-signifikante Unterschiede dargestellt. 


\section{Ergebnisse}

Die Stichprobe für diese Untersuchung setzt sich aus 50 Patienten-Betreuer-Paaren und 50 Kontrollpersonen-Paaren zusammen.

32 Patienten konnten über die Parkinsonambulanz des Universitätsklinikums Göttingen, 15 über die Paracelsus-Elena-Klinik in Kassel, zwei über den niedergelassenen Neurologen in Wolfenbüttel und ein Patient über den niedergelassenen Neurologen in Göttingen rekrutiert werden.

In zwei Fällen handelt es sich bei den betreuenden Kindern nicht um leibliche Kinder der Patientinnen sondern um im Säuglings- bzw. Kleinkindalter angenommene Adoptivkinder. Da diese aber in beiden Fällen von den Patientinnen als eigene Kinder aufgezogen wurden, wurden sie in die Untersuchung mit eingeschlossen. Fünf weitere Patienteninterviews konnten nicht in die Untersuchung einfließen, da die betreuenden Kinder im Nachhinein keine Zeit für eine Studienteilnahme fanden.

In die Gruppe der Kontrollpersonen wurden Söhne und Töchter gesunder älterer Menschen aufgenommen, die in den Charakteristika Geschlecht, Alter (+/- 2 Jahre) und Bildungs- bzw. Berufsstand mit der Betreuergruppe gematcht wurden. Die unterschiedlichen Berufstände wurden hierzu folgendermaßen zusammengefasst: Arbeiter, einfache Angestellte und Beamte im einfachen und mittleren Dienst zu einer Gruppe, sowie leitende Angestellte, Beamte im gehobenen und höheren Dienst und Selbständige zu einer zweiten großen Gruppe. Hausfrauen/-männer und Studierende wurden jeweils mit einer Kontrollperson aus der entsprechenden Gruppe gepaart. Frührentnerinnen der Betreuergruppe wurden entsprechend ihres höchsten Bildungsabschlusses mit Hausfrauen der Kontrollgruppe gepaart, da sie seit ihrem gesundheitsbedingten Ausscheiden aus dem Berufsleben zuhause den Aufgabenbereich einer Hausfrau übernommen haben.

Diese Vorgehensweise sollte dazu dienen, dass sich Betreuer und Kontrollpersonen, die aus dem privaten Umfeld der Untersucherin stammen, zum Zeitpunkt der Datenerhebung in möglichst ähnlichen Lebenssituationen befanden. So konnte eine möglichst große Übereinstimmung der demographischen Faktoren zwischen der Untersuchungs- und der Kontrollgruppe gewährleistet werden.

Im Folgenden werden nun zuerst die Ergebnisse der Anamnese und Fragebögen der Parkinsonpatienten dargestellt und in einigen Fällen mit entsprechenden Werten der Referenzstudien verglichen. Anschließend findet die Darstellung der Ergebnisse der betreuenden erwachsenen Kinder statt, die mit Referenzwerten der Originalstudien bzw. Ergebnissen zweier Vorstudien von Stiens und Linkersdörfer verglichen werden. Bei diesen Vorstudien handelt es sich um wissenschaftliche Untersuchung zur filialen Reife und Pflegebelastung erwachsener Kinder von Alzheimerpatienten aus den Jahren 2000 und 2006. Am Ende des Kapitels werden zudem die Ergebnisse der Kontrollpersonen im Vergleich mit denen der Studiengruppe dargestellt. 


\subsection{Ergebnisse der Patientengruppe}

\subsubsection{Anamnese}

Die Untersuchungsstichprobe besteht aus 28 männlichen und 22 weiblichen Patienten mit idiopathischem Parkinsonsyndrom. 58\% der Patienten wurden zum Zeitpunkt der Datenerhebung ambulant, $42 \%$ stationär betreut. Die an der Untersuchung teilnehmenden Patienten sind zwischen 52 und 84 Jahren $(\mathrm{SD}=8,3)$ alt. Das mittlere Alter liegt bei 68,1 Jahren, wobei die männlichen Patienten durchschnittlich 2,5 Jahre jünger (67 Jahre) als die weiblichen Patientinnen sind (69,5 Jahre). Ein Patient ist ledig, 34 verheiratet, zwei geschieden und 13 verwitwet. 20\% der Patienten haben ein Kind, $50 \%$ zwei, 26\% drei und 4\% vier und mehr Kinder. Während 12 Patienten angeben, alleine zu leben, leben 34 mit einem Partner und 2 mit anderen Angehörigen zusammen. Eine Patientin ist in einem Altersheim untergebracht. Eine weitere Patientin lebt mit anderen Parkinsonpatienten in einer „Parkinson-WG“, in der die Mitbewohner einander entsprechend dem Grad ihrer erkrankungsbedingten Einschränkungen bei der Alltagsbewältigung helfen. 58\% der Patienten leben in der Stadt, $42 \%$ in ländlichen Regionen.

Zu 90\% sind die Patienten zum Zeitpunkt der Studienteilnahme berentet bzw. pensioniert. Drei Patienten sind nach wie vor berufstätig, zwei davon als einfache Angestellte, einer als Selbständiger. Zwei Patientinnen sind Hausfrauen.

Die Erstdiagnose des idiopathischen Parkinsonsyndroms wurden bei den teilnehmenden Patienten zwischen 1980 und 2007 (Median 1997) in einem mittleren Alter bei Diagnosestellung von 59,6 Jahren (Min 38 Jahre, Max 84 Jahre, SD=10,2) gestellt. Die Dauer der Parkinsonerkrankung liegt durchschnittlich bei 8,5 Jahren. Die eingangs erhobenen Werte der UPDRS-Motorskala liegen zwischen 3 und 63, der Mittelwert bei 24,1, der Median bei 23 Punkten (SD=15,0). 8\% der Patienten befinden sich im H\&Y-Stadium 1, 49\% im Stadium 2, $27 \%$ im Stadium 3, 14\% im Stadium 4 und 2\% im Stadium 5 nach Hoehn und Yahr. Bezüglich der UPDRS-Werte fehlen in zwei Fällen entsprechende Angaben, bezüglich der Stadieneinteilung nach Hoehn und Yahr in einem Fall. Während 35 Patienten in keine Pflegestufe eingestuft sind, befinden sich 11 in der Pflegestufe 1 und vier Patienten in der Pflegestufe 2.

Die Patienten nehmen zwischen 0 und sechs der verschiedenen Antiparkinson-Wirkstoffe ein (Standardabweichung 1,5). Die Tagesgesamtdosis von L-Dopa beträgt zwischen $0-800 \mathrm{mg}$. Der Mittelwert liegt bei $430 \mathrm{mg}$, der Median bei $500 \mathrm{mg}$. 33 Patienten nehmen einen Dopaminagonisten ein, sieben nehmen eine Kombination aus zwei verschiedenen Dopaminagonisten ein. Neun Patienten bekommen keinen Dopaminagonisten, in einem Fall sind die entsprechenden Angaben fehlerhaft. Die errechnete Äquivalenzdosis der eingenommenen Dopaminagonisten liegt durchschnittlich bei 3,1 (Min 0, Max 18, SD=3,6). Darüber hinaus werden drei Patienten mit Tiefenhirnstimulation behandelt. Die Anzahl der Begleiterkrankungen liegt zwischen null und elf, der Median bei vier Erkrankungen. 


\subsubsection{Mini-Mental Status-Test (MMST)}

Im Mini-Mental Status-Test werden von den Patienten Werte zwischen 25 (Mindestanforderung) und 30 Punkten (maximal zu erreichender Höchstwert) erreicht. Der Mittelwert liegt bei 28,4 Punkten, der Median bei 29 Punkten. Ein manifester dementieller Prozess im Verlauf der Parkinsonerkrankung kann demnach bei allen an der Studie teilnehmenden Patienten ausgeschlossen werden.

\subsubsection{Geriatrische Depressionsskala nach Yesavage (GDS)}

Im Folgenden werden die Ergebnisse der Geriatrischen Depressionsskala nach Yesavage dargestellt. Der Grenzwert für das Vorhandensein eines manifesten depressiven Syndroms liegt bei fünf Punkten ( $\geq 5$ ). Maximal können 15 Punkte erreicht werden.

Tabelle 2: Ergebnisse der Geriatrischen Depressionsskala nach Yesavage

\begin{tabular}{|l|l|l|r|}
\hline & $\begin{array}{l}\text { männliche } \\
\text { Patienten } \\
\mathrm{n}=28\end{array}$ & $\begin{array}{l}\text { weibliche } \\
\text { Patienten } \\
\mathrm{n}=22\end{array}$ & $\mathrm{l}$ \\
\hline GDS Yesavage & 1 & $\mathrm{n}=50$ & \\
\hline Minimum & 10 & 0 & 0 \\
\hline Maximum & 4,43 & 41 & 11 \\
\hline Mittelwert & 2,44 & 3,91 & 4,64 \\
\hline Standardabweichung & 12 & 13 & 2,86 \\
\hline Anzahl über cut-off & 42,9 & 59,1 & 25 \\
\hline Prozent über cut-off & & & 50,0 \\
\hline
\end{tabular}

Der Mittelwert der Patientinnen liegt mit 4,91 Punkten und der Median mit fünf Punkten über den entsprechenden Werten der männlichen Probanden. In Abbildung 1 sind diese Ergebnisse graphisch dargestellt. Der Wilcoxon-Mann-Whitney-U-Test ergibt für die voneinander abweichenden Werte in den geschlechtsgetrennten Untergruppen auf einem Niveau von $p<0,05$ allerdings keine statistische Signifikanz.

Insgesamt erreichen fast 60 Prozent der Patientinnen einen über dem cut-off liegenden Wert in der Geriatrischen Depressionsskala. Bei den männlichen Patienten betrifft dies nur 42,9 Prozent (Abbildung 1 und 2).

\subsubsection{0-Item Center for Epidemiological Studies Depression Scale (CESD-10)}

In Tabelle 3 werden die Ergebnisse der CESD-10 dargestellt und anhand der Vorgaben von Andresen et al. bewertet, der einen Grenzwert von $\geq 10$ Punkten ansetzt (Andresen et al. 1994). 
Tabelle 3: Ergebnisse der CESD-10

\begin{tabular}{|l|l|l|l|}
\hline & $\begin{array}{l}\text { männliche } \\
\text { Patienten } \\
\mathrm{n}=28\end{array}$ & $\begin{array}{l}\text { weibliche } \\
\text { Patienten } \\
\mathrm{n}=22\end{array}$ & $\mathrm{n}=50$ \\
\hline CESD-10 & 4 & & 2 \\
\hline Minimum & 23 & 29 & 29 \\
\hline Maximum & 9,50 & 10,73 & 10,32 \\
\hline Mittelwert & 5,12 & 6,16 & 5,55 \\
\hline Standardabweichung & 14 & 13 & 27 \\
\hline Anzahl über cut-off & 50,0 & 59,1 & 54,0 \\
\hline Prozent über cut-off & & & \\
\hline
\end{tabular}

Der Mittelwert fällt bei den männlichen Patienten niedriger aus als bei den weiblichen Studienteilnehmern. Darüber hinaus bestehen bei den Männern ein vergleichsweise deutlich geringerer Maximalwert sowie eine weniger ausgeprägte Streuung der Einzelwerte um den Median. Diese Unterschiede sind allerdings auf einem Niveau von $p<0,05$ nicht statistisch signifikant. Abbildung 3 stellt die Ergebnisse graphisch dar.

Insgesamt liegen 27 der 50 Parkinsonpatienten mit ihrem CESD-Wert über dem in der Literatur beschriebenen cut-off von $\geq 10$ Punkten. In der Gruppe der männlichen Patienten betrifft dies 50 Prozent der Probanden, in der Gruppe der weiblichen Patienten 59,1 Prozent (Abbildungen 3 und 4).

\subsubsection{Short-Form-36 Health Survey (SF-36)}

Im Folgenden werden die Ergebnisse des SF-36 dargestellt und mit den altersentsprechenden Mittelwerten des Bundes-Gesundheitssurveys von 1998 verglichen. Diese Erhebung wurde vom Robert Koch-Institut Berlin an 6964 Probanden durchgeführt und stellt eine repräsentative Normstichprobe für die Bundesrepublik Deutschland dar (Ellert und Bellach 1999). 
Tabelle 4: Mittelwerte und Standardabweichungen des SF-36 im Vergleich mit der Normstichprobe des Bundes-Gesundheitssurveys 1998 (Ellert und Bellach 1999)

\begin{tabular}{|l|r|r|r|r|r|}
\hline & $\begin{array}{l}\text { männl. } \\
\text { Patienten } \\
\mathrm{n}=28\end{array}$ & $\begin{array}{l}\text { weibl. } \\
\text { Patienten } \\
\mathrm{n}=22\end{array}$ & \multicolumn{1}{l|}{$\begin{array}{l}\text { Mlle } \\
\mathrm{n}=50\end{array}$} & $\begin{array}{l}\text { Männer } \\
\text { Normstichprobe } \\
60-69 \mathrm{~J} .\end{array}$ & $\begin{array}{l}\text { Frauen } \\
\text { Normstichprobe } \\
\text { 60-69 J. }\end{array}$ \\
\hline körperliche & 54,29 & 50,68 & 52,70 & 78,87 & 72,82 \\
Funktionsfähigkeit & $(26,86)$ & $(26,83)$ & $(26,64)$ & $(22,71)$ & $(24,93)$ \\
\hline körperliche & 25,89 & 30,68 & 28,00 & 76,52 & 73,54 \\
Rollenfunktion & $(31,54)$ & $(40,04)$ & $(35,23)$ & $(37,42)$ & $(38,28)$ \\
\hline körperliche & 56,39 & 43,55 & 50,74 & 65,00 & 60,65 \\
Schmerzen & $(26,89)$ & $(26,52)$ & $(27,23)$ & $(25,83)$ & $(26,68)$ \\
\hline allgemeine & 42,96 & 46,14 & 44,36 & 59,65 & 60,26 \\
Gesundheit & $(16,53)$ & $(18,82)$ & $(17,46)$ & $(17,06)$ & $(18,90)$ \\
\hline & 49,11 & 44,55 & 47,10 & 62,73 & 60,27 \\
Vitalität & $(17,75)$ & $(22,62)$ & $(19,95)$ & $(18,69)$ & $(17,50)$ \\
\hline Soziale & 78,13 & 69,89 & 74,50 & 87,82 & 85,37 \\
Funktionsfähigkeit & $(20,02)$ & $(25,49)$ & $(22,72)$ & $(19,31)$ & $(19,90)$ \\
\hline emotionale & 67,86 & 51,51 & 60,67 & 89,49 & 88,38 \\
Rollenfunktion & $(44,89)$ & $(44,52)$ & $(45,02)$ & $(27,78)$ & $(28,59)$ \\
\hline psychisches & 70,86 & 60,73 & 66,40 & 75,76 & 70,72 \\
Wohlbefinden & $(16,61)$ & $(24,89)$ & $(21,06)$ & $(16,22)$ & $(17,13)$ \\
\hline
\end{tabular}

Wie in Kapitel 2.4.7 beschrieben entsprechen hohe Werte der SF-36 aufgrund der Skalenkalibrierung einem guten Gesundheitszustand der Studienteilnehmer. Auch findet eine Umrechnung der Skalenrohwerte zu einer linearen 0-100-Skala statt, was den Vergleich der einzelnen Werte in Prozentpunkten ermöglicht.

Während die männlichen Patienten demnach durchschnittlich eine geringfügig schlechtere „körperliche Rollenfunktion“ und „allgemeine Gesundheit“ als die Frauen angeben, liegen die Mittelwerte der weiblichen Patienten in den Rubriken „körperliche Funktionsfähigkeit“ und „Vitalität“ geringfügig niedriger, in der „sozialen Funktionsfähigkeit“ mehr als fünf Prozentpunkte sowie in den Rubriken „emotionale Rollenfunktion“ und „psychisches Wohlbefinden“ mehr als 10 Prozentpunkte unter den entsprechenden Werten der männlichen Patienten. Zudem geben die Frauen in einem höheren Maß „körperliche Schmerzen“ an. Statistisch signifikante Unterschiede konnten jedoch auf einem Niveau von $\mathrm{p}<0,05$ nicht festgestellt werden. Eine graphische Darstellung der Ergebnisse befindet sich im Anhang (Abbildungen 5-7).

Im Vergleich mit den alters- und geschlechtsspezifischen Referenzgruppen des BundesGesundheitssurveys liegen sowohl die männlichen als auch die weiblichen Patienten in der „körperlichen Funktionsfähigkeit“ sowie der „körperlichen“ und „emotionalen Rollenfunktion“ mit mehr als 20 Punkten deutlich unter den entsprechenden Vergleichswerten. Bei den Patientinnen liegen die Mittelwerte der übrigen Rubriken allesamt mit mehr als 10 Prozentpunkten deutlich niedriger als die entsprechenden Referenzwerte. Die männlichen Patienten geben im Vergleich eine um mehr als 10 Prozentpunkte schlechtere ,allgemeine Gesundheit“ und „Vitalität“, sowie um mehr als fünf Punkte niedrigere Mittelwerte für „körperliche Schmerzen“ und „soziale Funktionsfähigkeit“ als die altersentsprechenden Probanden der Erhebung des Bundes-Gesundheitssurveys an. Aufgrund der 
Skalenkalibrierung der SF-36 zeigt dies für die Patienten ein vergleichsweise höheres Maß an körperlichen Schmerzen.

\subsubsection{Parkinson's Disease Sleep Scale (PDSS)}

Tabelle 5 zeigt die Ergebnisse des PDSS Gesamtwertes für die Gruppe der Parkinsonpatienten im Vergleich mit den Referenzwerten von Chaudhuri (Chaudhuri et al. 2002).

Tabelle 5: Ergebnisse der Parkinson's Disease Sleep Scale

\begin{tabular}{|l|r|l|r|r|r|}
\hline & \multicolumn{3}{|l|}{ Patienten } & \multicolumn{2}{l|}{ Chaudhuri } \\
\cline { 2 - 6 } & $\begin{array}{l}\text { männlich } \\
\mathrm{n}=27\end{array}$ & $\begin{array}{l}\text { weiblich } \\
\mathrm{n}=22\end{array}$ & $\begin{array}{l}\text { alle } \\
\mathrm{n}=49\end{array}$ & $\begin{array}{l}\text { Patienten } \\
\mathrm{n}=143\end{array}$ & $\begin{array}{l}\text { Kontrollen } \\
\mathrm{n}=137\end{array}$ \\
\hline PDSS gesamt & & & & & \\
\hline Minimum & 36,8 & 50,5 & 36,8 & & \\
\hline Maximum & 143,7 & 145,7 & 145,7 & & \\
\hline Mittelwerte & 100,27 & 98,80 & 99,61 & 101,1 & 120,7 \\
\hline Std.abw. & 24,77 & 24,33 & 24,33 & 21,7 & 21,0 \\
\hline
\end{tabular}

Während in der Gruppe der männlichen Patienten zwar vergleichsweise geringere Minimal- und Maximalwerte auftreten als bei den weiblichen Patienten, liegt ihr Mittelwert für die Gesamt-PDSS jedoch über dem der Patientinnen. Die Standardabweichungen sind vergleichbar. Die geschlechtsspezifischen Unterschiede in den Mittelwerten der PDSS sind auf einem Niveau von p $<0,05$ weder für den Gesamtwert noch für die im Anhang dargestellten Einzelfragen statistisch signifikant.

Im Vergleich mit der Patientengruppe von Chaudhuri et al. zeigen die Parkinsonpatienten der aktuellen Untersuchung vergleichbare Werte (Chaudhuri et al. 2002). Im Vergleich mit den Kontrollpersonen liegen die Ergebnisse sowohl der männlichen als auch der weiblichen Patienten niedriger.

\subsubsection{Epworth Sleepiness Scale (ESS)}

In Tabelle 6 werden die Ergebnisse der Epworth Sleepiness Scale dargestellt und mit den Werten der Gruppen der Schlafpatienten und der Kontrollpersonen von Bloch et al. verglichen (Bloch et al. 1999). 
Tabelle 6: Ergebnisse der ESS im Vergleich mit den Werten von Bloch et al.

\begin{tabular}{|c|c|c|c|c|c|}
\hline & $\begin{array}{l}\text { männl. } \\
\text { Patienten } \\
\mathrm{n}=28\end{array}$ & $\begin{array}{l}\text { weibl. } \\
\text { Patienten } \\
n=19\end{array}$ & $\begin{array}{l}\text { alle } \\
n=47\end{array}$ & $\begin{array}{l}\text { Bloch } \\
\text { Patienten } \\
n=174\end{array}$ & $\begin{array}{l}\text { Bloch } \\
\text { Kontrollen } \\
n=159\end{array}$ \\
\hline \multicolumn{6}{|l|}{ ESS } \\
\hline Minimum & 3 & 1 & 1 & & \\
\hline Maximum & 22 & 18 & 22 & & \\
\hline Mittelwert & 10,46 & 8,47 & 9,66 & 13,0 & 5,7 \\
\hline Std.abw. & 4,45 & 4,58 & 4,56 & 5,1 & 3,0 \\
\hline
\end{tabular}

Für die Auswertung der ESS konnten nur 19 der 22 weiblichen Patienten berücksichtigt werden, da die restlichen Patientinnen in dem Fragebogen mehr als eine Frage nicht beantwortet hatten. Somit konnten diese Antworten nicht gewertet werden.

Der Mittelwert der männlichen Patienten sowie deren Minimal- und Maximalwerte liegen über den entsprechenden Werten der Patientinnen. Dieser Unterschied ist statistisch allerdings nicht signifikant. Die Abbildung 8 veranschaulicht diese Ergebnisse.

Im Vergleich mit den Referenzgruppen von Bloch et al. liegt der durchschnittliche Wert für Tagesschläfrigkeit bei den Parkinsonpatienten insgesamt über dem Mittelwert der Kontrollgruppe, jedoch unter dem des Patientenkollektivs mit klinisch relevanten Schlafproblemen.

\subsubsection{Pittsburgh Sleep Quality Index (PSQI)}

Im Folgenden werden die Ergebnisse des PSQI-Gesamtwertes der Patientengruppe dargestellt und mit Hilfe des Grenzwertes zur Differenzierung zwischen ,guten“ und „schlechten“ Schläfern beschrieben (gute Schläfer $\leq 5$ Punkte; schlechte Schläfer $>5$ Punkte).

Eine detaillierte Darstellung der Werte der einzelnen Rubriken des Pittsburgh Sleep Quality Index’ findet sich in Abbildung 9 im Anhang.

Tabelle 7: Ergebnisse des PSQI-Gesamtwertes

\begin{tabular}{|l|l|l|l|}
\hline & $\begin{array}{l}\text { männliche } \\
\text { Patienten } \\
\mathrm{n}=28\end{array}$ & $\begin{array}{l}\text { weibliche } \\
\text { Patienten } \\
\mathrm{n}=22\end{array}$ & alle \\
$\mathrm{n}=50$
\end{tabular}

Der PSQI-Mittelwert der Patientinnen liegt im Vergleich zu dem der männlichen Probanden bei in etwa vergleichbarer Standardabweichung um mehr als einen Punkt höher. Hierbei handelt es sich 
allerdings nicht um einen statistisch signifikanten Unterschied der Schlafqualität zwischen männlichen und weiblichen Patienten.

Insgesamt geben 26 der 50 teilnehmenden Patienten einen jenseits des Grenzwertes für eine gute Schlafqualität liegenden Wert an. Demnach befinden sich unter den weiblichen Patienten 59,1 Prozent „schlechte“ Schläfer; bei den Männern betrifft dies 46,4 Prozent (Abbildung 10).

\subsection{Ergebnisse der Betreuergruppe}

Im Folgenden werden die Ergebnisse der Anamnese und Fragebögen dargestellt und mit Referenzwerten der Originalstudien bzw. Ergebnissen zweier Vorstudien (Linkersdörfer 2006; Stiens 2000) verglichen. Da sich teilweise für betreuende Söhne und Töchter unterschiedlich signifikante Ergebnisse zeigten, erfolgt die Darstellung in diesen Fällen getrennt nach Geschlechtern. Darüber hinaus werden Unterschiede in Bezug auf hauptbetreuende und nicht-hauptbetreuende Angehörige untersucht.

\subsubsection{Anamnese}

Die Gruppe der betreuenden Kinder (fortan Betreuer) bestand aus 19 Söhnen (38\%) und 31 Töchtern (62\%) der Parkinsonpatienten. Die Söhne kümmerten sich in 68\% der Fälle um den Vater und in 32\% um die Mutter. Die Töchter betreuten zu 48\% den Vater und zu 52\% die Mutter.

Die betreuenden erwachsenen Kinder waren zwischen 22 und 58 Jahre alt. Das Durchschnittsalter lag insgesamt bei 38,1 Jahren ( $\mathrm{SD}=9,5)$, wobei die Söhne mit einem mittleren Alter von 35,6 Jahren $(\mathrm{SD}=7,5)$ im Vergleich zu den betreuenden Töchtern mit einem mittleren Alter von 39,7 Jahren etwas jünger waren $(\mathrm{SD}=10,4)$. Vierzig Prozent der Betreuer waren ledig, 52\% verheiratet und 6\% geschieden. Eine betreuende Tochter war verwitwet. Fast die Hälfte der Betreuer (48\%) hatte keine eigenen Kinder, $20 \%$ hatten ein Kind, 28\% zwei Kinder und zwei betreuende Kinder hatten drei oder mehr eigene Kinder. Zehn Betreuer lebten zum Zeitpunkt der Befragung alleine, 38 mit einem Partner und zwei mit Angehörigen zusammen. Achtundsiebzig Prozent zählten sich der Stadtbevölkerung, $22 \%$ der Landbevölkerung zu.

Bezüglich der beruflichen Situation gliederte sich die Betreuergruppe folgendermaßen: 12\% Hausfrauen/-männer, 46\% der betreuenden Kinder gehörten zur Gruppe der Arbeiter, einfachen Angestellten und Beamten im einfachen/mittleren Dienst, 26\% zur Gruppe der leitenden Angestellten, Beamten im gehobenen/höheren Dienst und Selbständigen, 4\% Frührentnerinnen und 12\% Studenten. Lediglich ein Drittel der an der Untersuchung teilnehmenden Kinder gab an, Hauptbetreuer ihres erkrankten Elternteils zu sein. In vier Fällen handelte es sich hierbei um den betreuenden Sohn, in 13 Fällen um die betreuende Tochter. Die restlichen zwei Drittel der Kinder gaben den nicht-erkrankten Elternteil bzw. den erkrankten Elternteil selbst als hauptsächlich verantwortliche Person an. Allgemein 
erhielten die haupt- sowie nicht-hauptbetreuenden Kinder in $64 \%$ Unterstützung durch ihr nichterkranktes Elternteil, in $10 \%$ von den Geschwistern und in 6\% vom eigenen Partner. In einem Fall wurde die Unterstützung durch die eigenen Kinder, in einem weiteren Fall durch ein Pflege-/ Wohnheim und in zwei Fällen durch andere Personen geleistet. Sechs Betreuer gaben an, keine Unterstützung von Dritten zu bekommen und damit in der Betreuung des erkrankten Elternteils auf sich alleine gestellt zu sein.

22 Betreuer hatten mehr als zwanzigmal pro Monat persönlich oder via Telefon Kontakt zu ihrem erkrankten Elternteil, drei Betreuer 16-20 mal, sieben 11-15 mal, zehn 6-10 mal, und acht Betreuer hatten ein bis fünfmal pro Monat Kontakt zum erkrankten Elternteil. Gut die Hälfte der betreuenden Kinder (52\%) wohnte in relativ großer räumlicher Nähe zum erkrankten Elternteil (Entfernung 0-5 $\mathrm{km})$. Von ihnen lebten je ein Sohn und eine Tochter mit ihrem erkrankten Elternteil in einer gemeinsamen Wohnung, sowie fünf Töchter und ein Sohn mit ihrem erkrankten Elternteil in einem Haus mit getrennten Wohnungen. Sechs Prozent lebten 6-10 km entfernt, zwei Prozent bis zu dreißig Kilometer und vier Prozent 51-100 km entfernt. Die verbleibenden 31\% der betreuenden Kinder lebten in einer Entfernung von über $100 \mathrm{~km}$ zu ihrem jeweiligen erkrankten Elternteil.

Bezüglich der eigenen Gesundheit gaben zum Zeitpunkt der Datenerhebung zwei Drittel der Betreuer keine Erkrankungen an. Elf Betreuer litten an einer, drei an zwei, ein betreuendes Kind an vier und zwei an fünf Erkrankungen. In 76\% der Fälle wurde kein Medikament eingenommen, in 16\% ein Medikament und in $8 \%$ vier oder mehr verschiedene Medikamente.

\subsubsection{Filial Anxiety Scale (FAS)}

Die Mittelwerte und Standardabweichungen der FAS sind in Tabelle 8 im Vergleich mit den Werten von Cicirellis Referenzgruppe dargestellt (Cicirelli 1988).

Tabelle 8: Mittelwerte und Standardabweichungen der Filial Anxiety Scale im Vergleich mit den Ergebnissen von Cicirelli (Cicirelli 1988)

\begin{tabular}{|l|r|r|r|r|}
\hline & $\begin{array}{l}\text { Söhne } \\
\mathrm{n}=19\end{array}$ & $\begin{array}{l}\text { Töchter } \\
\mathrm{n}=31\end{array}$ & $\begin{array}{l}\text { alle } \\
\mathrm{n}=50\end{array}$ & $\begin{array}{l}\text { Cicirelli } \\
\mathrm{n}=71\end{array}$ \\
\hline Filial Anxiety Scale A & 7 & 7 & 7 & \\
\hline Minimum & 29 & 30 & 30 & \\
\hline Maximum & 17,68 & 16,94 & 17,22 & 16,8 \\
\hline Mittelwert & 7,07 & 6,77 & 6,82 & 4,9 \\
\hline Standardabweichung & & & & \\
\hline Filial Anxiety Scale B & 8 & 12 & 8 & \\
\hline Minimum & 27 & 30 & 30 & 20,0 \\
\hline Maximum & 19,16 & 21,83 & 20,81 & 4,2 \\
\hline Mittelwert & 5,32 & 4,54 & 4,97 & \\
\hline Standardabweichung & & & & \\
\hline
\end{tabular}

Die Ergebnisse zeigen für die betreuenden Söhne im Vergleich zu den Töchtern einen höheren Mittelwert in der FAA. In der FAB liegt hingegen der Mittelwert der betreuenden Töchter um mehr 
als zwei Punkte höher als bei den Söhnen. Die Söhne sorgen sich im Vergleich zu den Töchtern demnach mehr über ihre eigenen Fähigkeiten, den Anforderungen der Betreuungssituation gerecht werden zu können. Die Sorge um das Wohlergehen der alternden Eltern ist wiederum bei den betreuenden Töchtern ausgeprägter. Die Analyse ergibt jedoch keine signifikanten geschlechtsspezifischen Unterschiede. Der beschriebene Fund ist daher lediglich als Trend zu werten.

Auffällig ist, dass der Mittelwert der FAB für beide Geschlechter über dem der FAA liegt, obwohl der maximal zu erreichende Gesamtwert der FAB bei 30 Punkten und damit unter dem höchstmöglichen Wert der FAA (35 Punkte) liegt. Dieses Phänomen kann in Cicirellis Stichprobe ebenfalls beobachtet werden.

Hinsichtlich einer Differenzierung der Ergebnisse nach dem Status des Haupt- bzw. NichtHauptbetreuers ergeben sich für die hauptbetreuenden Angehörigen durchschnittlich höhere Werte für die FAA und FAB als für die Nicht-Hauptbetreuer. Dabei sind auf einem Niveau von $p=0,021$ statistisch signifikante Unterschiede in der FAB festzustellen.

Eine graphische Darstellung einiger dieser Ergebnisse findet sich in den Abbildungen 11-14.

Im Vergleich mit der Referenzgruppe von Cicirelli zeigen die betreuenden Angehörigen lediglich minimal höhere Mittelwerte für beide Unterskalen, die innerhalb der Standardabweichungen von Cicirelli liegen.

\subsubsection{Louvain Filial Maturity Scale A (LFMS-A)}

Die Mittelwerte und Standardabweichungen des Filiale-Reife-Bogens sind in Tabelle 9 im Vergleich mit den Werten zweier Vorstudien (Stiens 2000, Linkersdörfer 2006) dargestellt.

Tabelle 9: Mittelwerte und Standardabweichungen des Filiale-Reife-Bogens (LFMS-A) im Vergleich mit den Ergebnissen zweier Vorstudien (Stiens 2000 [G.S.], Linkersdörfer 2006 [V.L.])

\begin{tabular}{|l|r|r|r|r|r|}
\hline & \multicolumn{1}{l|}{$\begin{array}{l}\text { Söhne } \\
\mathrm{n}=19\end{array}$} & \multicolumn{1}{l|}{$\begin{array}{l}\text { Töchter } \\
\mathrm{n}=31\end{array}$} & \multicolumn{1}{l|}{$\begin{array}{l}\text { alle } \\
\mathrm{n}=50\end{array}$} & \multicolumn{1}{l|}{$\begin{array}{l}\text { G.S. 2000 } \\
\mathrm{n}=61\end{array}$} & \multicolumn{2}{l|}{$\begin{array}{l}\text { V.L. 2006 } \\
\mathrm{n}=47\end{array}$} \\
\hline filiale Liebe & 5,04 & 5,42 & 5,28 & 4,53 & 4,60 \\
& $(0,81)$ & $(0,92)$ & $(0,89)$ & $(0,99)$ & $(0,68)$ \\
\hline fil. Verpflicht. & 4,16 & 5,06 & 4,72 & 5,29 & 5,35 \\
& $(1,16)$ & $(1,22)$ & $(1,27)$ & $(0,94)$ & $(0,77)$ \\
\hline fil. Hilfsbereit. & 4,65 & 5,38 & 5,10 & 5,23 & 5,26 \\
& $(0,93)$ & $(0,86)$ & $(0,95)$ & $(0,77)$ & $(0,83)$ \\
\hline filiale Hilfe & 4,55 & 5,63 & 5,22 & 5,98 & 5,82 \\
& $(1,15)$ & $(0,93)$ & $(1,14)$ & $(0,63)$ & $(0,71)$ \\
\hline fil. Autonom. & 4,08 & 3,87 & 3,95 & 4,31 & 2,90 \\
& $(0,73)$ & $(0,68)$ & $(0,70)$ & $(0,66)$ & $(0,60)$ \\
\hline elt. Besorgnis & 5,33 & 5,24 & 5,28 & 4,45 & 4,29 \\
& $(0,66)$ & $(0,95)$ & $(0,84)$ & $(0,88)$ & $(0,79)$ \\
\hline fam. Solidar. & 4,03 & 4,56 & 4,36 & 4,55 & 4,46 \\
& $(1,03)$ & $(1,10)$ & $(1,10)$ & $(0,68)$ & $(0,91)$ \\
\hline
\end{tabular}

Während die „filiale Liebe“ der Töchter im Vergleich zu den männlichen Betreuern nur geringfügig höher ausfällt, weisen die Töchter in den Rubriken „filiales Verpflichtungsgefühl“ (FO), „filiale 
Hilfsbereitschaft" (FHN) und „filiale Hilfe“ (FH) höhere Durchschnittswerte auf, die sich auf einem Signifikanzniveau von $\mathrm{p}<0,05$ von den entsprechenden Ergebnissen der betreuenden Söhne unterscheiden (FO: p=0,004; FHN: p=0,002; FH: p=0,001). Demgegenüber zeigten betreuende Söhne eine vergleichsweise größere „filiale Autonomie“ und schätzten auch die „elterliche Besorgnis“ als höher ein. Die „familiäre Solidarität“ wurde wiederum von den Töchtern als größer empfunden. Die Abbildungen 15-17 veranschaulichen diese Ergebnisse.

Die hauptbetreuenden Angehörigen zeigen im Vergleich mit den Nicht-Hauptbetreuern durchschnittlich höhere Werte für „filiale Liebe“, „filiales Verpflichtungsgefühl“, „filiale Hilfsbereitschaft“, „filiale Hilfe“ und „familiäre Solidarität". Die nicht-hauptbetreuenden Söhne und Töchter weisen hingegen höhere Werte für „filiale Autonomi““ und „elterliche Besorgnis“ auf (Abbildungen 18 und 19). Signifikante Unterschiede wurden diesbezüglich jedoch durch den Wilcoxon-Mann-Whitney-U-Test auf einem Niveau von $\mathrm{p}<0,05$ ausgeschlossen.

Im Vergleich zu den Ergebnissen der Vorstudien zeigen sowohl die betreuenden Töchter als auch Söhne durchschnittlich höhere Werte für „filiale Liebe“ und „elterliche Besorgnis“ als die Probanden der Untersuchungen von Stiens und Linkersdörfer (Stiens 2000, Linkersdörfer 2006). Die Scores für „filiales Verpflichtungsgefühl“, „filiale Hilfsbereitschaft“ und „familiäre Solidarität“ fallen zwar niedriger aus, liegen jedoch innerhalb der Standardabweichungen der Vergleichsstudien. Die Werte für „filiale Hilfe“ erweisen sich ebenfalls als niedriger, liegen hingegen außerhalb der Standardabweichungen der Untersuchungen von Stiens. Die filiale Autonomie der betreuenden Söhne und Töchter der aktuellen Studie liegt mit einem Mittelwert von 3,95 zwischen den beiden Vergleichswerten und außerhalb der Standardabweichung von Linkersdörfer.

\subsubsection{Fragebogen zur Belastung durch die Betreuung}

Die Ergebnisse des Belastungsbogens sind in Tabelle 10 im Vergleich mit den Werten einer Vorstudie an pflegenden Angehörigen Demenzkranker (Stiens 2000) dargestellt.

Tabelle 10: Extremwerte, Mittelwerte und Standardabweichungen des Belastungsbogens im Vergleich mit den Ergebnissen der Vorstudie von Stiens (Stiens 2000 [G.S.])

\begin{tabular}{|l|l|l|l|r|}
\hline & $\begin{array}{l}\text { Söhne } \\
\mathrm{n}=19\end{array}$ & $\begin{array}{l}\text { Töchter } \\
\mathrm{n}=30\end{array}$ & $\begin{array}{l}\text { alle } \\
\mathrm{n}=49\end{array}$ & $\begin{array}{l}\text { G.S. 2000 } \\
\mathrm{n}=61\end{array}$ \\
\hline Belastungsbogen & & & & 7 \\
\hline Minimum & 0 & 5 & 0 & 47 \\
\hline Maximum & 28 & 35 & 35 & 24,41 \\
\hline Mittelwert & 12,47 & 15,10 & 14,08 & 8,79 \\
\hline Standardabweichung & 7,42 & 6,70 & 7,03 & \\
\hline
\end{tabular}

Aus den in der Tabelle 10 dargestellten Ergebnissen ist ersichtlich, dass sich die Töchter im Allgemeinen durch die Betreuung des erkrankten Elternteils stärker belastet fühlen als die Söhne. 
Dafür sprechen sowohl der vergleichsweise höhere Mittelwert, als auch die höheren Minimal- und Maximalwerte der betreuenden Töchter.

Bezüglich der Differenzierung der Ergebnisse hinsichtlich des Status des Haupt- bzw. NichtHauptbetreuers ergeben sich auf einem Niveau von $p<0,05$ signifikante Unterschiede $(p=0,034)$. Der Mittelwert der hauptbetreuenden Söhne und Töchter liegt bei 17,18 (Standardabweichung 7,55), während das mittlere Belastungserleben der nicht-hauptbetreuenden Angehörigen bei einem Wert von 12,44 liegt (Standardabweichung 6,25). Abbildung 20 stellt die Ergebnisse graphisch dar.

Im Vergleich mit den Ergebnissen der Vorstudie (Stiens 2000) zeigen sich in der aktuellen Untersuchung ein geringerer Mittelwert, sowie niedrigere Minimal- und Maximalwerte.

\subsubsection{Short-Form-36 Health Survey (SF-36)}

Tabelle 11 bildet die Ergebnisse des Fragebogens zur Lebensqualität (SF-36) im Vergleich mit dem Bundes-Gesundheitssurvey von 1998 ab (Ellert und Bellach 1999). Diese vom Robert Koch-Institut Berlin durchgeführte Erhebung stellt eine repräsentative Normstichprobe für die Bundesrepublik Deutschland dar. Als Referenz für die Ergebnisse der betreuenden Söhne dient aufgrund ihres mittleren Alters von 35,6 Jahren die Gruppe der Männer zwischen 30 und 39 Jahren. Bei den betreuenden Töchtern wurde zum Vergleich die Altersgruppe der Frauen von 40 bis 49 Jahren gewählt, da das mittlere Alter der an der Untersuchung teilnehmenden Töchter 39,7 Jahre betrug und der Median bei 42 Jahren lag.

Tabelle 11: Mittelwerte und Standardabweichungen des SF-36 im Vergleich mit der Normstichprobe des Bundes-Gesundheitssurveys 1998 (Ellert und Bellach 1999)

\begin{tabular}{|l|r|r|r|r|r|}
\hline & \multicolumn{1}{l|l}{ Söhne } & Töchter & alle & $\begin{array}{l}\text { Männer } \\
\text { Normstichprobe }\end{array}$ & $\begin{array}{l}\text { Frauen } \\
\text { Normstichprobe } \\
\text { 30-39 J. }\end{array}$ \\
& $\mathrm{n}=19$ & $\mathrm{n}=31$ & $\mathrm{n}=50$ J. \\
\hline körperliche & 93,42 & 92,90 & 93,10 & 94,31 & 87,29 \\
Funktionsfähigkeit & $(14,50)$ & $(13,71)$ & $(13,70)$ & $(11,64)$ & $(17,14)$ \\
\hline körperliche & 96,05 & 80,65 & 86,50 & 93,18 & 82,58 \\
Rollenfunktion & $(12,54)$ & $(30,08)$ & $(25,86)$ & $(20,14)$ & $(31,21)$ \\
\hline körperliche & 88,84 & 84,16 & 85,94 & 76,15 & 63,69 \\
Schmerzen & $(15,55)$ & $(23,69)$ & $(20,92)$ & $(22,41)$ & $(24,23)$ \\
\hline allgemeine & 69,00 & 72,19 & 70,98 & 70,74 & 66,40 \\
Gesundheit & $(21,07)$ & $(20,74)$ & $(20,71)$ & $(16,21)$ & $(18,30)$ \\
\hline & 67,11 & 59,03 & 62,10 & 62,56 & 57,44 \\
Vitalität & $(12,06)$ & $(19,12)$ & $(17,12)$ & $(15,95)$ & $(18,81)$ \\
\hline soziale & 87,50 & 85,08 & 86,00 & 89,98 & 84,17 \\
Funktionsfähigkeit & $(16,67)$ & $(17,80)$ & $(17,25)$ & $(17,34)$ & $(20,67)$ \\
\hline emotionale & 89,47 & 89,25 & 89,33 & 94,26 & 86,93 \\
Rollenfunktion & $(22,37)$ & $(21,76)$ & $(21,76)$ & $(17,67)$ & $(28,30)$ \\
\hline psychisches & 76,00 & 75,48 & 75,68 & 75,56 & 69,47 \\
Wohlbefinden & $(13,79)$ & $(13,38)$ & $(13,340)$ & $(14,49)$ & $(17,73)$ \\
\hline
\end{tabular}

Im Großen und Ganzen sind die Ergebnisse der Gruppen der männlichen und weiblichen Betreuer in etwa miteinander vergleichbar. Lediglich in den Rubriken körperliche Rollenfunktion und Vitalität 
liegen die Mittelwerte der betreuenden Söhne mit >15 bzw. > 8 Prozentpunkten eindeutig über denen der Töchter. Eine statistische Signifikanz auf einem Niveau von $p<0,05$ lässt sich jedoch nicht feststellen. Die Analyse bezüglich des Hauptbetreuer-Status ergibt ebenfalls keine signifikanten Unterschiede in der gesundheitsbezogenen Lebensqualität. Die Abbildungen 21 und 22 veranschaulichen die Ergebnisse des SF-36 der Betreuergruppe.

Im Vergleich mit der entsprechenden Referenzgruppe des Bundes-Gesundheitssurveys geben die betreuenden Söhne weniger körperliche Schmerzen und eine bessere Vitalität an. Die emotionale Rollenfunktion liegt allerdings fast fünf Prozent unter der der altersbezogenen Referenzgruppe. Die betreuenden Töchter schätzen ihre körperliche Funktionsfähigkeit, ihre allgemeine Gesundheit und ihr psychisches Wohlbefinden besser ein als die altersentsprechenden Probandinnen der Referenzstudie. Der Mittelwert für körperliche Schmerzen liegt in der Gruppe der Töchter vergleichsweise sogar 20\% niedriger.

Die Abweichungen in den Mittelwerten verbleiben jedoch allesamt innerhalb der Standardabweichungen der Referenzgruppe.

\subsubsection{0-Item Center for Epidemiological Studies Depression Scale (CESD-10)}

Im Folgenden werden die Ergebnisse der CESD-10 dargestellt. Der Grenzwert für das Vorhandensein depressiver Symptome liegt bei zehn Punkten $(\geq 10)$.

Tabelle 12: Ergebnisse der CESD-10

\begin{tabular}{|l|r|l|r|}
\hline & $\begin{array}{l}\text { Söhne } \\
\mathrm{n}=19\end{array}$ & $\begin{array}{l}\text { Töchter } \\
\mathrm{n}=31\end{array}$ & $\begin{array}{l}\text { alle } \\
\mathrm{n}=50\end{array}$ \\
\hline CESD-10 & & & 0 \\
\hline Minimum & 0 & 1 & 15 \\
\hline Maximum & 15 & 14 & 5,96 \\
\hline Mittelwert & 5,53 & 6,23 & 3,68 \\
\hline Standardabweichung & 3,47 & 3,84 & 9 \\
\hline Anzahl über cut-off & 1 & 8 & 18 \\
\hline Prozent über cut-off & 5,3 & 25,8 & \\
\hline
\end{tabular}

Die Untersuchung ergab für die betreuenden Söhne mit Einzelwerten zwischen 0 und 15 Punkten einen Mittelwert von 5,53 Punkten in der CESD-10. Der Mittelwert der Töchter lag geringfügig höher. Damit liegen sowohl die geschlechtsspezifischen Mittelwerte als auch der Gesamtmittelwert unter dem von Andresen et al. vorgegebenen cut-off von 10 Punkten für Depressivität. Es liegen demnach bei acht Töchtern und einem der Söhne Hinweise auf eine depressive Störung vor (Abbildung 23). Das sind $18 \%$ der Gesamtstichprobe.

Die Analyse ergab auf einem Niveau von $\mathrm{p}<0,05$ weder bezüglich des Geschlechts des betreuenden Kindes noch bezüglich des Hauptbetreuer-Status' statistisch signifikante Unterschiede. 


\subsubsection{Epworth Sleepiness Scale (ESS)}

In Tabelle 13 werden die Ergebnisse der Epworth Sleepiness Scale dargestellt und mit denen der Schlafpatienten und Kontrollpersonen von Bloch et al. verglichen (Bloch et al. 1999).

Tabelle 13: Ergebnisse der ESS im Vergleich mit den Werten von Bloch et al. (1999)

\begin{tabular}{|c|c|c|c|c|c|}
\hline & $\begin{array}{l}\text { Söhne } \\
\mathrm{n}=19\end{array}$ & $\begin{array}{l}\text { Töchter } \\
n=31\end{array}$ & $\begin{array}{l}\text { alle } \\
\mathrm{n}=50\end{array}$ & $\begin{array}{l}\text { Bloch } \\
\text { Patienten } \\
\mathrm{n}=174\end{array}$ & $\begin{array}{l}\text { Bloch } \\
\text { Kontrolle } \\
\text { n = } 159\end{array}$ \\
\hline \multicolumn{6}{|l|}{ ESS } \\
\hline Minimum & 0 & 1 & 0 & & \\
\hline Maximum & 13 & 18 & 18 & & \\
\hline Mittelwert & 5,37 & 7,13 & 6,46 & 13,0 & 5,7 \\
\hline Standardabweichung & 3,66 & 3,56 & 3,66 & 5,1 & 3,0 \\
\hline
\end{tabular}

Die betreuenden Töchter zeigen einen insgesamt höheren Mittelwert der ESS als die betreuenden Söhne. Eine statistische Signifikanz dieser Unterschiede wird auf einem Niveau von $p<0,05$ allerdings verfehlt. Die Analyse ergibt ebenfalls keinen signifikanten Unterschied bezüglich hauptbetreuenden und nicht-hauptbetreuenden Angehörigen. Die Abbildungen 24 und 25 veranschaulichen diese Ergebnisse.

Im Vergleich mit den Schlafpatienten der Studie von Bloch und Mitarbeitern zeigen die Probanden der aktuellen Untersuchung deutlich niedrigere ESS-Werte und damit eine allgemein geringere Tagesschläfrigkeit. Die Ergebnisse der betreuenden Söhne entsprechen in etwa denen der Kontrollgruppe von Bloch et al. Die Töchter weisen im Mittel eine etwas höhere Tagesschläfrigkeit auf als die Kontrollpersonen in der Originalstudie. Auch bei Bloch und Mitarbeitern blieben die geschlechtsspezifischen Unterschiede nicht signifikant.

\subsubsection{Pittsburgh Sleep Quality Index (PSQI)}

Im Folgenden werden die Ergebnisse des Gesamtwertes des PSQI dargestellt und mit den Werten von Zeitlhofer et al. verglichen (Zeitlhofer et al. 2000). Der Grenzwert zur Unterscheidung zwischen „guten“ und „schlechten“ Schläfern liegt bei 5 Punkten (gute Schläfer $\leq 5$; schlechte Schläfer $>5$ ). Eine detaillierte tabellarische Darstellung der Ergebnisse der sieben PSQI-Unterscores ist im Anhang in Abbildung $26 \mathrm{zu}$ finden. 
Tabelle 14: Ergebnisse des PSQI im Vergleich mit den Werten von Zeitlhofer et al. (2000)

\begin{tabular}{|l|r|r|r|r|}
\hline & $\begin{array}{l}\text { Söhne } \\
\mathrm{n}=19\end{array}$ & $\begin{array}{l}\text { Töchter } \\
\mathrm{n}=31\end{array}$ & $\begin{array}{l}\text { alle } \\
\mathrm{n}=50\end{array}$ & $\begin{array}{l}\text { Zeitlhofer } \\
\mathrm{n}=991\end{array}$ \\
\hline PSQI gesamt & & 0 & 0 & \\
\hline Minimum & 1 & 13 & 13 & 4,55 \\
\hline Maximum & 10 & 5,23 & 4,92 & 3,71 \\
\hline Mittelwert & 4,42 & 3,11 & 2,73 & 318 \\
\hline Standardabweichung & 1,95 & 11 & 15 & 32,1 \\
\hline Anzahl über cut-off & 4 & 35,5 & 30,0 & \\
\hline Prozent über cut-off & 21,1 & & & \\
\hline
\end{tabular}

Der Mittelwert der betreuenden Töchter liegt etwas über dem durchschnittlichen PSQI-Gesamtwert der männlichen Betreuer. Die Streubreite der Einzelwerte ist bei den Töchtern im Vergleich ebenfalls höher. Während von den betreuenden Söhnen lediglich 21,1\% über dem Grenzwert liegen, gilt dies bei den Töchtern für 35,5 Prozent (Abbildung 27).

Weder bezüglich des Geschlechts des betreuenden Kindes noch bezüglich des Hauptbetreuer-Status' können in der Analyse auf einem Niveau von $p<0,05$ statistisch signifikante Unterschiede in der Schlafqualität gezeigt werden.

Im Vergleich mit der Referenzgruppe von Zeitlhofer und Mitarbeitern zeigen die betreuenden Angehörigen insgesamt einen nur geringfügig höheren Mittelwert. Der Prozentsatz über dem Grenzwert von 5 Punkten ist für beide Gruppen vergleichbar.

\subsection{Ergebnisse der Kontrollgruppe}

Im Folgenden werden die Ergebnisse der Anamnese und Fragebögen der Kontrollgruppe dargestellt und mit den Werten der betreuenden Söhne und Töchter, die an dieser Untersuchung teilgenommen haben, verglichen. Da sich dabei teilweise geschlechtsspezifische Unterschiede zeigten, erfolgt die Darstellung in diesen Fällen getrennt nach Geschlechtern.

\subsubsection{Anamnese}

Entsprechend den Vorgaben aus der Gruppe der betreuenden Angehörigen umfasst die Gruppe der Kontrollpersonen 19 Söhne und 31 Töchter von nicht chronisch kranken oder anderweitig pflegebedürftigen Eltern. Die Alterspanne der teilnehmenden Kontrollpersonen reichte von 22 bis 56 Jahre. Das Durchschnittsalter lag bei 38 Jahren (Männer 35,7 ( $\mathrm{SD}=7,8$ ) und Frauen 39,4 Jahre $(\mathrm{SD}=10,4)) .36 \%$ gaben an, ledig zu sein, 58\% waren verheiratet und acht Prozent der Kontrollpersonen geschieden. 22 Studienteilnehmer hatten keine eigenen Kinder, zehn hatten ein Kind, weitere zehn zwei Kinder, und acht Personen hatten drei oder mehr Kinder. Damit liegt die Anzahl eigener Kinder in der Kontrollgruppe etwas höher als bei den Betreuern. Elf Kontrollpersonen 
lebten alleine, 37 mit einem Partner und zwei mit Angehörigen zusammen. 62\% lebten in der Stadt, $38 \%$ auf dem Land.

Ebenfalls übereinstimmend mit den betreuenden Söhnen und Töchtern gehörten auch $46 \%$ der Kontrollpersonen der Gruppe der Arbeiter, einfachen Angestellten und Beamten im einfachen/mittleren Dienst an und 26\% der Gruppe der leitenden Angestellten, Beamten im gehobenen/höheren Dienst und Selbständigen. Sechzehn Prozent waren Hausfrauen/-männer und zwölf Prozent Studenten.

Bei 35 Kontrollpersonen lebten noch beide Elternteile, in zwölf Fällen war der Vater bereits verstorben, in drei Fällen die Mutter. Das väterliche Durchschnittsalter lag bei 66,1 Jahren $(S D=9,6)$, das mittlere Alter der Mütter bei 64,9 Jahren (SD=10,4). Damit waren die Eltern der Kontrollpersonen durchschnittlich etwas jünger als die in die Studie eingeschlossenen Patienten.

$26 \%$ der Kontrollpersonen hatten über zwanzigmal pro Monat Kontakt mit ihren Eltern, zwei Prozent 16-20 mal, zwanzig Prozent 11-15 mal, 22 Prozent 6-10 mal und 30 Prozent ein bis fünfmal pro Monat. Die räumliche Distanz zu den Eltern betrug in elf Fällen 0-5 km, in je fünf Fällen 6-10 bzw. 11-30 km und in drei Fällen 31-50 km. Vier Kontrollpersonen wohnten zwischen 51 und 100 km von ihren Eltern entfernt und 22 über hundert Kilometer. Im Vergleich zu den betreuenden Söhnen und Töchtern stand die Kontrollgruppe somit weniger häufig pro Monat im Kontakt mit ihren Eltern und wohnte durchschnittlich auch weiter entfernt.

60\% der Kontrollgruppe gab an, zum Untersuchungszeitpunkt unter keiner Erkrankung zu leiden. 32\% hatten eine, vier Prozent zwei und ebenfalls vier Prozent vier Erkrankungen. Die durchschnittliche Anzahl der Erkrankungen pro Kopf lag in der Kontrollgruppe bei 0,56, die durchschnittliche Anzahl eingenommener Medikamente bei 0,38 (zum Vergleich: mittlere Anzahl Erkrankungen Betreuer 0,62; mittlere Anzahl Medikamente Betreuer 0,48).

\subsubsection{Mini-Mental Status-Test (MMST)}

Der Mini-Mental Status-Test wurde vor Beginn des Interviews bei denjenigen Kontrollpersonen durchgeführt, bei denen ein persönliches Gespräch zur Studienteilnahme stattfand. Da der MMST unter anderem auch praktische Aufgaben beinhaltet, war die Durchführung des Tests bei den Probanden, die die Fragebögen per Post zugeschickt bekamen, nicht möglich.

Von der Gruppe der Kontrollpersonen nahmen daher lediglich 6 von 50 Probanden an dem Test teil. Die dabei erzielten Ergebnisse lagen zwischen 28 und 30 Punkten. Mittelwert und Median betrugen jeweils 29 Punkte (Standardabweichung 1,10). Die Ergebnisse sind mit denen der Untersuchungsgruppe vergleichbar. 


\subsubsection{Filial Anxiety Scale (FAS)}

In Tabelle 15 werden die Ergebnisse der Filial Anxiety Scale A und B der Studien- und Kontrollgruppe miteinander verglichen.

Tabelle 15: Mittelwerte und Standardabweichungen der Filial Anxiety Scale der Kontrollpersonen im Vergleich mit den Ergebnissen der Studiengruppe

\begin{tabular}{|c|c|c|c|c|c|c|}
\hline & \multicolumn{3}{|c|}{ Studiengruppe } & \multicolumn{3}{|c|}{ Kontrollpersonen } \\
\hline & $\begin{array}{l}\text { Söhne } \\
\mathrm{n}=19\end{array}$ & $\begin{array}{l}\text { Töchter } \\
\mathrm{n}=31\end{array}$ & $\begin{array}{l}\text { alle } \\
\mathrm{n}=50\end{array}$ & $\begin{array}{l}\text { Söhne } \\
\mathrm{n}=19\end{array}$ & $\begin{array}{l}\text { Töchter } \\
\mathrm{n}=31\end{array}$ & $\begin{array}{l}\text { alle } \\
\mathrm{n}=50\end{array}$ \\
\hline \multicolumn{7}{|l|}{ FAA } \\
\hline Minimum & 7 & 7 & 7 & 7 & 7 & 7 \\
\hline Maximum & 29 & 30 & 30 & 23 & 26 & 26 \\
\hline Mittelwert & 17,68 & 16,94 & 17,22 & 13,47 & 15,97 & 15,02 \\
\hline Std.abw. & 7,07 & 6,77 & 6,82 & 3,55 & 5,69 & 5,09 \\
\hline \multicolumn{7}{|l|}{ FAB } \\
\hline Minimum & 8 & 12 & 8 & 11 & 11 & 11 \\
\hline Maximum & 27 & 30 & 30 & 23 & 29 & 29 \\
\hline Mittelwert & 19,16 & 21,83 & 20,81 & 17,84 & 19,45 & 18,84 \\
\hline Std.abw. & 5,32 & 4,54 & 4,97 & 3,27 & 3,89 & 3,72 \\
\hline
\end{tabular}

Die Aufstellung zeigt, dass die Mittelwerte für die Filial Anxiety Scale A und B in der Kontrollgruppe bei insgesamt geringeren Standardabweichungen und Streubreiten der Einzelwerte unter den entsprechenden Ergebnissen der betreuenden Angehörigen liegen. Der Wilcoxon-Mann-Whitney-UTest ergibt für die FAB der Gesamtstichprobe mit $\mathrm{p}=0,01$ einen statistisch signifikanten Unterschied.

Im Gegensatz zu der Studiengruppe, in der die Söhne einen höheren FAA-Wert erreichen als die Töchter, liegt in der Gruppe der Kontrollpersonen sowohl der mittlere FAA- als auch FAB-Wert der weiblichen Probanden über denen der männlichen. Der Wilcoxon-Mann-Whitney-U-Test ergibt für die FAA im Vergleich zwischen betreuenden Söhnen und männlichen Kontrollpersonen und für die FAB im Vergleich zwischen betreuenden Töchtern und weiblichen Probanden der Kontrollgruppe statistisch signifikante Unterschiede (jeweils $\mathrm{p}=0,04$ ). Die Abbildungen 28 und 29 stellen diese Ergebnisse graphisch dar.

\subsubsection{Louvain Filial Maturity Scale A (LFMS-A)}

Die Ergebnisse des Filiale-Reife-Bogens sind in Tabelle 16 im Vergleich mit den entsprechenden Werten der Gruppe der betreuenden Angehörigen dargestellt. 
Tabelle 16: Vergleich der Mittelwerte und Standardabweichungen des Filiale-Reife-Bogens (LFMSA) zwischen der Studien- und Kontrollgruppe

\begin{tabular}{|l|r|r|l|r|r|r|}
\hline & \multicolumn{3}{|l|}{ Studiengruppe } & \multicolumn{2}{l|}{ Kontrollpersonen } \\
\cline { 2 - 8 } & $\begin{array}{l}\text { Söhne } \\
\mathrm{n}=19\end{array}$ & $\begin{array}{l}\text { Töchter } \\
\mathrm{n}=31\end{array}$ & \multicolumn{1}{l|}{$\begin{array}{l}\text { alle } \\
\mathrm{n}=50\end{array}$} & $\begin{array}{l}\text { Söhne } \\
\mathrm{n}=19\end{array}$ & $\begin{array}{l}\text { Töchter } \\
\mathrm{n}=31\end{array}$ & \multicolumn{2}{l|}{$\begin{array}{l}\text { alle } \\
\mathrm{n}=50\end{array}$} \\
\hline filiale Liebe & 5,04 & 5,42 & 5,28 & 5,08 & 5,27 & 5,20 \\
& $(0,81)$ & $(0,92)$ & $(0,89)$ & $(0,49)$ & $(1,02)$ & $(0,86)$ \\
\hline fil. Verpflicht. & 4,16 & 5,06 & 4,72 & 4,77 & 4,98 & 4,90 \\
& $(1,16)$ & $(1,22)$ & $(1,27)$ & $(0,84)$ & $(0,93)$ & $(0,90)$ \\
\hline fil. Hilfsbereit. & 4,65 & 5,38 & 5,10 & 4,79 & 5,10 & 4,99 \\
& $(0,93)$ & $(0,86)$ & $(0,95)$ & $(0,72)$ & $(0,88)$ & $(0,83)$ \\
\hline filiale Hilfe & 4,55 & 5,63 & 5,22 & 4,87 & 5,07 & 4,99 \\
& $(1,15)$ & $(0,93)$ & $(1,14)$ & $(0,82)$ & $(0,98)$ & $(0,92)$ \\
\hline fil. Autonom. & 4,08 & 3,87 & 3,95 & 4,12 & 4,17 & 4,15 \\
& $(0,73)$ & $(0,68)$ & $(0,70)$ & $(0,32)$ & $(0,67)$ & $(0,55)$ \\
\hline elt. Besorgnis & 5,33 & 5,24 & 5,28 & 5,31 & 5,68 & 5,54 \\
& $(0,66)$ & $(0,95)$ & $(0,84)$ & $(0,62)$ & $(0,58)$ & $(0,62)$ \\
\hline fam. Solidar. & 4,03 & 4,56 & 4,36 & 4,77 & 4,87 & 4,83 \\
& $(1,03)$ & $(1,10)$ & $(1,10)$ & $(0,58)$ & $(0,75)$ & $(0,68)$ \\
\hline
\end{tabular}

Die weiblichen Probanden der Kontrollgruppe erreichen in allen sieben Unterskalen des Filiale-ReifeBogens höhere Werte als ihre männlichen Kollegen. Bezüglich der empfundenen „elterlichen Besorgnis“ ergibt die Analyse einen signifikanten Unterschied zwischen den Geschlechtern ( $\mathrm{p}=0,049)$. Bei den Probanden der Kontrollgruppe fallen die Mittelwerte in den Rubriken „filiale Liebe“, „filiale Hilfsbereitschaft" und ,filiale Hilfe“ durchschnittlich niedriger als bei den betreuenden Söhnen und Töchtern aus. Die Werte für „filiales Verpflichtungsgefühl“, „filiale Autonomie“, „elterliche Besorgnis“ und „familiäre Solidarität“ liegen hingegen über den entsprechenden Werten der betreuenden Angehörigen.

Wie aus der Tabelle 16 ersichtlich, liegen bei den männlichen Kontrollpersonen die Mittelwerte für „filiales Verpflichtungsgefühl“, „filiale Hilfsbereitschaft“, „filiale Hilfe“ und empfundene „familiäre Solidarität“ über denen der betreuenden Söhne. Der Wilcoxon-Mann-Whitney-U-Test ergibt auf einem Niveau von $p<0,05$ einen signifikanten Unterschied für die „familiäre Solidarität“ ( $p=0,013)$. Aufgrund von Abweichungen der Mittelwerte voneinander um weniger als 0,1 kann bei diesen Vergleichsgruppen allerdings von vergleichbaren Werten für „filiale Liebe“, „filiale Autonomie“ sowie „elterliche Besorgnis“ ausgegangen werden.

Bei den weiblichen Kontrollpersonen ergeben sich im Vergleich mit der Gruppe der betreuenden Töchter für „filiale Autonomie“, „elterliche Besorgnis“ und „familiäre Solidarität“ höhere Mittelwerte, wohingegen die Werte für „filiale Liebe“, „filiale Hilfsbereitschaft“ sowie „filiale Hilfe“ im Durchschnitt niedriger ausfallen. Für letztere Unterskala ergibt die Analyse einen statistisch signifikanten Unterschied ( $\mathrm{p}=0,022)$.

Insgesamt ist in der Kontrollgruppe die Differenz zwischen den geschlechtsspezifischen Mittelwerten der verschiedenen Unterskalen geringer als die Differenz der jeweiligen Werte bei den betreuenden Söhnen und Töchtern. Die Standardabweichungen in der Gruppe der Kontrollpersonen sind daher 
auch kleiner als bei den Betreuern.

Die Abbildungen 30-33 veranschaulichen einige dieser Ergebnisse graphisch.

\subsubsection{Short-Form-36 Health Survey (SF-36)}

Die Ergebnisse des Fragebogens zur gesundheitsbezogenen Lebensqualität (SF-36) werden im Folgenden dargestellt und mit den entsprechenden Werten der betreuenden Angehörigen verglichen.

Tabelle 17: Mittelwerte und Standardabweichungen des SF-36 der Kontrollgruppe im Vergleich mit den Ergebnissen der Studiengruppe

\begin{tabular}{|l|r|r|l|r|r|r|}
\hline & \multicolumn{3}{|l|}{ Studiengruppe } & \multicolumn{2}{l|}{ Kontrollgruppe } \\
\cline { 2 - 8 } & $\begin{array}{l}\text { Söhne } \\
\mathrm{n}=19\end{array}$ & $\begin{array}{l}\text { Töchter } \\
\mathrm{n}=31\end{array}$ & $\begin{array}{l}\text { llle } \\
\mathrm{n}=50\end{array}$ & $\begin{array}{l}\text { Söhne } \\
\mathrm{n}=19\end{array}$ & $\begin{array}{l}\text { Töchter } \\
\mathrm{n}=31\end{array}$ & $\begin{array}{l}\text { alle } \\
\mathrm{n}=50\end{array}$ \\
\hline $\begin{array}{l}\text { körperl. Funk- } \\
\text { tionsfähigkeit }\end{array}$ & 93,42 \\
$(14,05)$ & 92,90 & 93,10 & 95,00 & 93,23 & 93,90 \\
$(13,71)$ & $(13,70)$ & $(13,94)$ & $(11,30)$ & $(12,26)$ \\
\hline körperl. & 96,05 & 80,65 & 86,50 & 100,00 & 91,94 & 95,00 \\
Rollenfunktion & $(12,54)$ & $(30,08)$ & $(25,86)$ & $(0,00)$ & $(20,80)$ & $(16,75)$ \\
\hline körperl. & 88,84 & 84,16 & 85,94 & 94,32 & 88,84 & 90,92 \\
Schmerzen & $(15,55)$ & $(23,69)$ & $(20,92)$ & $(12,44)$ & $(18,65)$ & $(16,65)$ \\
\hline allgemeine & 69,00 & 72,19 & 70,98 & 76,58 & 76,03 & 76,24 \\
Gesundheit & $(21,07)$ & $(20,74)$ & $(20,71)$ & $(19,08)$ & $(16,41)$ & $(17,28)$ \\
\hline Vitalität & 67,11 & 59,03 & 62,10 & 70,26 & 60,81 & 64,40 \\
& $(12,06)$ & $(19,12)$ & $(17,12)$ & $(12,41)$ & $(16,94)$ & $(15,93)$ \\
\hline soziale Funk- & 87,50 & 85,08 & 86,00 & 95,39 & 91,13 & 92,75 \\
tionsfähigkeit & $(16,67)$ & $(17,80)$ & $(17,25)$ & $(10,38)$ & $(14,86)$ & $(13,39)$ \\
\hline emotionale & 89,47 & 89,25 & 89,33 & 96,49 & 88,17 & 91,34 \\
Rollenfunktion & $(22,37)$ & $(21,76)$ & $(21,76)$ & $(10,50)$ & $(25,17)$ & $(21,09)$ \\
\hline psychisches & 76,00 & 75,48 & 75,68 & 82,32 & 71,10 & 75,36 \\
Wohlbefinden & $(13,79)$ & $(13,38)$ & $(13,40)$ & $(11,10)$ & $(17,06)$ & $(15,93)$ \\
\hline
\end{tabular}

Während die männlichen und weiblichen Kontrollpersonen in den Bereichen „körperliche Funktionsfähigkeit“, „allgemeine Gesundheit“ und „soziale Funktionsfähigkeit“ vergleichbare Mittelwerte erreichen, liegen die Männer in den übrigen Rubriken („körperliche Rollenfunktion“, „körperliche Schmerzen“, „Vitalität“, „emotionale Rollenfunktion“, „psychisches Wohlbefinden“) mehr als fünf Prozentpunkte über den entsprechenden Werten der Frauen (Abbildungen 34 und 35). Aufgrund der Skalenkalibrierung des SF-36 bedeutet letzteres eine in diesen Bereichen bessere Lebensqualität der männlichen Kontrollpersonen (vgl. Kapitel 2.4.7). Bezüglich der „Vitalität“ und dem „psychischen Wohlbefinden“ ergibt die Analyse auf einem Niveau von $p<0,05$ statistisch signifikante Unterschiede (Vital: $\mathrm{p}=0,049$; PsyWo: $\mathrm{p}=0,021$ ).

Der Vergleich zwischen der Betreuer- und Kontrollgruppe zeigt vergleichbare Mittelwerte für „körperliche Funktionsfähigkeit“, „Vitalität“, „emotionale Rollenfunktion“ und „psychisches Wohlbefinden“. In den Rubriken „körperliche Rollenfunktion“ sowie „körperliche Schmerzen“, „allgemeine Gesundheit“ und „soziale Funktionsfähigkeit“ liegen die Mittelwerte der Kontrollpersonen um mehr als fünf Punkte über den entsprechenden Werten der betreuenden Angehörigen. Einen statistisch signifikanten Unterschied ergibt die Analyse bezüglich der „sozialen 
Funktionsfähigkeit“" $(\mathrm{p}=0,034)$.

Die weiblichen Kontrollpersonen erzielen in der „körperlichen Rollenfunktion“ und „sozialen Funktionsfähigkeit“ im Vergleich zu den betreuenden Töchtern deutlich höhere Mittelwerte. Bei den männlichen Kontrollpersonen liegen die durchschnittlichen Werte für „soziale Funktionsfähigkeit“, „emotionale Rollenfunktion“ und „psychisches Wohlbefinden“ über denen der betreuenden Söhne. Darüber hinaus schätzen sie ihre „körperlichen Schmerzen“ als geringer und ihre „allgemeine Gesundheit“ höher ein. Diese Unterschiede verfehlen jedoch allesamt das erforderliche Signifikanzniveau von $\mathrm{p}<0,05$.

\subsubsection{0-Item Center for Epidemiological Studies Depression Scale (CESD-10)}

In Tabelle 18 werden sie Ergebnisse der CESD-10 der Kontrollpersonen dargestellt und mit den Werten der betreuenden Angehörigen verglichen. Der Grenzwert für das Vorliegen einer Depression liegt bei zehn Punkten $(\geq 10)$.

Tabelle 18: Vergleich der Ergebnisse der CESD-10 zwischen der Gruppe der Betreuer und Kontrollpersonen

\begin{tabular}{|l|r|l|l|l|l|r|}
\hline & \multicolumn{4}{|l|}{ Studiengruppe } & \multicolumn{2}{l|}{ Kontrollpersonen } \\
\cline { 2 - 7 } & $\begin{array}{l}\text { Söhne } \\
\mathrm{n}=19\end{array}$ & $\begin{array}{l}\text { Töchter } \\
\mathrm{n}=31\end{array}$ & $\begin{array}{l}\text { alle } \\
\mathrm{n}=50\end{array}$ & $\begin{array}{l}\text { Söhne } \\
\mathrm{n}=19\end{array}$ & $\begin{array}{l}\text { Töchter } \\
\mathrm{n}=31\end{array}$ & $\begin{array}{l}\text { alle } \\
\mathrm{n}=50\end{array}$ \\
\hline CESD-10 & & & & & & 1 \\
\hline Minimum & 0 & 1 & 0 & 0 & 17 & 17 \\
\hline Maximum & 15 & 14 & 15 & 12 & 6,77 & 5,98 \\
\hline Mittelwert & 5,53 & 6,23 & 5,96 & 4,68 & 3,91 & 3,63 \\
\hline Std.abw. & 3,47 & 3,84 & 3,68 & 2,77 & 9 & 10 \\
\hline n über cut-off & 1 & 8 & 9 & 1 & 29,0 & 20,0 \\
\hline \% über cut-off & 5,3 & 25,8 & 18 & 5,3 & &
\end{tabular}

Der Mittelwert der weiblichen Kontrollpersonen liegt im Vergleich zu den männlichen Probanden um mehr als zwei Punkte höher. Die Streubreite der Einzelwerte und die Anzahl der Personen, die einen Score von $\geq 10$ Punkten aufweisen, sind in der Gruppe der weiblichen Kontrollen ebenfalls vergleichsweise größer (Abbildung 36). In der Analyse wird diesbezüglich ein statistisch signifikanter Unterschied mit $\mathrm{p}=0,06$ jedoch knapp verfehlt.

Der Vergleich zwischen der Gruppe der Kontrollpersonen und der der betreuenden Angehörigen ergibt für die Gesamtstichprobe vergleichbare Mittelwerte und Standardabweichungen. Die männlichen Kontrollpersonen haben durchschnittlich einen geringeren Wert für Depressivität als die betreuenden Söhne, wohingegen die weiblichen Kontrollpersonen mit dem Mittelwert auf der CESD-10 über dem der betreuenden Töchter liegen (Abbildung 37). In der Analyse sind jedoch auf einem Niveau von p $<0,05$ keine statistisch signifikanten Unterschiede der Durchschnittswerte zu belegen. Die Anzahl der über dem cut-off liegenden Personen ist in der Gruppe der Kontrollpersonen und der betreuenden Angehörigen nahezu identisch. 


\subsubsection{Nurses' Observation Scale for Geriatric Patients (NOSGER II)}

In Tabelle 19 werden die NOSGER-Ergebnisse der Kontrollgruppe im Vergleich der mit der Betreuergruppe dargestellt. Dieser Fragebogen, der sich inhaltlich mit dem Grad der Hilfs- bzw. Pflegebedürftigkeit älterer Menschen befasst, wurde von den Studienteilnehmern über ihre Eltern ausgefüllt. So ist es möglich, den mit einer alters- oder krankheitsbedingten Einschränkung des Elternteils einhergehenden Betreuungsbedarf zu objektivieren und einen Vergleich zwischen den gesunden Eltern der Kontrollpersonen und den an der Untersuchung teilnehmenden Parkinsonpatienten anzustellen. Hohe Werte entsprechen dabei einem höheren Grad an elterlicher Hilfsbedürftigkeit.

Tabelle 19: Mittelwerte und Standardabweichungen der NOSGER der Kontrollgruppe im Vergleich mit den Ergebnissen der Betreuergruppe

\begin{tabular}{|l|r|r|r|}
\hline & \multicolumn{1}{|l|}{$\begin{array}{l}\text { Betreuer } \\
\mathrm{n}=50\end{array}$} & $\begin{array}{l}\text { Kontrollen } \\
\mathrm{n}=50\end{array}$ & p-Werte \\
\hline NOSGER gesamt & $\begin{array}{r}54,14 \\
(16,41)\end{array}$ & $\begin{array}{r}39,64 \\
(7,13)\end{array}$ & 0,000000 \\
\hline Gedächtnis & $\begin{array}{r}8,32 \\
(2,73)\end{array}$ & $\begin{array}{r}6,38 \\
(1,56)\end{array}$ & 0,000032 \\
\hline instr. Aktivitäten des tägl. Lebens & 10,26 & 6,92 & \\
& $(4,74)$ & $(2,07)$ & 0,000092 \\
\hline Aktivitäten des täglichen Lebens & 7,16 & 5,28 & \\
& $(2,53)$ & $(0,81)$ & 0,000004 \\
\hline Stimmung & 9,94 & 7,46 & \\
& $(3,46)$ & $(2,24)$ & 0,000079 \\
\hline soziales Verhalten & $\begin{array}{r}10,32 \\
(4,42)\end{array}$ & $\begin{array}{r}6,76 \\
(2,30)\end{array}$ & 0,000010 \\
\hline störendes Verhalten & 8,26 & $\begin{array}{r}6,84 \\
(1,49)\end{array}$ & 0,001028 \\
\hline
\end{tabular}

Sowohl der von den Kontrollpersonen angegebene Gesamtwert als auch die verschiedenen Unterscores der NOSGER liegen unter den entsprechenden Werten der an M. Parkinson erkrankten Eltern der Studienpopulation. In der Analyse lassen sich für den Gesamtwert der Skala sowie für die Teilgebiete „Gedächtnis“, „,instrumentelle Aktivitäten des täglichen Lebens“, „Aktivitäten des täglichen Lebens“, „Stimmung“ und „soziales Verhalten“ auf einem Niveau von $p<0,001$ statistisch signifikante Unterschiede belegen. Das Signifikanzniveau für die Rubrik „störendes Verhalten“ liegt bei $\mathrm{p}<0,05$. In der Abbildung 38 sind die Mittelwerte der NOSGER und ihrer Unterskalen graphisch dargestellt.

\subsubsection{Epworth Sleepiness Scale (ESS)}

Im Folgenden werden die Ergebnisse der Epworth Sleepiness Scale der Kontrollpersonen dargestellt und mit den Werten der betreuenden Angehörigen verglichen. 
Tabelle 20: Vergleich der Ergebnisse der ESS zwischen der Studien- und Kontrollgruppe

\begin{tabular}{|l|r|l|l|l|l|l|}
\hline & \multicolumn{3}{|l|}{ Studiengruppe } & \multicolumn{2}{l|}{ Kontrollpersonen } \\
\cline { 2 - 7 } & $\begin{array}{l}\text { Söhne } \\
\mathrm{n}=19\end{array}$ & $\begin{array}{l}\text { Töchter } \\
\mathrm{n}=31\end{array}$ & $\begin{array}{l}\text { alle } \\
\mathrm{n}=50\end{array}$ & $\begin{array}{l}\text { Söhne } \\
\mathrm{n}=19\end{array}$ & $\begin{array}{l}\text { Töchter } \\
\mathrm{n}=31\end{array}$ & $\begin{array}{l}\text { alle } \\
\mathrm{n}=50\end{array}$ \\
\hline ESS & & & & & & 0 \\
\hline Minimum & 0 & 1 & 0 & 1 & 17 & 17 \\
\hline Maximum & 13 & 18 & 18 & 12 & 6,97 & 6,36 \\
\hline Mittelwert & 5,37 & 7,13 & 6,46 & 5,37 & 3,67 & 3,60 \\
\hline Std.abw. & 3,66 & 3,56 & 3,66 & 3,32 & & \\
\hline
\end{tabular}

Aus der Aufstellung ist ersichtlich, dass die weiblichen Kontrollpersonen bei einer größeren Spannbreite der Einzelwerte einen im Durchschnitt um 1,6 Punkte höheren ESS-Gesamtwert zeigen als die männlichen Probanden. Der Maximalwert liegt bei den Frauen ebenfalls deutlich über dem der Männer. Die Ergebnisse sind in der Abbildung 39 graphisch dargestellt.

Die Mittelwerte der betreuenden Angehörigen und der Kontrollpersonen sind sowohl für die geschlechtsspezifisch getrennten Untergruppen als auch für die Gesamtstichprobe in etwa vergleichbar. Dementsprechend lassen sich durch den Wilcoxon-Mann-Whitney-U-Test auf einem Niveau von $\mathrm{p}<0,05$ auch keine statistisch signifikanten Unterschiede zwischen der Betreuer- und Kontrollgruppe belegen.

\subsubsection{Pittsburgh Sleep Quality Index (PSQI)}

Tabelle 21 stellt die Ergebnisse des Gesamtwertes des Pittsburgh Sleep Quality Index für die Kontrollgruppe im Vergleich mit den entsprechenden Werten der betreuenden Angehörigen dar. Der Grenzwert zur Differenzierung zwischen „guten“ und „schlechten“ Schläfern liegt bei 5 Punkten (gute Schläfer $\leq 5$; schlechte Schläfer $>5$ ).

Tabelle 21: Ergebnisse der PSQI-Gesamtwerte der Kontrollpersonen im Vergleich mit den Ergebnissen der Studiengruppe

\begin{tabular}{|c|c|c|c|c|c|c|}
\hline & \multicolumn{3}{|l|}{ Betreuer } & \multicolumn{3}{|c|}{ Kontrollpersonen } \\
\hline & $\begin{array}{l}\text { Söhne } \\
\mathrm{n}=19\end{array}$ & $\begin{array}{l}\text { Töchter } \\
\mathrm{n}=31\end{array}$ & $\begin{array}{l}\text { alle } \\
\mathrm{n}=50\end{array}$ & $\begin{array}{l}\text { Söhne } \\
\mathrm{n}=19\end{array}$ & $\begin{array}{l}\text { Töchter } \\
\mathrm{n}=30\end{array}$ & $\begin{array}{l}\text { alle } \\
n=49\end{array}$ \\
\hline \multicolumn{7}{|l|}{ PSQI gesamt } \\
\hline Minimum & 1 & 0 & 0 & 1 & 0 & 0 \\
\hline Maximum & 10 & 13 & 13 & 8 & 12 & 12 \\
\hline Mittelwert & 4,42 & 5,23 & 4,92 & 3,79 & 4,43 & 4,18 \\
\hline Std.abw. & 1,95 & 3,11 & 2,73 & 2,04 & 2,75 & 2,50 \\
\hline n über cut-off & 4 & 11 & 15 & 3 & 9 & 12 \\
\hline$\%$ über cut-off & 21,1 & 35,5 & 30,0 & 15,8 & 30,0 & 24,0 \\
\hline
\end{tabular}

Die weiblichen Kontrollpersonen zeigen neben einem höheren durchschnittlichen PSQI-Gesamtwert eine größere Streubreite der Einzelwerte als die männlichen Kontrollen. Diese Unterschiede erreichen jedoch nicht das Signifikanzniveau von $\mathrm{p}<0,05$. 
Im Vergleich mit der Gruppe der betreuenden Angehörigen liegen die Mittelwerte der männlichen und weiblichen Kontrollpersonen sowie der Mittelwert der Gesamtstichprobe unter den entsprechenden Werten der Studiengruppe. Die Analyse verfehlt hierbei allerdings ebenfalls eine statistische Signifikanz.

Insgesamt liegen vergleichsweise weniger Kontrollpersonen als betreuende Angehörige über dem Grenzwert zur Differenzierung zwischen ,guten“ und „schlechten“ Schläfern. Dies gilt nicht nur für die Gesamtstichprobe sondern auch für die geschlechtsspezifischen Untergruppen. Die Abbildungen 40 und 41 veranschaulichen diese Ergebnisse graphisch.

\subsection{Ergebnisse Hypothese 1}

Die erste Hypothese besagt, dass eine hohe filiale Reife zu einem verminderten Belastungserleben des betreuenden Kindes führe. Um diese Hypothese zu belegen, wurde nach Korrelationen sowohl der Filial Anxiety Scale als auch des Filiale-Reife-Bogens mit den das Belastungserleben widerspiegelnden Fragebögen der betreuenden Angehörigen gesucht. Als Messinstrumente hierfür wurden in dieser Untersuchung der Fragebogen zur Belastung durch die Betreuung, der SF-36 (gesundheitsbezogene Lebensqualität) sowie die CESD-10 (Depression) genutzt. Da Unterschiede zwischen den betreuenden Söhnen und Töchtern auftraten, werden die Ergebnisse nicht nur für die Gesamtstichprobe, sondern auch separat für die beiden Geschlechter dargestellt.

\subsubsection{Filial Anxiety Scale und Fragebogen zur Belastung}

Tabelle 22 zeigt die Korrelationen zwischen den Skalenwerten der filialen Angst und dem Belastungserleben der erwachsenen Kinder durch die Betreuung.

Tabelle 22: Korrelation zwischen Filial Anxiety Scale und dem Belastungsbogen

\begin{tabular}{|c|c|c|c|}
\hline \multicolumn{4}{|c|}{$\begin{array}{l}\text { Spearman's Rangkorrelation } \\
{ }^{\circ} \mathrm{p}<0,1 * \mathrm{p}<0,05 * * \mathrm{p}<0,01 * * * \mathrm{p}<0,001\end{array}$} \\
\hline & $\begin{array}{l}\text { Söhne } \\
\text { Belastung }\end{array}$ & $\begin{array}{l}\text { Töchter } \\
\text { Belastung }\end{array}$ & $\begin{array}{l}\text { alle } \\
\text { Belastung }\end{array}$ \\
\hline Filial Anxiety Scale A & 0,11 & 0,33 & ${ }^{\circ} 0,28$ \\
\hline Filial Anxiety Scale B & 0,33 & $* 0,39$ & $* * 0,38$ \\
\hline
\end{tabular}

Während bei den Söhnen weder die FAA noch die FAB mit dem Fragebogen zur Belastung durch die Betreuung korrelieren, besteht in der Gruppe der betreuenden Töchter ein mäßiger, aber statistisch signifikanter positiver Zusammenhang zwischen der Filial Anxiety Scale B und dem Belastungserleben $(r=0,33 ; \mathrm{p}=0,035)$. Für die Gesamtstichprobe wird eine statistisch signifikante Korrelation der Filial Anxiety Scale A mit dem Belastungsbogen nur knapp verpasst $(r=0,28$; 
$\mathrm{p}=0,051$ ). Eine eindeutige positive Korrelation besteht hingegen mit $\mathrm{p}=0,007$ zwischen der $\mathrm{FAB}$ und dem Belastungsbogen (Abb. 42).

\subsubsection{Filial Anxiety Scale und SF-36}

Im Folgenden werden die Korrelationen zwischen der Filial Anxiety Scale und dem Fragebogen zur gesundheitsbezogenen Lebensqualität (SF-36) dargestellt.

Tabelle 23: Korrelation zwischen der Filial Anxiety Scale und dem SF-36

\begin{tabular}{|c|c|c|c|c|c|c|c|c|}
\hline \multicolumn{9}{|c|}{$\begin{array}{l}\text { Spearman's Rangkorrelation } \\
* \mathrm{p}<0,05 \quad * * \mathrm{p}<0,01 \quad * * * \mathrm{p}<0,001\end{array}$} \\
\hline & KöFu & KöRo & KöSch & AlGes & Vital & SoFu & EmRo & PsyWo \\
\hline \multicolumn{9}{|l|}{ Söhne } \\
\hline FAA & 0,35 & 0,07 & 0,14 & 0,04 & 0,31 & 0,12 & 0,07 & 0,03 \\
\hline FAB & 0,11 & $-0,007$ & 0,11 & 0,11 & 0,06 & 0,37 & 0,45 & 0,07 \\
\hline \multicolumn{9}{|l|}{ Töchter } \\
\hline FAA & $-0,24$ & $-0,08$ & $-0,05$ & 0,02 & $-0,23$ & $-0,12$ & $*_{-} 0,39$ & $-0,25$ \\
\hline FAB & $* *_{-} 0,53$ & $-0,31$ & $-0,26$ & $-0,10$ & 0,06 & 0,13 & 0,003 & $-0,02$ \\
\hline \multicolumn{9}{|l|}{ alle } \\
\hline FAA & $-0,01$ & $-0,03$ & $-0,003$ & 0,03 & $-0,05$ & $-0,05$ & $-0,22$ & $-0,17$ \\
\hline FAB & $*_{-} 0,30$ & $-0,25$ & $-0,13$ & $-0,009$ & 0,02 & 0,20 & 0,15 & $-0,003$ \\
\hline
\end{tabular}

Während in der Gesamtstichprobe kein statistisch signifikanter Zusammenhang zwischen der FAA und den Rubriken des SF-36 besteht, korrelieren die FAB und die körperliche Funktionsfähigkeit mit $\mathrm{p}=0,035$ negativ miteinander. In der Gruppe der betreuenden Söhne bestehen weder für die FAA noch die FAB signifikante Korrelationen mit den SF-36-Unterskalen. Bei den Töchtern hingegen lässt sich je eine negative Korrelation zwischen der FAA und der emotionalen Rollenfunktion ( $r=-0,39$; $\mathrm{p}=0,028)$ sowie zwischen der $\mathrm{FAB}$ und der körperlichen Funktionsfähigkeit $(\mathrm{r}=-0,53 ; \mathrm{p}=0,002)$ belegen.

\subsubsection{Filial Anxiety Scale und CESD-10}

In Tabelle 24 sind die Rangkorrelationen nach Spearman von der Filial Anxiety Scale A und B mit der CESD-10 dargestellt.

Tabelle 24: Korrelation zwischen Filial Anxiety Scale und der CESD-10

\begin{tabular}{|c|c|c|c|}
\hline \multicolumn{4}{|c|}{$\begin{array}{l}\text { Spearman's Rangkorrelation } \\
{ }_{0} \mathrm{p}<0,1 * \mathrm{p}<0,05 * *_{\mathrm{p}}<0,01 \quad * * * \mathrm{p}<0,001\end{array}$} \\
\hline & $\begin{array}{l}\text { Söhne } \\
\text { CESD-10 }\end{array}$ & $\begin{array}{l}\text { Töchter } \\
\text { CESD-10 }\end{array}$ & $\begin{array}{l}\text { alle } \\
\text { CESD-10 }\end{array}$ \\
\hline Filial Anxiety Scale A & $-0,04$ & $* 0,45$ & ${ }^{\circ} 0,28$ \\
\hline Filial Anxiety Scale B & $-0,04$ & $* 0,40$ & 0,24 \\
\hline
\end{tabular}


Auf dem angestrebten Signifikanzniveau von $\mathrm{p}<0,05$ bestehen bei den betreuenden Töchtern sowohl zwischen der Skala A als auch der Skala B der Filial Anxiety Scale und der CESD-10 statistisch signifikante positive Korrelationen (FAA: $p=0,011$; FAB: $p=0,027$ ). In der Gruppe der Söhne lässt sich diesbezüglich kein Zusammenhang feststellen. Für die Gesamtstichprobe wird das angestrebte Signifikanzniveau bezüglich der Korrelation zwischen FAA und CESD-10 mit p=0,052 lediglich knapp verfehlt.

\subsubsection{Filiale-Reife-Bogen und Fragebogen zur Belastung}

Im Folgenden werden die Rangkorrelationen zwischen den Faktoren der filialen Reife (LFMS-A) und dem Belastungserleben der betreuenden Kinder dargestellt.

Tabelle 25: Korrelation zwischen der LFMS-A und dem Belastungsbogen

\begin{tabular}{|c|c|c|c|}
\hline \multicolumn{4}{|c|}{$\begin{array}{l}\text { Spearman's Rangkorrelation } \\
{ }^{\circ} \mathrm{p}<0,1 * \mathrm{p}<0,05 * * \mathrm{p}<0,01 * * * \mathrm{p}<0,001\end{array}$} \\
\hline & $\begin{array}{l}\text { Söhne } \\
\text { Belastung }\end{array}$ & $\begin{array}{l}\text { Töchter } \\
\text { Belastung }\end{array}$ & $\begin{array}{l}\text { alle } \\
\text { Belastung }\end{array}$ \\
\hline filiale Liebe & 0,10 & 0,002 & 0,04 \\
\hline fil. Verpflichtungsgefühl & ${ }^{\circ} 0,44$ & $* * 0,46$ & $* * 0,44$ \\
\hline filiale Hilfsbereitschaft & $-0,01$ & $-0,10$ & 0,03 \\
\hline filiale Hilfe & 0,15 & 0,09 & 0,16 \\
\hline filiale Autonomie & $-0,16$ & $-0,25$ & $-0,21$ \\
\hline elterliche Besorgnis & $-0,39$ & $-0,16$ & $-0,26$ \\
\hline familiäre Solidarität & 0,28 & $*_{-0,42}$ & $-0,13$ \\
\hline
\end{tabular}

Während die Analyse für das filiale Verpflichtungsgefühl der Töchter und der betreuenden Angehörigen insgesamt auf einem Niveau von $\mathrm{p}<0,01$ eindeutig statistisch signifikante positive Korrelationen mit der empfundenen Belastung belegt (Abb. 43), verfehlt die entsprechende Korrelation in der Gruppe der betreuenden Söhne knapp das angestrebte Signifikanzniveau von $\mathrm{p}<0,05(\mathrm{r}=0,44 ; \mathrm{p}=0,057)$. Bei den Töchtern korreliert zudem die Einschätzung der familiären Solidarität negativ mit dem Belastungserleben durch die Betreuung $(r=-0,42 ; p=0,02)$.

\subsubsection{Filiale-Reife-Bogen und SF-36}

In der Tabelle 26 sind die Korrelationen der Ausprägung der Filialen Reife (LFMS-A) mit der Gesundheitsbezogenen Lebensqualität (SF-36) der betreuenden Angehörigen insgesamt dargestellt. Zusätzlich werden in den Abbildungen 44 und 45 für die beiden Geschlechter getrennt diejenigen statistischen Zusammenhänge gezeigt, die das angestrebte Signifikanzniveau von $p<0,05$ erreichen oder dieses nur knapp verfehlen. 
Tabelle 26: Korrelation zwischen der LFMS-A und dem SF-36 für die Gesamtstichprobe

\begin{tabular}{|l|l|r|r|r|r|r|r|r|}
\hline $\begin{array}{l}\text { Spearman's Rangkorrelation } \\
* \mathrm{p}<0,05\end{array} *{ }^{*} \mathrm{p}<0,01$ & $* * * \mathrm{p}<0,001$ \\
\hline & \multicolumn{1}{|c|}{ KöFu } & \multicolumn{1}{l|}{ KöRo } & \multicolumn{1}{|c|}{ KöSch } & \multicolumn{1}{c|}{ AlGes } & \multicolumn{1}{c|}{ Vital } & \multicolumn{1}{c|}{ SoFu } & EmRo & PsyWo \\
\hline FL & $-0,23$ & $-0,16$ & 0,02 & $-0,06$ & 0,007 & 0,08 & 0,15 & $* 0,29$ \\
\hline FO & $*-0,31$ & $-0,22$ & 0,02 & $-0,06$ & $-0,06$ & 0,09 & 0,02 & 0,10 \\
\hline FHN & $-0,23$ & $-0,23$ & $-0,07$ & $-0,04$ & 0,0002 & 0,11 & 0,26 & $* 0,29$ \\
\hline FH & $*-0,33$ & $-0,25$ & $-0,07$ & 0,05 & $-0,07$ & 0,13 & 0,21 & 0,18 \\
\hline FA & $* 0,36$ & $* 0,32$ & 0,15 & $* * 0,39$ & 0,14 & 0,14 & 0,07 & $-0,05$ \\
\hline PC & 0,06 & 0,004 & 0,02 & 0,16 & 0,23 & 0,26 & 0,13 & $* * 0,44$ \\
\hline FS & 0,05 & 0,08 & 0,18 & 0,11 & 0,23 & $* 0,31$ & $* 0,29$ & 0,28 \\
\hline
\end{tabular}

Auf dem angestrebten Signifikanzniveau von $p<0,05$ korrelieren in der Betreuergruppe die Faktoren „filiale Liebe“ und „filiale Hilfsbereitschaft“ jeweils mäßig stark positiv mit dem „psychischen Wohlbefinden" (FL: r=0,29; $p=0,04 ; F H N$ : $r=0,29 ; p=0,04$ ). Negative Korrelationen lassen sich für die Reife-Faktoren „filiales Verpflichtungsgefühl“ und „filiale Hilfe“ im Zusammenhang mit der „körperlichen Funktionsfähigkeit“ belegen (FO: r=-0,31; p=0,03; FH: r=-0,33; p=0,02). Die „filiale Autonomie" der betreuenden Angehörigen steht in einem statistisch signifikanten positiven Zusammenhang mit der „körperlichen Funktionsfähigkeit“ ( $r=0,36 ; p=0,011)$, der „,körperlichen Rollenfunktion“ ( $r=0,32 ; \mathrm{p}=0,026)$ sowie der ,allgemeinen Gesundheit“ ( $r=0,39 ; \mathrm{p}=0,005)$. Die empfundene „elterliche Besorgnis“ korreliert mit $\mathrm{p}=0,0011$ eindeutig positiv mit dem „psychischen Wohlergehen“ der Betreuer. Darüber hinaus sind zwischen der „familiären Solidarität“ und der „Sozialen Funktionsfähigkeit“ sowie der „emotionalen Rollenfunktion“ statistisch signifikante positive Korrelationen festzustellen (SoFu: $r=0,31 ; p=0,03$; EmRo: $r=0,29 ; p=0,04$ ).

In der Gruppe der betreuenden Söhne erreicht lediglich die Korrelation zwischen der „filialen Autonomie“ und der ,allgemeinen Gesundheit“ der Söhne mit p=0,02 statistische Signifikanz.

Die Korrelationen der betreuenden Töchter stimmen weitestgehend mit denen der Gesamtstichprobe überein. Folgende Rubriken korrelieren hier statistisch signifikant miteinander: „filiale Liebe“ und „psychisches Wohlbefinden“ ( $r=0,38 ; \mathrm{p}=0,04)$ sowie „filiales Verpflichtungsgefühl“ und „körperliche Funktionsfähigkeit“ ( $\mathrm{r}=0,44 ; \mathrm{p}=0,013)$ mäßig stark positiv, „filiale Hilfe“ und „körperliche Funktionsfähigkeit“ deutlich negativ ( $\mathrm{r}=-0,46 ; \mathrm{p}=0,01)$ und ,elterliche Besorgnis“ und ,psychisches Wohlbefinden" deutlich positiv miteinander $(r=0,53 ; p=0,002)$. Die von den Töchtern empfundene „familiäre Solidarität“ korreliert positiv mit ihrer „Vitalität“ ( $\mathrm{r}=0,44 ; \mathrm{p}=0,012)$, „sozialen Funktionsfähigkeit“ ( $r=0,45 ; p=0,011)$ und „emotionalen Rollenfunktion“ ( $r=0,43 ; p=0,016)$. Darüber hinaus verfehlen in der Gruppe der betreuenden Töchter die Zusammenhänge zwischen der „filialen Liebe“ und der „emotionalen Rollenfunktion“ ( $\mathrm{r}=0,34 ; \mathrm{p}=0,06)$, dem ,filialen Verpflichtungsgefühl“ und dem „psychischen Wohlbefinden“ $(r=0,34 ; \mathrm{p}=0,06)$ sowie der Zusammenhang zwischen der empfundenen „familiären Solidarität“ und dem „psychischen Wohlergehen“ ( $r=0,34 ; p=0,06)$ nur knapp das angestrebte Signifikanzniveau von $\mathrm{p}<0,05$. 


\subsubsection{Filiale-Reife-Bogen und CESD-10}

Tabelle 27 bildet die Zusammenhänge zwischen den Faktoren der filialen Reife (LFMS-A) und einer möglichen Depressivität (CESD-10) der betreuenden Angehörigen ab.

Tabelle 27: Korrelation zwischen der LFMS-A und der CESD-10

\begin{tabular}{|c|c|c|c|}
\hline \multicolumn{4}{|c|}{$\begin{array}{l}\text { Spearman's Rangkorrelation } \\
{ }^{\circ}<<0,1 * p<0,05 \quad * * p<0,01 \quad * * * p<0,001\end{array}$} \\
\hline & $\begin{array}{l}\text { Söhne } \\
\text { CESD-10 }\end{array}$ & $\begin{array}{l}\text { Töchter } \\
\text { CESD-10 }\end{array}$ & $\begin{array}{l}\text { alle } \\
\text { CESD-10 }\end{array}$ \\
\hline filiale Liebe & 0,12 & $-0,07$ & 0,007 \\
\hline fil. Verpflichtungsgefühl & $-0,05$ & 0,22 & 0,10 \\
\hline filiale Hilfsbereitschaft & 0,01 & $-0,18$ & $-0,07$ \\
\hline filiale Hilfe & $-0,07$ & 0,09 & 0,05 \\
\hline filiale Autonomie & $-0,37$ & $-0,02$ & $-0,14$ \\
\hline elterliche Besorgnis & $-0,16$ & ${ }^{\circ}-0,35$ & ${ }^{\circ}-0,26$ \\
\hline familiäre Solidarität & $-0,14$ & ${ }^{\circ}-0,35$ & $*-0,29$ \\
\hline
\end{tabular}

Auf dem angestrebten Signifikanzniveau von $\mathrm{p}<0,05$ korreliert lediglich die familiäre Solidarität negativ mit der Depressivität der betreuenden Söhne und Töchter ( $r=-0,29 ; \mathrm{p}=0,04)$. Die negativen Korrelationen zwischen der von den Töchtern empfundenen elterlichen Besorgnis und der Depressionsskala sowie zwischen der familiären Solidarität und der Depressionsskala verfehlen mit $\mathrm{p}=0,053$ bzw. $\mathrm{p}=0,055$ nur knapp die statistische Signifikanz. In der Gruppe der betreuenden Söhne schließt die Analyse einen statistisch signifikanten Zusammenhang zwischen den Faktoren der filialen Reife und einer möglichen Depressivität des Betreuers aus.

\subsubsection{Zusammenfassung Ergebnisse Hypothese 1}

Zusammenfassend kann festgestellt werden, dass die Filial Anxiety Scale und die Louvain Filial Maturity Scale A in unterschiedlichem Ausmaß mit den drei das Belastungserleben widerspiegelnden Fragebögen korrelieren.

Während die FAA weniger häufig als die FAB mit dem allgemeinen Belastungserleben der betreuenden Angehörigen korreliert, betreffen die diesbezüglichen statistischen Zusammenhänge bei den sieben Faktoren des Filiale-Reife-Bogens gehäuft das filiale Verpflichtungsgefühl, die filiale Autonomie und die familiäre Solidarität. Die Rubriken „körperliche Funktionsfähigkeit“ und „psychisches Wohlergehen“ korrelieren im Vergleich mit den übrigen Rubriken des SF-36 am häufigsten mit den Bögen zur filialen Angst bzw. Reife. Die CESD-10 korreliert höher mit der Filial Anxiety Scale als mit dem Filiale-Reife-Bogen.

Generell hat die Ausprägung der filialen Reife bei den betreuenden Töchtern einen deutlich stärkeren Einfluss auf Belastung, Lebensqualität und Depressivität als bei den Söhnen. 


\subsection{Ergebnisse Hypothese 2}

Die zweite Hypothese lautet, dass die Filial Anxiety Scale besser mit dem Belastungserleben des betreuenden Kindes korreliere als der Filiale-Reife-Bogen. Da es nicht möglich ist, zwei Korrelationen durch ein statistisches Verfahren miteinander zu korrelieren, konnte zur Überprüfung dieser Hypothese kein statistisches Testverfahren angewandt werden. Die Häufigkeit und Ausprägung der Korrelationen zwischen der Filial Anxiety Scale und dem Fragebogen zur Belastung durch die Betreuung wurden daher im Folgenden lediglich deskriptiv mit den Korrelationen zwischen dem Filiale-Reife-Bogen und dem Belastungsbogen verglichen.

In Tabelle 28 sind diesbezüglich diejenigen Rangkorrelationen der beiden Skalen dargestellt, die das angestrebte Signifikanzniveau von $p<0,05$ erreichen oder lediglich knapp verfehlen.

Tabelle 28: Vergleich der Korrelationen der Filial Anxiety Scale mit dem Belastungsbogen und der LFMS-A mit dem Belastungsbogen

\begin{tabular}{|c|c|c|c|c|c|c|}
\hline \multicolumn{7}{|c|}{$\begin{array}{l}\text { Spearman's Rangkorrelation } \\
{ }^{\circ} \mathrm{p}<0,1 * \mathrm{p}<0,05 \quad * * \mathrm{p}<0,01 \quad * * * \mathrm{p}<0,001 \text { n.s. }=\text { nicht signifikant }\end{array}$} \\
\hline & \multicolumn{2}{|l|}{ Söhne } & \multicolumn{2}{|c|}{ Töchter } & \multicolumn{2}{|l|}{ alle } \\
\hline & $\mathbf{r}$ & $\mathbf{p}$ & $\mathbf{r}$ & $\mathbf{p}$ & $\mathbf{r}$ & $\mathbf{p}$ \\
\hline \multicolumn{7}{|l|}{ Filial Anxiety Scale } \\
\hline FAA \& Belastungsbogen & n.s. & n.s. & n.s. & n.s. & 0,28 & ${ }^{\circ} 0,0505$ \\
\hline FAB \& Belastungsbogen & n.s. & n.s. & 0,39 & $* 0,035$ & 0,38 & $* * 0,007$ \\
\hline \multicolumn{7}{|l|}{ Filiale-Reife-Bogen } \\
\hline FO \& Belastungsbogen & 0,44 & ${ }^{\circ} 0,057$ & 0,46 & $* * 0,010$ & 0,44 & $* * 0,002$ \\
\hline FS \& Belastungsbogen & n.s. & n.s. & $-0,42$ & $* 0,021$ & n.s. & n.s. \\
\hline
\end{tabular}

Bezogen auf die Gesamtstichprobe verpasst die Skala A der Filial Anxiety Scale mit $p=0,051$ nur knapp die angestrebte statistische Signifikanz mit dem Belastungsbogen, während die FAB deutlich positiv mit dem Belastungserleben der betreuenden Angehörigen korreliert ( $\mathrm{p}<0,01)$. Vergleichbar ist die Verteilung der Korrelationen in der Untergruppe der betreuenden Töchter: Die FAA korreliert nicht, die FAB positiv mit dem Belastungsbogen. Bei den Söhnen lassen sich keine statistisch signifikanten Zusammenhänge zwischen der Ausprägung der filialen Angst und der durch die Betreuung erlebten Belastung feststellen.

Von den sieben Unterskalen des Filiale-Reife-Bogens korreliert bei den betreuenden Angehörigen insgesamt lediglich der Faktor „filiales Verpflichtungsgefühl“ mit dem Belastungsbogen, bei den Töchtern die Faktoren „filiales Verpflichtungsgefühl“ und „familiäre Solidarität“. Diese Zusammenhänge sind signifikant (FO: $p<0,01$; FS: $p=0,021$ ). Die Korrelation zwischen dem „filialen Verpflichtungsgefühl“ der Söhne und ihrem Belastungserleben verpasst mit $\mathrm{p}=0,057$ wiederum knapp das angestrebte Signifikanzniveau. 


\subsubsection{Zusammenfassung Ergebnisse Hypothese 2}

Anhand der dargestellten Untersuchungsergebnisse kann abschließend von einem besseren Zusammenhang zwischen der Filial Anxiety Scale und dem Fragebogen zur Belastung durch die Betreuung ausgegangen werden als vergleichend mit dem Filiale-Reife-Bogen A.

\subsection{Ergebnisse Hypothese 3}

Die dritte Hypothese besagt, dass Schlafstörungen sowohl bei den betreuenden Kindern als auch bei den Patienten zu einer Verstärkung des Belastungserlebens und einer Einschränkung der gesundheitsbezogenen Lebensqualität führe. Um diese Hypothese zu überprüfen, wurden Korrelationen zwischen den „Schlafbögen“ und dem Fragebogen zur Belastung durch die Betreuung sowie dem Fragebogen zur Gesundheitsbezogenen Lebensqualität (SF-36) errechnet. Als „Schlafbögen“ zählen in der Gruppe der betreuenden Angehörigen die Epworth Sleepiness Scale (ESS) und der Pittsburgh Sleep Quality Index (PSQI), in der Patientengruppe zusätzlich noch die Parkinson's Disease Sleep Scale (PDSS).

Da teilweise große geschlechtsspezifische Unterschiede in Anzahl und Ausprägung der Korrelationen auftraten, werden die Ergebnisse wieder nicht nur jeweils für die Gesamtstichprobe sondern auch separat für Söhne und Töchter bzw. männliche und weibliche Parkinsonpatienten dargestellt.

\subsubsection{ESS/PSQI und Belastungsbogen (Betreuer)}

Im Folgenden werden für die Gruppe der betreuenden Angehörigen die Korrelationen zwischen der Epworth Sleepiness Scale (ESS) und dem Fragebogen zur Belastung durch die Betreuung sowie die Zusammenhänge zwischen dem Pittsburgh Sleep Quality Index (PSQI) und dem Belastungsbogen dargestellt.

Tabelle 29: Korrelationen der ESS und der PSQI der Betreuer mit dem Belastungsbogen

\begin{tabular}{|l|l|r|r|}
\hline $\begin{array}{l}\text { Spearman's Rangkorrelation } \\
* \mathrm{p}<0,05 * * \mathrm{p}<0,01 * * * \mathrm{p}<0,001\end{array}$ \\
\hline & $\begin{array}{l}\text { Söhne } \\
\text { Belastung }\end{array}$ & $\begin{array}{l}\text { Töchter } \\
\text { Belastung }\end{array}$ & $\begin{array}{l}\text { alle } \\
\text { Belastung }\end{array}$ \\
\hline ESS & 0,23 & $-0,17$ & 0,04 \\
\hline PSQI gesamt & $-0,10$ & 0,30 & 0,17 \\
\hline subj. Schlafqualität & 0,21 & 0,32 & $* 0,29$ \\
\hline Schlaflatenz & $-0,03$ & 0,27 & 0,14 \\
\hline Schlafdauer & $-0,32$ & 0,15 & $-0,08$ \\
\hline Schlafeffizienz & $-0,14$ & 0,28 & 0,11 \\
\hline Schlafstörungen & 0,31 & 0,08 & 0,16 \\
\hline Schlafmittelkonsum & $\mathrm{x}$ & 0,15 & 0,14 \\
\hline Tagesschläfrigkeit & 0,22 & 0,20 & 0,20 \\
\hline
\end{tabular}


Auf dem angestrebten Signifikanzniveau von $\mathrm{p}<0,05$ können weder für die ESS noch für den PSQIGesamtwert statistisch signifikante Zusammenhänge mit dem Belastungsbogen gezeigt werden. Lediglich die subjektive Schlafqualität der Gesamtstichprobe (Unterskala 1 des PSQI) korreliert positiv mit dem Belastungserleben der betreuenden Söhne und Töchter $(r=0,29 ; p=0,04)$.

\subsubsection{ESS/PSQI und SF-36 (Betreuer)}

Tabelle 30 stellt die Korrelationen zwischen der Epworth Sleepiness Scale (ESS) und dem SF-36 sowie die Korrelationen des PSQI-Gesamtwertes mit dem SF-36 dar. Die Spearmans' Rangkorrelationen der Unterskalen des PSQI mit dem SF-36 sind im Anhang in den Abbildungen 4648 zu finden.

Tabelle 30: Korrelation zwischen dem ESS/PSQI-Gesamtwert und dem SF-36 (Betreuer)

\begin{tabular}{|c|c|c|c|c|c|c|c|c|}
\hline \multicolumn{9}{|c|}{$\begin{array}{l}\text { Spearman's Rangkorrelation } \\
* \mathrm{p}<0,05 \quad * * \mathrm{p}<0,01 \quad * * * \mathrm{p}<0,001\end{array}$} \\
\hline & KöFu & KöRo & KöSch & AlGes & Vital & SoFu & EmRo & PsyWo \\
\hline \multicolumn{9}{|l|}{ ESS } \\
\hline alle & $-0,20$ & $-0,11$ & $-0,14$ & 0,04 & 0,08 & 0,13 & 0,03 & 0,24 \\
\hline Söhne & $-0,20$ & $* 0,53$ & 0,05 & $-0,01$ & 0,33 & 0,21 & 0,07 & 0,44 \\
\hline Töchter & $-0,16$ & $-0,22$ & $-0,19$ & 0,08 & 0,02 & 0,09 & 0,02 & 0,15 \\
\hline \multicolumn{9}{|l|}{ PSQI } \\
\hline alle & $* * *-0,49$ & $* *-0,43$ & $-0,23$ & $* *-0,37$ & $* *-0,42$ & $-0,17$ & $-0,22$ & $*_{-} 0,29$ \\
\hline Söhne & $-0,08$ & $-0,04$ & -016 & $*_{-} 0,52$ & $-0,22$ & $-0,05$ & $-0,17$ & 0,03 \\
\hline Töchter & $* * *-0,68$ & $* * *-0,57$ & $-0,31$ & $-0,31$ & $* *-0,48$ & $-0,20$ & $-0,25$ & $*_{-0,42}$ \\
\hline
\end{tabular}

Die Epworth Sleepiness Scale steht lediglich mit der „körperlichen Rollenfunktion“ der betreuenden Söhne in einem statistisch signifikanten Zusammenhang ( $r=0,53$; $p=0,02)$.

Der PSQI-Gesamtwert korreliert hingegen deutlich signifikant mit folgenden Rubriken des SF-36: in der Gesamtstichprobe stark negativ mit der „körperlichen Funktionsfähigkeit“ ( $r=-0,49 ; p=0,0004)$, mittel hoch negativ mit der „körperlichen Rollenfunktion“ ( $r=-0,43 ; \mathrm{p}=0,002)$, der ,allgemeinen Gesundheit" ( $r=-0,37 ; p=0,009)$ und „Vitalität“ $(r=-0,42 ; p=0,002)$, sowie mäßig hoch negativ mit dem „psychischen Wohlbefinden“ ( $r=-0,29 ; \mathrm{p}=0,04)$. Bei den betreuenden Töchtern erweisen sich die stark negativen Korrelationen mit der „körperlichen Funktionsfähigkeit“ ( $\mathrm{r}=-0,68 ; \mathrm{p}=0,00003)$ und „körperlichen Rollenfunktion“ ( $\mathrm{r}=-0,57 ; \mathrm{p}=0,0009)$ auf einem Niveau von $\mathrm{p}<0,001$ als deutlich statistisch signifikant. Die „Vitalität“ korreliert mittel hoch negativ $(\mathrm{r}=-0,48 ; \mathrm{p}=0,006)$ und das „psychische Wohlbefinden“ mäßig hoch negativ mit der allgemeinen Schlafqualität ( $r=-0,42 ; p=0,02)$. Bei den betreuenden Söhnen erreicht lediglich der Zusammenhang mit der ,allgemeinen Gesundheit“ $(r=-0,52 ; p=0,02)$ das angestrebte Signifikanzniveau von $\mathrm{p}<0,05$. Einige dieser Ergebnisse sind im Anhang in den Abbildungen 49-52 graphisch dargestellt. 


\subsubsection{ESS/PSQI/PDSS und SF-36 (Patienten)}

Im Folgenden werden die Zusammenhänge zwischen dem Schlafverhalten der Patienten und ihrer Gesundheitsbezogenen Lebensqualität (SF-36) dargestellt.

Tabelle 31: Korrelation zwischen der ESS und dem SF-36, dem PSQI-Gesamtwert und dem SF-36 sowie dem PDSS-Gesamtwert und dem SF-36 (Patienten)

\begin{tabular}{|c|c|c|c|c|c|c|c|c|}
\hline \multicolumn{9}{|c|}{$\begin{array}{l}\text { Spearman's Rangkorrelation } \\
{ }^{\circ}<<0,1 * p<0,05 * * p<0,01 \quad * * * p<0,001\end{array}$} \\
\hline & KöFu & KöRo & KöSch & AlGes & Vital & SoFu & EmRo & PsyWo \\
\hline \multicolumn{9}{|l|}{ ESS } \\
\hline alle & $* * *_{-} 0,52$ & $-0,27$ & $-0,22$ & ${ }^{\circ}-0,27$ & $-0,11$ & $-0,12$ & $-0,13$ & $-0,10$ \\
\hline männl. & $* * *-0,60$ & $* *-0,51$ & $-0,14$ & $*_{-} 0,41$ & $-0,27$ & $-0,24$ & $*_{-0,39}$ & $-0,34$ \\
\hline weibl. & $*_{-0,50}$ & $-0,04$ & $-0,33$ & $-0,08$ & 0,03 & $-0,06$ & 0,12 & 0,04 \\
\hline \multicolumn{9}{|l|}{ PSQI } \\
\hline alle & $-0,20$ & $-0,04$ & $*_{-} 0,30$ & $*_{-0,29}$ & $-0,19$ & $-0,24$ & 0,01 & $-0,19$ \\
\hline männl. & $-0,16$ & $-0,07$ & $-0,31$ & $-0,32$ & $-0,23$ & $-0,18$ & 0,03 & $-0,25$ \\
\hline weibl. & $-0,24$ & 0,04 & $-0,20$ & $-0,36$ & $-0,15$ & $-0,23$ & 0,09 & 0,00 \\
\hline \multicolumn{9}{|l|}{ PDSS } \\
\hline alle & $* 0,34$ & 0,28 & $* * * 0,47$ & $* 0,31$ & $* * 0,44$ & $* 0,36$ & 0,24 & $* * 0,39$ \\
\hline männl. & ${ }^{\circ} 0,36$ & 0,31 & 0,36 & 0,27 & $* * 0,54$ & 0,36 & 0,30 & $* * 0,50$ \\
\hline weibl. & 0,27 & 0,23 & $* * 0,56$ & 0,38 & 0,39 & 0,38 & 0,16 & 0,39 \\
\hline
\end{tabular}

Auf einem Signifikanzniveau von $\mathrm{p}<0,001$ korreliert die Epworth Sleepiness Scale stark negativ mit der „,körperlichen Funktionsfähigkeit“ des gesamten Patientenkollektivs $(r=-0,52 ; p=0,0002)$ sowie der männlichen Patienten ( $r=-0,60 ; \mathrm{p}=0,0007)$. Der negative Zusammenhang zwischen der ESS und der „körperlichen Rollenfunktion“ ( $r=-0,51 ; p=0,006)$ der männlichen Patienten ist mit $p<0,01$ ebenfalls deutlich statistisch signifikant, der Zusammenhang zwischen ESS und der „allgemeinen Gesundheit“ $(\mathrm{r}=-0,41 ; \mathrm{p}=0,03)$ sowie der ,emotionalen Rollenfunktion“ ( $\mathrm{r}=-0,39 ; \mathrm{p}=0,04)$ mäßig stark signifikant (Abbildungen 53-55). In der Gruppe der weiblichen Patienten erreicht lediglich die Korrelation mit der „körperlichen Funktionsfähigkeit“ ( $r=-0,50 ; p=0,03)$ das angestrebte Signifikanzniveau von $p<0,05$. Darüber hinaus erreicht die negative Korrelation der ESS mit der ,,allgemeinen Gesundheit“ ( $r=-0,27$; $\mathrm{p}=0,06)$ des gesamten Patientenkollektivs das statistisch signifikante Niveau von $\mathrm{p}<0,05$ nur knapp nicht.

Der Gesamtwert des Pittsburgh Sleep Quality Index’ korreliert lediglich mit den „körperlichen Schmerzen“ ( $(r=-0,30 ; p=0,03)$ und der ,allgemeinen Gesundheit“ $(r=-0,29 ; p=0,04)$ der Patienten auf einem Niveau von $\mathrm{p}<0,05$ statistisch signifikant (Abbildung 56).

Die Parkinson's Disease Sleep Scale steht mit den „körperlichen Schmerzen“ $(r=0,47 ; p=0,0007)$ der Patienten in stark positivem, mit der „Vitalität“ $(\mathrm{r}=0,44 ; \mathrm{p}=0,002)$ und dem „psychischen Wohlbefinden“ $(\mathrm{r}=0,39 ; \mathrm{p}=0,005)$ in mittel hoch positiven sowie mit der „körperlichen Funktionsfähigkeit“ $(\mathrm{r}=0,34 ; \mathrm{p}=0,02)$, der ,allgemeinen Gesundheit“ $(\mathrm{r}=0,31 ; \mathrm{p}=0,03)$ und der „sozialen Funktionsfähigkeit“ ( $r=0,36 ; p=0,012)$ der Patienten in mäßig hoch positivem statistisch signifikantem Zusammenhang (Abbildungen 57-60). In der Untergruppe der männlichen Patienten 
lassen sich auf einem Niveau von $\mathrm{p}<0,01$ eindeutige signifikante positive Korrelationen mit der „Vitalität“ $(r=0,54 ; p=0,003)$ und dem ,psychischen Wohlbefinden“ $(r=0,50 ; p=0,008)$ belegen. Die „körperlichen Schmerzen“ der Patientinnen stehen mit $\mathrm{p}=0,007$ auf gleichem Niveau in statistisch signifikantem Zusammenhang mit der PDSS ( $r=0,56 ; p=0,007)$. Darüber hinaus verpassen die Korrelationen der PDSS mit den folgenden Rubriken des SF-36 nur knapp das angestrebte Signifikanzniveau von $\mathrm{p}<0,05$ : „körperliche Rollenfunktion“ ( $r=0,28 ; p=0,054)$ des Gesamtkollektivs und ,körperliche Funktionsfähigkeit“ $(r=0,36 ; \mathrm{p}=0,06)$ der männlichen Patienten.

\subsubsection{Zusammenfassung Ergebnisse Hypothese 3}

Zusammenfassend lässt sich feststellen, dass der Fragebogen zur Belastung durch die Betreuung eher schlecht mit der allgemeinen Schlafqualität der betreuenden Kinder korreliert. Eindeutige Zusammenhänge mit dem Gesamtwert des Pittsburgh Sleep Quality Index' lassen sich hingegen für die Rubriken der Gesundheitsbezogenen Lebensqualität belegen. Hierbei liegen sowohl die Anzahl als auch die Stärke der Korrelationen bei den Töchtern bedeutend höher als in der Gruppe der Söhne. Die durch die Epworth Sleepiness Scale abgebildete Tagesschläfrigkeit scheint keinen großen Einfluss auf das Belastungserleben und die Lebensqualität der betreuenden Angehörigen zu haben.

Für die Gruppe der an der Untersuchung teilnehmenden Patienten bleibt zusammenfassend festzustellen, dass bei den männlichen Patienten häufiger und stärkere statistisch signifikante Korrelationen der ESS mit den Dimensionen der Gesundheitsbezogenen Lebensqualität auftreten als bei den Patientinnen. Der Pittsburgh Sleep Quality Index scheint allgemein eher schlecht mit dem SF36 der Patienten zu korrelieren. Die Parkinson's Disease Sleep Scale hingegen weist starke Korrelationen mit dem Gesamtwert und den Unterrubriken der SF-36 auf.

\subsection{Ergebnisse Hypothese 4}

Die vierte Hypothese besagt, dass die Pflegebedürftigkeit der Parkinsonpatienten positiv mit dem Belastungserleben des betreuenden Angehörigen korreliere. Zur Überprüfung dieser Hypothese wurden die Korrelationen zwischen der NOSGER als Maß für die allgemeine Pflege- und Hilfsbedürftigkeit der Patienten und dem Fragebogen zur Belastung durch die Betreuung sowie dem SF-36 und der CESD-10 der betreuenden Kinder auf statistisch signifikante Zusammenhänge geprüft. Zudem wurde untersucht, inwiefern das Belastungserleben der Betreuer mit dem Gesundheitszustand des erkrankten Elternteils in Zusammenhang steht. Hierfür wurden die Korrelationen zwischen dem Belastungsbogen des betreuenden Kindes und dem SF-36 sowie den Depressionsfragebögen GDS nach Yesavage und CESD-10 überprüft. Darüber hinaus wurde die Abhängigkeit der Pflegebedürftigkeit (NOSGER) von Faktoren wie dem UPDRS-Motorscore, dem Krankheitsstadium nach Hoehn und Yahr, der Erkrankungsdauer und der Pflegestufe der Patienten untersucht. 


\subsubsection{NOSGER und Belastungserleben Betreuer}

Im Folgenden werden die Korrelationen der NOSGER mit dem Fragebogen zur Belastung durch die Betreuung, mit dem Fragebogen zur Gesundheitsbezogenen Lebensqualität (SF-36) sowie mit der CESD-10 der betreuenden Söhne und Töchter dargestellt.

Tabelle 32: Korrelationen der NOSGER mit dem Belastungsbogen, dem SF-36 und der CESD-10 der Betreuer

\begin{tabular}{|c|c|c|c|}
\hline \multicolumn{4}{|c|}{$\begin{array}{l}\text { Spearman's Rangkorrelation } \\
{ }^{\circ} \mathrm{p}<0,1 * \mathrm{p}<0,05 * *_{\mathrm{p}}<0,01 \quad * * * \mathrm{p}<0,001\end{array}$} \\
\hline & Söhne & Töchter & alle \\
\hline NOSGER \& Belastung & $* 0,57$ & $* * * 0,59$ & $* * * 0,58$ \\
\hline NOSGER \& KöFu (SF-36) & 0,23 & $-0,23$ & $-0,04$ \\
\hline NOSGER \& KöRo (SF-36) & 0,22 & $-0,06$ & 0,04 \\
\hline NOSGER \& KöSch (SF-36) & 0,24 & 0,02 & 0,11 \\
\hline NOSGER \& AlGes (SF-36) & 0,03 & $-0,29$ & $-0,18$ \\
\hline NOSGER \& Vital (SF-36) & 0,05 & $-0,29$ & $-0,16$ \\
\hline NOSGER \& SoFu (SF-36) & $-0,13$ & $* *-0,47$ & $*-0,35$ \\
\hline NOSGER \& EmRo (SF-36) & $-0,20$ & ${ }^{\circ}-0,34$ & $*_{-} 0,29$ \\
\hline NOSGER \& PsyWo (SF-36) & $-0,07$ & $-0,27$ & $-0,21$ \\
\hline NOSGER \& CESD-10 & 0,13 & 0,33 & 0,25 \\
\hline
\end{tabular}

Wie aus Tabelle 32 ersichtlich korreliert die NOSGER eindeutig statistisch signifikant mit dem Belastungserleben der betreuenden Kinder (Abbildung 61). Die positiven Korrelationen der Gesamtstichprobe $(\mathrm{r}=0,58 ; \mathrm{p}=0,000009)$ und der Töchter $(\mathrm{r}=0,59 ; \mathrm{p}=0,0005)$ erreichen das Signifikanzniveau von $\mathrm{p}<0,001$, die starke Korrelation der NOSGER mit dem Belastungsbogen der betreuenden Söhne das angestrebte Niveau von $\mathrm{p}<0,05(\mathrm{r}=0,57 ; \mathrm{p}=0,011)$.

Auf dem angestrebten Signifikanzniveau von $\mathrm{p}<0,05$ korreliert die NOSGER zudem negativ mit der „sozialen Funktionsfähigkeit“ ( $\mathrm{r}=-0,35 ; \mathrm{p}=0,013)$ und der „emotionalen Rollenfunktion“ ( $\mathrm{r}=-0,29$; $\mathrm{p}=0,04$ ) der betreuenden Kinder (Abbildungen 62 und 63). Der statistische Zusammenhang zwischen der NOSGER und der „sozialen Funktionsfähigkeit“ der betreuenden Töchter erreicht sogar das Signifikanzniveau von $\mathrm{p}<0,01(\mathrm{r}=-0,47 ; \mathrm{p}=0,008)$. Die negative Korrelation mit der ,emotionalen Rollenfunktion“ der Töchter ist hingegen mit $\mathrm{p}=0,06$ knapp nicht statistisch signifikant ( $\mathrm{r}=-0,34$; $\mathrm{p}=0,06)$. In der Gruppe der betreuenden Söhne lassen sich keine statistisch signifikanten Zusammenhänge zwischen der NOSGER und den Rubriken des SF-36 feststellen.

Die mäßig hoch positiven Korrelationen der NOSGER mit der CESD-10 in der Gruppe der Töchter $(\mathrm{r}=0,33 ; \mathrm{p}=0,07)$ und in der Gruppe der betreuenden Kinder insgesamt $(\mathrm{r}=0,25 ; \mathrm{p}=0,08)$ erreichen lediglich ein Signifikanzniveau von $\mathrm{p}<0,1$. Sie können daher allenfalls als Trend gewertet werden. 


\subsubsection{NOSGER und UPDRS, H\&Y, Erkrankungsdauer und Pflegestufe}

Tabelle 33 zeigt die Zusammenhänge zwischen der Ausprägung der Hilfsbedürftigkeit der Patienten (NOSGER) und den Faktoren, die eine objektive Aussage über den aufgrund der Parkinsonerkrankung beeinträchtigten Gesundheitszustand der Patienten zulassen, wie UPDRS-Motorscore, Hoehn-YahrStadium, Erkrankungsdauer und Pflegestufe.

Tabelle 33: Korrelationen der NOSGER mit dem UDPRS-Score, dem Hoehn-Yahr-Stadium, der Erkrankungsdauer und der Pflegestufe der Patienten

\begin{tabular}{|l|r|r|r|}
\hline $\begin{array}{l}\text { Spearman's Rangkorrelation } \\
* \mathrm{p}<0,05 * * \mathrm{p}<0,01 * * * \mathrm{p}<0,001\end{array}$ & \multicolumn{3}{|l|}{} \\
\hline & männl. Pat. & weibl. Pat. & Patienten insg. \\
\hline NOSGER \& UPDRS & 0,20 & $* * 0,58$ & $* * 0,43$ \\
\hline NOSGER \& H\&Y-Stadium & $* 0,41$ & $* 0,52$ & $* * * 0,47$ \\
\hline NOSGER \& Erkr.-Dauer & $* 0,47$ & $-0,19$ & 0,20 \\
\hline NOSGER \& Pflegestufe & $* * 0,48$ & $* 0,48$ & $* * * 0,48$ \\
\hline
\end{tabular}

Bezogen auf das gesamte Patientenkollektiv korreliert die NOSGER mittel hoch positiv mit dem UPDRS-Score $(r=0,43 ; \mathrm{p}=0,002)$ und stark hoch positiv mit dem Hoehn-Yahr-Stadium $(\mathrm{r}=0,47$; $\mathrm{p}=0,0006)$ und der Einstufung des Patienten in eine Pflegestufe $(r=0,48 ; p=0,0004)$. Bei den weiblichen Patientinnen steht das Maß der Hilfsbedürftigkeit auf einem Niveau von $p<0,01$ in statistisch signifikantem Zusammenhang mit dem UPDRS-Score ( $r=0,58 ; p=0,005)$, sowie auf dem angestrebten Signifikanzniveau von $\mathrm{p}<0,05$ in positivem Zusammenhang mit dem Hoehn-YahrStadium ( $\mathrm{r}=0,52 ; \mathrm{p}=0,012)$ und der Pflegestufe der Patientinnen $(\mathrm{r}=0,48 ; \mathrm{p}=0,02)$. Die NOSGER korreliert hingegen nicht signifikant mit der Erkrankungsdauer der Patientinnen. In der Gruppe der männlichen Patienten lassen sich positive signifikante Korrelationen mit dem Hoehn-Yahr-Stadium $(r=0,41 ; p=0,03)$, mit der Erkrankungsdauer $(r=0,47 ; p=0,0102)$ und der Pflegestufe $(r=0,48 ; p=0,009)$ der Patienten feststellen.

\subsubsection{Belastungsbogen und SF-36, GDS und CESD-10 (Patienten)}

In Tabelle 34 sind die Korrelationen des Belastungsbogens der Betreuer mit dem SF-36, der Geriatrischen Depressionsskala nach Yesavage (GDS) und der CESD-10 der Patienten dargestellt. 
Tabelle 34: Korrelationen des Belastungsbogens der Betreuer mit dem SF-36, der GDS und der CESD-10 der Patienten

\begin{tabular}{|c|c|c|c|}
\hline \multicolumn{4}{|c|}{$\begin{array}{l}\text { Spearman's Rangkorrelation } \\
{ }^{\circ} \mathrm{p}<0,1 * \mathrm{p}<0,05 * * \mathrm{p}<0,01 * * * \mathrm{p}<0,001\end{array}$} \\
\hline & Söhne & Töchter & alle \\
\hline Belastung \& KöFu (SF-36) & $*-0,48$ & $*_{-0,39}$ & $* *-0,45$ \\
\hline Belastung \& KöRo (SF-36) & $-0,14$ & $*_{-}-0,37$ & ${ }^{\circ}-0,27$ \\
\hline Belastung \& KöSch (SF-36) & $-0,34$ & $-0,31$ & $*_{-0,36}$ \\
\hline Belastung \& AlGes (SF-36) & $-0,44$ & $-0,26$ & $*_{-} 0,33$ \\
\hline Belastung \& Vital (SF-36) & $-0,03$ & $* * *-0,61$ & $* *-0,38$ \\
\hline Belastung \& SoFu (SF-36) & $-0,16$ & $*_{-}-0,45$ & $*_{-} 0,36$ \\
\hline Belastung \& EmRo (SF-36) & $-0,11$ & $-0,25$ & $-0,17$ \\
\hline Belastung \& PsyWo (SF-36) & 0,06 & $*_{-0,43}$ & $-0,24$ \\
\hline Belastung \& GDS & 0,13 & $* 0,40$ & $* 0,29$ \\
\hline Belastung \& CESD-10 & 0,21 & $* * 0,57$ & $* * 0,39$ \\
\hline
\end{tabular}

Während die Belastung der betreuenden Söhne auf dem angestrebten Niveau von $\mathrm{p}<0,05$ lediglich mit der „körperlichen Funktionsfähigkeit“ ( $r=-0,48 ; p=0,04)$ der erkrankten Elternteile signifikant negativ korreliert, lassen sich bei den Töchtern auf gleichem Signifikanzniveau negative Korrelationen mit der „körperlichen Funktionsfähigkeit“ ( $\mathrm{r}=-0,39 ; \mathrm{p}=0,03$ ), der „körperlichen Rollenfunktion“ ( $\mathrm{r}=-0,37$; $\mathrm{p}=0,04)$, der „,sozialen Funktionsfähigkeit“ ( $r=-0,45 ; \mathrm{p}=0,011)$ und dem ,psychischen Wohlbefinden“ $(\mathrm{r}=-0,43 ; \mathrm{p}=0,02)$ der betreuten Elternteile feststellen. Zudem steht die Belastung der Töchter statistisch eindeutig in negativem Zusammenhang mit der „Vitalität“ der Patienten ( $\mathrm{r}=-0,61$; $\mathrm{p}=0,0004)$. Für das Gesamt-Kollektiv der betreuenden Kinder ergeben sich zwischen dem Belastungsbogen und den folgenden Rubriken des elterlichen SF-36 statistisch signifikante negative Korrelationen: „körperliche Funktionsfähigkeit“ ( $r=-0,45$; $\mathrm{p}=0,0012)$, „körperliche Schmerzen“ ( $\mathrm{r}=-$ 0,36; $\mathrm{p}=0,011)$, ,allgemeine Gesundheit“ $(\mathrm{r}=-0,33 ; \mathrm{p}=0,02)$, „Vitalität“ $(\mathrm{r}=-0,38 ; \mathrm{p}=0,007)$ und „soziale Funktionsfähigkeit“ $(\mathrm{r}=-0,36 ; \quad \mathrm{p}=0,011)$. Der Zusammenhang zwischen dem Belastungserleben der betreuenden Angehörigen und der „körperlichen Rollenfunktion“ der Patienten $(\mathrm{r}=-0,27 ; \mathrm{p}=0,057)$ ist mit $\mathrm{p}=0,057$ nur knapp als nicht statistisch signifikant $\mathrm{zu}$ bezeichnen (Abbildungen 64-66).

Auf dem angestrebten Niveau von $p<0,05$ korreliert die Geriatrische Depressionsskala nach Yesavage der Patienten mäßig hoch positiv mit der Belastung der Töchter $(r=0,40 ; p=0,02)$ und der betreuenden Angehörigen insgesamt $(r=0,29 ; \mathrm{p}=0,04)$. Die positiven Korrelationen zwischen dem Belastungsbogen und der CESD-10 der erkrankten Elternteile erreichen bei den Töchtern ( $r=0,57 ; p=0,0011)$ und in der Studiengruppe insgesamt $(r=0,39 ; p=0,005)$ sogar das Signifikanzniveau von $\mathrm{p}<0,01$ (Abbildungen 67 und 68). Das Belastungserleben der Söhne steht weder mit der GDS noch mit der CESD-10 der Patienten in statistisch signifikantem Zusammenhang. 


\subsubsection{Belastungsbogen und UPDRS, H\&Y, Erkrankungsdauer und Pflegestufe}

Im Folgenden werden die Korrelationen zwischen dem Fragebogen zur Belastung durch die Betreuung und dem UPDRS-Motorscore, dem Hoehn-Yahr-Stadium der Erkrankungsdauer und der Einstufung der Patienten in eine Pflegestufe dargestellt und auf ihre statistische Signifikanz hin geprüft.

Tabelle 35: Korrelationen des Belastungsbogens der Betreuer mit dem UDPRS-Motorscore, dem Hoehn-Yahr-Stadium, der Erkrankungsdauer und der Pflegestufe der Patienten

\begin{tabular}{|l|r|r|r|}
\hline $\begin{array}{l}\text { Spearman's Rangkorrelation } \\
* \mathrm{p}<0,05 * * \mathrm{p}<0,01 * * * \mathrm{p}<0,001\end{array}$ & \multicolumn{3}{l|}{} \\
\hline & \multicolumn{1}{|l|}{ Söhne } & Töchter & alle \\
\hline Belastung \& UPDRS & $* 0,54$ & $* 0,44$ & $* * * 0,52$ \\
\hline Belastung \& H\&Y-Stadium & $* * 0,59$ & $* * 0,51$ & $* * * 0,55$ \\
\hline Belastung \& Erkr.-Dauer & 0,40 & 0,11 & 0,26 \\
\hline Belastung \& Pflegestufe & 0,40 & $* * 0,46$ & $* * 0,45$ \\
\hline
\end{tabular}

Der Belastungsbogen der betreuenden Angehörigen korreliert auf dem Niveau von $\mathrm{p}<0,001$ hoch signifikant mit dem UPDRS-Motorscore $(\mathrm{r}=0,52 ; \mathrm{p}=0,0002)$ und dem Erkrankungsstadium nach Hoehn \& Yahr ( $\mathrm{r}=0,55 ; \mathrm{p}=0,00004)$ der Patienten (Abbildungen 69 und 70). Der Zusammenhang zwischen Belastung des Betreuers und Pflegestufe des erkrankten Elternteils ist mit $p<0,01$ ebenfalls eindeutig signifikant ( $r=0,45 ; \mathrm{p}=0,0011)$, der Zusammenhang mit der Erkrankungsdauer des Patienten hingegen nicht $(r=0,26 ; p=0,07)$. Die positiven Korrelation des Belastungserlebens mit dem UDPRSScore und dem Hoehn-Yahr-Stadium des betreuten Elternteils ist bei den Söhnen (UPDRS: r=0,54; $\mathrm{p}=0,03 ; \mathrm{H} \& \mathrm{Y}: \mathrm{r}=0,59 ; \mathrm{p}=0,00096$ ) und Töchtern (UPDRS: $r=0,44 ; \mathrm{p}=0,02 ; \mathrm{H} \& \mathrm{Y}: \mathrm{r}=0,51 ; \mathrm{p}=0,004$ ) jeweils gleichermaßen statistisch signifikant. Die Belastung der Töchter korreliert zudem positiv mit der Pflegestufe der Patienten ( $r=0,46 ; p=0,0097)$. Weder für Töchter noch für Söhne besteht ein statistisch signifikanter Zusammenhang zwischen dem Belastungsbogen und der Erkrankungsdauer des betreuten Elternteils.

\subsubsection{Zusammenfassung Ergebnisse Hypothese 4}

Zusammenfassend bleibt festzustellen, dass die Pflegebedürftigkeit der erkrankten Elternteile in unterschiedlichem Ausmaß mit dem Belastungserleben der betreuenden Kinder in Zusammenhang steht. Die stärksten Korrelationen bestehen zwischen dem Grad der elterlichen Pflegebedürftigkeit und der subjektiven Belastung der erwachsenen Kinder und deren sozialen Faktoren der Gesundheitsbezogenen Lebensqualität. Zudem scheint ihr Belastungserleben stark mit dem Ausmaß der körperlichen Einschränkungen ihrer erkrankten Elternteile zusammenzuhängen. Hierbei sind die Korrelationen in der Gruppe der betreuenden Töchter wesentlich eindeutiger als bei den Söhnen. 


\subsection{Ergebnisse Hypothese 5}

Hypothese 5 lautet: Eine hohe filiale Reife und eine gute Schlafqualität korrelieren positiv miteinander. Um diese These zu belegen, wurden die Korrelationen zwischen den beiden Fragebögen zur Ausprägung der filialen Reife (Filial Anxiety Scale, Filiale-Reife-Bogen) und den Schlafbögen, Epworth Sleepiness Scale (ESS) und Pittsburgh Sleep Quality Index (PSQI), berechnet und auf ihre statistische Signifikanz hin untersucht.

Aufgrund der mit der relativ großen Anzahl an Unterskalen zusammenhängenden Fülle an Korrelationen, werden hier nur diejenigen statistischen Zusammenhänge dargestellt, die das angestrebte Signifikanzniveau von $\mathrm{p}<0,05$ erreichen oder dieses lediglich knapp verfehlen.

\subsubsection{Filial Anxiety Scale und ESS/PSQI}

Im Folgenden werden die Zusammenhänge zwischen der Filial Anxiety Scale (FAA/FAB) und der Epworth Sleepiness Scale (ESS) sowie des Pittsburgh Sleep Quality Index’ (PSQI) dargestellt.

Tabelle 36: Korrelationen der Filial Anxiety Scale mit der ESS und der PSQI der Betreuer

\begin{tabular}{|c|c|c|c|c|c|c|}
\hline \multicolumn{7}{|c|}{$\begin{array}{l}\text { Spearman's Rangkorrelation } \\
{ }^{\circ}<<0,1 * p<0,05 * * p<0,01 * * * p<0,001 \text { n.s. }=\text { nicht signifikant }\end{array}$} \\
\hline & \multicolumn{2}{|c|}{ Söhne } & \multicolumn{2}{|c|}{ Töchter } & \multicolumn{2}{|l|}{ alle } \\
\hline & FAA & FAB & FAA & FAB & FAA & FAB \\
\hline ESS & $-0,02$ & 0,04 & $-0,22$ & 0,09 & $-0,14$ & 0,13 \\
\hline PSQI gesamt & $-0,09$ & $-0,39$ & 0,23 & 0,28 & 0,12 & 0,08 \\
\hline subjekt. Schlafqualität & & & & & ${ }^{\circ} 0,28$ & \\
\hline Schlafdauer & & & & & ${ }^{\circ}-0,27$ & \\
\hline Schlafeffizienz & & & & $* 0,43$ & & \\
\hline Schlafstörungen & & & ${ }^{\circ} 0,34$ & & $* 0,32$ & $* 0,29$ \\
\hline
\end{tabular}

Auf dem angestrebten Signifikanzniveau von $\mathrm{p}<0,05$ korreliert weder die Skala A noch die Skala B der Filial Anxiety Scale mit der Epworth Sleepiness Scale der betreuenden Söhne und Töchter.

Zwischen der Filial Anxiety Scale und dem Gesamtwert des Pittsburgh Sleep Quality Index' lassen sich ebenfalls keine statistisch signifikanten Zusammenhänge belegen. Die FAA der betreuenden Angehörigen korreliert allerdings eindeutig positiv mit der PSQI-Unterskala Schlafstörungen $(r=0,32$; $\mathrm{p}=0,02)$. Die Korrelationen mit den Rubriken subjektive Schlafqualität $(\mathrm{r}=0,28 ; \mathrm{p}=0,051)$ und Schlafdauer $(r=-0,27 ; p=0,055)$ in der Gesamtstichprobe sowie den Schlafstörungen $(r=0,34 ; p=0,06)$ der Töchter verfehlen das statistisch signifikante Niveau von $\mathrm{p}<0,05$ hingegen knapp. Die FAB steht mit der Schlafeffizienz der Töchter $(r=0,43 ; p=0,02)$ und den Schlafstörungen der betreuenden Angehörigen $(r=0,29 ; p=0,04)$ in signifikant positivem Zusammenhang (Abbildungen 71 und 72). 


\subsubsection{Filiale-Reife-Bogen und ESS/PSQI}

Tabelle 37 zeigt die Zusammenhänge des Filiale-Reife-Bogens mit der Epworth Sleepiness Scale (ESS) und dem Pittsburgh Sleep Quality Index (PSQI).

Tabelle 37: Korrelationen des Filiale-Reife-Bogens mit der ESS und der PSQI der Betreuer

\begin{tabular}{|c|c|c|c|}
\hline \multicolumn{4}{|c|}{$\begin{array}{l}\text { Spearman's Rangkorrelation } \\
{ }^{\circ} \mathrm{p}<0,1 * \mathrm{p}<0,05 * *_{\mathrm{p}}<0,01 \quad * * * \mathrm{p}<0,001 \text { n.s. }=\text { nicht signifikant }\end{array}$} \\
\hline & Söhne & Töchter & alle \\
\hline Filiale-Reife-Bogen \& ESS & n.s. & n.s. & n.s. \\
\hline \multicolumn{4}{|l|}{ Filiale-Reife-Bogen \& PSQI } \\
\hline FO \& subj. Schlafqualität & & $* 0,36$ & \\
\hline FO \& Schlafeffizienz & $*-0,49$ & & \\
\hline FO \& Schlafstörungen & & & $* 0,31$ \\
\hline FH \& Schlafstörungen & & & ${ }^{\circ} 0,27$ \\
\hline FH \& Tagesschläfrigkeit & & & $* 0,34$ \\
\hline FS \& subj. Schlafqualität & & $*_{-0,38}$ & \\
\hline FS \& Schlafeffizienz & ${ }^{\circ}-0,45$ & & $*_{-} 0,29$ \\
\hline
\end{tabular}

Die Korrelationen zwischen den sieben Faktoren der filialen Reife und der ESS erreichen weder in der Gesamtstichprobe noch in den geschlechtsspezifisch getrennten Gruppen der betreuenden Söhne und Töchter das angestrebte Signifikanzniveau von $\mathrm{p}<0,05$.

Die Zusammenhänge mit dem Gesamtwert der PSQI bleiben ebenfalls in allen drei Gruppen statistisch nicht signifikant. Es kommt allerdings zu folgenden signifikant positiven Korrelationen zwischen den Rubriken des Filiale-Reife-Bogens und den Unterskalen der PSQI: zwischen dem „filialen Verpflichtungsgefühl“ und Schlafstörungen $(\mathrm{r}=0,31 ; \mathrm{p}=0,03)$ und zwischen dem Wert für „filiale Hilfe" und der Tagesschläfrigkeit $(r=0,34 ; p=0,014)$ in der Gesamtstichprobe sowie zwischen dem „filialen Verpflichtungsgefühl“ und der subjektiven Schlafqualität $(r=0,36 ; p=0,04)$ in der Gruppe der weiblichen Betreuer. Darüber hinaus korreliert die „familiäre Solidarität“" negativ mit der Schlafeffizienz der Gesamtstichprobe $(r=-0,29 ; \mathrm{p}=0,04)$ und der subjektiven Schlafqualität der Töchter $(r=-0,38 ; p=0,04)$. Bei den betreuenden Söhnen findet sich zudem eine negative Korrelation zwischen dem „filialen Verpflichtungsgefühl“ und der Schlafeffizienz $(r=-0,49 ; p=0,03)$. Die positiven statistischen Zusammenhänge der ,filialen Hilfe“ mit den Schlafstörungen der Betreuer ( $r=0,27$; $\mathrm{p}=0,057$ ) verpassen ebenso wie die negative Korrelation zwischen der ,familiären Solidarität“ und der Schlafeffizienz der Söhne ( $r=-0,45 ; \mathrm{p}=0,051)$ knapp das angestrebte Signifikanzniveau von $\mathrm{p}<0,05$.

\subsubsection{Zusammenfassung Ergebnisse Hypothese 5}

Zusammenfassend bleibt festzustellen, dass die Ausprägung der filialen Angst bzw. Reife der betreuenden Kinder insgesamt in einem eher schwachen Zusammenhang mit deren Tagesschläfrigkeit und allgemeiner Schlafqualität steht. Als wichtige Faktoren für die allgemeine Schlafqualität zeichnen sich vorrangig das „filiale Verpflichtungsgefühl“ und die „familiäre Solidarität“ ab. 


\section{Diskussion}

\subsection{Diskussion Fragebögen Patientengruppe}

\subsubsection{Anamnese, H\&Y, UPDRS, NOSGER}

Die große Übereinstimmung der demographischen Charakteristika (Alter, Geschlecht, Familien/Berufsstand) der an dieser Untersuchung teilnehmenden Parkinsonpatienten mit denen bisheriger Studienkollektive bildet die Grundlage für die Einschätzung der Ergebnisse hinsichtlich Lebensqualität, Schlafverhalten und der Prävalenz depressiver Symptome bei Parkinsonpatienten. Sowohl das durchschnittliche Patientenalter bei Datenerhebung (68,1 Jahre; SD=8,3), das mittlere Alter bei Erstdiagnose (59,6 Jahre; SD=10,2) als auch die Dauer der Parkinsonerkrankung (8,5 Jahre) in der aktuellen Stichprobe sind vergleichbar mit den Angaben verschiedener Autoren von weiteren Parkinsonstudien (vgl. Chaudhuri et al. 2002; Hagell et al. 2008; Happe und Berger 2002; Havlikova et al. 2008; Kuopio et al. 2000; Lökk 2008; Pal et al. 2004; Shpirer et al. 2006; Svensson et al. 2012; Weintraub et al. 2007). Auch bezüglich ihres Erkrankungsstadiums nach Hoehn und Yahr unterscheiden sich die Parkinsonpatienten der vorliegenden Untersuchung nicht wesentlich von anderen Studienpopulationen (Chaudhuri et al. 2002; Herlofson und Larsen 2003; Kuopio et al. 2000; Schrag et al. 2000; Shpirer et al. 2006). Mit einem mittleren Wert von 24 Punkten auf der UPDRSMotorskala sind sie zudem in Hinblick auf die Einschränkungen ihrer motorischen Fähigkeiten mit den Parkinsonpatienten der Studien von Svensson und Mitarbeitern, Herlofson und Larsen sowie Boddy und Kollegen vergleichbar (Boddy et al. 2007; Herlofson und Larsen 2003; Svensson et al. 2012). Die Untersuchungskollektive von Marras und Kollegen, Ferreira und Mitarbeitern sowie Muslimović und Kollegen weisen hingegen geringfügig bessere motorische Funktionen auf (Marras et al. 2008; Muslimović et al. 2008; Ferreira et al. 2006), während sowohl Merschdorf und Mitarbeiter als auch Havlikova et al. für ihre Studienteilnehmer deutlich stärkere Einschränkungen der motorischen Fähigkeiten angeben (Merschdorf et al. 2003; Havlikova et al. 2008).

Als weiterer Anhaltspunkt für die krankheitsbedingten Einschränkungen der Parkinsonpatienten und dem damit einhergehenden Betreuungsbedarf durch die erwachsenen Söhne und Töchter wurde in der vorliegenden Untersuchung die NOSGER eingesetzt (vgl. Kapitel 2.4.14). Im Vergleich mit den gesunden Eltern der Kontrollpersonen ergeben sich für die Parkinsonpatienten in allen Rubriken signifikant höhere NOSGER-Werte, was auf eine insgesamt deutlich schlechtere Alltagsfähigkeit der Parkinsonpatienten hinweist. Aufgrund dieser Tatsache können die Probanden der Kontrollgruppe klar von den betreuenden Söhnen und Töchtern abgegrenzt werden und können somit in dieser Untersuchung tatsächlich als deren Kontrollpersonen mit noch relativ gesunden, nicht hilfsbedürftigen Eltern fungieren.

Im Vergleich mit den in den Studien von Linkersdörfer und Stiens untersuchten Demenzpatienten sind die Parkinsonpatienten der aktuellen Untersuchung in ihrer Alltagsfähigkeit weniger eingeschränkt 
(vgl. Linkersdörfer 2006 und Stiens 2000). Ihre Hilfs- und Pflegebedürftigkeit fällt daher auch geringer aus. Anhalt hierfür bieten nicht nur die insbesondere im Vergleich mit den Probanden von Linkersdörfer wesentlich niedrigeren NOSGER-Werte der Parkinsonpatienten, sondern auch der Anteil der im Sinne der Pflegeversicherung als „pflegebedürftig“ eingestuften Studienteilnehmer. Während $81 \%$ der Demenzpatienten formal als pflegebedürftig gelten, betrifft dies in der aktuellen Untersuchung lediglich 15 Parkinsonpatienten (30\%). Der weitaus größte Anteil von $70 \%$ besitzt hier keine Pflegestufe. Deutlich unterscheiden sich die beiden Studienpopulationen zudem hinsichtlich ihres Durchschnittsalters, welches bei den Probanden von Linkersdörfer mit 83 Jahren weit über dem in dieser Untersuchung liegt (68,1 Jahre.).

\subsubsection{Geriatrische Depressionsskala nach Yesavage (GDS) und 10-Item Center for Epidemiological Studies Depression Scale (CESD-10)}

Die Auswertung der Ergebnisse der GDS und der CESD-10 ergaben bei den weiblichen Patientinnen einen Anteil von über dem Grenzwert für Depressivität liegenden Personen von jeweils knapp $60 \%$. Bei den Männern lagen $43 \%$ über dem Grenzwert der GDS und $50 \%$ über dem entsprechenden Grenzwert der CESD-10. Damit sind in der Patientengruppe dieser Untersuchung deutlich über die Hälfte der weiblichen Studienteilnehmerinnen und nahezu jeder zweite der männlichen Teilnehmer als depressiv verstimmt zu bezeichnen (entspr. 50 bzw. $54 \%$ in der Gesamtstichprobe).

In der entsprechenden Literatur variieren die Angaben zur Prävalenz depressiver Störungen bei Parkinsonpatienten teilweise erheblich. So lassen sich je nach diagnostischen Kriterien und Auswahl des Kollektivs Angaben zwischen vier und $70 \%$ finden. Im Mittel wird jedoch zumeist das Auftreten depressiver Symptome mit 40 \% angegeben (Khedr et al. 2013; Lemke 2008; Merschdorf et al. 2003; Poewe 2008; Quin et al. 2009; Slaughter et al. 2001).

Die Ergebnisse dieser Untersuchung sind vergleichbar mit denen von Meara und Mitarbeitern, in deren Studie der Anteil an Parkinsonpatienten mit GDS-Werten über dem cut-off für Depressivität bei $64 \%$ lag (Meara et al. 1999). Weintraub und Mitarbeiter, die in ihrer Studie mit 266 Parkinsonpatienten ebenfalls die 15-Fragen-Version der GDS einsetzen, kamen auf einen Depressivitätsanteil von $34 \%$ (Weintraub et al. 2007). Dieses Patientenkollektiv ist anhand Alter (mittl. Alter 68,2 Jahre) und kognitivem Status (mittl. MMST-Score 28,4 Punkte) mit der Stichprobe der aktuellen Untersuchung vergleichbar. Unterschiede ergeben sich jedoch hinsichtlich der Geschlechtsverteilung der Probanden. Während bei Weintraub et al. der Anteil männlicher Teilnehmer bei 69,3 \% lag, besteht die Patientengruppe dieser Studie nur zu $56 \%$ aus Männern. Da übereinstimmend mit bisherigen Forschungsergebnissen auch in dieser Studie der geschlechtsspezifische Unterschied in der Prävalenz der Depression zu Ungunsten der weiblichen Probandinnen verschoben ist (vgl. Andresen et al. 1994, Laux 2005), könnte dies die abweichenden Ergebnisse erklären. 
Bisherigen Forschungsergebnissen zufolge liegt die Prävalenz depressiver Symptome in der Allgemeinbevölkerung bei etwa zehn Prozent und steigt mit dem Alter leicht an (Andresen et al. 1994; Laux 2005; Lyness et al. 1997). Demnach leiden sowohl die weiblichen als auch die männlichen Parkinsonpatienten der aktuellen Untersuchung deutlich stärker unter depressiven Symptomen als altersentsprechende Referenzgruppen.

\subsubsection{Short-Form-36 Health Survey (SF-36)}

Im Vergleich mit den Daten des Bundes-Gesundheitssurveys von 1998 geben sowohl die männlichen als auch die weiblichen Parkinsonpatienten in allen acht Rubriken des SF-36 deutlich geringere Werte als die Probanden der Normstichprobe an (Ellert und Bellach 1999). Die gesundheitsbezogene Lebensqualität der Studienteilnehmer muss demnach als deutlich schlechter bewertet werden als die altersentsprechender Personen in der Normalbevölkerung. Besonders deutlich sind diese Unterschiede in den Rubriken „körperliche Funktionsfähigkeit“ sowie „körperliche“ und „emotionale Rollenfunktion“. Diese Ergebnisse stehen im Einklang mit bisherigen Forschungsergebnissen (Hagell et al. 2008; Kuopio et al. 2000; Riazi et al. 2003; Schrag et al. 2000). Riazi et al. fanden bei den Parkinsonpatienten ihrer Studie signifikant schlechtere Mittelwerte für alle SF-36-Rubriken als bei einer entsprechenden Referenzgruppe. Übereinstimmend mit den Ergebnissen der aktuellen Untersuchung waren die Unterschiede in den Bereichen „körperliche Funktionsfähigkeit“ sowie „körperliche“ und „emotionale Rollenfunktion“ besonders deutlich ausgeprägt. Insgesamt scheinen die Patienten der aktuellen Untersuchung in ihrer gesundheitsbezogenen Lebensqualität allerdings etwas weniger stark eingeschränkt zu sein als die Probanden von Riazi und Mitarbeitern (Riazi et al. 2003). Eine mit den aktuellen Ergebnissen vergleichbar starke Einschränkung beschreiben Hagell und Mitarbeiter in ihrer Studie zur Lebensqualität von Parkinsonpatienten (Hagell et al. 2008). In dieser Studie findet zwar kein direkter Vergleich der SF-36-Werte der Studiengruppe mit einer entsprechenden Referenzgruppe statt, da die Patientencharakteristika wie Durchschnittsalter, prozentualer Anteil männlicher und weiblicher Probanden am Gesamtkollektiv sowie mittlere Erkrankungsdauer jedoch eng mit denen der aktuellen Studie übereinstimmen, können die Ergebnisse von Hagell et al. gut mit denen der vorliegenden Untersuchung verglichen werden. Schrag et al. geben ebenfalls signifikant schlechtere Werte in den verschiedenen Bereichen der gesundheitsbezogenen Lebensqualität für die an ihrer Studie teilnehmenden Parkinsonpatienten im Vergleich mit altersentsprechenden gesunden Kontrollpersonen an (Schrag et al. 2000). Eine Ausnahme bildet hierbei die Rubrik „körperliche Schmerzen“, in der sich die Studienteilnehmer nicht signifikant von der Referenzgruppe unterscheiden. Während bei näherer Betrachtung der Untergruppe der 65-74jährigen Patienten im Vergleich mit der Studienpopulation der aktuellen Untersuchung das geringere Ausmaß körperlicher Schmerzen bei den Parkinsonpatienten von Schrag und Mitarbeitern auffällt, wird die „soziale Funktionsfähigkeit“ von den Patienten der vorliegenden Untersuchung als 
vergleichsweise deutlich besser angegeben. Die Ergebnisse der übrigen SF-36-Rubriken stimmen innerhalb gewisser Grenzen überein.

Die Geschlechtsunterschiede in der gesundheitsbezogenen Lebensqualität der aktuellen Untersuchung sind statistisch nicht signifikant und können daher nur als Trend gewertet werden. Auch fallen diese geschlechtsspezifisch unterschiedlichen Ergebnisse nicht einheitlich zu Gunsten oder Ungunsten des einen Geschlechts aus. In der internationalen Literatur konnten diesbezüglich bislang nur Kuopio und Mitarbeiter statistisch signifikante Unterschiede belegen (Kuopio et al. 2000). In fünf der acht Dimensionen gaben weibliche Patienten eine deutlich schlechtere Lebensqualität als die männlichen Studienteilnehmer an, in den drei verbleibenden Dimensionen (,,allgemeine Gesundheit“, „Vitalität“ und „emotionale Rollenfunktion“) kamen die weiblichen Teilnehmerinnen zwar ebenfalls auf vergleichsweise schlechtere Werte, die Unterschiede blieben hier jedoch statistisch nicht signifikant. Diese Geschlechtsunterschiede werden von Kuopio und Mitarbeitern mit dem gering höheren Durchschnittsalter und der längeren Krankheitsdauer der Frauen erklärt. Darüber hinaus lebten die weiblichen Probanden zu einem größeren Anteil alleine und gaben vergleichsweise häufiger Einsamkeit und eine stärkere Ausprägung depressiver Symptome an. Depression sei allgemein der mit einer verminderten Lebensqualität am stärksten assoziierte Faktor (Kuopio et al. 2000). Diese Funde können allerdings - wie bereits erwähnt - durch die Ergebnisse der aktuellen Untersuchung nicht bestätigt werden.

\subsubsection{Parkinson's Disease Sleep Scale (PDSS)}

Die für die vorliegende Studie befragten Parkinsonpatienten zeigen einen mit den Ergebnissen von Chaudhuri vergleichbaren Gesamtwert in der PDSS und beurteilen somit ihre allgemeine Schlafqualität als in einem vergleichbaren Maße eingeschränkt wie die Parkinsonpatienten und als signifikant schlechter als die gesunden Kontrollpersonen der Originalstudie (Chaudhuri et al. 2002). Chaudhuri und Mitarbeiter stellten zudem einen Zusammenhang zwischen fortgeschrittenem Erkrankungsstadium und reduzierter Schlafqualität fest. So fielen die PDSS-Gesamtwerte bei Patienten der Hoehn-Yahr-Stadien 4 und 5 deutlich niedriger als die der Patienten in den Stadien 1 bis 3 aus. Diese Ergebnisse weichen von denen der aktuellen Untersuchung ab, durch die keine statistisch signifikante Korrelation zwischen beiden Faktoren bestätigt werden kann. Aufgrund der sehr geringen Anzahl von nur acht Patienten mit einem fortgeschrittenen Erkrankungsstadium (Hoehn \& Yahr Stadien 4 und 5) sollte diese fehlende Signifikanz jedoch nicht überbewertet werden. Bisherige Forschungsergebnisse belegen zudem eindeutig eine Zunahme von Frequenz und Ausprägung von Schlafstörungen bei Parkinsonpatienten mit Fortschreiten der Erkrankung (Comella 2003 und 2007; Happe und Mayer 2006; Lees et al. 1988; Poewe 2008). Svensson und Mitarbeiter wiederum konnten in ihrer Studie zu Schlafstörungen bei Parkinsonpatienten ebenfalls keinen Zusammenhang zwischen einem Fortschreiten der Erkrankung und einer Zunahme der berichteten Schlafstörungen feststellen (Svensson et al. 2012). Die an ihrer Studie teilnehmenden Parkinsonpatienten sind nicht nur anhand 
der Geschlechtsverteilung der Teilnehmer, des durchschnittlichen Erkrankungsstadiums nach Hoehn \& Yahr sowie dem mittleren UPDRS-motor-score sondern insbesondere auch anhand ihres ermittelten PDSS-Gesamtwertes von 102,8 (SD 23,3) [verglichen mit 99,6 (SD 24,3)] sehr gut mit der Studienpopulation der vorliegenden Untersuchung vergleichbar. Signifikante positive Korrelationen bestanden den Angaben von Svensson und Kollegen zufolge jedoch zwischen dem Grad der Schlafstörungen und der SF-36-Rubrik „Psychisches Wohlbefinden“ der Patienten. Zudem war eine schlechte Schlafqualität mit dem Vorhandensein von „,fatigue“-Symptomen sowie der Komorbidität eines Restless-legs-Syndroms assoziiert (Svensson et al. 2012).

Eine ausführlichere Diskussion weiterer die Schlafqualität der Parkinsonpatienten beeinflussender Faktoren findet in den Kapiteln 4.1.5, 4.1.6 und 4.6 statt.

\subsubsection{Epworth Sleepiness Scale (ESS)}

Sowohl M.W. Johns, der Erstautor der ESS, als auch Bloch und Mitarbeiter, die die deutsche Version des Fragebogens zuerst beschrieben, setzten die Epworth Sleepiness Scale bei einer Gruppe von Probanden mit Schlafstörungen und daneben auch bei einer Gruppe gesunder Kontrollpersonen ein (Bloch et al. 1999; Johns 1991). Im Vergleich mit den Ergebnissen dieser beiden Studien liegt das durchschnittliche Ausmaß der Tagesschläfrigkeit der Parkinsonpatienten in der vorliegenden Untersuchung etwa mittig zwischen dem der Probanden mit Schlafstörungen und dem der gesunden Kontrollpersonen. Die Aussagekraft dieses Vergleichs wird allerdings dadurch eingeschränkt, dass sowohl die Probanden von Johns und Bloch et al. als auch deren gesunde Referenzgruppen ein deutlich geringeres Durchschnittsalter als die Parkinsonpatienten der aktuellen Studie aufweisen

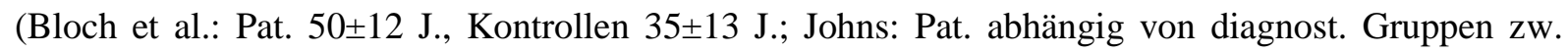

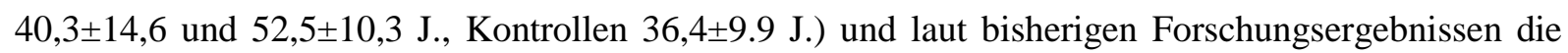
Tagesmüdigkeit generell auch in der Normalbevölkerung mit dem Alter ansteigt (Happe 2003).

Shpirer und Mitarbeiter definieren in ihrer Studie Tagesschläfrigkeit ab einem Wert von >10 Punkten in der ESS als exzessiv und kommen so auf einen Anteil von 50\% unter exzessiver Tagesschläfrigkeit leidender Parkinsonpatienten (Shpirer et al. 2006). Bei gleichem Maßstab betrifft dies in der aktuellen Untersuchung 21 von 47 Patienten, also in etwa 45 Prozent. Die Tagesschläfrigkeit der altersentsprechenden Kontrollpersonen von Shpirer et al. war signifikant geringer ausgeprägt. Entsprechend berichten auch Boddy et al. und Ferreira et al. über signifikant höhere Prävalenzen abnormaler Tagesschläfrigkeit bei Parkinsonpatienten (41\% bzw. 33,5\%) im Vergleich zu altersentsprechenden Referenzgruppen (10\% bzw. 16,1\%) (Boddy et al. 2007; Ferreira et al. 2006). Im Gegensatz hierzu fanden Tandberg und Mitarbeiter exzessive Tagesschläfrigkeit nur bei 15,5 Prozent der an ihrer Studie teilnehmenden Parkinsonpatienten. Bei weiteren 11,3 Prozent der Patienten wurde eine milde Tagesschläfrigkeit festgestellt (Tandberg et al. 1999). Ein Erklärungsversuch für diese von der bisherigen Literatur sowie der aktuellen Untersuchung stark abweichenden Ergebnisse könnte das 
grundsätzlich verschiedene Studiendesign sein. Während in den bereits diskutierten Studien als Maß für exzessive Tagesschläfrigkeit ein Wert von über zehn Punkten in der Epworth Sleepiness Scale diente und damit lediglich die Wahrscheinlichkeit, in den angegebenen Situation einzunicken, angegeben wurde, werden die unterschiedlichen Kategorien der Tagesschläfrigkeit von Tandberg et al. anhand der tatsächlichen Einschlafhäufigkeit und Schlafdauer der Parkinsonpatienten während des Tages abgestuft (keine: 1-2x Einschlafen, Gesamtschlafdauer <1h; milde: 2x Einschlafen, Gesamtschlafdauer 1-2h; exzessive: $\geq 3 x$ Einschlafen oder Gesamtschlafdauer $\geq 2 h$ ). Da diese Kriterien insgesamt als vergleichsweise strenger zu bewerten sind, ist davon auszugehen, dass mit diesem Maßstab auch in den Studienpopulationen von Boddy et al., Ferreira et al. und Shpirer et al. sowie in der aktuellen Untersuchung geringere Prävalenzen an exzessiver Tagesschläfrigkeit gemessen werden würden. Dies könnte zumindest einen Teil der Diskrepanz zwischen den voneinander abweichenden Ergebnissen der vorgenannten Studien erklären.

Weiterhin besteht Uneinigkeit hinsichtlich prädiktiver Faktoren für das Ausmaß exzessiver Tagesschläfrigkeit bei Parkinsonpatienten. Während Boddy et al. und Shpirer et al. keinen Zusammenhang zwischen dem Auftreten exzessiver Tagesschläfrigkeit und Erkrankungsdauer und schwere sowie depressiven Symptomen und beginnendem kognitiven Abbau feststellen konnten (Boddy et al. 2007; Shpirer et al. 2006), berichten Tandberg und Kollegen, dass ihren Untersuchungen zufolge ein erhöhtes Ausmaß exzessiver Tagesschläfrigkeit signifikant mit einem höheren Erkrankungsstadium, schwereren körperlichen Einschränkungen, vermehrten depressiven Symptomen, schwächerer kognitiver Funktion und längerer L-Dopa-Therapiedauer verbunden sei. Patientenalter, Erkrankungsdauer und Höhe der L-Dopa-Dosis seien keine prädiktiven Faktoren (Tandberg et al. 1999). Ondo et al. fanden hingegen deutliche Korrelationen mit einer längeren Erkrankungsdauer und -schwere (Ondo et al. 2001). Ferreira und Mitarbeiter konnten zwar keine Assoziationen zwischen gestörter nächtlicher Schlafqualität und erhöhter Tagesmüdigkeit bei Parkinsonpatienten feststellen, sehen jedoch einen deutlichen Zusammenhang zwischen erhöhten ESS-Werten und dem gehäuften Auftreten sogenannter „sleep attacks“ (Ferreira et al. 2006). Aufgrund der geringen Gruppengröße können in der vorliegenden Studie keine Aussagen bezüglich prädiktiver Faktoren gemacht werden. Insgesamt verdeutlichen die dargestellten unterschiedlichen Untersuchungsergebnisse jedoch gut die multifaktorielle Genese exzessiver Tagesschläfrigkeit bei Parkinsonpatienten.

\subsubsection{Pittsburgh Sleep Quality Index (PSQI)}

Im Vergleich mit den Referenzwerten von Buysse et al. weisen die Parkinsonpatienten der vorliegenden Studie eine allgemein deutlich schlechtere Schlafqualität als die gesunden Kontrollpersonen auf. Dies gilt sowohl für den PSQI-Gesamtwert als auch für die sieben Unterrubriken des Index „subjektive Schlafqualität“, „Schlaflatenz“, „Schlafdauer“, „Schlafeffizienz“, „Schlafstörungen“, „Schlafmittelkonsum“ und „Tagesmüdigkeit“. Die Parkinsonpatienten liegen damit allerdings noch besser als die beiden Gruppen „schlechter Schläfer“ von Buysse und 
Mitarbeitern mit Depression bzw. klinisch relevanten Ein- und Durchschlafstörungen (Buysse et al. 1989).

Boddy et al. berichten in ihrer Studie zur Schlafqualität von Parkinson- und Demenzpatienten von mit den aktuellen Ergebnissen vergleichbaren PSQI-Gesamtwerten ihrer nicht dementen Parkinsonpatienten (Boddy et al. 2007). Diese Studienpopulation unterscheidet sich allerdings durch ein um sieben Jahre höheres Durchschnittsalter, eine halb so lange durchschnittliche Erkrankungsdauer sowie eine Überrepräsentation des männlichen Geschlechts am Gesamtkollektiv (75\%) von den Probanden der vorliegenden Untersuchung. Ferreira und Mitarbeiter stellen bei den an ihrer Untersuchung teilnehmenden Parkinsonpatienten im Vergleich mit einer Gruppe altersentsprechender gesunder Kontrollpersonen eine signifikant schlechtere Schlafqualität fest (Ferreira et al. 2006). Insbesondere die Frequenz nächtlichen Aufwachens und nächtlicher Toilettengänge sowie die Prävalenz von Schnarchen, Beinkrämpfen, unruhigen Beinen, Albträumen und Halluzinationen während der Nacht sei im Patientenkollektiv deutlich erhöht. Auch in der Studie von Khedr und Kollegen werden Schlafstörungen von rund $79 \%$ der teilnehmenden Parkinsonpatienten angegeben. Ein- und Durchschlafstörungen wurden hierbei mit $46 \%$ am häufigsten genannt, lebhafte und bedrohliche Albträume zu $28 \%$ (Khedr et al. 2013). Die Ergebnisse der aktuellen Untersuchung deuten auf eine im Vergleich mit diesen Daten bessere Schlafqualität der teilnehmenden Patienten hin. Während Ferreira et al. einen Anteil von 65,3 \% „schlechter Schläfer“ angeben, betrifft dies in der aktuellen Untersuchung lediglich $52 \%$. Da die Patienten von Ferreira und Mitarbeitern bei vergleichbarem Durchschnittsalter eine längere Erkrankungsdauer als die Patienten dieser Studie aufweisen, könnte dies aufgrund der Tatsache, dass eine fortgeschrittene Parkinsonerkrankung mit stärker ausgeprägten Schlafstörungen und somit auch mit einer stärker reduzierten Schlafqualität assoziiert ist (Happe und Mayer 2006), die abweichenden Ergebnisse zumindest teilweise erklären.

\subsection{Diskussion Fragebögen Betreuergruppe und Kontrollgruppe}

\subsubsection{Anamnese}

Auch in dieser Untersuchung war der Großteil der Personen, die sich in der Betreuung ihres an dem idiopathischen Parkinsonsyndrom erkrankten Elternteils engagierten, weiblich (vgl. Brody 1985; Tesch-Römer 2001). Der Anteil betreuender Söhne lag mit $38 \%$ allerdings deutlich über den in der bisherigen Literatur zu pflegenden Angehörigen beschriebenen Angaben von zehn bis zwölf Prozent (vgl. Daire 2002). Mit einem Durchschnittsalter von 38 Jahren (22-58 Jahre) waren die betreuenden Söhne und Töchter deutlich jünger als die pflegenden Kinder der Demenzpatienten in den Vorstudien von Linkersdörfer und Stiens, die ein mittleres Alter von 54 bzw. 53 Jahren aufwiesen (Linkersdörfer 2006; Stiens 2000). Der in verschiedenen Forschungsarbeiten beschriebene Trend, dass sich erwachsene Kinder eher im mittleren bis höheren als im jüngeren bis mittleren Erwachsenenalter 
befinden, wenn sie sich aufgrund einer chronischen Krankheit der Eltern mit deren drohender Pflegebedürftigkeit konfrontiert sehen, trifft auf die aktuelle Stichprobe demnach nicht zu. Auch die Tatsache, dass 40 Prozent der betreuenden Söhne und Töchter ledig waren und fast die Hälfte keine eigenen Kinder hatten, entspricht nicht der Erwartung, dass die Pflege hilfsbedürftiger Familienmitglieder in die späte Familienphase der Pflegenden fällt, in der die eigenen Kinder bereits erwachsen sind und das Elternhaus verlassen haben (vgl. Brody 1981; Linkersdörfer 2006). Eine Erklärung hierfür ist eventuell im Studiendesign der aktuellen Untersuchung zu finden. Da das Manifestationsalter des idiopathischen Parkinsonsyndroms in der sechsten Lebensdekade und somit deutlich unter dem der Alzheimerdemenz liegt (Schulz und Gasser 2007), ist folglich davon auszugehen, dass nicht nur die Parkinsonpatienten der aktuellen Untersuchung sondern auch deren betreuende erwachsene Söhne und Töchter wesentlich jünger sind als die Alzheimerpatienten bzw. deren pflegende Kinder in den Vorstudien von Linkersdörfer und Stiens (Linkersdörfer 2006; Stiens 2000). In diesem Zusammenhang sei auch noch einmal darauf hingewiesen, dass es sich bei den erwachsenen Kindern der Parkinsonpatienten nicht um aktiv pflegende Familienangehörige wie in den Vorstudien von Linkersdörfer und Stiens handelt sondern um betreuende erwachsene Kinder, welche sich um die Belange des erkrankten Elternteils kümmern (vgl. Kapitel 2.1).

Bemerkenswert ist der relativ geringe Anteil hauptsächlich betreuender Söhne und Töchter von lediglich einem Drittel der Gesamtstichprobe. In der Mehrzahl der Fälle oblag die Funktion des Hauptbetreuers dem Partner des erkrankten Elternteils. Dieser Befund findet Bestätigung bei Lowenstein, dem zufolge die Hauptquelle der Unterstützung der jeweilige Lebenspartner des Hilfsbedürftigen ist, und diese Rolle zumeist erst bei Abwesenheit eines/einer jenen den erwachsenen Kindern der hilfsbedürftigen Person zufällt (Lowenstein 1999).

Lediglich sechs der hauptsächlich und nicht-hauptsächlich betreuenden erwachsenen Kinder gaben an, in der Betreuung des erkrankten Elternteils keine Unterstïtzung von Dritten (Lebenspartner, eigene Kinder, nicht-erkranktes Elternteil, o.ä.) zu bekommen. Daher stimmt auch diese Untersuchung mit bisherigen Forschungsergebnissen überein, laut denen die Pflege eines chronisch kranken Familienmitglieds primär als „Familienaufgabe“ anzusehen ist (vgl. Brody 1981 und 1985; Schütze 1995; Tesch-Römer 2001).

Aufgrund der Auswahlkriterien für die Kontrollpersonen der betreuenden Söhne und Töchter besteht kein Unterschied zwischen diesen beiden Teilnehmergruppen hinsichtlich Durchschnittsalter, Geschlechtsverteilung und Bildungs- bzw. Berufsstand (vgl. Kapitel 2.1). Auch bezüglich objektiver Gesundheitsparameter wie Anzahl der Co-Morbiditäten und regelmäßig eingenommener Medikamente sind die Stichproben gut miteinander vergleichbar. Es bestand allerdings eine Tendenz in der Kontrollgruppe, im Vergleich mit der Betreuergruppe mehr eigene Kinder zu haben, häufiger auf dem Land zu leben und geringfügig jüngere Eltern zu haben. Darüber hinaus lag die Frequenz der monatlichen Kontakte zu den Eltern bei den betreuenden Söhnen und Töchtern etwas höher, die 
räumliche Entfernung zum elterlichen Wohnort war etwas niedriger als bei den Kontrollpersonen. Letztere Unterschiede sind wahrscheinlich auf die Methode zur Rekrutierung der Probanden zurückzuführen. Während die betreuenden Söhne und Töchter von ihren erkrankten Elternteilen jeweils als dasjenige Kind hervorgehoben wurden, das sich in gesundheitlichen Belangen des erkrankten Elternteils im Vergleich zu den Geschwistern am meisten engagierte - was häufig mit der Kontaktfrequenz und der räumlichen Distanz in Zusammenhang stand - und daraufhin bei ihnen um Mitarbeit an der vorliegenden Untersuchung geworben wurde, wurden die Kontrollpersonen ohne vorherige Involvierung der Eltern direkt angesprochen. Hierbei war eher von Bedeutung, dass bei ihnen ein regelmäßiger Kontakt zu den Eltern bestand, und nicht, ob es sich bei ihnen um dasjenige Kind innerhalb der Geschwisterreihe handelte, welches zu den Eltern die engste Verbindung pflegte.

\subsubsection{Filial Anxiety Scale (FAS)}

Im Vergleich mit Cicirellis Referenzgruppe lassen die für diese Untersuchung befragten betreuenden Söhne und Töchter vergleichbare Werte für filiale Angst erkennen (Cicirelli 1988). Sie unterscheiden sich somit weder im Ausmaß ihrer Sorge bezüglich der eigenen Fähigkeit bei der Übernahme der Betreuungs-/Pflegerolle (filiale Angst A) noch bezüglich der Sorge um das Wohlergehen der alternden Eltern (filiale Angst B) von der Studienpopulation von Cicirelli, die sich aus Männern und Frauen mittleren Alters mit noch unabhängig lebenden und relativ gesunden Eltern zusammensetzt. Dies ist dahingehend bemerkenswert, da sich die betreuenden Kinder in ihrer filialen Angst durchaus signifikant von den für diese Studie untersuchten Kontrollpersonen mit nicht-hilfsbedürftigen Eltern unterscheiden. So empfinden die betreuenden Söhne eine deutlich größere Sorge bezüglich ihrer eigenen Fähigkeit, die Pflegerolle für das erkrankte Elternteil zu übernehmen, als die männlichen Kontrollpersonen, und die betreuenden Töchter sind signifikant stärker um das Wohlergehen der alternden Eltern besorgt als die weiblichen Kontrollpersonen ohne erkranktes Elternteil. Letzteres ist kein unerwartetes Ergebnis, da es in Anbetracht einer bestehenden chronisch-progressiven Erkrankung eines Elternteils nahe liegend erscheint, sich als erwachsenes Kind vermehrt um die Gesundheit des betroffenen Elternteils zu sorgen. Zudem stimmt der Befund mit bisherigen Forschungsergebnissen überein (Cicirelli 1988). Das unterschiedliche Ausmaß an Sorge bezüglich der eigenen Fähigkeit zur Pflegeübernahme von betreuenden Söhnen und männlichen Kontrollpersonen kann im Zusammenhang mit Blenkners Begriff der „filialen Krise“ gesehen werden (Blenkner 1965). Im Gegensatz zu den Kontrollpersonen mit gesunden Eltern sehen sich die betreuenden Söhne mit der Parkinsonerkrankung und damit verbunden auch mit einem sich nun verschlechternden Gesundheitszustand des Elternteils sowie einer dadurch möglicherweise auf sie zukommenden Pflegesituation konfrontiert. Durch die Erkenntnis, dass ihre Eltern in Zukunft verstärkt auf ihre Hilfe angewiesen sein werden, geraten sie in die filiale Krise. Die Auseinandersetzung mit der eigenen Fähigkeit, einer solchen Betreuungssituation gerecht werden zu können, wird angestoßen. Naheliegend erscheint demnach, dass die betreuenden Söhne der aktuellen Untersuchung ein größeres Ausmaß an Sorge empfinden als die Kontrollpersonen, 
die sich aufgrund des noch relativ guten Gesundheitszustandes der eigenen Eltern in naher Zukunft nicht mit einer vergleichbaren Situation konfrontiert sehen. Dies steht auch im Einklang mit Ergebnissen von Laditka und Pappas-Rogich, die eine vergleichsweise höhere Sorge bezüglich einer zukünftigen Pflegeübernahme bei Personen, die schon einmal in der Pflege eines Angehörigen involviert waren, als bei Personen, die noch nie in solch einer Position fungiert hatten, feststellten (Laditka und Pappas-Rogich 2001). Hierfür spricht ebenfalls, dass sich in der vorliegenden Untersuchung ein deutlicher Trend bezüglich einer größeren filialen Angst hauptsächlich betreuender Kinder im Vergleich zu den nicht hauptsächlich betreuenden Kindern abzeichnet, welche bezüglich der Untereinheit filiale Angst B statistische Signifikanz erreicht. Die weitere Erkenntnis aus der Studie von Laditka und Pappas-Rogich, dass Frauen grundsätzlich ein höheres Maß an Sorge zeigen als Männer, konnte durch die aktuelle Untersuchung allerdings nicht bestätigt werden.

\subsubsection{Louvain Filial Maturity Scale A (LFMS-A)}

Wie in den Untersuchungen von Marcoen ergeben sich in der vorliegenden Studie in der Ausprägung der filialen Reife teilweise wesentliche Geschlechtsunterschiede (Marcoen 1993 und 1995). So zeigen die betreuenden Töchter entsprechend Marcoens Ergebnissen ein signifikant höheres „filiales Verpflichtungsgefühl“ und eine signifikant höhere „filiale Hilfsbereitschaft“. Darüber hinaus unterscheiden sich die betreuenden Töchter der aktuellen Studienpopulation signifikant durch ihre größere „filiale Hilfe“ von den betreuenden Söhnen. Da die Pflege von Familienangehörigen traditionell der Rolle der Frau zufiel und auch in der heutigen Gesellschaft ein diesbezügliches Engagement noch immer eher von Töchtern als von Söhnen erwartet wird (vgl. Schütze und Wagner 1991), überraschen diese Ergebnisse nicht. Demgegenüber zeigen die betreuenden Söhne ebenso wie die männlichen Studienteilnehmer bei Marcoen eine größere „filiale Autonomie“ und schätzen zudem auch die „elterliche Besorgnis“ höher ein als die betreuenden Töchter. Auch diese geschlechtsspezifischen Unterschiede sind nicht unerwartet, da die Tendenz, eine gewisse Distanz zwischen sich und die Eltern zu bringen und sich so vor möglichen Wünschen und Forderungen ein Stück weit zu schützen, allgemein bei Männern stärker ausgeprägt ist als bei Frauen - und Eltern vermutlich wiederum aufgrund bestehender Rollenverständnisse ein solches Verhalten tendenziell auch eher den Söhnen als den Töchtern zubilligen.

Erwähnenswert erscheinen in der Diskussion um den Grad der filialen Reife der untersuchten Stichprobe auch die Unterschiede in den Ergebnissen zwischen hauptsächlich und nicht-hauptsächlich betreuenden Töchtern und Söhnen. Zwar wird diesbezüglich das in dieser Untersuchung angestrebte Signifikanzniveau verfehlt, doch zeigt sich bei den hauptsächlich betreuenden erwachsenen Kindern eine klare Tendenz für höhere Werte in den Rubriken „filiale Liebe“ und „filiales Verpflichtungsgefühl“ sowie in der „filialen Hilfsbereitschaft“, „filialen Hilfe“ und „familiären Solidarität“" als bei den nicht-hauptsächlich betreuenden Söhnen und Töchtern. Ob die hauptsächlich betreuenden Kinder nun aufgrund ihrer vergleichsweise höheren filialen Liebe ein höheres 
Verpflichtungsgefühl und damit auch eine größere Hilfsbereitschaft gegenüber ihren alternden Eltern empfinden und sich aufgrund dessen in der Betreuung des erkrankten Elternteils als Hauptansprechpartner fühlen, oder ob diese betreuenden Söhne und Töchter aus Gründen der Notwendigkeit zu Hauptansprechpartnern in gesundheitlichen Belangen für ihre erkrankten Elternteile werden und aus dieser Position heraus ein vergleichsweise höheres filiales Verpflichtungsgefühl sowie eine größere Hilfsbereitschaft und filiale Hilfe entwickeln, lässt sich allerdings durch die aktuelle Datenlage nicht näher eruieren. Hier könnten zukünftige Untersuchungen zum Entwicklungsstadium der filialen Reife ansetzen.

Die im Vergleich mit den Ergebnissen der Vorstudie von Linkersdörfer deutlich höheren Werte für „filiale Liebe“ und „elterliche Besorgnis“ in der aktuellen Untersuchung könnten mitunter auf die unterschiedlichen Charakteristika der Stichproben zurückzuführen sein (vgl. Linkersdörfer 2006). Bei den Probanden von Linkersdörfer handelt es sich um erwachsene Kinder demenzkranker Eltern, deren allgemeine kognitive Funktionen aufgrund ihrer vorangeschrittenen Demenz sehr viel stärker eingeschränkt sind als die der an dieser Untersuchung teilnehmenden Parkinsonpatienten. So erscheint es nicht verwunderlich, dass der Grad der elterlichen Besorgnis von den Probanden der aktuellen Studie als höher empfunden wird als von den Probanden der Voruntersuchung, deren Eltern krankheitsbedingt nicht mehr so viel Rücksicht auf die pflegerische Leistungsfähigkeit ihrer erwachsenen Kinder nehmen können. Damit verbunden könnte auch der höhere Grad filialer Liebe in der aktuellen Stichprobe erklärt werden: In einer erwachsenen Eltern-Kind-Beziehung, die auf Gegenseitigkeit und Rücksichtnahme (elterliche Besorgnis) beruht, und in der sich das Kind daher von den Eltern akzeptiert und verstanden fühlt, erscheint viel mehr Raum für positive Gefühle von Seiten des Kindes gegenüber den Eltern (filiale Liebe) möglich zu sein als in Beziehungen, in der das erwachsene Kind die elterliche Besorgnis und somit das elterliche Verständnis für die eigene Person als gering empfindet. Diese Hypothese bedarf allerdings weiterer Untersuchungen.

Da ein vergleichsweise sehr hohes filiales Verpflichtungsgefühl sowie eine uneingeschränkte filiale Hilfsbereitschaft und Hilfe eher Indikatoren für ein filial unreifes Verhalten darstellen, können die betreuenden Söhne und Töchter der teilnehmenden Parkinsonpatienten aufgrund geringerer Werte in diesen drei Rubriken im Vergleich mit den Probanden von Linkersdörfer als insgesamt filial reifer bzw. als sich nicht unmittelbar in der filialen Krise befindend bezeichnet werden. Die in der aktuellen Stichprobe festgestellte größere filiale Autonomie, die nach Ansicht verschiedener Autoren einen wesentlichen Aspekt filialer Reife darstellt (Adler et al. 1996; Bruder 1988), ist ein weiterer Hinweis hierfür. Unklar bleibt in diesem Zusammenhang hingegen der Einfluss des krankheitsbedingten Abhängigkeitsgrads der Eltern auf die vorbenannten Faktoren der filialen Reife. So ist es den Probanden von Linkersdörfer aufgrund der vorangeschrittenen elterlichen Demenzerkrankung eventuell gar nicht möglich, ein hohes $\mathrm{Maß}$ an filialer Autonomie gegenüber den Eltern zu wahren, da filiale Autonomie sich nicht nur auf die Unabhängigkeit der Kinder von ihren Eltern bezieht sondern 
auch eine Aussage darüber beinhaltet, inwiefern sich die Kinder in das Leben der Eltern einmischen. Ebenso könnte eine vergleichsweise größere Hilfsbedürftigkeit der Eltern auch ein größeres filiales Verpflichtungsgefühl sowie ein größeres $\mathrm{Maß}$ an tatsächlich geleisteter Hilfe von Seiten des Kindes einfordern.

Die von den betreuenden Söhnen im Vergleich mit den männlichen Kontrollpersonen der aktuellen Untersuchung als signifikant geringer empfundene „familiäre Solidarität“ steht im Einklang mit bisherigen Forschungsergebnissen, denen zufolge eine zunehmende Pflegebelastung im Verlauf der Erkrankung der Eltern vermehrt zu Unstimmigkeiten, Vorwürfen und Schuldzuweisungen innerhalb der Ursprungsfamilie führt und somit der innerfamiliäre Zusammenhalt abnimmt (Brody et al. 1989; Linkersdörfer 2006). Die im Vergleich mit den weiblichen Kontrollpersonen signifikant höhere „filiale Hilfe“ der betreuenden Töchter ist wohl ebenso im Zusammenhang mit dem Erkrankungsstadium und der damit verbundenen größeren Hilfsbedürftigkeit der Eltern zu sehen. Da es sich bei den Kontrollpersonen um erwachsene Kinder vergleichsweise noch relativ gesunder Eltern handelt, besteht in diesen Eltern-Kind-Beziehungen ein geringerer Bedarf an elterlicher Unterstützung und so auch ein geringeres $\mathrm{Maß}$ an tatsächlich geleisteter Hilfe als in den Beziehungen zwischen den betreuenden Töchtern und ihren am Parkinsonsyndrom erkrankten Elternteilen.

\subsubsection{Fragebogen zur Belastung durch die Betreuung}

Im Einklang mit bisherigen Forschungsergebnissen empfinden auch die für diese Untersuchung befragten Töchter die Belastung durch die Betreuung des erkrankten Elternteils im Vergleich mit den betreuenden Söhnen als stärker (Cicirelli 1993; Faison et al. 1999; Linkersdörfer 2006; MartinezMartin et al. 2007; Raschick und Ingersoll-Dayton 2004; Schütze und Wagner 1991; Yee und Schulz 2000). Schütze und Wagner führen das geringere Belastungserleben der Söhne in der Pflege alternder Eltern auf die ihnen gegenüber von Seiten der Gesellschaft entgegengebrachte geringere Erwartungshaltung und dem damit verbundenen geringeren Erwartungsdruck zurück. So werde die Gefahr der Entwicklung von Schuldgefühlen verringert und das Wohlbefinden der betreuenden Söhne weniger beeinträchtigt (Schütze und Wagner 1991). Faison et al. diskutieren darüber hinaus die vergleichsweise höhere Wahrscheinlichkeit für verheiratete bzw. in einer festen Partnerschaft lebenden Männer, durch ihre Ehefrauen/ Partnerinnen in der Pflege ihrer alternden Eltern unterstützt zu werden, als mögliche Erklärung für das durch betreuende Söhne geringer empfundene Belastungserleben (Faison et al. 1999). Cicirelli stellt die stärkere Belastung der betreuenden Töchter in den Zusammenhang mit dem durch sie geleisteten größeren und konstanteren Ausmaß an elterlicher Unterstützung und Hilfe (Cicirelli 1993). Verschiedenen Autoren zufolge ist ein allgemein höheres Belastungsempfinden pflegender Angehöriger mit dem vermehrten Auftreten physischer und psychischer Symptomen assoziiert (Chappell und Reid 2002; Etters et al. 2008; Happe und Berger 2002; Kurz et al. 2003; Lökk 2008; Vitaliano et al. 2004). In diese Richtung weisende Ergebnisse 
finden sich auch in dieser Untersuchung und werden in den Kapiteln 4.2.5 und 4.2.6 eingehender behandelt.

Das signifikant höhere Belastungserleben der hauptsächlich betreuenden Kinder im Vergleich mit den nicht-hauptsächlich betreuenden Söhnen und Töchtern steht ebenfalls im Einklang mit bisherigen Forschungsergebnissen, denen zufolge ein eindeutiger Zusammenhang zwischen dem Ausmaß der geleisteten Unterstützung und der Höhe der empfundenen Belastung des pflegenden Angehörigen besteht (Faison et al. 1999; Happe und Berger 2002; Miyashita et al. 2009; Savla et al. 2008; Vitaliano et al. 2004). Chappell und Reid sehen das Belastungserleben pflegender Angehöriger zudem durch die Pausenfrequenz und die Stundenanzahl der informellen Pflege beeinflusst (Chappell und Reid 2002).

Insgesamt zeigen die betreuenden Söhne und Töchter der aktuellen Untersuchung eine wesentlich geringere Belastung durch die Betreuung als die Probanden der Vorstudien von Stiens und Linkersdörfer (vgl. Linkersdörfer 2006 und Stiens 2000). Da unter anderem Thommessen et al. in ihrer Studie zu Unterschieden im Belastungserleben betreuender Angehörigen von Parkinson-, Alzheimer- und Schlaganfallpatienten keine wesentlichen Unterschiede bezüglich der psychosozialen Belastung in den drei Krankheitsgruppen feststellen konnten (Thommessen et al. 2002), kann wohl davon ausgegangen werden, dass die im Vergleich mit den Vorstudien als weniger stark empfundene Belastung der Probanden der aktuellen Stichprobe nicht auf die unterschiedlichen Krankheiten der betreuten Elternteile (Demenz vom Alzheimertyp versus idiopathisches Parkinsonsyndrom) sondern auf weitere Unterschiede in den Charakteristika der Studienpopulationen zurückzuführen ist. So sind die zu betreuenden Eltern in den Voruntersuchungen aufgrund ihrer Demenz wesentlich stärker in ihrer Eigenständigkeit (NOSGER) eingeschränkt als die Parkinsonpatienten der vorliegenden Studie, wie bereits in Kapitel 4.1.1 erwähnt. Die Pflegebedürftigkeit der demenzkranken Eltern ist demnach als vergleichsweise deutlich höher einzuschätzen. So erscheint es nicht verwunderlich, dass die betreuenden Kinder der Studien von Stiens und Linkersdörfer ihre Belastung durch die Betreuung auch als wesentlich stärker als die erwachsenen Söhne und Töchter der aktuellen Untersuchung empfinden. Da verschiedenen Studien zufolge eine Verbindung zwischen der subjektiven Pflegebelastung und dem Alter des pflegenden Angehörigen besteht (Kim et al. 2007; Vitaliano et al. 2004), könnte das in den beiden Vorstudien um 15 Jahre höhere Durchschnittsalter der betreuenden Söhne und Töchter ebenfalls einen Einfluss auf die hier als stärker empfundene Belastung haben.

\subsubsection{Short-Form-36 Health Survey (SF-36)}

In der internationalen Literatur wurde der Erfassung der Lebensqualität betreuender Angehöriger hilfsbedürftiger älterer Personen besonders in den letzten Jahrzehnten vermehrt Aufmerksamkeit geschenkt - mit allerdings teilweise uneinheitlichen Forschungsergebnissen. So weisen beispielsweise Yee und Schulz in einer Überblicksarbeit darauf hin, dass Frauen aufgrund der Betreuung alternder 
Familienangehöriger im Vergleich zu Männern neben einer erhöhten allgemeinen psychiatrischen Morbidität auch eine stärker eingeschränkte Lebensqualität zeigen (Yee und Schulz 2000), während verschiedenen anderen Autoren zufolge diesbezüglich keine Geschlechtsunterschiede bestehen (vgl. Glozman 2004; Martínez-Martín et al. 2007; O’Rourke et al. 2003; Schrag et al. 2006; Zhan 2005). Im Einklang hiermit können auch in der aktuellen Stichprobe keine signifikanten Unterschiede in der gesundheitsbezogenen Lebensqualität zwischen betreuenden Töchtern und Söhnen festgestellt werden. Die erwachsenen Söhne zeigen lediglich eine Tendenz hin zu einer besseren Einschätzung ihrer körperlichen Rollenfunktion und Vitalität.

Unerwartet und daher bemerkenswert ist, dass die betreuenden erwachsenen Kinder dieser Untersuchung eine vergleichbare bzw. in bestimmten Rubriken sogar bessere gesundheitsbezogene Lebensqualität angeben als die altersentsprechende Normstichprobe des Bundes-Gesundheitssurveys von 1998. Dies steht im Gegensatz zu der allgemein gültigen These, dass sich die Pflege hilfsbedürftiger Angehöriger negativ auf die physische und psychische Gesundheit der Pflegenden auswirkt und somit ihr Wohlbefinden und ihre Lebensqualität beeinträchtigt (vgl. Etters et al. 2008; Faison et al. 1999; Glozman 2004; Lökk 2008; Martínez-Martín et al. 2008; Pinquart und Sörensen 2003; Schrag et al. 2006). So schätzen laut einer Studie von Faison und Mitarbeitern etwa $91 \%$ der betreuenden Kinder chronisch kranker Eltern rückblickend ihren Gesundheitszustand zum Zeitpunkt vor Pflegeübernahme als exzellent oder gut ein, wohingegen dies im Verlauf der Pflegebeziehung nur noch auf 74\% der Pflegenden zutrifft (Faison et al. 1999). O'Rourke et al. zufolge ist eine negative subjektive Gesundheitsbewertung mit einer erhöhten psychologischen Belastung der betreuenden Angehörigen im Verlauf der Pflegebeziehung verbunden (O’Rourke et al. 2003). Auch MartínezMartín und Mitarbeiter sowie Kurz und Kollegen sehen einen eindeutigen Zusammenhang zwischen der Belastung durch die Betreuung und der gesundheitsbezogenen Lebensqualität - insbesondere den psychosozialen und mentalen Komponenten - der pflegenden Angehörigen von Parkinsonpatienten (Kurz et al. 2003; Martínez-Martín et al. 2007). Im Einklang hiermit lässt sich auch in der vorliegenden Untersuchung für die betreuenden Söhne und Töchter bezüglich ihrer „sozialen Funktionsfähigkeit“ eine signifikant schlechtere Lebensqualität feststellen als bei den eigens für diese Untersuchung befragten alters- und geschlechtsentsprechenden Kontrollpersonen. Zudem können folgende (nicht-signifikante) Trends als in die gleiche Richtung weisend bewertet werden: Die betreuenden Töchter zeigen eine deutlich schlechtere „körperliche Rollenfunktion“ als die weiblichen Kontrollpersonen und die betreuenden Söhne schätzen ihre gesundheitsbezogene Lebensqualität in den Rubriken „emotionale Rollenfunktion“ und „psychisches Wohlbefinden“ sowie bei den „körperlichen Schmerzen“ und der ,allgemeinen Gesundheit“ im Vergleich mit den männlichen Kontrollpersonen als niedriger ein. Diese Ergebnisse relativieren in gewisser Hinsicht auch die unerwartet fehlende Diskrepanz in puncto Lebensqualität zwischen den betreuenden erwachsenen Kindern der vorliegenden Untersuchung und der Normstichprobe des Bundes-Gesundheitssurveys von 1998. Während es sich bei den Probanden des Bundes-Gesundheitssurveys lediglich um einen 
repräsentativen Querschnitt durch die Gesellschaft handelt, liegt die Übereinstimmung wesentlicher Charakteristika zwischen Betreuer- und Kontrollgruppe in der aktuellen Untersuchung aufgrund des Studiendesigns deutlich höher (vgl. Kapitel 2.1 und 4.2.1). Entsprechenden Vergleichen kommt somit ebenfalls eine deutlich höhere Aussagekraft zu.

\subsubsection{0-Item Center for Epidemiological Studies Depression Scale (CESD-10)}

Der in dieser Stichprobe bei $18 \%$ liegende Anteil von depressiv verstimmten betreuenden Söhnen und Töchter steht im Einklang mit bisherigen Forschungsergebnissen, denen zufolge die Prävalenz depressiver Symptome bei pflegenden Personen hilfsbedürftiger Familienmitgliedern zwischen neun und $46 \%$ liegt und mit dem prozentualen Anteil von betreuenden Ehepartnern und weiblichen Pflegenden am Gesamtkollektiv ansteigt (Kurz et al. 2003; Martínez-Martín et al. 2008; Meara et al. 1999; Miyashita et al. 2009; Molyneux et al. 2008; Raveis et al. 1998; Schrag et al. 2004). Entsprechend den aktuellen Ergebnissen stellten Schrag und Mitarbeiter bei etwa $20 \%$ der von ihnen befragten jugendlichen und erwachsenen Kindern von Parkinsonpatienten milde bis moderate Level an depressiver Symptomatik fest (Schrag et al. 2004). Laut der Studie von Raveis et al. leiden etwa $30 \%$ der pflegenden Töchter von Krebspatienten unter Depressionen, die in direktem Zusammenhang mit ihrer subjektiven Pflegebelastung stehen (Raveis et al. 1998). Auch Caap-Ahlgren und Dehlin sowie Martínez-Martín und Mitarbeiter berichten von einem negativen Einfluss depressiver Symptome auf die allgemeine Belastung durch die Betreuung von Parkinsonpatienten (Caap-Ahlgren und Dehlin 2002; Martínez-Martín et al. 2007). Hinweise hierfür finden sich auch in der vorliegenden Untersuchung. Phillips et al. stellten dabei nicht nur einen Zusammenhang zwischen einer stärkeren Pflegebelastung und einer erhöhten Prävalenz an Depressionen sondern auch einen Zusammenhang zwischen einem geringen sozialen Rückhalt und dem vermehrten Auftreten depressiver und Angstsymptome bei pflegenden Personen fest (Phillips et al. 2009). Darüber hinaus ist einer Studie von Zhan zufolge eine subjektiv als schlechter empfundene Gesundheit relevant für das erhöhte Auftreten von Depressionen bei betreuenden Kindern und Schwiegerkindern alternder Menschen (Zhan 2005).

Die in der Literatur diskutierte vergleichsweise höhere Prävalenz depressiver Symptome bei weiblichen pflegenden Angehörigen (vgl. Amirkhanyan und Wolf 2006; Yee und Schulz 2000) wird auch durch die Ergebnisse der vorliegenden Untersuchung bestätigt. So liegen aktuell acht betreuende Töchter aber nur ein betreuender Sohn mit ihren CESD-10-Werten über dem von Andresen et al. vorgegebenen cut-off für Depressivität (vgl. Andresen et al. 1994). Die - entgegen den Erwartungen fehlende Signifikanz im Grad der Depressivität von hauptsächlich und nicht-hauptsächlich betreuenden Söhnen und Töchtern könnte im Zusammenhang mit Ergebnissen von Amirkhanyan und Wolf gesehen werden, laut denen nicht nur pflegende Angehörige sondern auch nicht-pflegende Kinder und Schwiegerkinder hilfsbedürftiger älterer Menschen (sogenannte „noncaregiver“) vermehrt unter depressiven Symptomen leiden als „noncaregiver“ nicht-hilfsbedürftiger Eltern (Amirkhanyan 
und Wolf 2006). Die Autoren stellten daher die Hypothese auf, dass nicht die Pflege der alternden Eltern an sich sondern viel mehr deren Pflegebedürftigkeit ausschlaggebend für die Depressivität ihrer erwachsenen Kinder sei.

Überraschend ist die Tatsache, dass sich die betreuenden erwachsenen Kinder in der vorliegenden Untersuchung in ihrer Depressivität nicht signifikant von den alters- und geschlechtsspezifischen Kontrollpersonen unterscheiden, was im Gegensatz zu bisherigen Forschungsergebnissen steht (vgl. Etters et al. 2008; Martínez-Martín et al. 2008; Pinquart und Sörensen 2003; Schulz et al. 1995; Yee und Schulz 2000). Die betreuenden Söhne der aktuellen Erhebung geben zwar im Vergleich mit den männlichen Kontrollpersonen vermehrt depressive Symptome an, dies kann aufgrund der fehlenden Signifikanz jedoch lediglich als Trend gewertet werden. Der CESD-10-Mittelwert der betreuenden Töchter liegt hingegen deutlich - wenn auch nicht signifikant - unter dem der weiblichen Kontrollpersonen. Demnach sind in der aktuellen Untersuchung die weiblichen Kontrollpersonen depressiver als die betreuenden Töchter, welche ihrerseits depressiver als die betreuenden Söhne und diese wiederum depressiver als die männlichen Kontrollpersonen sind. Dem gegenüber stehen Ergebnisse von Yee und Schulz, denen zufolge sich der negative Einfluss der Pflegebelastung bei Frauen deutlich stärker auf den Grad ihrer Depressivität auswirkt als bei Männern (Yee und Schulz 2000). Pinquart und Sörensen kamen in ihrer Meta-Analyse zu Unterschieden zwischen pflegenden und nicht-pflegenden Personen ebenfalls zu dem Schluss, dass pflegende Angehörige alternder Menschen allgemein unter einem vergleichsweise höheren Ausmaß an Stress und Depressionen sowie einem verminderten subjektiven Wohlbefinden und einer schlechteren körperlichen Gesundheit leiden. Im speziellen Hinblick auf pflegende Angehörige nicht-dementer älterer Erwachsener ließen sich signifikante Unterschiede jedoch lediglich für die Größen „Stress“, „,subjektives Wohlbefinden“ sowie „körperliche Gesundheit“ und nicht in Bezug auf das Ausmaß ihrer allgemeinen Depressivität feststellen. Auch seien die Unterschiede bezüglich der Stress- und Depressions-Level zwischen pflegenden und nicht-pflegenden Angehörigen in Stichproben mit einem höheren prozentualen Anteil an betreuenden (Ehe-) Partnern deutlich größer als in Studienkollektiven mit vorwiegend oder ausschließlich betreuenden erwachsenen Kindern wie in der aktuellen Untersuchung (Pinquart und Sörensen 2003). Weitere Unterschiede bestehen in der Art und dem Umfang der geleisteten Pflege: Im Gegensatz zu den Probanden der vorliegenden Untersuchung handelt es sich beispielsweise in den Studien der Meta-Analyse von Pinquart und Sörensen um betreuende Angehörige, welche zu $71 \%$ mit der pflegebedürftigen Person zusammen in einem Haushalt lebten und pro Woche etwa 43 Stunden mit der Pflege ihres Angehörigen verbrachten (vgl. Pinquart und Sörensen 2003). Die betreuenden erwachsenen Kinder der vorliegenden Untersuchung teilen jedoch in aller Regel nicht den Haushalt mit ihrem erkrankten Elternteil. Auch nehmen sie ihnen gegenüber keine aktiv pflegende Rolle ein, sondern stehen ihrem Elternteil eher beratend-betreuend zur Seite. So ist die unerwartet niedrige Rate 
an Depressivität unter den erwachsenen betreuenden Kindern möglicherweise in diesem Rahmen zu erklären.

\subsubsection{Epworth Sleepiness Scale (ESS) und Pittsburgh Sleep Quality Index (PSQI)}

Die im Vergleich mit den betreuenden Söhnen stärkere Tagesschläfrigkeit und schlechtere allgemeine Schlafqualität der betreuenden Töchter verfehlen zwar jeweils das angestrebte statistische Signifikanzniveau, weisen als Trend jedoch in die gleiche Richtung wie bisherige Forschungsergebnisse, denen zufolge Frauen allgemein mehr Schlafstörungen und eine stärker beeinträchtigte Schlafqualität aufweisen als Männer (vgl. Buysse et al. 2008; Smith et al. 1997; Zeitlhofer et al. 2000). Diesen Ergebnissen kritisch gegenüber stehen hingegen Voderholzer und Kollegen, die Geschlechtsunterschiede in der Prävalenz von Schlafstörungen weder bei Insomnie-Patienten noch bei gesunden erwachsenen Kontrollpersonen feststellen konnten (Voderholzer et al. 2003). Sie kamen daher zu der Schlussfolgerung, dass die vielfach in der internationalen Literatur berichtete höhere Prävalenz von Schlafstörungen bei Frauen nicht allein auf ihr Geschlecht zurückzuführen sei, sondern eher im Zusammenhang mit der bei Frauen ebenfalls höheren Prävalenz von Angststörungen und Depressionen verstanden werden müsse, da diese psychiatrischen Störungen die Schlafqualität der Betroffenen per se negativ beeinflusse (vgl. hierzu Beaudreau et al. 2008; Buysse et al. 2008; Fava 2004; Franzen und Buysse 2008). Yoshioka und Kollegen wiesen ebenfalls nach, dass die höhere Prävalenz von Schlafstörungen bei Frauen im Allgemeinen nicht auf ihr Geschlecht sondern auf geschlechtsspezifische Unterschiede zwischen Männern und Frauen bezüglich den an sie gerichteten beruflichen und familiären Anforderungen zurückzuführen ist (Yoshioka et al. 2012). Ihrer Untersuchung zufolge bestand lediglich in der Gruppe von Probanden, welche vergleichsweise günstige Arbeits- und Lebensumstände angaben (keine Schichtarbeit, wenig Bildschirmarbeit, wenig beruflicher Erfolgsdruck, geringer Zeitaufwand für Haushaltstätigkeiten, kein Zusammenleben mit einem Pflegebedürftigen), ein tatsächlich erhöhtes Risiko für Frauen, unter Schlafstörungen zu leiden. Die betreuenden Söhne und Töchter der aktuellen Untersuchung zeigen sowohl bezüglich ihrer Tagesschläfrigkeit als auch bezüglich ihrer allgemeinen Schlafqualität vergleichbare Werte wie die gesunden Referenzgruppen von Bloch und Mitarbeitern beziehungsweise Zeitlhofer und Kollegen (Bloch et al. 1999; Zeitlhofer et al. 2000). Auch im Vergleich mit den alters- und geschlechtsentsprechenden Kontrollpersonen der aktuellen Untersuchung sind keine wesentlichen Unterschiede festzustellen. In punkto allgemeine Schlafqualität sind zwar insgesamt weniger Kontrollpersonen als betreuende erwachsene Kinder als „schlechte Schläfer“ zu bezeichnen, aufgrund der fehlenden Signifikanz kann dies aber ebenfalls nur als Trend gewertet werden. Diese unerwarteten Ergebnisse stehen im deutlichen Gegensatz zu Veröffentlichungen zahlreicher Autoren, die bei pflegenden Angehörigen signifikant stärkere Einschränkungen in der Schlafqualität als bei nicht-pflegenden 
Personen gleichen Alters beschreiben (Happe und Berger 2002; Lökk 2008; McKibbin et al. 2005; Rowe et al. 2008; Smith et al. 1997; Wilcox und King 1999; Willette-Murphy et al. 2006). In einer Studie von Pal und Mitarbeitern lag der Anteil „schlechter Schläfer“ (PSQI-Gesamtwert >5) bei pflegenden Ehepartnern von Parkinsonpatienten beispielsweise bei 40 Prozent, während 17 Prozent unter exzessiver Tagesschläfrigkeit litten ( $\mathrm{Pal}$ et al. 2004). Entsprechend berichten Smith und Kollegen von regelmäßigen Schlafstörungen bei 27 Prozent der betreuenden Ehemänner und bei 48 Prozent der betreuenden Ehefrauen von Parkinsonpatienten (Smith et al. 1997). Da sich die Literatur zu Schlafstörungen bei pflegenden Angehörigen in den meisten Fällen auf die Ehepartner der pflegebedürftigen Personen bezieht, es sich bei den betreuenden Angehörigen in der vorliegenden Untersuchung nun aber um die erwachsenen Kinder von Parkinsonpatienten und nicht um deren Ehepartner handelt, könnte dies als Erklärungsansatz für die unerwartet niedrige Prävalenz von Tagesschläfrigkeit und Schlafstörungen in der aktuellen Stichprobe dienen. Verschiedenen Studien zufolge lassen sich Schlafstörungen pflegender Angehöriger häufig auf notwendige Hilfeleistungen (z.B. bei Toilettengängen) sowie auf nächtliches Störverhalten der zu Pflegenden zurückführen (Creese et al. 2008; McCurry et al. 2004). Die betreuenden erwachsenen Kinder der vorliegenden Untersuchung teilen jedoch in aller Regel nicht den Haushalt mit ihrem erkrankten Elternteil, aufgrund dessen dieser negative Einfluss auf ihren Schlaf auch entfällt. Unterstützung findet dieser Erklärungsansatz bei Wilcox und King, die in ihrer Studie mit weiblichen pflegenden Angehörigen von Demenzpatienten, die in über 92 Prozent der Fälle mit der pflegebedürftigen Person zusammenlebten, keinen Unterschied bezüglich der Schwere von Schlafstörungen zwischen betreuenden Ehefrauen und anderen weiblichen Familienangehörigen nachweisen konnten (Wilcox und King 1999). Auch Beaudreau et al. stellten in ihrem Studienkollektiv, das zu 53\% aus betreuenden Ehefrauen und zu 38\% aus betreuenden Töchtern von Demenzpatienten bestand und die in vier von fünf Fällen auch mit diesem zusammenlebten, keinen Zusammenhang zwischen der allgemeinen Schlafqualität und der Art der Beziehung zum zu pflegenden Familienmitglied fest (Beaudreau et al. 2008). Uneinigkeit besteht bei den Autoren allerdings hinsichtlich des Einflusses des Alters der pflegenden Person auf ihre Schlafqualität. Während laut Wilcox und King das Alter der pflegenden Personen in keiner signifikanten Relation zu deren Schlafqualität steht (Wilcox und King 1999), wiesen Beaudreau und Kollegen den betreffenden Zusammenhang eindeutig nach (Beaudreau et al. 2008). Da das mittlere Alter der betreuenden Söhne und Töchter der aktuellen Untersuchung bei 38 Jahren und somit weit unter dem Durchschnittsalter der in der Pflegeforschung üblichen Studienkollektive liegt (vgl. Beaudreau et al. 2008; Creese et al. 2008; Happe und Berger 2002; Lökk 2008; Rowe et al. 2008; Wilcox und King 1999; Willette-Murphy et al. 2006), sollte auch das jüngere Alter der Probanden als mögliche Erklärung für die vergleichsweise geringe Prävalenz übermäßiger Tagesschläfrigkeit und reduzierter Schlafqualität in der vorliegenden Untersuchung in Betracht gezogen werden. 
Im Gegensatz zu den bereits diskutierten Forschungsergebnissen - und somit im Einklang mit denen der aktuellen Untersuchung - konnten Brummett und Kollegen keinen direkten negativen Einfluss der Pflege hilfsbedürftiger Familienangehöriger auf das Schlafverhalten der Pflegenden belegen (Brummett et al. 2006). Kochar und Mitarbeiter fanden ebenfalls bei pflegenden Angehörigen und nicht-pflegenden gleichaltrigen Personen keine Unterschiede in Art und Ausmaß bestehender Schlafprobleme (Kochar et al. 2007). Sie führen aufgrund ihrer Studienergebnisse das Auftreten von Schlafstörungen eher auf das Vorhandensein depressiver Symptome zurück. So bestand für stark depressiv verstimmte pflegende Angehörige das größte Risiko für Schlafstörungen, gefolgt von depressiven nicht-pflegenden Personen. Zwischen nicht depressiven pflegenden Angehörigen und nicht depressiven nicht-pflegenden Personen bestand kein Unterschied in der Prävalenz von Schlafstörungen. Auch verschiedenen weiteren Autoren zufolge führt eine depressive Symptomatik zu einer Verschlimmerung der bestehenden Schlafstörungen pflegender Angehöriger (Beaudreau et al. 2008; Creese et al. 2008; Pal et al. 2004; Phillips et al. 2009; Smith et al. 1997; Wilcox und King 1999). Anhand der Ergebnisse der aktuellen Untersuchung können ebenfalls in die gleiche Richtung weisende Korrelationen festgestellt werden. So ist zwar die Tagesschläfrigkeit der betreuenden erwachsenen Kinder nicht signifikant mit dem Ausmaß ihrer Depressivität verbunden, doch besteht insbesondere bei den betreuenden Töchtern ein eindeutiger Zusammenhang zwischen dem vermehrten Auftreten depressiver Symptome und der Reduktion ihrer allgemeinen Schlafqualität.

\subsection{Diskussion Hypothese 1}

Sowohl die Korrelationen zwischen der Filial Anxiety Scale und den drei das Belastungserleben der betreuenden Kinder widerspiegelnden Fragebögen als auch die Korrelationen zwischen dem FilialeReife-Bogen und den drei das Belastungserleben widerspiegelnden Fragebögen bestätigen die erste Hypothese, nach der die Ausprägung der filialen Angst bzw. der filialen Reife in engem Zusammenhang mit dem Ausmaß der subjektiv empfundenen Pflegebelastung der erwachsenen Söhne und Töchter steht.

Eine insgesamt höhere filiale Angst geht den Ergebnissen dieser Untersuchung zufolge mit einem erhöhten Belastungserleben einher. In der Einzelbetrachtung der beiden Rubriken der filialen Angst wird deutlich, dass eine verhältnismäßig große Sorge der erwachsenen Kinder über die eigene Fähigkeit, der erwarteten Hilfsbedürftigkeit der alternden Eltern entsprechen zu können (filiale Angst A, gemessen durch die FAA), eng mit einer hohen subjektiven Belastung der betreuenden Kinder verbunden ist. Die entsprechende Korrelation verfehlt zwar knapp das geforderte statistische Signifikanzniveau, der Trend weist mit $\mathrm{p}=0,0505$ jedoch eindeutig in diese Richtung.

Ergebnissen der aktuellen Untersuchung zufolge steigt die filiale Angst A zudem mit dem Fortschreiten der elterlichen Erkrankung (Stadium nach Hoehn und Yahr) und der Zunahme der 
elterlichen Beeinträchtigungen (NOSGER). Darüber hinaus steht in der Gruppe der betreuenden Töchter eine verhältnismäßig große Sorge bezüglich der eigenen Fähigkeiten, der erwarteten Rolle als pflegende Person gerecht $\mathrm{zu}$ werden, in engem Zusammenhang mit einer Einschränkung ihrer emotionalen Rollenfunktion sowie mit einer erhöhten Prävalenz depressiver Symptome.

Bezüglich des kausalen Zusammenhangs zwischen der filialen Angst $\mathrm{A}$ und dem Belastungsempfinden kann aufgrund des Studiendesigns nur spekuliert werden: Denkbar wäre einerseits, dass die steigende Sorge der betreuenden Kinder bezüglich ihrer eigenen Fähigkeit zu einer angemessenen elterlichen Pflege Auslöser für ein erhöhtes Belastungserleben ist. Es kann weiter vermutet werden, dass auch der Wunsch der pflegenden Angehörigen nach Erfüllung der an sie gerichteten gesellschaftlichen und familiären Erwartungen bezüglich der Pflege-Übernahme den Druck auf die Pflegenden erhöht, in dessen Folge ein verstärktes Belastungserleben mit psychischen und physischen Symptomen ausgelöst werden kann (vgl. Schmidt 2009). Andererseits könnten sich die betreuenden Kinder aber auch erst aufgrund ihrer zunehmenden subjektiven Pflegebelastung gedanklich vermehrt mit ihren diesbezüglichen Fähigkeiten und Grenzen auseinander setzen. Unter Berücksichtigung der aktuellen elterlichen Beeinträchtigungen könnte dies wiederum zu einer Zunahme der Sorge der betreuenden Kinder über ihre eigenen Fähigkeiten führen, auch der zukünftig erwarteten Hilfsbedürftigkeit der alternden Eltern adäquat entsprechen zu können.

Da die Klärung dieser Kausalkette nicht Gegenstand der aktuellen Untersuchung war, kann an diesem Punkt darüber nur spekuliert werden. In zukünftigen Studien sollte diesem Aspekt jedoch sicherlich größere Aufmerksamkeit zukommen.

Darüber hinaus ist den Ergebnissen der aktuellen Studie zufolge eine mit Hilfe der FAB gemessene erhöhte Sorge der betreuenden Söhne und Töchter um den abnehmenden gesundheitlichen Zustand und der damit verbundenen zu erwartenden Hilfsbedürftigkeit der Eltern (filiale Angst B) eng mit einer erhöhten subjektiven Belastung durch die Betreuung assoziiert. Der Zusammenhang erscheint vordergründig nahe liegend: Die erwartete zukünftige Verschlechterung des körperlichen und geistigen Zustandes des erkrankten Elternteils wird als belastend erlebt. Weiterhin und demgegenüber kann anhand der vorliegenden Studie auch gezeigt werden, dass eine schlechtere körperliche Funktionsfähigkeit der betreuenden Kinder sowie eine höhere Prävalenz depressiver Symptome, welche als physische und psychische Zeichen eines erhöhten Belastungserlebens gelten, mit einer größeren Angst der Angehörigen vor dem erwarteten zukünftigen Nachlassen des körperlichen und geistigen Zustandes der Eltern einhergehen.

Inwiefern die filiale Angst B der betreuenden erwachsenen Kinder also tatsächlich Ursache oder Wirkung ihres insgesamt erhöhten Belastungserlebens ist, kann daher ebenfalls nicht abschließend geklärt werden. 
Anhand der Untersuchungsergebnisse lässt sich neben dem Zusammenhang zwischen filialer Angst und Pflegebelastung auch ein gewisser Einfluss der filialen Reife auf das Belastungserleben der betreuenden Kinder der Parkinsonpatienten belegen. Die sieben verschiedenen Faktoren der filialen Reife zeigen dabei unterschiedlich starke Assoziationen mit der empfundenen allgemeinen Belastung. So zeigen sich diesbezüglich insbesondere hohe Korrelationen mit den Reife-Faktoren „filiales Verpflichtungsgefühl“, „filiale Autonomie“ und „familiäre Solidarität“. Im Einklang mit den Ergebnissen verschiedener anderer Studien führen demnach ein hohes filiales Verpflichtungsgefühl sowie eine als gering empfundene familiäre Solidarität zu einer Verstärkung der Belastung (Linkersdörfer 2006; Lowenstein 1999; Schmidt 2009; Schütze und Wagner 1991). Eine hohe filiale Autonomie scheint sich hingegen protektiv auf das Belastungserleben der betreuenden Angehörigen auszuwirken.

Wie bereits erwähnt, lässt sich auch anhand der Ergebnisse der aktuellen Studie der Zusammenhang zwischen einem hohen filialen Verpflichtungsgefühl der Studienteilnehmer und einer subjektiv als stark empfundenen Belastung durch die Betreuung der erkrankten Eltern eindeutig belegen. Zudem scheint sich das filiale Verpflichtungsgefühl negativ auf die „körperliche Funktionsfähigkeit“ der betreuenden Kinder auszuwirken. Dieser Fund wird durch Studienergebnisse verschiedener anderer Autoren bestätigt (vgl. Linkersdörfer 2006; Schmidt 2009). Auch Raveis und Kollegen kamen in ihrer Untersuchung zu dem Schluss, dass ein höheres filiales Verpflichtungsgefühl in einem direkten Zusammenhang mit einer erhöhten Pflegebelastung der erwachsenen Kinder steht. Umgekehrt wirke sich eine positive Einstellung zur Pflege auch positiv auf die empfundene Belastung aus (Raveis et al. 1998). Sicherlich interessant ist hierbei die Frage, inwiefern ein vergleichsweise hohes filiales Verpflichtungsgefühl auf die eigene intrinsische Motivation des betroffenen Kindes zurückzuführen ist oder - wie in diesem Kapitel bereits angedeutet - dem erwachsenen Kind doch eher durch in unserer Gesellschaft bestehende Normen und Moralvorstellung sowie die elterliche Erziehung „von außen“ auferlegt ist. In diesem Fall wäre die Entscheidung zur Pflegeübernahme der Eltern mit einem stärkeren Gefühl des Zwangs und der Unfreiwilligkeit verbunden. Die erbrachten Leistungen würden so als belastender und einschränkender empfunden. Ein vorrangig aus äußeren Einflüssen resultierendes erhöhtes filiales Verpflichtungsgefühl wäre auch als Anzeichen einer eher gering ausgeprägten filialen Reife des betreuenden Kindes zu werten, da laut Bruder ein hilfeleistendes Verhalten nur dann als „filial reif“ gelten kann, wenn es freiwillig und nicht durch von außen aufgezwungene Schuldgefühle erfolgt (Bruder 1988).

Die hohe Bedeutung der familiären Solidarität für das Belastungserleben der betreuenden Angehörigen spiegelt sich insbesondere durch die positiven Korrelationen mit den sozialen Domänen der Gesundheitsbezogenen Lebensqualität wie der „sozialen Funktionsfähigkeit“, der „emotionalen Rollenfunktion“ und des „,psychischen Wohlergehens“ der betreuenden Kinder wider. Auch kommt es 
der aktuellen Untersuchung zufolge bei den Studienteilnehmern, die sich in ihrer betreuenden Position durch ihre Familienmitglieder gut unterstützt fühlen, weniger häufig zur Ausbildung depressiver Symptome. Eine als positiv erlebte familiäre Solidarität scheint demnach zu einer Verringerung des allgemeinen Belastungserlebens der betreuenden Angehörigen zu führen (vgl. auch Linkersdörfer 2006; Schmidt 2009; Stiens 2000).

Es ist anzunehmen, dass eine größere familiäre Solidarität mit weniger innerfamiliären Konflikten und Streitigkeiten verbunden ist, welche sich als zusätzliche Stressoren negativ auf die bestehende Pflegebeziehung und somit auch indirekt negativ auf die allgemeine Belastung des erwachsenen Kindes durch die Betreuung des erkrankten Elternteils auswirken. Darüber hinaus ist eine als positiv erlebte familiäre Solidarität sicherlich auch mit einer angemessenen Unterstützung - und sei sie nur emotionaler Art - der hauptsächlich pflegenden bzw. betreuenden Person verbunden. Laut England und Tripp-Reimer ist die mangelnde Unterstützung von Seiten der Geschwister einer der Hauptfaktoren für ein erhöhtes Belastungserleben der betreuenden Kinder (England und Tripp-Reimer 2003). Eine gerechtere Verteilung der Verantwortung durch eine vermehrte Einbeziehung der nicht oder weniger pflegenden Geschwister führt in „solidarischen“ Familien zu einer als stärker empfundenen Unterstützung und somit zu einer als geringer empfundenen Belastung des hauptsächlich betreuenden Kindes (Chappell und Reid 2002). Auch Marcoen weist im Rahmen seiner Ausführungen zur filialen Reife auf den hohen Stellenwert der Familiensolidarität in der Betreuung hilfsbedürftiger Angehöriger hin. Eine gute familiäre Solidarität führe ihm zufolge nicht nur zu einer gerechteren Verteilung der Pflegeverantwortung, sondern gebe auch die Möglichkeit, die eigenen Fähigkeiten und Grenzen in der Betreuungssituation besser einschätzen und akzeptieren zu können. Eine realistische Einschätzung dieser Fähigkeiten stehe in direktem Zusammenhang mit der subjektiven Belastung der erwachsenen Kinder durch die Betreuung der alternden Eltern (Marcoen 1995).

Die filiale Autonomie der Studienteilnehmer steht zwar nicht in einem direkten Zusammenhang mit dem Fragebogen zur Belastung durch die Betreuung, es zeigen sich jedoch eindeutig positive Korrelationen mit verschiedenen Bereichen der Gesundheitsbezogenen Lebensqualität, was ebenfalls als Maß für die subjektive Belastung der betreuenden Angehörigen dient. Die betreuenden Söhne und Töchter mit einer verhältnismäßig hohen filialen Autonomie geben demnach im Vergleich eine bessere „körperliche Funktionsfähigkeit“ und „Rollenfunktion“ sowie eine als besser bewertete „allgemeine Gesundheit“" an. Bestätigung findet dieser Fund in der diesbezüglichen Fachliteratur: Die filiale Autonomie wird übereinstimmend als zentraler Faktor filialer Reife in der Betreuung hilfsbedürftiger Eltern beschrieben. Eine angemessene filiale Autonomie ermögliche dem betreuenden Kind einen leichteren und besseren Umgang mit der Pflegesituation und habe so einen positiven Einfluss auf dessen allgemeines Belastungserleben (Adler et al. 1996; Blenkner 1965; Haenselt et al. 2004). Nach Bruder (1988) kann nur ein emotional unabhängiges erwachsenes Kind, den schrittweisen 
Verlust eines Elternteils in seiner aktiv pflegenden Rolle erleben. Andernfalls komme es infolge des Wiederauflebens alter Konflikte zu Spannungen innerhalb der Pflegebeziehung und damit zu einer erhöhten Belastung der pflegenden Person (Bruder 1988).

Interessanterweise zeigen sich diesbezüglich in den bereits wiederholt zitierten Vorstudien von Stiens (2000), Linkersdörfer (2006) und Schmidt (2009) gegensätzliche Ergebnisse: In allen drei Untersuchungen konnten keine nennenswerten Zusammenhänge zwischen dem Grad der filialen Autonomie der betreuenden Kinder und dem Ausmaß ihrer Belastung durch die Pflege belegt werden. Hinweise für eine mögliche Ursache dieser Diskrepanz könnten bei der Filiale-Reife-Skala selbst sowie den unterschiedlichen Studienpopulationen zu finden sein. Die Filiale-Reife-Skala beinhaltet zwei Kategorien von Aussagen bezüglich der filialen Autonomie. Zum einen wird erfragt, inwieweit die erwachsenen Kinder unabhängig vom Rat, Beistand und Einfluss ihrer Eltern leben (emotionale Autonomie), zum anderen wie sehr sich die Kinder ihrerseits in das Leben der Eltern einmischen (operative Autonomie).

In den drei besagten Vorstudien standen jeweils erwachsene Kinder demenzkranker Eltern im Mittelpunkt der Untersuchungen. Wie schon in Kapitel 4.1.1 beschrieben, sind diese demenzkranken Eltern nicht nur wesentlich älter sondern auch wesentlich hilfs- und pflegebedürftiger als die an dem idiopathischen Parkinson-Syndrom erkrankten Eltern der aktuellen Studienteilnehmer. Es muss also überlegt werden, ob eine vergleichsweise höhere Hilfsbedürftigkeit der Eltern den pflegenden Kindern der Vorstudien eventuell weniger Abgrenzung ermöglicht und somit auch eine stärkere Einmischung in die elterlichen Belange fordert als das bei den betreuenden Söhnen und Töchtern in der vorliegenden Untersuchung der Fall ist. Möglicherweise könnte so eine höhere Hilfsbedürftigkeit die eigentlich größere emotionale Autonomie ,ausgleichen“.

Die filiale Liebe und die elterliche Besorgnis scheinen den Ergebnissen dieser Untersuchung zufolge keine besondere Bedeutung für das Belastungserleben der betreuenden Angehörigen zu haben. Es finden sich hier lediglich positive Korrelationen mit dem „psychischen Wohlbefinden“. Eine hohe den Eltern gegenüber empfundene Liebe sowie eine hohe elterliche Besorgnis sind demnach mit einem guten psychischen Wohlbefinden der betreuenden Söhne und Töchter verbunden. Auch scheint eine als gut empfundene elterliche Einsicht tendenziell mit weniger psychischen Problemen der betreuenden Kinder, insbesondere der Töchter, verbunden zu sein.

Die filiale Liebe ist laut Marcoen eine der zentralen Dimension filialer Reife und zugleich der stärkste Motivator für die Bereitschaft zur Übernahme der elterlichen Pflege (Marcoen 1993). Denkbar wäre hierbei, dass eine schlechtere Beziehung zu den Eltern mit einer vergleichsweise geringen filialen Liebe zu einer geringeren Freiwilligkeit der Pflegeleistungen führen könnte. Die daraus resultierende unangenehmere Atmosphäre könnte so wiederum zu einer Verstärkung des Belastungserlebens der betreuenden Kinder führen. 
Die elterliche Besorgnis oder auch elterliche Einsicht hingegen signalisiert den betreuenden Kindern, dass sich die Eltern als pflegeempfangene Personen der Belastung der Kinder bewusst sind, diese anerkennen und sich dementsprechend bemühen, die Kinder ihrerseits durch entgegenkommendes Verhalten wie beispielsweise das Einplanen und Gewähren von Ruhepausen zu entlasten. Eine als gut empfundene elterliche Einsicht könnte sich so als Schutzfaktor mindernd auf das kindliche/ filiale Belastungserleben auswirken.

Die filiale Liebe und die elterliche Besorgnis charakterisieren folglich das Verhältnis zwischen alternden Eltern und erwachsenen Kindern als eine auf Gegenseitigkeit beruhende Beziehung. Schon für Marcoen beinhaltete eine gute intergenerationelle Beziehung die „Bi-Direktionalität“ und die Reziprozität der Beziehung (Marcoen 1995 und 2005). Die Art und Qualität der Beziehung zu den hilfsbedürftigen Eltern bestimmt somit auch wesentlich die Belastungsverarbeitung der betreuenden Kinder mit (Adler et al. 1996; Linkersdörfer 2006; Wilz et al. 1998). Im Prinzip würde man daher auch sicherlich einen größeren Einfluss dieser Faktoren der filialen Reife auf das Belastungserleben der Studienteilnehmer erwarten als es die Ergebnisse der aktuellen Untersuchung vermuten lassen. Ein Erklärungsversuch hierfür könnte lauten, dass die an dem idiopathischen Parkinsonsyndrom erkrankten Eltern der aktuellen Studienteilnehmer zwar körperlich schon teilweise stark beeinträchtigt sind, die Einschränkungen ihrer kognitiven Fähigkeiten durch die Erkrankung jedoch noch relativ gering sind. Das Ausmaß ihrer elterlichen Besorgnis und Einsicht könnte demnach noch als weitgehend „normal“ und durch die Erkrankung unbeeinträchtigt angesehen werden. Weiterhin kann vermutet werden, dass sich die positiven Einflüsse einer hohen filialen Liebe mit den negativen Einflüssen wie beispielsweise einer stärkeren Anteilnahme am elterlichen Schicksal und größeren Schwierigkeiten, den krankheitsbedingten Abbau des geliebten Elternteils zu ertragen, ausgleichen. Der Fund, dass sich die betreuenden erwachsenen Söhne und Töchter dieser Untersuchung weder in ihrer filialen Liebe noch in der empfundenen elterlichen Besorgnis signifikant von ihren Kontrollpersonen unterscheiden, stützt diese These. Der im Vergleich zu anderen Studien geringe Einfluss der Reife-Faktoren „filiale Liebe“ und „elterliche Besorgnis“ auf das Belastungserleben der betreuenden Angehörigen könnte auf diese Weise erklärt werden (vgl. Linkersdörfer 2006; Schmidt 2009 und Stiens 2000).

Auch die filiale Hilfe und die filiale Hilfsbereitschaft scheinen keinen nennenswerten Einfluss auf das Belastungserleben der Studienteilnehmer zu haben. Es zeigen sich hier lediglich Zusammenhänge zwischen einer hohen filialen Hilfsbereitschaft und einem guten ,psychischen Wohlbefinden“ der betreuenden Angehörigen sowie zwischen einem hohen Maß an filialer Hilfe und einer schlechteren „körperlichen Funktionsfähigkeit“. Letztere Verbindung ist bei den betreuenden Töchtern mit $\mathrm{p}<0,01$ besonders deutlich. Je mehr Hilfe geleistet wird, desto stärker wird das eigene Leben eingeschränkt. Entsprechend weniger Zeit verbleibt den betreuenden Kindern zur körperlichen Regeneration. Die 
körperliche Belastungsgrenze ist so schneller erreicht, was in einer schlechteren Einschätzung der körperlichen Funktionsfähigkeit resultiert.

An dieser Stelle sei noch einmal das Augenmerk auf die insgesamt tendenziell höhere Pflegebelastung der Frauen in Verbindung mit der filialen Angst bzw. mit den Faktoren der filialen Reife gelegt.

Wie bereits von Schütze und Wagner 1991 beschrieben, sind es auch heute noch in erster Linie die Töchter, die sich aufgrund des noch immer weit verbreiteten traditionellen Rollenverständnisses eher in der Pflicht sehen, die Betreuung und Pflege ihrer hilfsbedürftigen Eltern zu übernehmen (vgl. auch Perrig-Chiello und Sturzenegger 2001). Pflegende Söhne hingegen werden - ähnlich wie allein erziehende Väter - von ihrer Umgebung immer noch eher als „positive Enttäuschung“ wahrgenommen (Schütze und Wagner 1991). Vermutlich verspüren sie so diesbezüglich auch einen geringeren Druck und die Beeinflussung ihres filialen Verpflichtungsgefühls „von außen“ ist dementsprechend auch geringer. Im Falle der Pflegeübernahme sei es daher nicht verwunderlich, wenn sich für betreuende Söhne ein Zuwachs an Selbstbewusstsein und somit auch ein positiver Effekt auf deren subjektive Belastung ergebe (Schütze und Wagner 1991).

Ein weiterer Grund für das geringere Belastungserleben betreuender Söhne ist die Tatsache, dass Söhnen insbesondere durch ihre Schwestern mehr Hilfe gewährt wird als hauptsächlich betreuenden Töchtern (Brody et al. 1989). Auch erfahren betreuende Söhne in der Regel häufiger und mehr Unterstützung durch die eigene Partnerin (Faison et al. 1999). Die familiäre Solidarität hat hier also durch eine bessere Verteilung der Verantwortung einen positiven Effekt auf das allgemeine Belastungserleben.

Darüber hinaus könnten die „typisch weibliche“ größere Mühelosigkeit, über belastende Faktoren zu sprechen, sowie die „typisch männliche“ Tendenz, emotionale Themen eher zu meiden, einen nicht unwesentlichen Einfluss auf die geschlechtsspezifisch unterschiedlich starken Korrelationen zwischen der filialen Angst bzw. Reife und der Belastung der betreuenden Söhne und Töchter haben.

\subsubsection{Zusammenfassung Diskussion Hypothese 1}

Letztendlich kann festgehalten werden, dass anhand der vorliegenden Untersuchungsergebnisse die erste Hypothese bestätigt werden kann, nach der ein enger Zusammenhang zwischen der filialen Angst bzw. Reife erwachsener Kinder alternder Eltern und deren subjektivem Belastungserleben besteht: Betreuende Kinder von hilfsbedürftigen Eltern, die an dem idiopathischen Parkinsonsyndrom erkrankt sind, werden von einer verhältnismäßig hohen filialen Angst sowie einem geringen Ausmaß an filialer Reife in der Betreuungssituation eingeschränkt, was sich in einer erhöhten Belastung der erwachsenen Kinder widerspiegelt. Die Sorge der betreuenden Kinder vor dem erwarteten zukünftigen Nachlassen des körperlichen und geistigen Zustands des erkrankten Elternteils (filiale Angst B) scheint dabei einen stärkeren Einfluss auf die empfundene Belastung zu haben als ihre Angst bezüglich der eigenen Fähigkeiten, der erwarteten Rolle als pflegende Personen gerecht zu werden (filiale Angst A). 
Inwieweit sich diese filialen Ängste A und B gegenseitig beeinflussen, wäre sicherlich eine interessante Fragestellung für zukünftige Untersuchungen.

Bezüglich des Zusammenhangs zwischen den sieben Faktoren der filialen Reife und dem Belastungserleben scheinen insbesondere die Faktoren „filiales Verpflichtungsgefühl“, „filiale Autonomie“ und „familiäre Solidarität" eine zentrale Rolle zu spielen. So wirken sich ein starkes filiales Verpflichtungsgefühl eindeutig negativ, eine große emotionale Autonomie der erwachsenen Kinder sowie eine als positiv empfundene Familiensolidarität hingegen protektiv auf die Belastung der betreuenden Angehörigen aus. Ein in diesem Zusammenhang interessantes Ergebnis ist der Fund, dass ein ungünstiges $\mathrm{Ma} ß$ an filialem Verpflichtungsgefühl und filialer Autonomie vorzugsweise mit körperlichen Symptomen der Studienteilnehmer, eine schlechte familiäre Solidarität hingegen vermehrt mit Einschränkungen im psycho-sozialen Bereich einhergehen.

\subsection{Diskussion Hypothese 2}

Die zweite Hypothese befasste sich mit der Frage, mit welcher der beiden Bögen, Filial Anxiety Scale nach Cicirelli (FAS) oder Louvain Filial Maturity Scale A nach Marcoen (LFMS-A), das Belastungserleben der Studienteilnehmer besser korreliert.

Die Filiale Angst B der betreuenden Kinder korreliert hochsignifikant positiv mit dem Fragebogen zur Belastung durch die Betreuung. Diese statistisch signifikante Verbindung zeigt sich zudem isoliert in der Untergruppe der betreuenden Töchter. Die positive Korrelation zwischen der filialen Angst A und dem Belastungsbogen verfehlt mit $\mathrm{p}<0,0505$ die geforderte statistische Signifikanz nur denkbar knapp. Bei den Faktoren der filialen Reife Skala findet sich für die Gesamtstichprobe lediglich eine signifikant positive Korrelation zwischen dem „filialen Verpflichtungsgefühl“ und dem Fragebogen zur Belastung durch die Betreuung. In der Untergruppe der betreuenden Töchter spielt neben dem „filialen Verpflichtungsgefühl“ zudem noch die „familiäre Solidarität“ eine signifikante Rolle für das subjektive Belastungserleben.

Die Untersuchungsergebnisse unterstützen folglich Hypothese 2, nach der die mittels der FAS gemessene filiale Angst der betreuenden erwachsenen Kinder von Parkinsonpatienten in einem engeren Zusammenhang mit ihrer subjektiv empfundenen Belastung steht als ihre anhand der LFMSA erhobene filiale Reife. Die Filial Anxiety Scale scheint demnach besser geeignet zu sein, anhand des Ausmaßes der filialen Angst die Situation der betreuenden erwachsenen Kinder innerhalb der Pflege zu erfassen als der Filiale-Reife-Bogen. Die filiale Angst könnte so als Prädiktor für das Belastungserleben betreuender Angehöriger dienen.

Hieraus ergeben sich Konsequenzen für den klinischen Einsatz der Fragebögen und die Beratung pflegender Angehöriger in der Praxis. Wie schon in den Kapiteln 2.4.11 und 2.4.12 dargestellt, unterscheiden sich die Filial Anxiety Scale und die Louvain Filial Maturity Scale A nicht nur in ihren 
Zielgrößen sondern auch in ihrem Aufbau. Während die Filiale-Angst-Skala aus dreizehn Fragen besteht, von denen die Punktwerte der ersten sieben Fragen zur Filialen Angst A und die der Fragen 8 bis 13 zur Filialen Angst B addiert werden, beinhaltet der Filiale-Reife-Bogen 81 Aussagen zur elterlichen Beziehung, deren Beantwortung im Vergleich deutlich mehr Zeit beansprucht. Vor der eigentlichen Auswertung des Bogens müssen diese Aussagen zudem erst den sieben Faktoren der filialen Reife zugeordnet werden, was ebenfalls relativ viel Zeit im Auswertungsprozess beansprucht. Cicirellis Filial Anxiety Scale ist somit nicht nur aus Sicht der pflegenden Angehörigen, denen die Skala im Rahmen der Angehörigenberatung vorgelegt werden könnte, sondern auch für die Berater zeitsparender und damit besser einsetzbar als die Louvain Filial Maturity Scale A nach Marcoen.

\subsubsection{Zusammenfassung Diskussion Hypothese 2}

Zusammenfassend bleibt daher festzustellen, dass die zweite Hypothese dieser Untersuchung bestätigt werden kann. Die Filiale-Angst-Skala spiegelt im Vergleich mit dem Filiale-Reife-Bogen die subjektive Belastung der betreuenden erwachsenen Kinder, welche sie durch die Betreuung ihres an dem idiopathischen Parkinsonsyndrom erkrankten Elternteils erfahren, besser wieder. Zudem zeichnet sie sich durch ihre größere Anwendungsfreundlichkeit in der Praxis aus.

\subsection{Diskussion Hypothese 3}

Der erste Teil der dritten Hypothese lautete, dass sich Schlafstörungen der betreuenden erwachsenen Kinder negativ auf ihr Belastungserleben und ihre gesundheitsbezogene Lebensqualität auswirken. Anhand der vorliegenden Ergebnisse kann dies lediglich teilweise bestätigt werden. So konnten weder Zusammenhänge zwischen dem Ausmaß der Tagesschläfrigkeit (Epworth Sleepiness Scale) und der subjektiven Belastung durch die Betreuung noch zwischen der allgemeinen Schlafqualität (Gesamtwert des Pittsburgh Sleep Quality Index) und dem Belastungsbogen festgestellt werden. Lediglich die „subjektive Schlafqualität“ (Unterscore 1 der PSQI) der betreuenden Söhne und Töchter steht in einem signifikanten Zusammenhang mit dem Ausmaß ihrer durch die Pflegesituation empfundenen Belastung. Ergebnissen von Cifu und Mitarbeitern zufolge besteht hingegen eine eindeutige Verbindung zwischen der Pflegebelastung und der Schlafdauer betreuender Angehöriger von Parkinsonpatienten (Cifu et al. 2006). Auch Creese et al. sowie Phillips et al. bestätigen einen Zusammenhang zwischen einer reduzierten allgemeinen Schlafqualität betreuender Angehöriger und einer erhöhten Belastung durch die Pflegerolle (Creese et al. 2008; Phillips et al. 2009), während Wilcox und King jene Assoziation nicht nachweisen konnten (Wilcox und King 1999). Laut einer Studie von Happe und Berger ist neben dem Belastungserleben pflegender Ehepartner von Parkinsonpatienten auch das Ausmaß ihrer psychosomatischen Beschwerden und die Frequenz der 
geleisteten Pflege mit einem schlechten Nachtschlaf der Betroffenen verbunden (Happe und Berger 2002).

Eindeutige Zusammenhänge lassen sich den aktuellen Ergebnissen zufolge hingegen zwischen der allgemeinen Schlafqualität und den körperlichen und mentalen Komponenten der gesundheitsbezogenen Lebensqualität der betreuenden Kinder belegen. So ist eine relativ schlechte allgemeine Schlafqualität hochsignifikant mit einer als niedrig empfundenen „körperlichen Funktionsfähigkeit“ und „Rollenfunktion“ der Studienteilnehmer sowie einer eingeschränkten ,allgemeinen Gesundheit“ und „Vitalität“ verbunden. Auch das „psychische Wohlbefinden“ der betreuenden Kinder wird durch eine schlechte allgemeine Schlafqualität negativ beeinflusst. Diese deutlichen Zusammenhänge lassen sich gut in bisherige Forschungsergebnissen einordnen. So berichten Cifu et al. beispielsweise, dass sich eine Fragmentierung des Nachtschlafes negativ auf die physische und psychische Gesundheit betreuender Angehöriger auswirkt. Ein direkter Zusammenhang zwischen dem allgemeinen Gesundheitsstatus und dem Belastungserleben besteht hingegen nicht (Cifu et al. 2006). Darüber hinaus sehen Creese und Kollegen aufgrund ihrer Studienergebnisse eine erhöhte Frequenz nächtlicher Schlafstörungen mit einer Verschlechterung der mentalen Gesundheit der Betroffenen assoziiert. In der körperlichen Gesundheit spiegeln sich ihnen zufolge eine eingeschränkte Schlafqualität allerdings nicht wieder (Creese et al. 2008). Cupidi et al. wiesen ebenfalls einen direkten Zusammenhang zwischen einer erhöhten Prävalenz an Schlafstörungen und einer verminderten Lebensqualität bei betreuenden Angehörigen von Alzheimer- und Parkinsonpatienten nach - mit Ausnahme des Einflusses auf die körperlichen Domänen der Lebensqualität (Cupidi et al. 2012). Willette-Murphy und Mitarbeiter berichten zudem sowohl von direkten als auch indirekten Auswirkungen der Belastung durch die Betreuung eines pflegebedürftigen Familienmitglieds auf die mentale Gesundheit der betreuenden Personen. Ihnen zufolge sei eine reduzierte Schlafeffizienz auch als durchaus hinweisend für eine Beeinträchtigung der Psyche pflegender Ehefrauen anzusehen (Willette-Murphy et al. 2006). Phillips et al. stellten zudem fest, dass nicht nur Pflegende mit Schlafstörungen vermehrt depressive Symptome angaben sondern auch, dass Schlafstörungen als vermittelnder Faktor (,mediator") für das Verhältnis zwischen einer erhöhten Pflegebelastung und dem Auftreten depressiver Symptome bei den Pflegenden anzusehen ist (Phillips et al. 2009, vgl. auch Cupidi et al. 2012).

Erwähnenswert ist, dass die Schlafqualität bei den betreuenden Söhnen einen sehr viel geringeren Einfluss auf ihre gesundheitsbezogene Lebensqualität hat als bei den Töchtern. Während bei letzteren eine verminderte allgemeine Schlafqualität signifikant mit einer als schlechter empfundenen „körperlichen Funktionsfähigkeit“ und „Rollenfunktion“ sowie einer verminderten „Vitalität“ und einem reduzierten „psychischen Wohlbefinden“ zusammenhängt, besteht bei den betreuenden Söhnen lediglich eine Assoziation zwischen einer verminderten Schlafqualität und einer Einschränkung ihrer 
„allgemeinen Gesundheit“. Da - wie bereits in Kapitel 4.2.7 erwähnt - Männer generell auf psychische Belastungen weitaus seltener mit Einschränkungen ihrer Schlafqualität reagieren als Frauen, ist dieser Befund jedoch nicht weiter verwunderlich. Zudem könnte die ohnehin geringe Anzahl von betreuenden Söhnen mit einer eingeschränkten Schlafqualität an der Gesamtstichprobe zu dem geringen Ausmaß signifikanter Korrelationen beigetragen haben.

Zusammenhänge zwischen dem Ausmaß der Tagesschläfrigkeit und dem durch die Betreuung subjektiv empfundenen Belastungserleben bzw. der gesundheitsbezogenen Lebensqualität der erwachsenen Kinder konnten in der aktuellen Untersuchung nicht festgestellt werden. Bei den betreuenden Söhnen wurde zwar eine signifikante positive Korrelation zwischen der Tagesschläfrigkeit und ihrer „körperlichen Rollenfunktion“ gefunden. In der Folge hieße dies jedoch, dass betreuende Söhne, die unter einer hohen Tagesschläfrigkeit leiden, ihre „körperliche Rollenfunktion“ als besonders gut empfinden. Da dies allen bisherigen Ergebnissen der vorliegenden Untersuchung entgegensteht, soll diese Korrelation als Artefakt gewertet werden.

Dass sich das durch die Epworth Sleepiness Scale (ESS) gemessene Ausmaß der Tagesschläfrigkeit der betreuenden erwachsenen Kinder insgesamt weniger stark auf deren Belastungserleben und deren gesundheitsbezogene Lebensqualität auswirkt als die mit Hilfe des Pittsburgh Sleep Quality Index (PSQI) ermittelte allgemeine Schlafqualität der Studienteilnehmer ist nicht weiter verwunderlich. Schon Buysse und Mitarbeiter, die in einer Studie die ESS und den PSQI auf ihre jeweilige Assoziation mit subjektiven und objektiven Schlafcharakteristika untersuchten, bestätigten, dass der PSQI in einem wesentlich engeren Zusammenhang mit psychologischen Symptomen und Schlaftagebucheinträgen der teilnehmenden Probanden steht als die ESS (Buysse et al. 2008).

Im zweiten Teil der dritten Hypothese wurde der gegenseitige Einfluss von Schlafstörungen und der gesundheitsbezogenen Lebensqualität der Parkinsonpatienten untersucht. Auch hier bestätigt sich die Hypothese, dass eine erhöhte Tagesschläfrigkeit und eine reduzierte Schlafqualität mit einer Beeinträchtigung der gesundheitsbezogenen Lebensqualität der Betroffenen einhergehen. Eine hohe Tagesschläfrigkeit scheint demnach insbesondere die „körperliche Funktionsfähigkeit“ der Probanden einzuschränken. Zudem werden die körperliche und emotionale Rollenfunktion sowie die ,allgemeine Gesundheit" der männlichen Patienten durch ein hohes Maß an Tagesschläfrigkeit negativ beeinflusst. Darüber hinaus bestehen mit $\mathrm{p}<0,01$ deutliche Zusammenhänge zwischen einer schlechten allgemeinen Schlafqualität und einer niedrigen „Vitalität“ sowie einem eingeschränkten „psychische Wohlbefinden“ der Parkinsonpatienten. Einschränkungen in den SF-36-Bereichen „körperliche“ und „soziale Funktionsfähigkeit“ und „allgemeine Gesundheit“ sind ebenfalls mit einer schlechten allgemeinen Schlafqualität verbunden. Zudem scheinen sich besonders die körperlichen Schmerzen der Parkinsonpatienten in einer schlechten allgemeinen Schlafqualität zu reflektieren. 
Die Ergebnisse dieser Untersuchung stehen somit insgesamt im Einklang mit zahlreichen bisherigen Forschungsergebnissen (Happe und Berger 2003; Havlikova et al. 2011; Karlsen et al. 1999 und 2000; Scaravilli et al. 2003; Simuni und Sethi 2008; Visser et al. 2008). Laut Happe und Berger hat eine hohe Frequenz an Schlafstörungen einen eindeutig negativen Einfluss auf die gesundheitsbezogene Lebensqualität von Parkinsonpatienten (Happe und Berger 2003). Ebenfalls signifikant ist der Zusammenhang zwischen einer eingeschränkten Schlafqualität und der Prävalenz depressiver Symptome (vgl. auch Svensson et al. 2012). Aufgrund des longitudinalen/prospektiven Charakters ihrer Studie konnten Happe und Berger zudem eine Aussage über den Einfluss von Veränderungen in der Schlafqualität auf die Lebensqualität der Patienten über einen Zeitraum von elf Monaten machen. Während demnach Patienten, die zum Zeitpunkt der zweiten Datenerhebung ein häufigeres Vorkommen von Schlafstörungen als beim ersten Termin angaben, eine Verschlechterung in allen drei erhobenen Domänen (körperliche Funktionsfähigkeit, allgemeine Gesundheit, psychisches Wohlbefinden) ihrer Lebensqualität feststellten, berichteten Patienten, die am Zeitpunkt 2 Schlafstörungen von vergleichsweise niedrigerer oder unveränderter Intensität angaben, von einer Verbesserung ihrer gesundheitsbezogenen Lebensqualität in wenigstens einem der genannten Bereiche (Happe und Berger 2003). Studienergebnissen von Karlsen und Kollegen zufolge wirkt sich neben selbst berichteter Schlaflosigkeit und depressiven Symptomen auch der Grad der körperlichen Abhängigkeit der Parkinsonpatienten negativ auf ihre subjektive Lebensqualität aus (Karlsen et al. 1999). Darüber hinaus berichten Visser und Mitarbeiter von einer Beeinträchtigung der gesundheitsbezogenen Lebensqualität von Parkinsonpatienten aufgrund von starker Tagesschläfrigkeit (Visser et al. 2008). Auch Havlikova et al. (2011) stellten anhand ihrer Forschungsergebnisse signifikante Zusammenhänge zwischen einer reduzierten Lebensqualität von Parkinsonpatienten und einer vermehrten Tagesschläfrigkeit sowie einer insgesamt eingeschränkten Schlafqualität fest. Allgemeine Schlafstörungen seien zudem eindeutig mit einer erhöhten Prävalenz von Depressionen und Angst verbunden (Havlikova et al. 2011). Derlei signifikante Zusammenhänge zeigen sich - wie bereits beschrieben - auch bei dem Patientenkollektiv der vorliegenden Untersuchung und bestehen hier vorrangig mit den körperlichen Aspekten der gesundheitsbezogenen Lebensqualität.

Als weiteren Aspekt untersuchten Havlikova und Kollegen den Einfluss der durch die Parkinsonerkrankung hervorgerufenen allgemeinen Erschöpfung der Patienten („,fatigue“; vgl. Kapitel 1.1.7) auf ihre gesundheitsbezogene Lebensqualität. Die mentale Erschöpfung beeinträchtigt demnach besonders die psychologischen Bereiche der Lebensqualität wie emotionales Wohlbefinden, soziale Unterstützung und Kommunikation, während sich die körperliche Ermüdung der Patienten negativ auf ihre Mobilität, die Aktivitäten des täglichen Lebens sowie das körperliche Wohlbefinden der Betroffenen auswirkt (Havlikova et al. 2008). Auch Herlofson und Larsen berichten in ihrer Studie über oben genannten Zusammenhang. Einschränkungen in der gesundheitsbezogenen Lebensqualität durch die krankheitsbedingte allgemeine Erschöpfung der Parkinsonpatienten fanden sich ihnen 
zufolge besonders in den SF-36-Bereichen „körperliche Funktionsfähigkeit“, „körperliche“ und „soziale Rollenfunktion“ sowie „Vitalität“ (Herlofson und Larsen 2003).

Interessant ist, dass bei den männlichen Patienten dieser Untersuchung häufiger signifikante Korrelationen zwischen der Tagesschläfrigkeit bzw. Schlafqualität und den verschiedenen Bereichen ihrer gesundheitsbezogenen Lebensqualität festgestellt werden konnten als bei den weiblichen Probandinnen. Da Frauen im Allgemeinen sensibler auf Einschränkungen ihres körperlichen und seelischen Wohlbefindens mit Schlafstörungen reagieren als Männer, steht dieser Fund im Gegensatz zu bisherigen Forschungsergebnissen (vgl. Kapitel 4.2.7). Allerdings gehen Schlafstörungen bei Frauen auch in geringerem Maße mit einer vermehrten Tagesschläfrigkeit einher als bei Männern, die ihrerseits häufiger ein Schlafapnoe-Syndrom haben. So steht dieses Ergebnis letztendlich nicht unmittelbar im Widerspruch zu den übrigen Ergebnissen.

Schlüssig erscheint hingegen die Tatsache, dass die Parkinson's Disease Sleep Scale (PDSS) besser mit der gesundheitsbezogenen Lebensqualität der Parkinsonpatienten korreliert als der Pittsburgh Sleep Quality Index (PSQI). Bei der PDSS handelt es sich um einen Parkinson-spezifischen Schlaffragebogen, der eigens auf die im Rahmen einer Parkinsonerkrankungen auftretenden Schlafstörungen abgestimmt wurde. Daher ist davon auszugehen, dass die PDSS Schlafstörungen bei Parkinsonpatienten besser erfasst als der PSQI, der im Hinblick auf die allgemeine Schlafqualität bei jeglichen Studienpopulationen und Altersklassen eingesetzt werden kann, und so auch eine größere Anzahl signifikanter Korrelationen mit der gesundheitsbezogenen Lebensqualität aufweist.

\subsubsection{Zusammenfassung Diskussion Hypothese 3}

Zusammenfassend kann festgehalten werden, dass auch die dritte Hypothese durch die Ergebnisse der vorliegenden Untersuchung größtenteils bestätigt werden kann. Schlafstörungen führen demnach sowohl bei den betreuenden erwachsenen Kindern als auch bei deren an dem idiopathischen Parkinsonsyndrom erkrankten Eltern zu einer Verstärkung ihres subjektiven Belastungserlebens. Die Zusammenhänge zwischen dem Fragebogen zur Belastung durch die Betreuung und den Schlafstörungen der betreuenden Kinder sind zwar gering, eine schlechte allgemeine Schlafqualität steht bei ihnen allerdings in einem eindeutigen Zusammenhang mit Einschränkungen in den physischen und psychischen Bereichen der SF-36. Diese dienen als indirektes Maß für die subjektive Belastung der erwachsenen Kinder durch die Betreuung ihrer erkrankten Eltern. Besonders häufig finden sich hierbei Verbindungen mit der „körperlichen Funktionsfähigkeit“ und „Rollenfunktion“ sowie mit der „Vitalität" und der ,allgemeinen Gesundheit“ der Studienteilnehmer. Wie nicht anders erwartet, sind es auch hier wieder die Töchter, die durch Schlafstörungen in ihrem subjektiven Belastungserleben stärker beeinflusst werden als die Söhne. Eine signifikante Korrelation zwischen der Tagesschläfrigkeit und dem Belastungserleben der betreuenden Kinder konnte hingegen nicht bestätigt werden. 
Bezüglich der Gruppe der Parkinsonpatienten kann die dritte Hypothese vollends bestätigt werden: Sowohl eine erhöhte Tagesschläfrigkeit als auch eine eingeschränkte allgemeine Schlafqualität stehen in einem eindeutigen Zusammenhang mit Einschränkungen in der gesundheitsbezogenen Lebensqualität. Besonders häufig und daher hervorzuheben sind hierbei die Korrelationen mit den Dimensionen „körperliche Funktionsfähigkeit“, „körperliche Schmerzen“, ,allgemeine Gesundheit“, „Vitalität“ und „psychisches Wohlbefinden“. Darüber hinaus erwähnenswert ist der Befund, dass die anhand der PDSS gemessenen Schlafstörungen der Parkinsonpatienten mit ihrer subjektiven Belastung in einer sehr viel engeren Verbindung stehen als ihre mit Hilfe der PSQI ermittelte allgemeine Schlafqualität.

\subsection{Diskussion Hypothese 4}

Die zur Überprüfung der vierten Hypothese berechneten hohen Korrelationen unterstützen eindeutig die Annahme, dass sich eine hohe Pflegebedürftigkeit der Parkinsonpatienten negativ auf das Belastungserleben ihrer betreuenden erwachsenen Kinder auswirkt. Eine hohe elterliche Pflegebedürftigkeit hat demnach neben einem stark negativen direkten Effekt auf die subjektiv empfundene Belastung auch indirekt via Einschränkungen im Bereich der gesundheitsbezogenen Lebensqualität der betreuenden Kinder einen Einfluss auf deren Belastungserleben. Zudem scheint ein Zusammenhang zwischen dem Grad der Pflegebedürftigkeit der Eltern und der Prävalenz depressiver Symptome bei den erwachsenen Kindern zu bestehen. Aufgrund der fehlenden Signifikanz kann dies allerdings nur als Trend gewertet werden. Die Erkenntnisse dieser Untersuchung werden durch Forschungsergebnisse verschiedener weiterer Autoren gestützt. Sowohl Vitaliano und Kollegen als auch Thommessen und Mitarbeiter wiesen eine Verbindung zwischen funktionellen Defiziten der pflegebedürftigen Person und der subjektiven Belastung des betreuenden Familienangehörigen nach (Thommessen et al. 2002; Vitaliano et al. 2004). Zudem ist die Beeinträchtigung der Alltagsfähigkeit von Parkinsonpatienten laut Glozman mitunter hauptursächlich für die reduzierte Lebensqualität ihrer betreuenden Angehörigen (Glozman 2004). Martínez-Martín und Mitarbeiter sehen hierbei insbesondere die mentalen Aspekte der gesundheitsbezogenen Lebensqualität der betreuenden Angehörigen eingeschränkt (Martínez-Martín et al. 2007). In diesem Zusammenhang interessant ist daher auch das Ergebnis der vorliegenden Untersuchung, nach der sich der Grad der elterlichen Pflegebedürftigkeit lediglich auf die psychosozialen Lebensqualitätsbereiche ihrer betreuenden Kinder „soziale Funktionsfähigkeit“ und „emotionale Rollenfunktion“ und nicht auch auf die körperlichen Bereiche ihrer gesundheitsbezogenen Lebensqualität negativ auswirkt. Als mögliche Erklärung könnte folgender Sachverhalt dienen: Die Betreuung des erkrankten Elternteils steht in zeitlicher Konkurrenz $\mathrm{zu}$ anderen Verpflichtungen und sozialen Rollen der erwachsenen Kinder, was aufgrund der zunehmenden zeitlichen Beanspruchung durch die Pflege die frei verfügbare Zeit der pflegenden 
Angehörigen minimiert. Dies führt zu Einschränkungen der Sozialkontakte und könnte somit eine Beeinträchtigung der psychosozialen Lebensqualität der betreuenden Söhne und Töchter bewirken (vgl. Adler et al. 1996; Brody 1985; Schütze und Wagner 1991; Thommessen et al. 2002). Auch in der 2006 veröffentlichten Untersuchung von Schrag et al. berichten zwei Drittel der pflegenden Partner von Parkinsonpatienten von durch die Pflegesituation bedingten Einschränkungen ihres sozialen Lebens. Fünfundzwanzig Prozent gaben zudem an, dass ihre Kontakte zu anderen Familienmitgliedern unter der Pflege litten (Schrag et a. 2006). Shin und Kollegen berichten ebenfalls davon, dass das Belastungserleben pflegender Kinder von Parkinsonpatienten stark von der empfundenen sozialen Unterstützung durch Privatpersonen und professioneller Stelle abhängt (Shin et al. 2012).

Weiterhin sind die erkrankten Parkinsonpatienten der vorliegenden Untersuchung im Vergleich mit den Studienpopulationen der Voruntersuchungen von Stiens (2000), Linkersdörfer (2006) und Schmidt (2009) nicht nur wesentlich jünger - und damit auch ihre betreuenden erwachsenen Kinder sondern sind aufgrund ihrer vergleichsweise geringeren krankheitsbedingten Einschränkungen auch noch in einem wesentlich geringeren Umfang hilfs- bzw. pflegebedürftig. Aufgrund dieser verhältnismäßig noch moderaten instrumentellen Abhängigkeit der Eltern könnte sich die körperliche Belastung der betreuenden Kinder der aktuellen Untersuchung daher noch in einem vertretbaren Rahmen halten. Die Einhaltung ihrer körperlichen Belastungsgrenze könnte so den ausbleibenden Effekt der elterlichen Pflegebedürftigkeit auf die körperlichen Dimensionen ihrer gesundheitsbezogenen Lebensqualität erklären.

Die Pflegebedürftigkeit der Patienten und die damit verbundene Belastung der betreuenden Söhne und Töchter stehen weiterhin in engem Zusammenhang mit der anhand der UPDRS-Motorskala gemessenen Schwere der Symptome, dem Erkrankungsstadium nach Hoehn und Yahr und der Pflegestufe der Patienten. Eine ausgeprägte Symptomatik, ein fortgeschrittenes H\&Y-Stadium sowie eine hohe Pflegestufe führen demnach zu einer vergleichsweise hohen Pflegebedürftigkeit der erkrankten Eltern und bewirken bei den betreuenden Kindern folglich eine erhöhte Belastung. Dies steht im Einklang mit Forschungsergebnissen von Kim und Kollegen, denen zufolge die subjektive Belastung betreuender Angehöriger von Parkinsonpatienten in Abhängigkeit von dem Ausmaß der motorischen Beeinträchtigung und der Höhe des Krankheitsstadiums der Patienten ansteigt (Kim et al. 2007). Razali und Mitarbeiter sowie Abendroth et al. wiesen ebenfalls einen negativen Einfluss der Schwere der Erkrankung auf die Pflegebelastung nach (Abendroth et al. 2011; Razali et al. 2012). In beiden Studien hing das Ausmaß der Belastung zudem mit der Dauer der geleisteten Pflege zusammen.

Darüber hinaus steigt in der vorliegenden Untersuchung die Pflegebelastung der betreuenden Söhne und Töchter mit zunehmender Verschlechterung der elterlichen Lebensqualität in den körperlichen Bereichen der SF-36 an. Eine verminderte elterliche „Vitalität“ und „körperliche Funktionsfähigkeit“ 
scheinen hierbei führende Belastungsfaktoren zu sein. Des Weiteren fühlen sich die erwachsenen Kinder durch eine depressive Komorbidität der Eltern belastet, die Töchter darüber hinaus durch ein reduziertes „psychisches Wohlbefinden“ ihrer Eltern. In der Literatur lassen sich sowohl Hinweise auf einen Zusammenhang des Belastungserlebens betreuender Angehöriger mit körperlichen als auch psychischen Faktoren der betreuten Personen finden (vgl. Caap-Ahlgren und Dehlin 2002; Chappell und Reid 2002; England und Tripp-Reimer 2003; Lökk 2008; Martínez-Martín et al. 2008; Molyneux et al. 2008; Shin et al. 2012). Mentale Symptome der Parkinsonpatienten wie Depressionen, Verwirrung und Sinnestäuschungen spielten hierbei eine besonders wichtige Rolle für das Ausmaß des subjektiven Belastungserlebens ihrer betreuenden Ehepartner und erwachsenen Kinder (Årsland et al. 1999; Hooker et al. 2000; Lökk 2008; Schrag et al. 2006; Thommessen et al. 2002). Ergebnissen von Schrag und Mitarbeitern zufolge steht die Pflegebelastung nicht nur im Zusammenhang mit dem Vorhandensein depressiver Symptome bei Pflegenden und Patienten sowie deren Einschränkung der Lebensqualität sondern auch mit der Zufriedenheit der Pflegenden über die eigene eheliche und sexuelle Beziehung (Schrag et al. 2006). Der negative Einfluss auf die jugendlichen und erwachsenen Kinder der Parkinsonpatienten steigt zudem mit zunehmender Krankheitsdauer (Schrag et al. 2004). Ein in die gleiche Richtung weisender Trend konnte auch in der aktuellen Untersuchung festgestellt werden und wird durch weitere Forschungsergebnisse u.a. von Caap-Ahlgren und Dehlin sowie Martínez-Martín und Mitarbeitern bestätigt (Caap-Ahlgren und Dehlin 2002; Martínez-Martín et al. 2008). Das Belastungserleben jugendlicher und erwachsener Kinder von Parkinsonpatienten mit einem frühen Krankheitsbeginn hängt darüber hinaus mit einem Mangel an Informationen über die Erkrankung des Elternteils und deren Verlauf zusammen (Schrag et al. 2004).

Auch bei der Bearbeitung der vierten Hypothese fielen deutliche geschlechtsspezifische Unterschiede auf: Die Töchter werden von dem Grad der elterlichen Hilfs- und Pflegebedürftigkeit wesentlich stärker beeinflusst als die betreuenden Söhne. Gründe hierfür könnten zum einen in der allgemein stärkeren Belastung der weiblichen Studienteilnehmer liegen (vgl. 4.2.4), was per se mit einer erhöhten Chance signifikanter Korrelationen einhergeht. Andererseits könnte dieser Sachverhalt sicherlich auch mit der größeren Mühelosigkeit von Frauen, eigene Gefühle auszudrücken und sie bei anderen wahrzunehmen, zusammenhängen.

\subsubsection{Zusammenfassung Diskussion Hypothese 4}

Die vierte Hypothese kann anhand der Ergebnisse der vorliegenden Untersuchung somit eindeutig bestätigt werden: Die Pflegebedürftigkeit der Parkinsonpatienten korreliert positiv mit dem Belastungserleben der betreuenden Kinder. Zusammenfassend kann festgehalten werden, dass sich eine hohe Hilfsbedürftigkeit der Eltern nicht nur direkt auf die subjektive Belastung der betreuenden Kinder sondern auch auf die psychosozialen Bereiche ihrer Lebensqualität auswirkt. Zudem scheint ihr Belastungsempfinden in einem engen Zusammenhang mit dem Ausmaß der körperlichen 
Einschränkungen ihrer hilfsbedürftigen Eltern zu stehen. Der Effekt auf die betreuenden Töchter ist hierbei abermals eindeutiger als der auf die Söhne.

\subsection{Diskussion Hypothese 5}

Die fünfte Hypothese lautete: Eine hohe filiale Reife und eine gute Schlafqualität korrelieren positiv miteinander. Auf der Basis der aktuellen Untersuchungsergebnisse kann diese Aussage nicht in vollem Umfang bestätigt werden. Das Ausmaß der filialen Angst bzw. Reife der Studienteilnehmer beeinflusst demnach weder ihre mittels der Epworth Sleepiness Scale (ESS) gemessene Tagesschläfrigkeit noch ihre allgemeine Schlafqualität, die mit Hilfe des Pittsburgh Sleep Quality Index (PSQI-Gesamtwert) erhoben wurde. Es zeigen sich lediglich einige schwache Zusammenhänge zwischen einzelnen Unterskalen des Pittsburgh Sleep Quality Index (PSQI) und der Filial Anxiety Scale (FAS) bzw. den Rubriken des Filiale-Reife-Bogens (LFMS-A).

Positive Korrelationen, die das geforderte Signifikanzniveau von $\mathrm{p}<0,05$ erreichen, finden sich zwischen der Filialen Angst A und der PSQI-Unterskala „Schlafstörungen“. Eine relativ große Sorge der erwachsenen Kinder über die eigene Fähigkeit, der erwarteten Hilfsbedürftigkeit der alternden Eltern entsprechen zu können, scheint demnach bei den betreuenden Kindern häufiger Schlafstörungen zur Folge zu haben. Ein in die gleiche Richtung weisender Trend zeigt sich zudem bezüglich der „subjektiven Schlafqualität“. Diese Zusammenhänge verwundern nicht weiter. So ist es naheliegend, dass ein betreuendes erwachsenes Kind, welches sich aus gegebenem Anlass um die eigene Fähigkeit zur Pflege sorgt und diese eventuell als unzureichend empfindet, vermehrt mit Schlafstörungen zu kämpfen hat, was in der Folge mit einer Verschlechterung seiner subjektiven Schlafqualität einher geht.

Unverständlich erscheint hingegen folgendes Ergebnis der aktuellen Untersuchung, bei dem es sich allerdings nur um einen nicht-signifikanten Trend handelt: Eine hohe filiale Angst A scheint mit einer guten Schlafdauer der Studienteilnehmer in Verbindung zu stehen. Gründe für diese Trends konnten anhand der aktuellen Ergebnisse nicht eruiert werden. Auch finden sie keinerlei Bestätigung in der diesbezüglichen internationalen Fachliteratur. Es ist demnach anzunehmen, dass es sich hierbei um ein Artefakt handelt.

Wiederum sinnvoll erscheinen die positiven Korrelationen zwischen der filialen Angst B der Studienteilnehmer und dem Ausmaß ihrer Schlafstörungen und der allgemeinen Schlafeffizienz der Töchter. Den Ergebnissen der vorliegenden Studie zufolge leiden betreuende Kinder, die sich vermehrt um den abnehmenden gesundheitlichen Zustand ihrer erkrankten Eltern und ihrer damit verbundenen zunehmenden Hilfsbedürftigkeit sorgen, häufiger unter Schlafstörungen und einer verminderten Schlafeffizienz als betreuende Kinder mit einer geringeren filialen Angst B. 
Wie bereits in Kapitel 1.2.2.1 erwähnt, haben die betreuenden erwachsenen Kinder der Parkinsonpatienten nicht nur die Rolle als Pflegeleistender inne, sondern sehen sich darüber hinaus in ihrem Privat- und Berufsleben auch mit der Erfüllung diverser anderer sozialer Rollen wie beispielsweise Mutter, Vater, Partner, Freund, Freundin oder Kollege konfrontiert. Es könnte daher sein, dass ihnen tagsüber daher häufig wenig Zeit bleibt, sich mit den Sorgen und Ängsten bezüglich der alternden Eltern auseinander zu setzen. So könnten es vorwiegend die ruhigeren Abend- und Nachtstunden sein, in denen sich die betreuenden Kinder - bewusst und unbewusst - mit ihrer filialen Angst auseinandersetzen. Sei es nun die Sorge um die eigenen Fähigkeiten und Grenzen bezüglich der Pflegeübernahme oder sei es die direkte Sorge um die schwindende elterliche Gesundheit und die damit in naher Zukunft zunehmende Hilfsbedürftigkeit der Eltern. Ein erhöhtes Maß an Schlafstörungen sowie eine verminderte Schlafeffizienz und eine schlechtere subjektive Schlafqualität der betreuenden erwachsenen Kinder könnten die Folge sein.

Von den sieben Faktoren der filialen Reife sind es das „filiale Verpflichtungsgefühl“, die „filiale Hilfe“ und die „familiäre Solidarität", die einen gewissen Zusammenhang mit dem Schlafverhalten der Studienteilnehmer erkennen lassen. So führt ein hohes filiales Verpflichtungsgefühl zu häufigen Schlafstörungen der betreuenden Kinder und zu einer verminderten subjektiven Schlafqualität der Töchter. Ein übermäßig starkes Verpflichtungsgefühl der erwachsenen Kinder gegenüber ihren hilfsbedürftigen Eltern gilt als Hinweis auf ein filial unreifes Verhalten (vgl. Kapitel 1.2.3, 4.2.3 und 4.4). Es erscheint allerdings unwahrscheinlich, dass ein hohes filiales Verpflichtungsgefühl per se mit einer erhöhten Frequenz nächtlicher Schlafstörungen der betreuenden Kinder einhergeht. Daher sollte folgender Sachverhalt bedacht werden: Wie in den vorangegangenen Kapiteln bereits ausführlich diskutiert, steht ein verhältnismäßig hohes filiales Verpflichtungsgefühl in einem engen Zusammenhang mit einer erhöhten subjektiven Belastung der erwachsenen Kinder durch die Betreuung ihrer erkrankten Eltern. Eine hohe Pflegebelastung ist wiederum eng mit einer allgemein eingeschränkten Schlafqualität der Betroffenen verbunden. So könnte das Ausmaß des subjektiven Belastungserlebens als vermittelnder Faktor zwischen dem filialen Verpflichtungsgefühl und den Schlafstörungen des Studienkollektivs fungieren. Die filiale Reife an sich hätte somit keinen direkten sondern lediglich einen indirekten Einfluss auf die Schlafqualität der betreuenden erwachsenen Kinder. Zur Bestätigung dieser These bedarf es sicherlich weiterer gezielter Untersuchungen.

Anhand der vorliegenden Ergebnisse sind weiterhin statistische Zusammenhänge zwischen einer hohen „filialen Hilfe“ und einer erhöhten Tagesschläfrigkeit sowie einer erhöhten Frequenz an Schlafstörungen festzustellen. Bei letzterem handelt es sich allerdings lediglich um einen Trend ( $p=0,057$ ). Darüber hinaus scheint sich eine als gut empfundene ,familiäre Solidarität“ positiv auf die Schlafqualität der betreuenden Kinder auszuwirken. So berichten beispielsweise diejenigen, die in ihrer Pflege- bzw. Betreuungsrolle ausreichend Unterstützung durch andere Familienmitglieder 
erfahren, von einer besseren Schlafeffizienz als jene betreuenden Kinder aus eher „unsolidarischen“ Familien. Die Töchter berichten zudem im Zusammenhang mit einer guten Familiensolidarität von einer durchschnittlich besseren subjektiven Schlafqualität. So bestätigt sich auch hier wieder die protektive Wirkung des Reife-Faktors „familiäre Solidarität“. Zu diskutieren ist jedoch sicherlich auch hier, ob es sich dabei tatsächlich um einen direkten Effekt der Familiensolidarität auf das Schlafverhalten der betreuenden Kinder handelt, oder ob die dargestellten Zusammenhänge doch eher wieder über die Mediatoren ,allgemeine Belastung durch die Betreuung“ und die Komorbidität Depressivität zustande kommen.

Abschließend soll noch einmal näher auf das Fehlen signifikanter Korrelationen der Tagesschläfrigkeit (ESS) und der allgemeinen Schlafqualität (PSQI-Gesamtwert) der betreuenden erwachsenen Kinder eingegangen werden. Wie bereits in Kapitel 4.2.7 dargestellt, unterscheiden sich die betreuenden Söhne und Töchter der aktuellen Untersuchung weder bezüglich ihrer Tagesschläfrigkeit noch in ihrer allgemeinen Schlafqualität von den gesunden Referenzgruppen der Originalstudien (vgl. Bloch et al. 1999; Zeitlhofer et al. 2000). Auch im Vergleich mit den alters- und geschlechtsentsprechenden Kontrollpersonen der aktuellen Untersuchung sind keine signifikanten Unterschiede im Schlafverhalten festzustellen. Leiden die betreuenden erwachsenen Kinder der vorliegenden Untersuchung vielleicht nicht genug unter Schlafstörungen, um tatsächlich signifikante Effekte bezüglich der Ausprägung ihrer filialen Reife festzustellen? Auch scheint das Schlafverhalten der Probanden ihre subjektiv empfundene Belastung durch die Betreuung nicht sonderlich zu beeinflussen (vgl. Kapitel 4.6).

Dieser Überlegung gegenüber steht allerdings die Tatsache, dass die relativ geringen Einschränkungen, die die Studienteilnehmer durch ihre allgemeine Schlafqualität erfahren, in deutlich signifikantem Zusammenhang mit den verschiedenen Dimensionen ihrer gesundheitsbezogenen Lebensqualität stehen, die als Maß für die physischen und psychischen Belastungen innerhalb des Betreuungsverhältnisses anzusehen sind. Hierfür scheinen die geringe Tagesschläfrigkeit und die allenfalls tendenziell schlechtere allgemeine Schlafqualität der betreuenden Söhne und Töchter also ,auszureichen“.

Ungeachtet dessen erscheint es ratsam, eine erneute Untersuchung dieser Fragegestellung anzustreben. Die zukünftigen Studienkollektive sollten zu diesem Zweck nicht nur eine insgesamt größere Teilnehmerzahl aufweisen. Es wäre ebenfalls wünschenswert, wenn sich diese betreuenden erwachsenen Kinder bezüglich ihres Schlafverhaltens signifikant von entsprechenden Kontrollpersonen unterschieden. Auf diese Weise könnte der fehlende Effekt vermehrter Schlafstörungen der Studienteilnehmer auf den Zusammenhang zwischen filialer Reife und allgemeiner Schlafqualität - wie es in der aktuellen Untersuchung der Fall ist - umgegangen werden. 


\subsubsection{Zusammenfassung Diskussion Hypothese 5}

Zusammenfassend kann demnach festgehalten werden, dass sich anhand der vorliegenden Untersuchungsergebnisse die Aussage der fünften Hypothese nicht bestätigen lässt. So steht weder die Epworth Sleepiness Scale (ESS) als Maß für die Tagesschläfrigkeit der Studienteilnehmer noch der Gesamtwert des Pittsburgh Sleep Quality Index (PSQI), anhand dessen die allgemeine Schlafqualität des Kollektivs erhoben wurde, in einem signifikanten Zusammenhang mit dem Grad der filialen Angst bzw. Reife der erwachsenen Kinder, die sich in der Betreuung ihrer an dem idiopathischen Parkinsonsyndrom erkrankten Eltern engagieren. Es lassen sich diesbezüglich lediglich einige schwache Korrelationen zwischen einzelnen Unterskalen des PSQI und der Filial Anxiety Scale (FAS) bzw. den Items des Filiale-Reife-Bogens (LFMS-A) feststellen. Wesentliche Hinweise auf einen direkten Zusammenhang zwischen der Ausprägung der filialen Reife und dem Schlafverhalten der betreuenden Kinder ergeben sich aus der aktuellen Datenlage daher nicht. 


\section{Zusammenfassung}

Das Ziel der vorliegenden Untersuchung bestand darin, eine aussagekräftige und effektive Skala zur Messung der Filialen Reife pflegender Angehöriger zu finden. Im Mittelpunkt standen hierzu die Louvain Filial Maturity Scale A nach Marcoen (LFMS-A) und die Filial Anxiety Scale nach Cicirelli (FAS). Diese wurden erwachsenen betreuenden Kindern von Parkinsonpatienten vorgelegt, um Zusammenhänge zwischen dem Grad ihrer filialen Reife bzw. Angst und dem Ausmaß ihres subjektiven Belastungserlebens zu ermitteln. Besonderes Augenmerk wurde darauf gelegt, welche der Skalen in einem engeren Zusammenhang mit der Belastung der betreuenden Söhne und Töchter stand und folglich eine größere Relevanz in der Beziehung zwischen hilfsbedürftigen Eltern und ihren erwachsenen Kindern aufweist. Darüber hinaus wurde der Frage nachgegangen, inwieweit sich Belastungs- und Beziehungserleben auf die subjektive Schlafqualität der Studienteilnehmer auswirken. Die Ergebnisse dieser Studie sollen dazu beitragen, das Konzept der Filialen Reife langfristig in der Angehörigenberatung zu etablieren, um Risikopersonen besser beraten und Copingstrategien für einen gelungenen Umgang mit pflegebedürftigen Familienmitgliedern gezielter fördern zu können.

Die Stichprobe dieser Untersuchung setzte sich aus 50 Patienten-Betreuer-Paaren und 50 Kontrollpersonen zusammen, die mit Hilfe standardisierter Fragebögen getrennt voneinander interviewt wurden. Bei den Patienten handelte es sich um Männer und Frauen im Alter von 52 bis 84 Jahren, die an einem idiopathischen Parkinsonsyndrom erkrankt waren. Als Betreuer wurde jeweils ein erwachsenes Kind der Patienten befragt, welches sich in der Betreuung des erkrankten Elternteils engagierte. Die Gruppe der Kontrollpersonen setzte sich aus erwachsenen Söhnen und Töchtern von gesunden alten Menschen zusammen, die im Vorfeld entsprechend Alter, Geschlecht und Bildungsbzw. Berufsstand auf die Betreuergruppe abgestimmt wurden. Zu Beginn der Befragung wurde ein halbstandardisiertes Interview zur Anamneseerhebung und zur näheren Erfassung des Umfelds der Betreuung sowie der familiären Situation der Patienten und der betreuenden Kinder bzw. der Kontrollpersonen durchgeführt. Der Fragenkatalog für die Betreuer- und Kontrollgruppe umfasste neben den beiden bereits erwähnten Fragebögen zur filialen Reife und Angst, Louvain Filial Maturity Scale A nach Marcoen (Filiale-Reife-Skala A) und die Filial Anxiety Scale nach Cicirelli (FilialeAngst-Skala), einen auf dem Zarit-Burden-Interview basierenden Fragebogen zur Belastung durch die Betreuung, den Short-Form-36 Health Survey (SF-36) zur Erfassung der subjektiv empfundenen gesundheitsbezogenen Lebensqualität der Studienteilnehmer, die 10-Item Center for Epidemiological Studies Depression Scale (CESD-10) zur Messung der Depressivität sowie die Epworth Sleepiness Scale (ESS) zur Bestimmung der Tagesschläfrigkeit und den Pittsburgh Sleep Quality Index (PSQI) zur Beurteilung der allgemeinen Schlafqualität der Probanden. Die Parkinsonpatienten füllten bezüglich ihrer allgemeinen Schlafqualität zudem die Parkinson Disease Sleepiness Scale (PDSS) aus. Zur Objektivierung der Pflegebedürftigkeit der Parkinsonpatienten bzw. der gesunden Eltern der 
Kontrollpersonen wurde zudem die Nurses' Observation Scale for Geriatric Patients (NOSGER II) eingesetzt. Die Ergebnisse der Studiengruppe wurden anschließend sowohl im Vergleich mit den Referenzwerten der Originalstudien als auch mit denen der Kontrollgruppe beurteilt. Zudem wurden zur Darstellung der fünf Untersuchungshypothesen Korrelationen zwischen verschiedenen Werten bestimmter Fragebögen errechnet.

So konnte anhand der vorliegenden Ergebnisse gezeigt werden, dass ein enger Zusammenhang zwischen der filialen Angst bzw. Reife erwachsener Kinder hilfsbedürftiger Eltern und deren subjektivem Belastungserleben besteht (Hypothese 1). Die an dieser Untersuchung teilnehmenden erwachsenen Kinder von Parkinsonpatienten werden von einer verhältnismäßig hohen filialen Angst sowie einem geringen Grad filialer Reife in der Betreuungssituation eingeschränkt, was sich in einer Verstärkung ihrer subjektiven Belastung widerspiegelt. Die Sorge der betreuenden Kinder vor dem erwarteten zukünftigen Nachlassen des körperlichen und geistigen Zustandes des erkrankten Elternteils (filiale Angst B) scheint dabei interessanterweise einen stärkeren Einfluss auf die empfundene Belastung zu haben als ihre Angst bezüglich der eigenen Fähigkeiten, der erwarteten Rolle als pflegende Personen gerecht zu werden (filiale Angst A). Die sieben Faktoren der filialen Reife zeigen ebenfalls unterschiedlich starke Assoziationen mit dem Belastungserleben. So wirkt sich insbesondere ein starkes „filiales Verpflichtungsgefühl“ negativ auf die Belastung der betreuenden Kinder aus. Eine verhältnismäßig große „filiale Autonomie“ der erwachsenen Kinder sowie eine als positiv empfundene „familiäre Solidarität“ scheinen zudem eine deutlich protektive Wirkung zu haben. Hingegen scheint sich der Einfluss der „filialen Liebe“ und der „elterlichen Besorgnis“ auf das psychische Wohlbefinden der betreuenden Kinder zu beschränken, und die Dimensionen „filiale Hilfe“ und „filiale Hilfsbereitschaft" scheinen insgesamt keinen nennenswerten Einfluss auf das Belastungserleben der Studienteilnehmer zu haben.

Zudem bestätigt sich die zweite Hypothese dieser Untersuchung: Die mittels der FAS gemessene filiale Angst der betreuenden erwachsenen Kinder der Parkinsonpatienten steht in einem engeren Zusammenhang mit ihrer subjektiv empfundenen Belastung als ihre anhand der LFMS-A erhobene filiale Reife. Die Filial Anxiety Scale nach Cicirelli scheint demnach besser geeignet zu sein, anhand des Ausmaßes der filialen Angst die Situation der betreuenden erwachsenen Kinder innerhalb der Pflege zu erfassen als der Filiale-Reife-Bogen nach Marcoen. Aufgrund der größeren Anwendungsfreundlichkeit der Filial Anxiety Scale ergeben sich hieraus auch positive Konsequenzen für den klinischen Einsatz des Fragebogens und die Beratung pflegender Angehöriger in der Praxis.

Hinsichtlich der allgemeinen Schlafqualität der betreuenden Söhne und Töchter weisen die aktuellen Ergebnisse auf deutliche Zusammenhänge mit ihrer gesundheitsbezogenen Lebensqualität hin (1. Teil Hypothese 3). Insbesondere eine als schlecht empfundene „körperlichen Funktionsfähigkeit“ und 
„Rollenfunktion“ der Studienteilnehmer sowie Einschränkungen in den Bereichen „Vitalität“ und „allgemeine Gesundheit“ gehen mit einer Verminderung ihrer allgemeinen Schlafqualität und einem gehäuftem Auftreten von Schlafstörungen einher. Signifikante Korrelationen zwischen der Tagesschläfrigkeit und dem Belastungserleben der betreuenden Kinder konnten hingegen nicht festgestellt werden.

Der zweite Teil der dritten Hypothese kann vollends bestätigt werden: Sowohl eine erhöhte Tagesschläfrigkeit als auch eine eingeschränkte allgemeine Schlafqualität der Parkinsonpatienten stehen in einem eindeutigen Zusammenhang mit Einschränkungen in ihrer gesundheitsbezogenen Lebensqualität. Den Dimensionen „körperliche Funktionsfähigkeit“, „körperliche Schmerzen“, „allgemeine Gesundheit“, „Vitalität“ und „psychisches Wohlbefinden“ scheint hierbei eine besondere Rolle zuzukommen.

Darüber hinaus konnte mit Hilfe der aktuellen Untersuchungsergebnisse die Verbindung zwischen dem Grad der Pflegebedürftigkeit der Parkinsonpatienten und dem Belastungserleben ihrer betreuenden Söhne und Töchter eindeutig gezeigt werden (Hypothese 4). Eine hohe elterliche Hilfsbedürftigkeit hat demnach neben einem stark negativen direkten Effekt auf die subjektiv empfundene Belastung auch via Einschränkungen in den psychosozialen Bereichen der gesundheitsbezogenen Lebensqualität der betreuenden Kinder einen indirekten Einfluss auf ihr Belastungserleben. Eine hohe Pflegebedürftigkeit der erkrankten Eltern und die damit verbundene Belastung ihrer Kinder sind dabei maßgeblich mit einer ausgeprägteren Symptomatik, einem fortgeschrittenen Erkrankungsstadium nach Hoehn und Yahr sowie einer hohen Pflegestufe der Patienten verbunden. Weiterhin scheint das „kindliche“ Belastungsempfinden in einem engen Zusammenhang mit dem Ausmaß der körperlichen Einschränkungen ihrer hilfsbedürftigen Eltern zu stehen.

Einzig die fünfte Hypothese, laut der der Grad der filialen Reife in einem direkten Zusammenhang mit dem Schlafverhalten der Studienteilnehmer steht, konnte im Rahmen der hier vorliegenden Untersuchung nicht bestätigt werden. So steht weder die Epworth Sleepiness Scale (ESS) als Maß für die Tagesschläfrigkeit der Studienteilnehmer noch der Gesamtwert des Pittsburgh Sleep Quality Index (PSQI), anhand dessen die allgemeine Schlafqualität des Kollektivs erhoben wurde, in einem signifikanten Zusammenhang mit dem Grad der filialen Angst bzw. Reife der erwachsenen Kinder, die sich in der Betreuung ihrer an dem idiopathischen Parkinsonsyndrom erkrankten Eltern engagieren.

Erwähnenswert sind die in der vorliegenden Studie aufgetretenen Geschlechtsunterschiede der betreuenden erwachsenen Kinder hinsichtlich der Ausprägung ihrer filialen Reife und dem Ausmaß des subjektiven Belastungserlebens. So sind es die Töchter, die im Vergleich eine allgemein höhere filiale Angst sowie ein höheres filiales Verpflichtungsgefühl und eine geringere filiale Autonomie 
aufweisen als die männlichen Studienteilnehmer und damit einen schlechteren Ausgangspunkt für einen erfolgreichen Umgang mit der Pflegesituation haben. Auch scheinen die Töchter eine größere subjektive Belastung durch die Betreuung ihrer Eltern $\mathrm{zu}$ erfahren. Ihre gesundheitsbezogene Lebensqualität, ihr psychisches Wohlergehen und ihre allgemeine Schlafqualität bleiben ebenfalls hinter den entsprechenden Angaben der betreuenden Söhne zurück.

Zusammenfassend kann festgestellt werden, dass das Ziel dieser Untersuchung erreicht wurde: Es konnten weitere Prädiktoren für einen erfolgreichen Umgang mit der häuslichen Pflege bzw. Betreuung eines erkrankten Familienangehörigen beschrieben werden. Darüber hinaus scheint die Erfassung der filialen Angst im Zusammenhang mit dem Belastungserleben erwachsener Kinder von Parkinsonpatienten relevanter zu sein als das Erfassen ihrer filialen Reife. 


\section{Abbildungen}

Abb. 1: Ergebnisse GDS cut-off (Patienten)

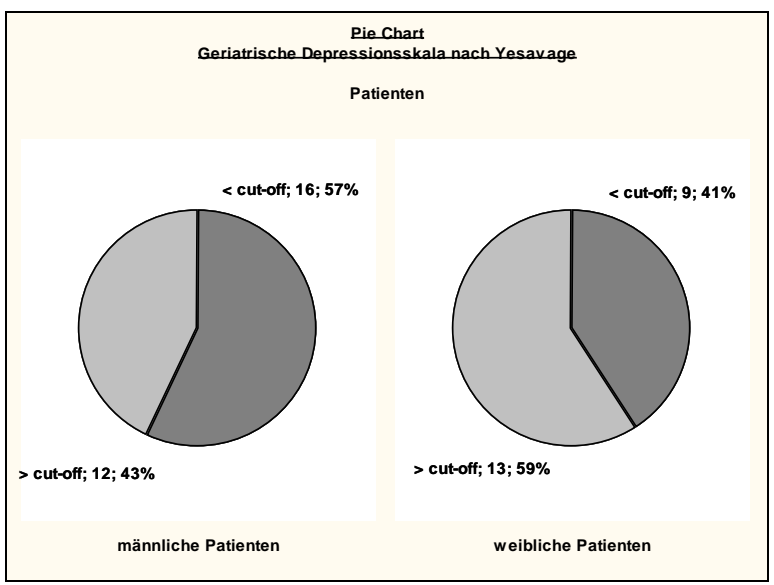

Abb. 3: Ergebnisse CESD-10 (Patienten)

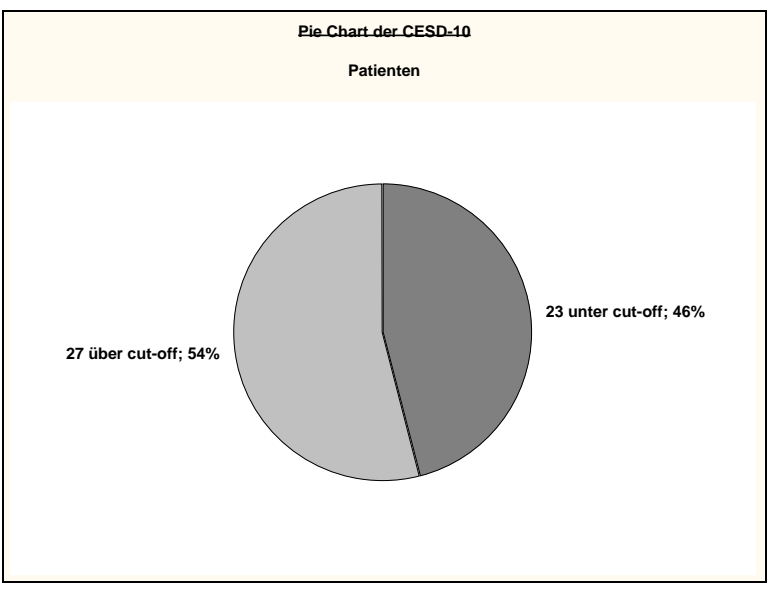

Abb. 5: Ergebnisse SF-36 (Patienten)

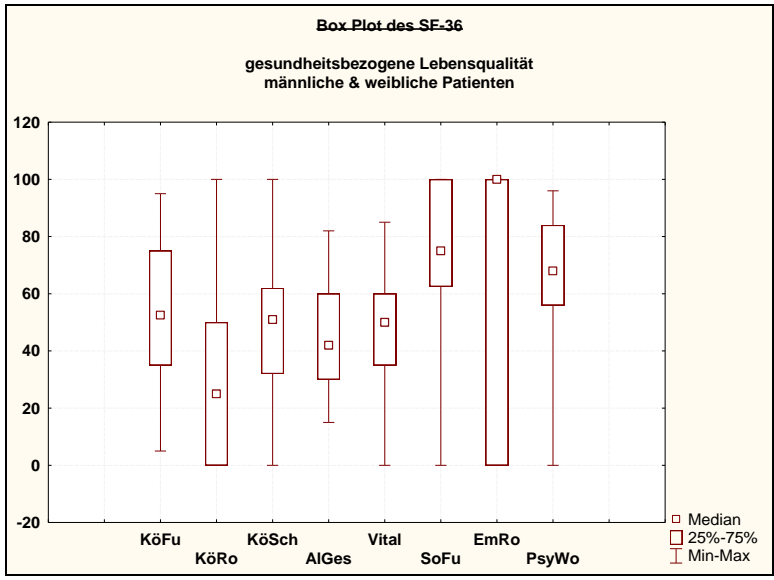

Abb. 2: Ergebnisse GDS (Patienten)

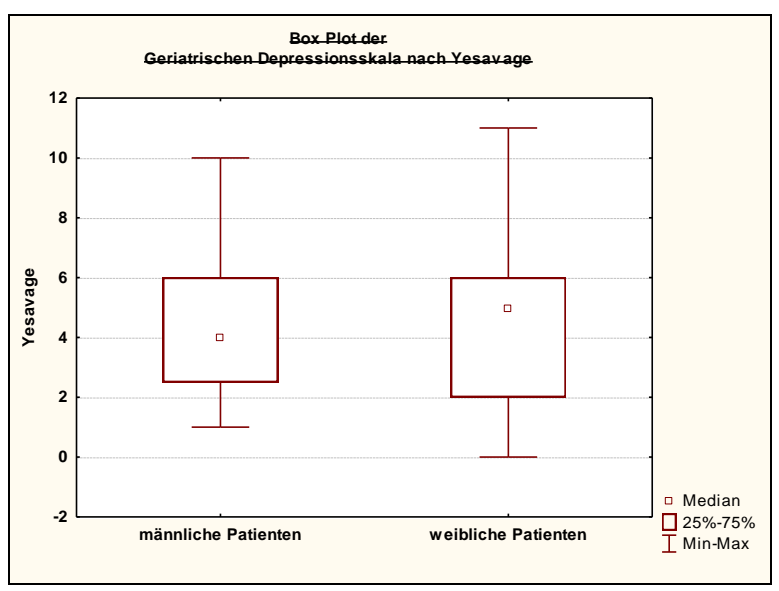

Abb. 4: Ergebnisse CESD-10 (männl./ weibl. Patienten)

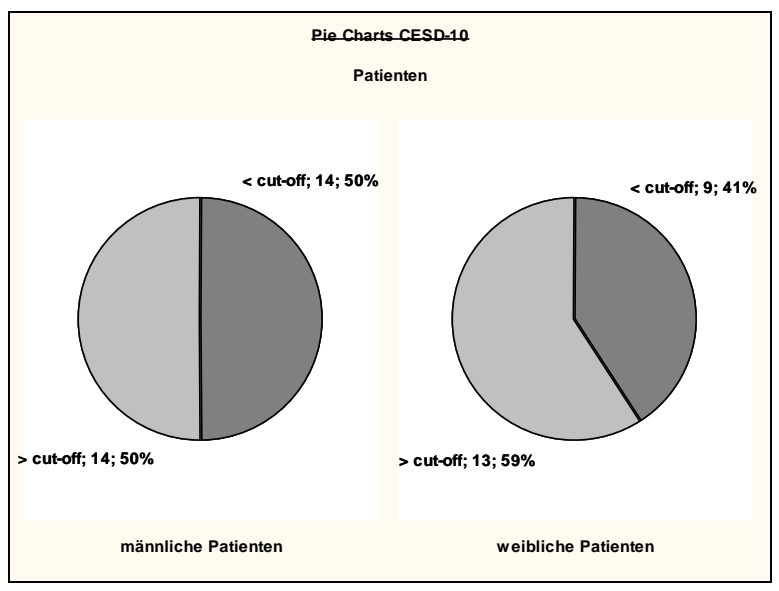

Abb. 6: Ergebnisse SF-36 (männl. Patienten)

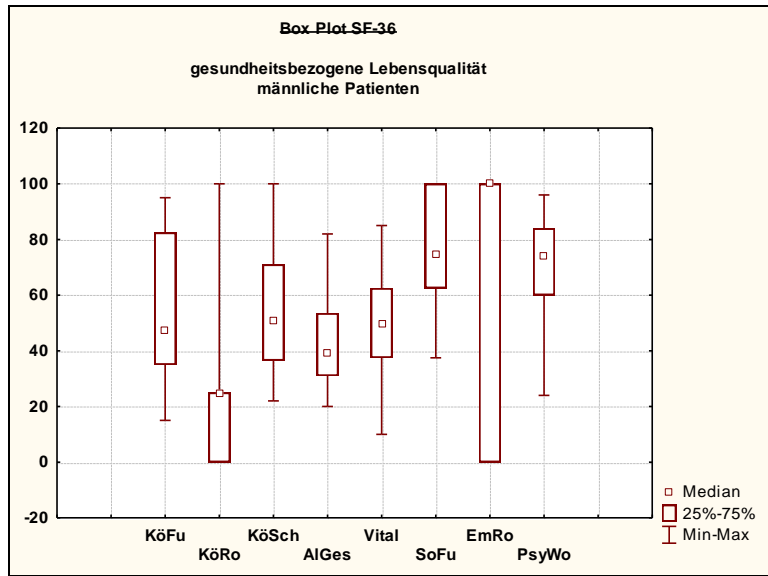


Abb. 7: Ergebnisse SF-36 (weibl. Patienten)

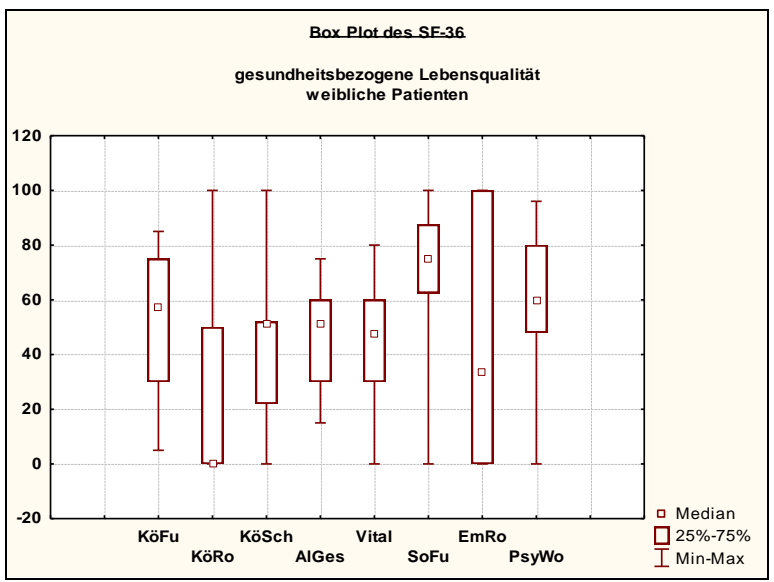

Abb. 8: Ergebnisse ESS (Patienten)

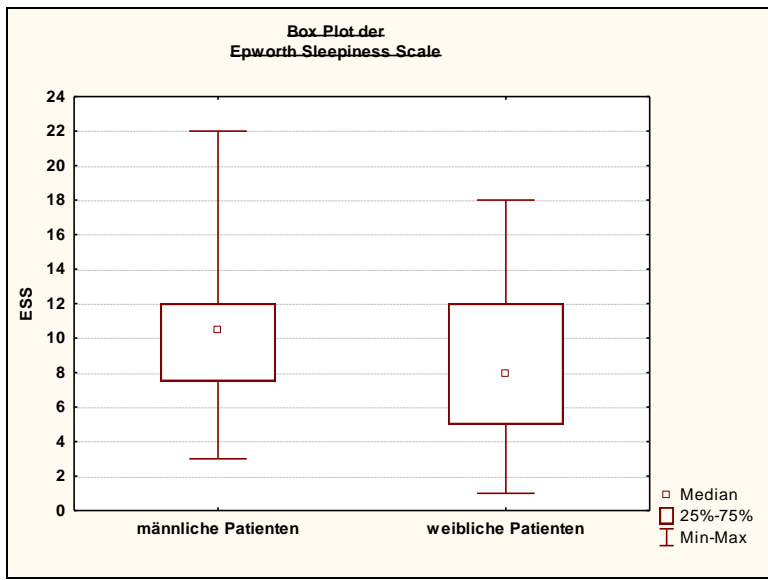

Abb. 9: Tabellarische Darstellung der Ergebnisse PSQI-Unterskalen (Patienten)

\begin{tabular}{|c|c|c|c|c|c|c|c|}
\hline & PSQI 1 & PSQI 2 & PSQI 3 & PSQI 4 & PSQI 5 & PSQI 6 & PSQI 7 \\
\hline \multicolumn{8}{|l|}{ männl. Pat. $(n=28)$} \\
\hline Minimum & 0 & 0 & 0 & 0 & 0 & 0 & 0 \\
\hline Maximum & 2 & 3 & 3 & 3 & 3 & 3 & 3 \\
\hline Mittelwert & 1,07 & 0,57 & 0,96 & 0,93 & 1,14 & 0,29 & 1,36 \\
\hline Standardabweichung & 0,66 & 0,74 & 1,10 & 1,15 & 0,59 & 0,85 & 0,91 \\
\hline \multicolumn{8}{|l|}{ weibl. Pat. $(n=22)$} \\
\hline Minimum & 0 & 0 & 0 & 0 & 0 & 0 & 0 \\
\hline Maximum & 3 & 3 & 3 & 3 & 2 & 3 & 3 \\
\hline Mittelwert & 1,18 & 1,00 & 1,36 & 1,32 & 1,32 & 0,27 & 1,14 \\
\hline Standardabweichung & 0,73 & 0,98 & 1,05 & 1,17 & 0,57 & 0,88 & 0,71 \\
\hline \multicolumn{8}{|l|}{ alle $(n=50)$} \\
\hline Minimum & 0 & 0 & 0 & 0 & 0 & 0 & 0 \\
\hline Maximum & 3 & 3 & 3 & 3 & 3 & 3 & 3 \\
\hline Mittelwert & 1,12 & 0,76 & 1,14 & 1,10 & 1,22 & 0,28 & 1,26 \\
\hline Standardabweichung & 0,69 & 0,87 & 1,09 & 1,16 & 0,58 & 0,86 & 0,83 \\
\hline
\end{tabular}

[PSQI 1 = subjektive Schlafqualität; PSQI 2 = Schlaflatenz; PSQI 3 = Schlafdauer; PSQI 4 = Schlafeffizienz; PSQI 5 = Schlafstörungen; PSQI 6 = Schlafmittelkonsum; PSQI 7 = Tagesschläfrigkeit $]$

Abb. 10: Ergebnisse PSQI (Patienten)

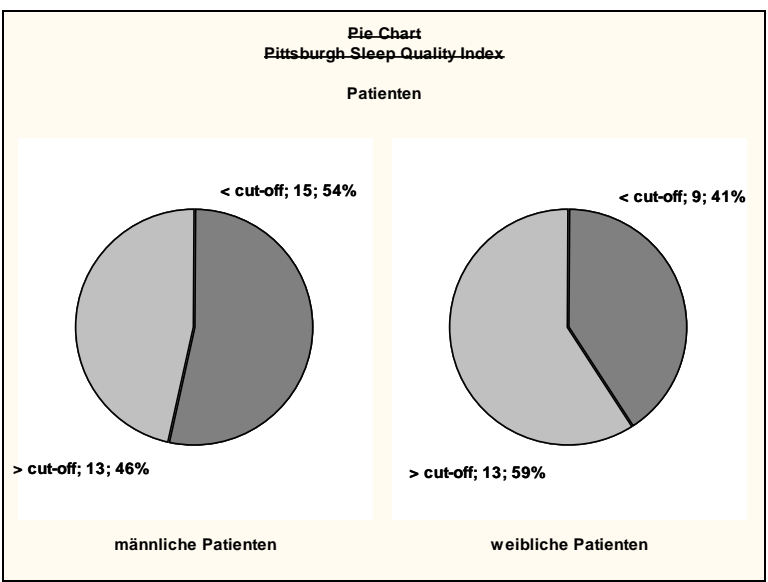


Abb. 11: Ergebnisse FAS (Betreuer)

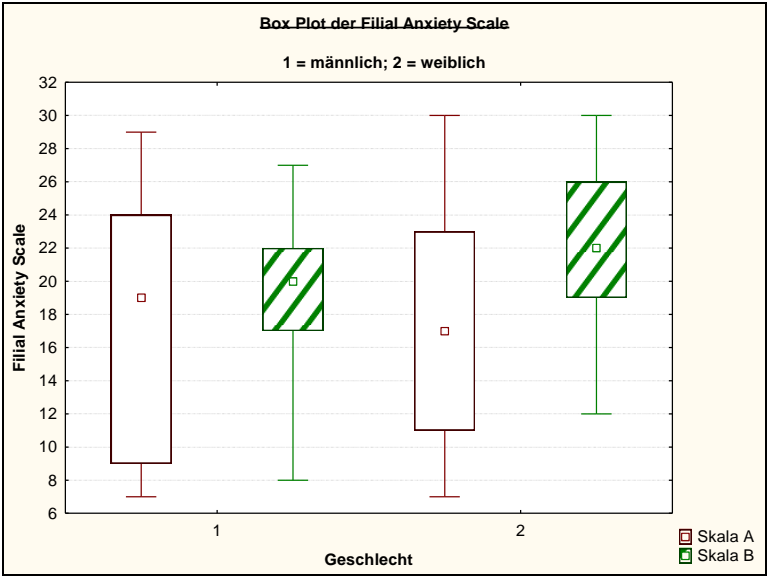

Abb. 13: Ergebnisse FAA nach Betreuerstatus

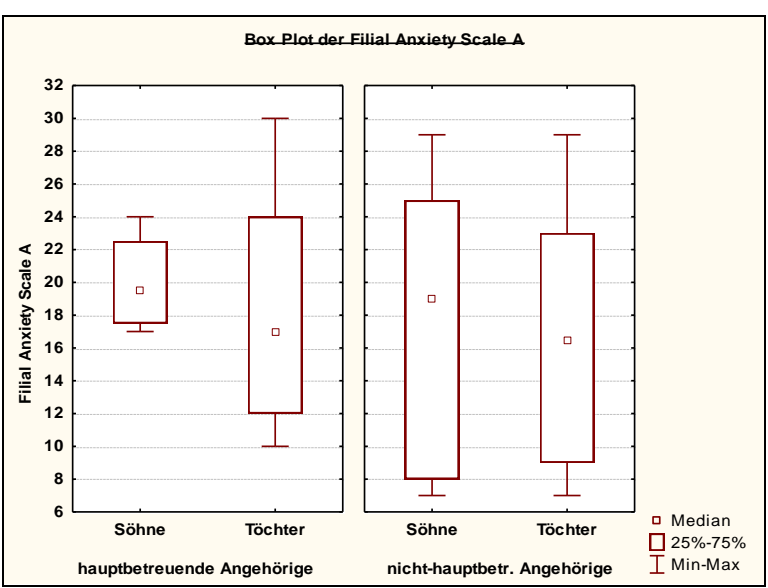

Abb. 15: Mittelwerte LFMS-A (Betreuer)

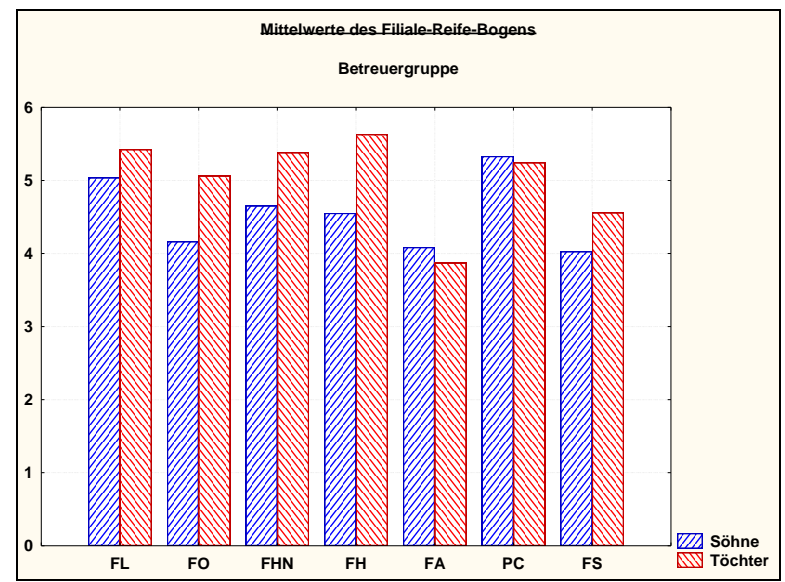

Abb. 12: Ergebnisse FAS nach Betreuerstatus

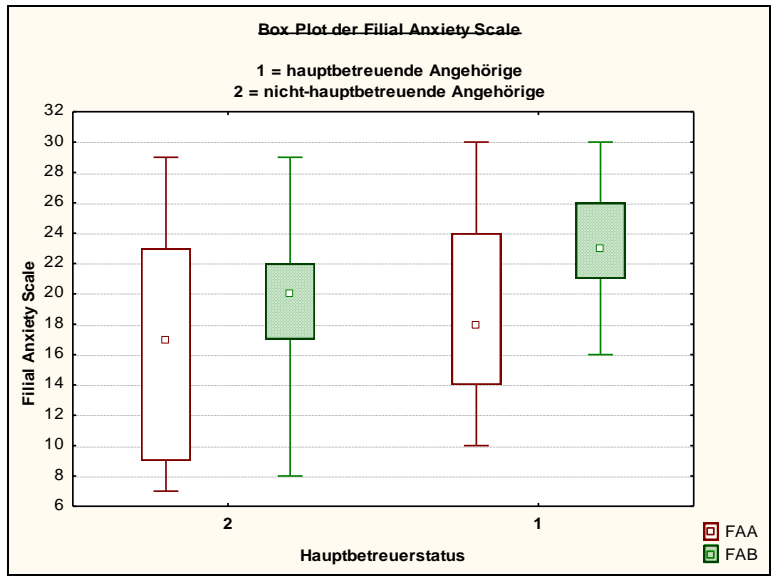

Abb. 14: Ergebnisse FAB nach Betreuerstatus

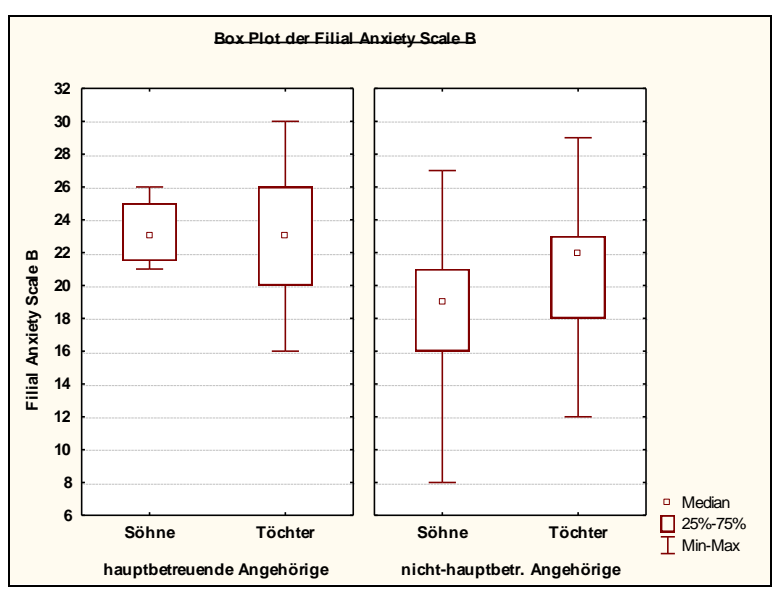

Abb. 16: Ergebnisse LFMS-A (betreuende Söhne)

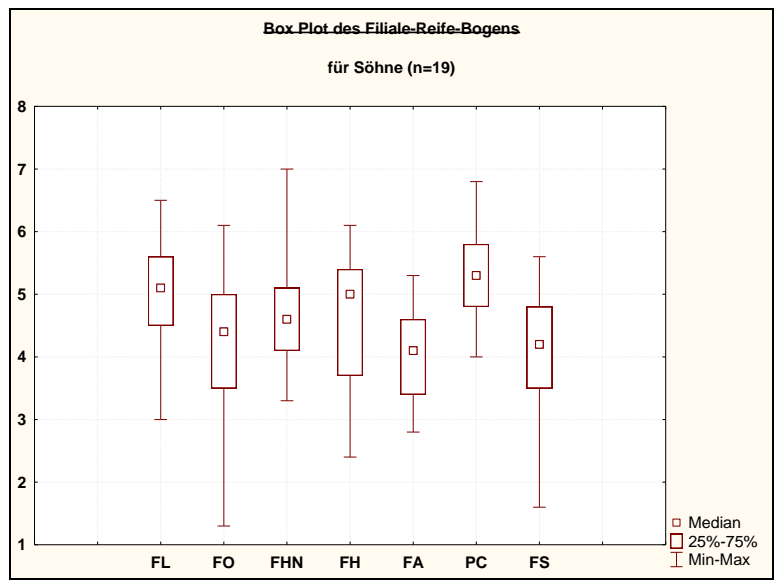


Abb. 17: Ergebnisse LFMS-A (betreuende Töchter)

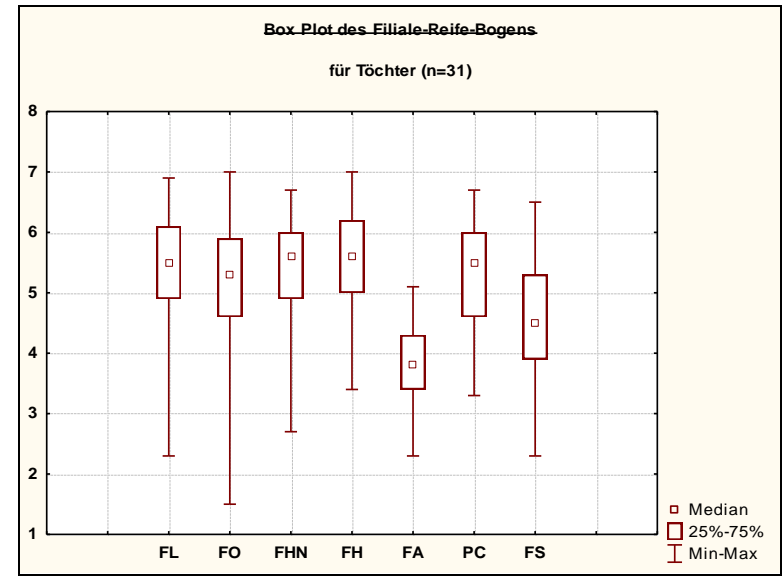

Abb. 19: Ergebnisse LFMS-A (Nicht-Hauptbetreuer)

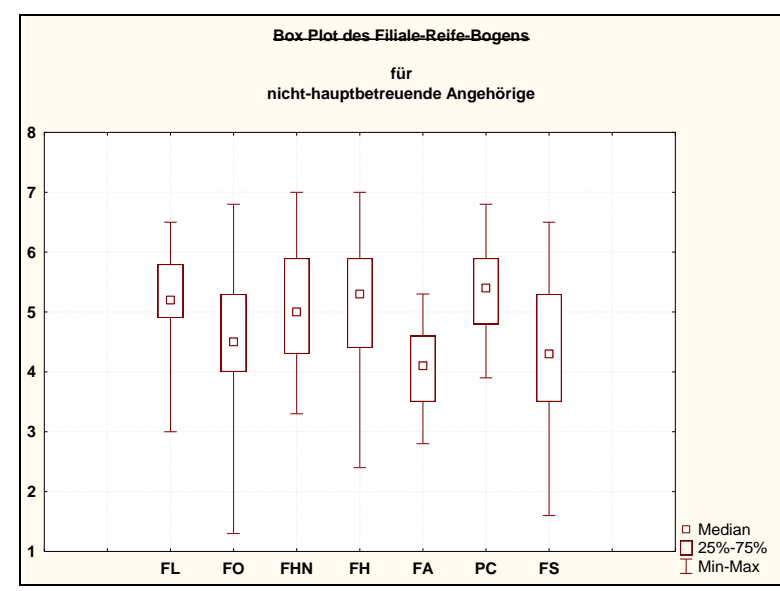

Abb. 21: Ergebnisse SF-36 (betreuende Söhne)

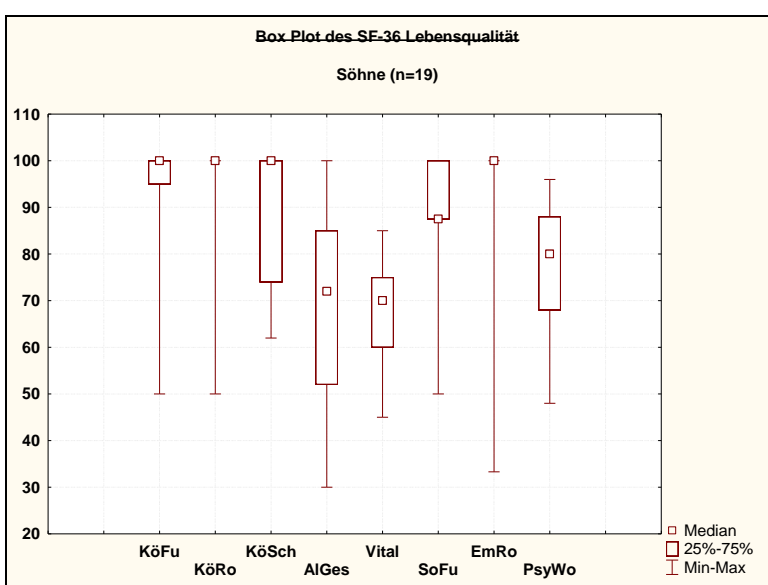

Abb. 18: Ergebnisse LFMS-A (Hauptbetreuer)

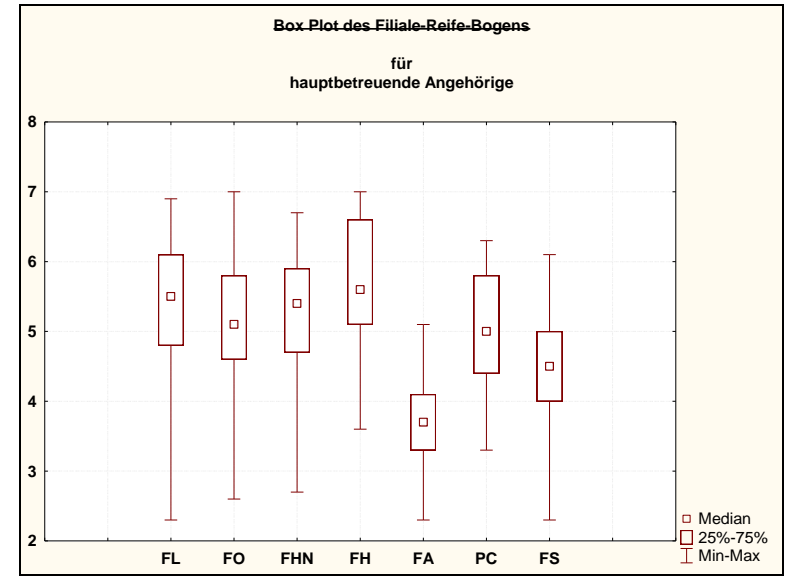

Abb. 20: Ergebnisse Belastungsbogen (Betreuer)

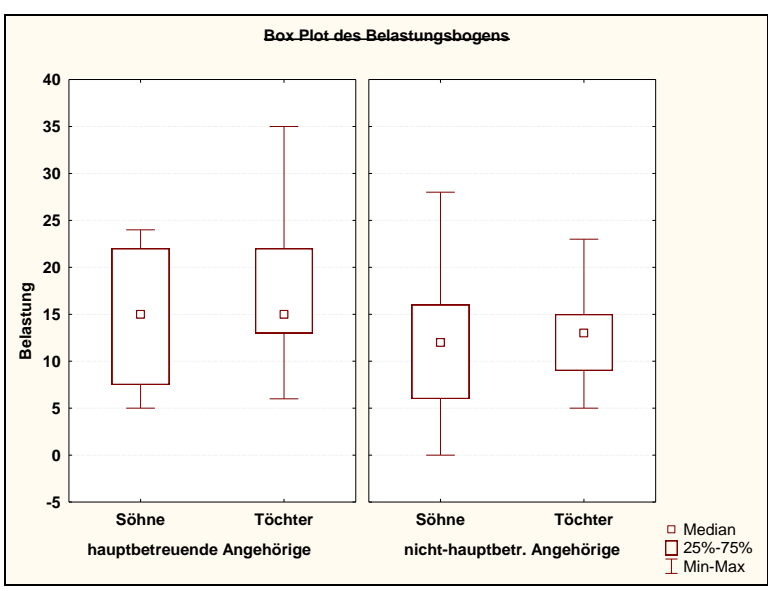

Abb. 22: Ergebnisse SF-36 (betreuende Töchter)

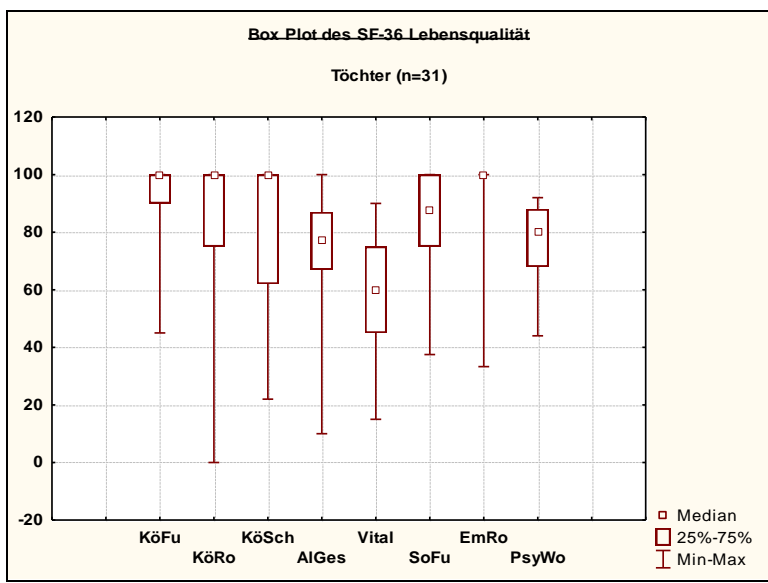


Abb. 23: Ergebnisse CESD-10 (Betreuer)

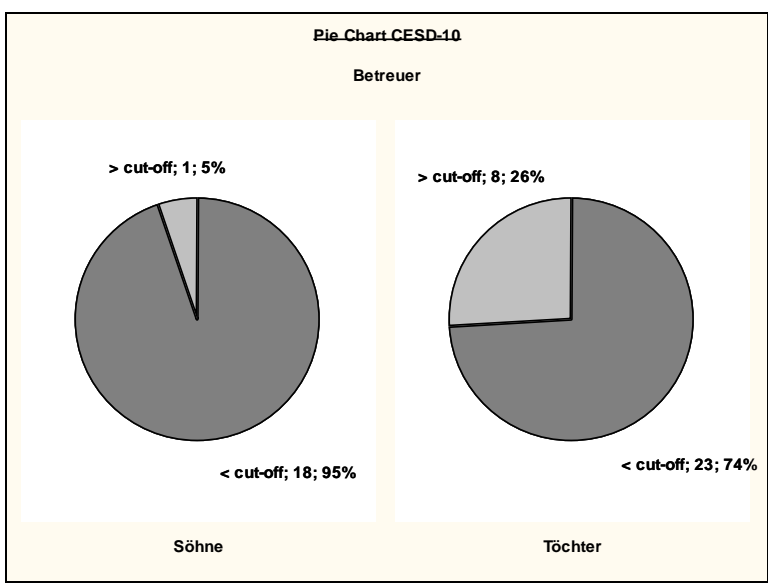

Abb. 24: Ergebnisse ESS (Betreuer)

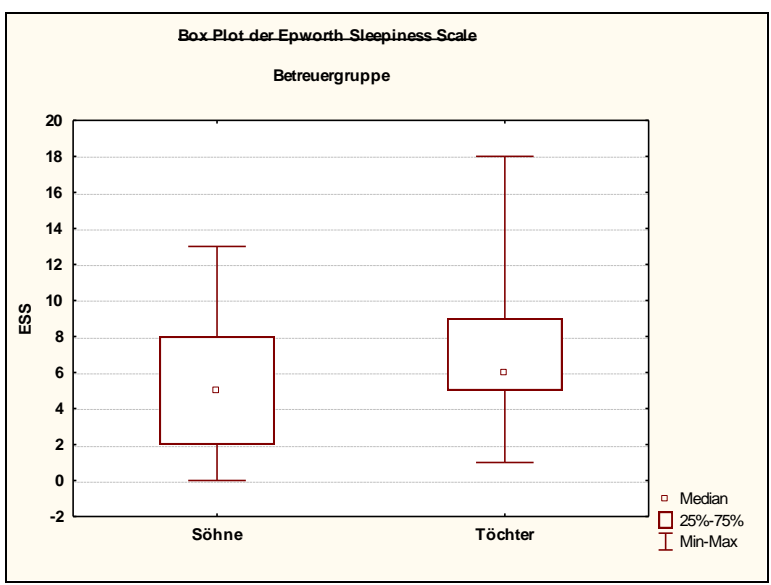

Abb. 25: Ergebnisse ESS nach Betreuerstatus

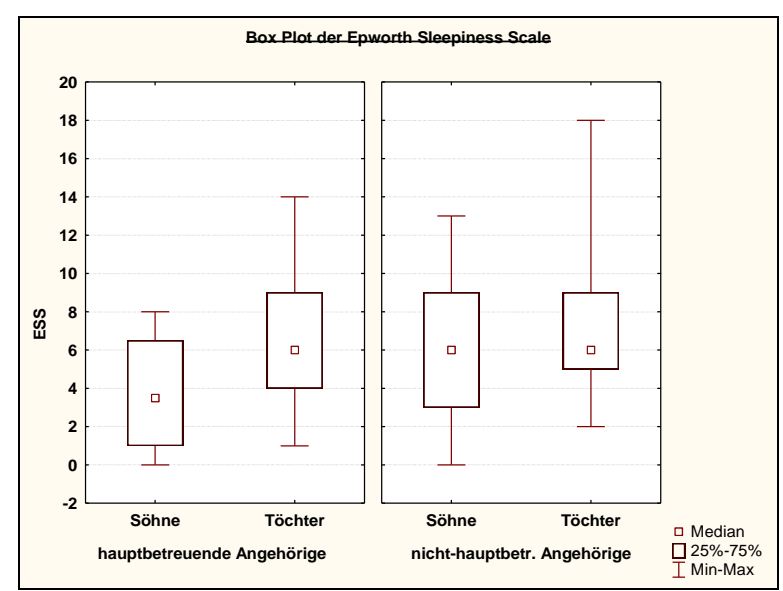

Abb. 26: Tabellarische Darstellung der Ergebnisse PSQI-Unterskalen (Betreuer)

\begin{tabular}{|l|r|r|r|r|r|r|r|}
\hline & PSQI 1 & PSQI 2 & PSQI 3 & PSQI 4 & PSQI 5 & PSQI 6 & PSQI 7 \\
\hline Söhne (n = 19) & & & & & & & \\
\hline Minimum & 0 & 0 & 0 & 0 & 0 & 0 & 0 \\
\hline Maximum & 2 & 3 & 2 & 2 & 1 & 0 & 2 \\
\hline Mittelwert & 0,95 & 0,89 & 0,63 & 0,37 & 0,89 & 0 & 0,68 \\
\hline Standardabweichung & 0,71 & 0,86 & 0,83 & 0,68 & 0,32 & 0 & 0,58 \\
\hline Töchter (n =31) & & & & & & & \\
\hline Minimum & 0 & 0 & 0 & 0 & 0 & 0 & 0 \\
\hline Maximum & 2 & 3 & 2 & 3 & 2 & 2 & 3 \\
\hline Mittelwert & 1,06 & 1,00 & 0,58 & 0,48 & 1,10 & 0,06 & 0,94 \\
\hline Standardabweichung & 0,63 & 0,89 & 0,72 & 0,77 & 0,54 & 0,36 & 0,77 \\
\hline alle (n = 50) & & & & & & & \\
\hline Minimum & 0 & 0 & 0 & 0 & 0 & 0 & 0 \\
\hline Maximum & 2 & 3 & 2 & 3 & 2 & 2 & 3 \\
\hline Mittelwert & 1,02 & 0,96 & 0,60 & 0,44 & 1,02 & 0,04 & 0,84 \\
\hline Standardabweichung & 0,65 & 0,88 & 0,76 & 0,73 & 0,47 & 0,28 & 0,71 \\
\hline
\end{tabular}

[PSQI 1 = subjektive Schlafqualität; PSQI 2 = Schlaflatenz; PSQI 3 = Schlafdauer; PSQI 4 = Schlafeffizienz; PSQI 5 = Schlafstörungen; PSQI 6 = Schlafmittelkonsum; PSQI 7 = Tagesschläfrigkeit $]$ 
Abb. 27: Ergebnisse PSQI (Betreuer)

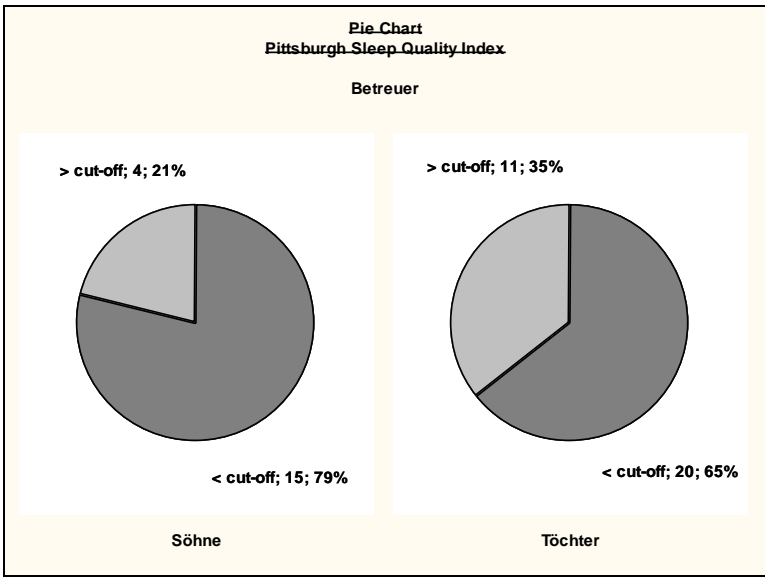

Abb. 29: Ergebnisse FAB (Betreuer/ Kontrollen)

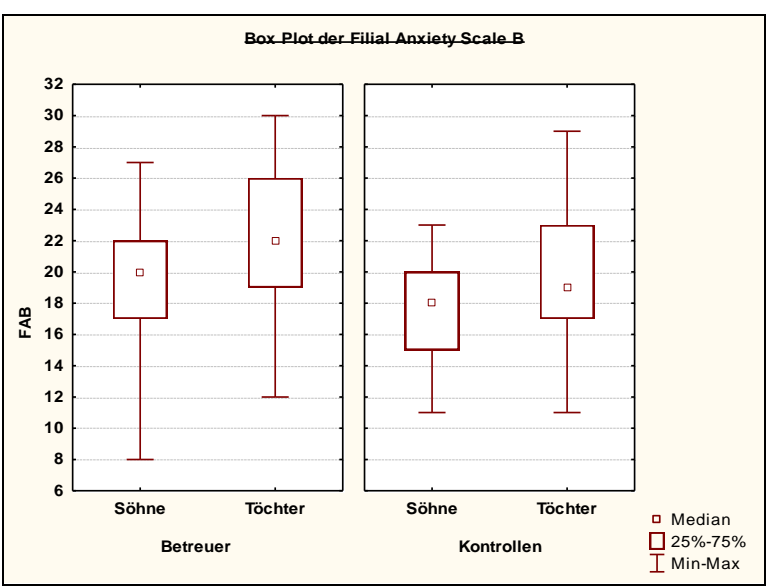

Abb. 31: Ergebnisse LFMS-A (weibl. Kontrollen)

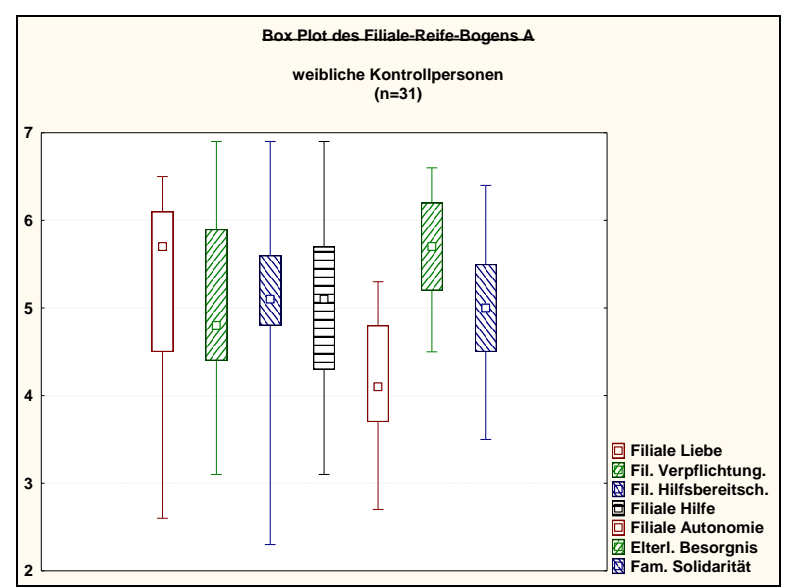

Abb. 28: Ergebnisse FAA (Betreuer/ Kontrollen)

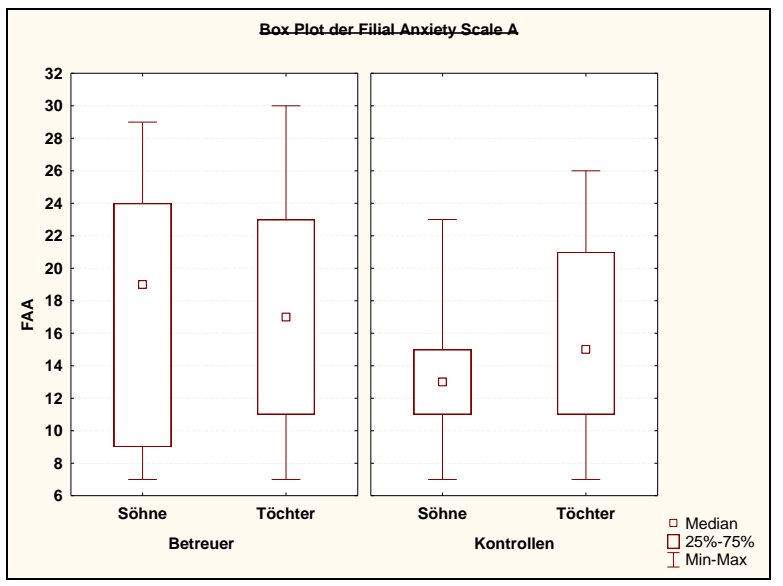

Abb. 30: Ergebnisse LFMS-A (männl. Kontrollen)

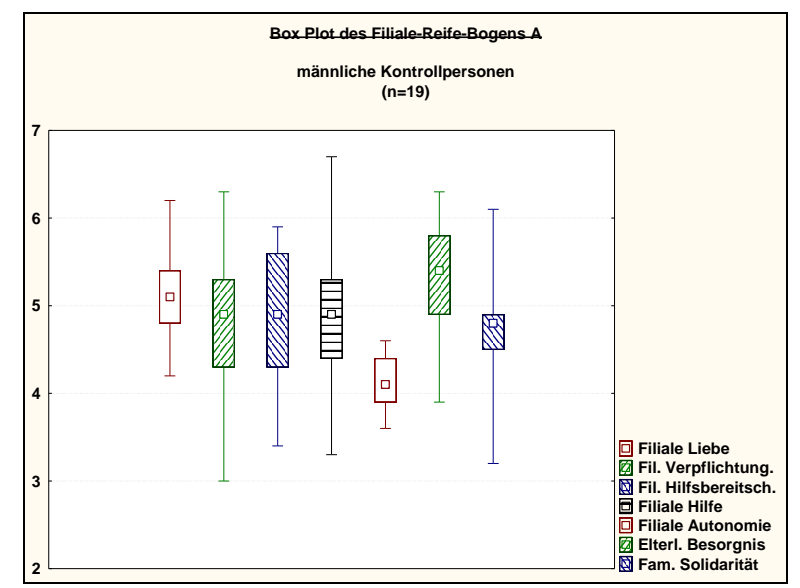

Abb. 32: Ergebnisse LFMS-A (männl. Betreuer/ Kontrollen)

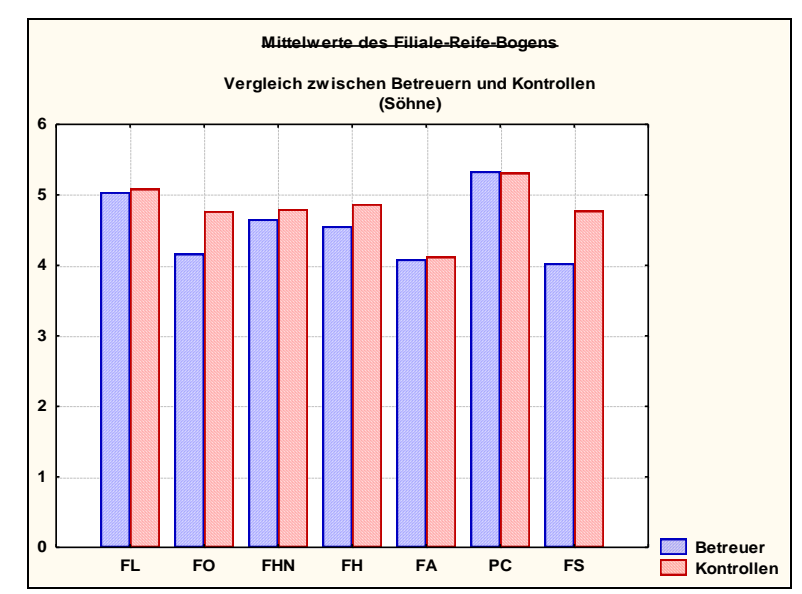


Abb. 33: Ergebnisse LFMS-A (weibl. Betreuer/ Kontrollen)

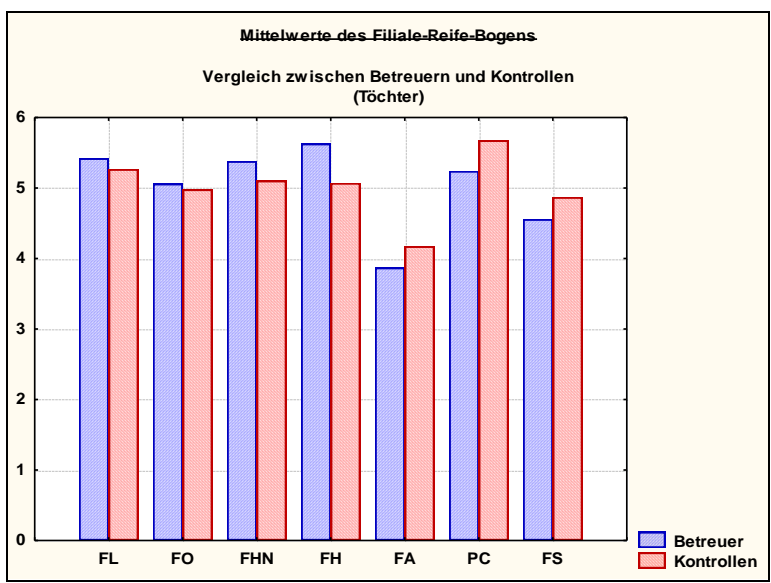

Abb. 35: Ergebnisse SF-36 (weibl. Kontrollen)

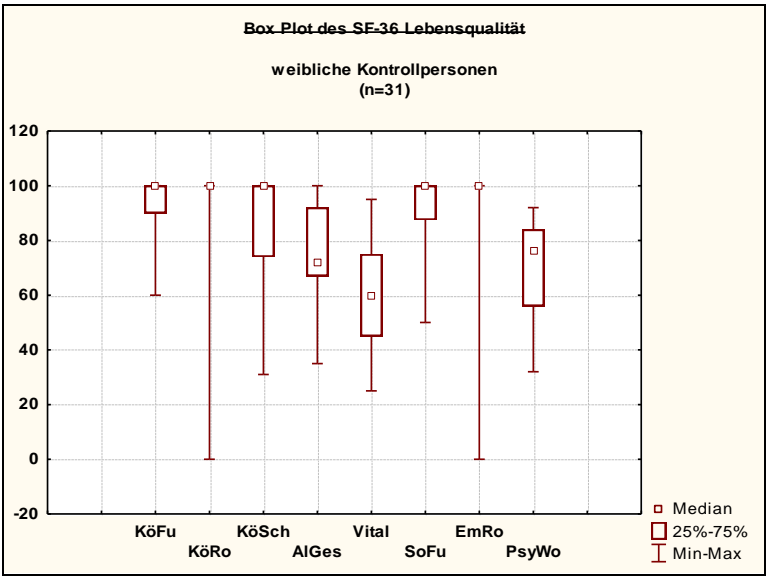

Abb. 37: Ergebnisse CESD-10 (Betreuer/ Kontrollen)

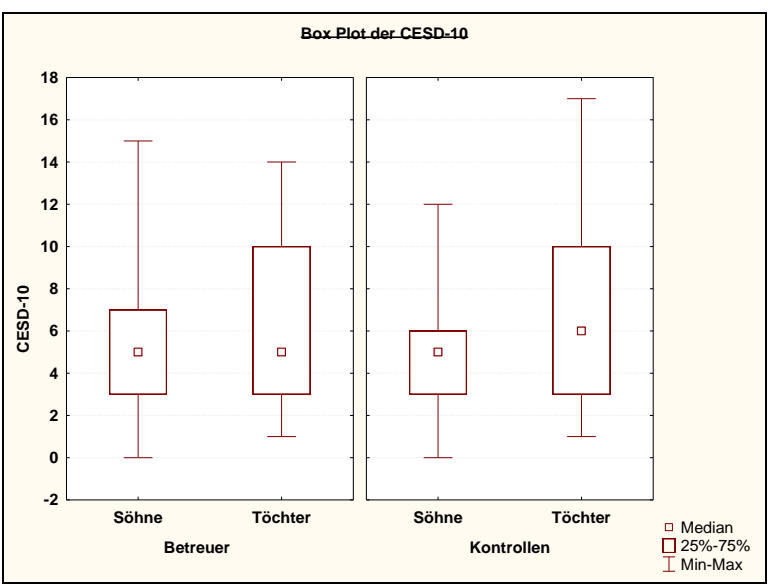

Abb. 34: Ergebnisse SF-36 (männl. Kontrollen)

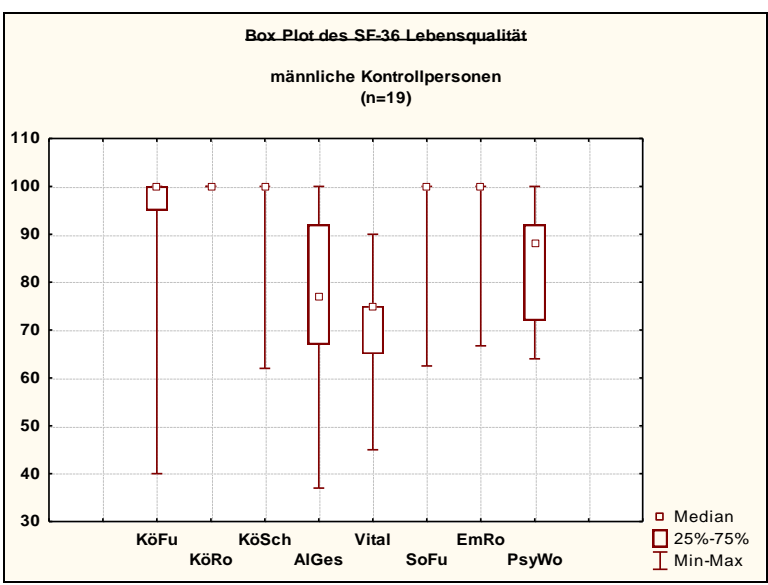

Abb. 36: Ergebnisse CESD-10 (Kontrollen)

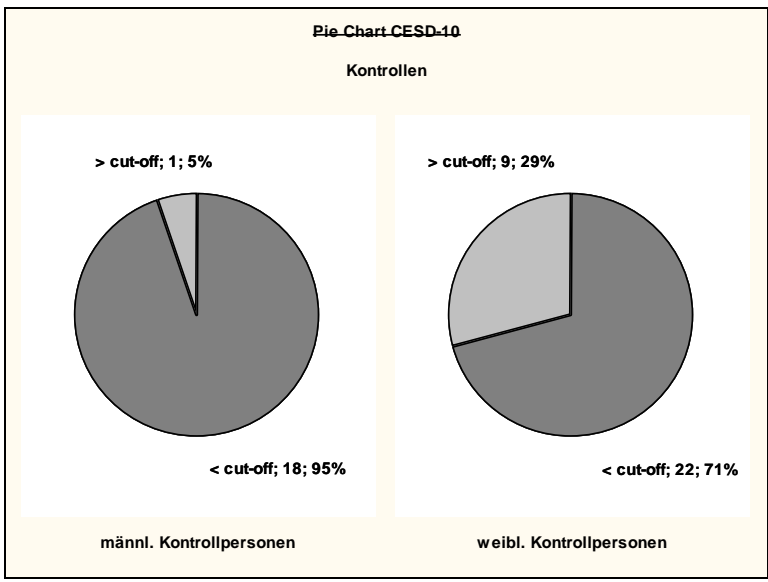

Abb. 38: Ergebnisse NOSGER (Betreuer/ Kontrollen)

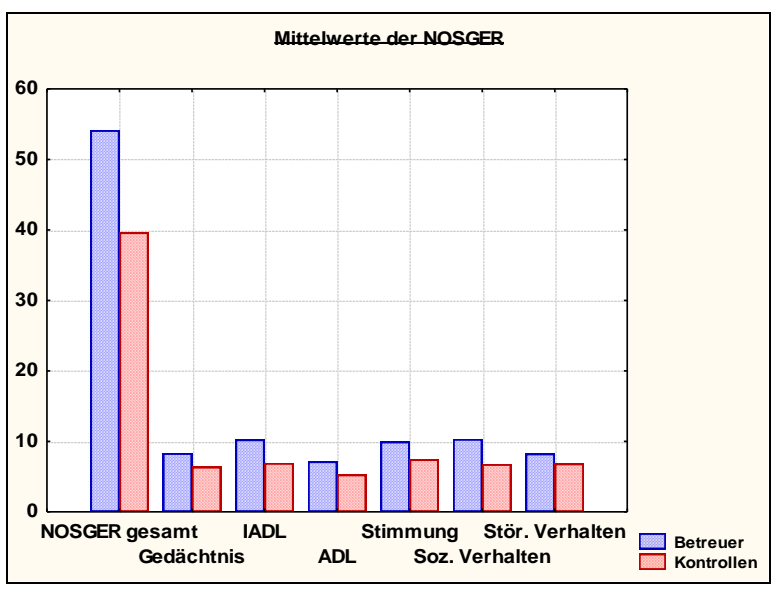


Abb. 39: Ergebnisse ESS (Betreuer/ Kontrollen)

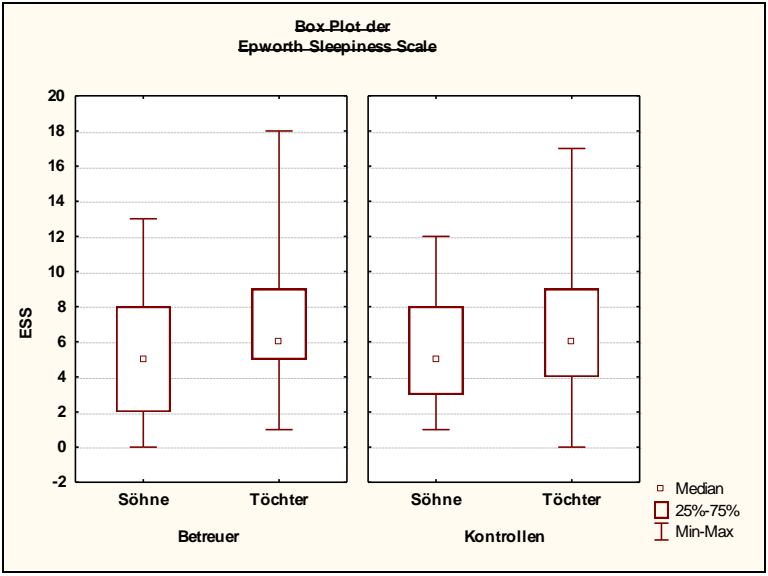

Abb. 41: Ergebnisse PSQI (Betreuer/ Kontrollen)

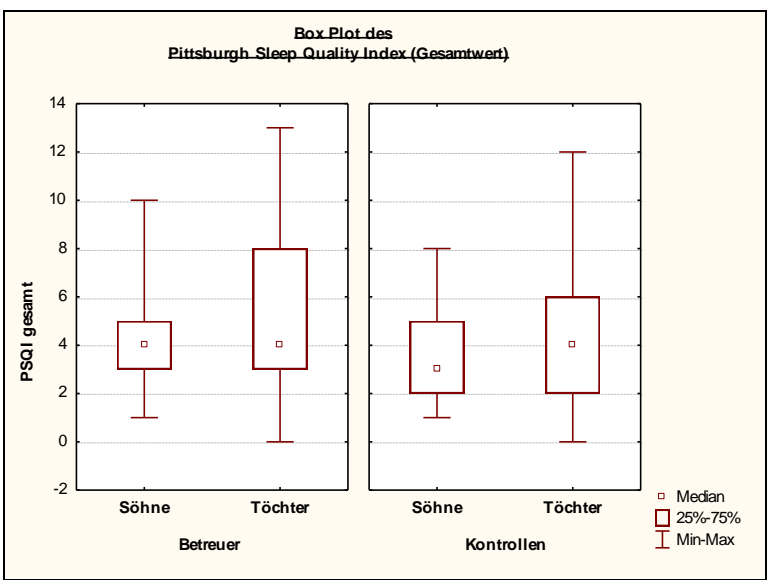

Abb. 43: Korrelation Belastungsbogen mit FO der LFMS-A (Betreuer)

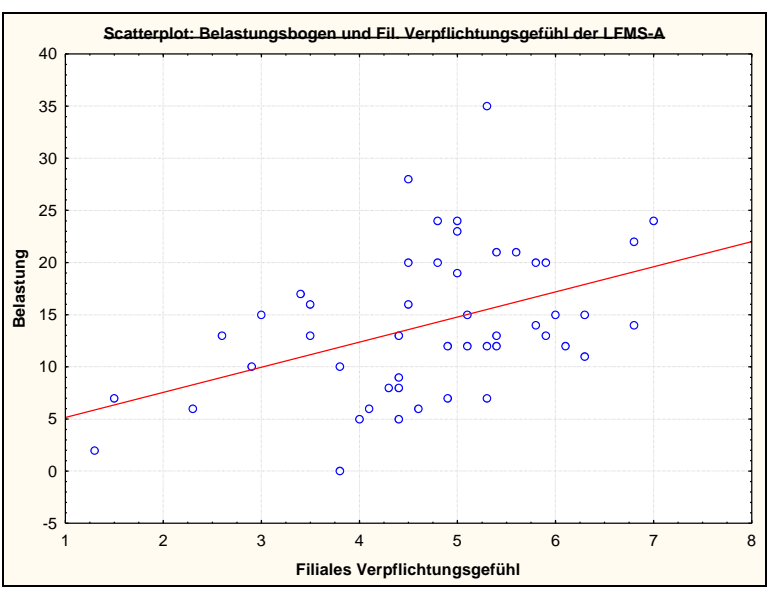

Abb. 40: Ergebnisse PSQI (Kontrollen)

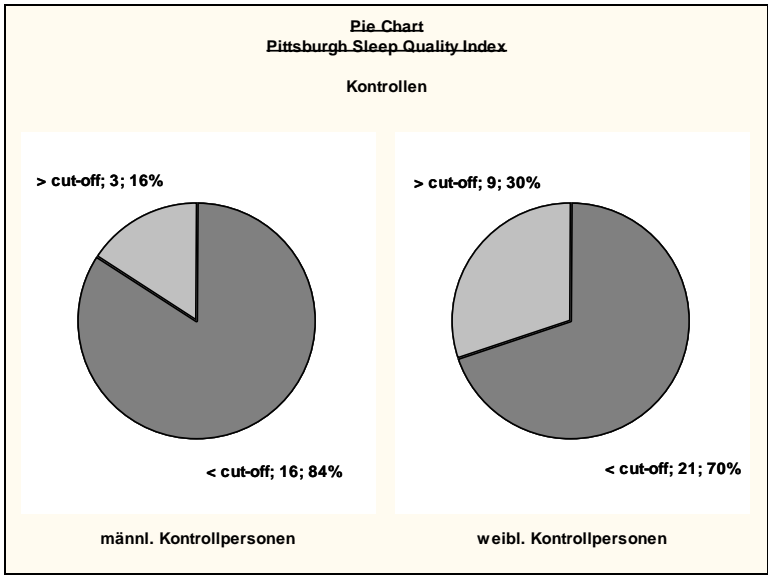

Abb. 42: Korrelation Belastungsbogen mit FAB (Betreuer)

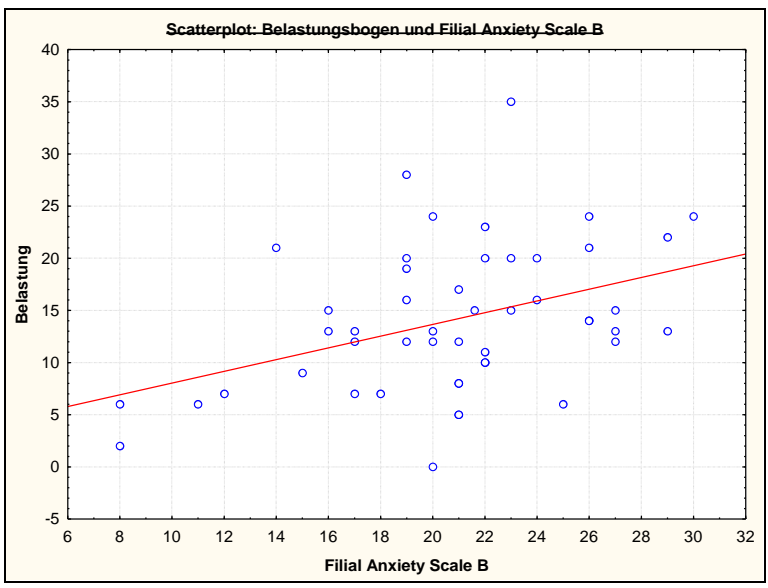


Abb. 44: Tabellarische Darstellung der Korrelationen LFMS-A und SF-36 (betreuende Söhne)

\begin{tabular}{|l|l|l|l|l|l|l|l|l|}
\hline \multicolumn{7}{|c|}{ Spearman's Rangkorrelation } \\
\hline & KöFu & KöRo & KöSch & AlGes & Vital & SoFu & EmRo & PsyWo \\
\hline FL & & & & & & & & \\
\hline FO & & & & & & & & \\
\hline FHN & & & & & & & & \\
\hline FH & & & & & & & & \\
\hline FA & & & & $* 0,54$ & & & & \\
\hline PC & & & & & & & & \\
\hline FS & & & & & & & & \\
\hline
\end{tabular}

Abb. 45: Tabellarische Darstellung der Korrelationen LFMS-A und SF-36 (betreuende Töchter)

\begin{tabular}{|c|c|c|c|c|c|c|c|c|}
\hline \multicolumn{9}{|c|}{$\begin{array}{l}\text { Spearman's Rangkorrelation } \\
{ }^{\circ} \mathrm{p}<0,1 * \mathrm{p}<0,05 * * \mathrm{p}<0,01 \quad * * * \mathrm{p}<0,001\end{array}$} \\
\hline & KöFu & KöRo & KöSch & AlGes & Vital & SoFu & EmRo & PsyWo \\
\hline FL & & & & & & & ${ }^{\circ} 0,34$ & $* 0,38$ \\
\hline FO & $*_{-0,44}$ & & & & & & & \\
\hline FHN & & & & & & & & ${ }^{\circ} 0,34$ \\
\hline FH & $* *-0,46$ & & & & & & & \\
\hline FA & ${ }^{\circ} 0,32$ & & & & & & & \\
\hline PC & & & & & & & & $* * 0,53$ \\
\hline FS & & & & & $* 0,44$ & $* 0,45$ & $* 0,43$ & ${ }^{\circ} 0,34$ \\
\hline
\end{tabular}

Abb. 46: Tabellarische Darstellung der Korrelationen PSQI-Unterskalen und SF-36 (Betreuer)

\begin{tabular}{|c|c|c|c|c|c|c|c|c|}
\hline \multicolumn{9}{|c|}{$\begin{array}{c}\text { Spearman's Rangkorrelation } \\
\text {. }\end{array}$} \\
\hline & KöFu & KöRo & KöSch & AlGes & Vital & SoFu & EmRo & PsyWo \\
\hline \multicolumn{9}{|l|}{ alle } \\
\hline PSQI g & $* * *-0,49$ & $* *_{-} 0,43$ & & $* *_{-} 0,37$ & $* *_{-} 0,42$ & & & $*_{-0,29}$ \\
\hline PSQI 1 & $*_{-0,35}$ & $* *_{-}-0,43$ & & & $*_{-0,35}$ & & $*_{-} 0,30$ & $* *_{-} 0,40$ \\
\hline PSQI 2 & $* *_{-}-0,40$ & ${ }^{\circ}-0,26$ & ${ }^{\circ}-0,27$ & $*_{-0,36}$ & $* *_{-} 0,42$ & & & $* *_{-}-0,38$ \\
\hline PSQI 3 & & ${ }^{\circ}-0,26$ & & & & & & \\
\hline PSQI 4 & $* * *-0,50$ & $* *_{-}-0,40$ & & & $*_{-} 0,35$ & & & \\
\hline PSQI 5 & $*_{-} 0,35$ & $* *_{-}-0,40$ & & & & & & \\
\hline \multicolumn{9}{|l|}{ PSQI 6} \\
\hline PSQI 7 & $*_{-0,34}$ & ${ }^{\circ}-0,27$ & & ${ }^{\circ}-0,27$ & $* * *-0,49$ & & & $*_{-} 0,32$ \\
\hline
\end{tabular}

[PSQI g = Gesamtwert PSQI; PSQI 1 = subjektive Schlafqualität; PSQI 2 = Schlaflatenz; PSQI 3 =

Schlafdauer; PSQI 4 = Schlafeffizienz; PSQI 5 = Schlafstörungen; PSQI 6 = Schlafmittelkonsum; PSQI $7=$ Tagesschläfrigkeit] 
Abb. 47: Tabellarische Darstellung der Korrelationen PSQI-Unterskalen und SF-36 (betreuende Söhne)

\begin{tabular}{|l|c|c|c|c|c|c|c|c|}
\hline \multicolumn{7}{|c|}{ Spearman's Rangkorrelation } \\
\hline & KöFu & KöRo & KöSch & AlGes & Vital & SoFu & EmRo & PsyWo \\
\hline Söhne & & & & & & & & \\
\hline PSQI g & & & & ${ }^{*}-0,52$ & & & & \\
\hline PSQI 1 & & & & & & & & \\
\hline PSQI 2 & & & ${ }^{*}-0,47$ & $*_{-}-0,53$ & ${ }^{\circ}-0,44$ & & & \\
\hline PSQI 3 & & & & & & & & $*+0,52$ \\
\hline PSQI 4 & & & & & & & & \\
\hline PSQI 5 & & & ${ }^{\circ} 0,42$ & & & & & \\
\hline PSQI 6 & & & & & & & & \\
\hline PSQI 7 & & & & & & & & \\
\hline
\end{tabular}

[PSQI g = Gesamtwert PSQI; PSQI 1 = subjektive Schlafqualität; PSQI 2 = Schlaflatenz; PSQI 3 = Schlafdauer; PSQI 4 = Schlafeffizienz; PSQI 5 = Schlafstörungen; PSQI 6 = Schlafmittelkonsum; PSQI 7 =

Tagesschläfrigkeit]

Abb. 48: Tabellarische Darstellung der Korrelationen PSQI-Unterskalen und SF-36 (betreuende Töchter)

\begin{tabular}{|c|c|c|c|c|c|c|c|c|}
\hline \multicolumn{9}{|c|}{ Spearman's Rangkorrelation } \\
\hline & KöFu & KöRo & KöSch & AlGes & Vital & SoFu & EmRo & PsyWo \\
\hline Töchter & & & & & & & & \\
\hline PSQI g & $* * *-0,68$ & $* * *-0,57$ & & & $* *_{-} 0,48$ & & & $*_{-0,42}$ \\
\hline PSQI 1 & $* * *-0,60$ & $* *-0,52$ & $*_{-0,40}$ & & $*_{-}-0,40$ & & $*_{-} 0,37$ & $*_{-0,41}$ \\
\hline PSQI 2 & $* * *_{-} 0,57$ & & & & $*_{-0,41}$ & & & $*_{-} 0,43$ \\
\hline PSQI 3 & & $* *-0,50$ & & & & & & \\
\hline PSQI 4 & $* * *-0,74$ & $* * *-0,62$ & $*_{-0,45}$ & & ${ }^{\circ}-0,35$ & & & \\
\hline PSQI 5 & $* *-0,53$ & $*_{-0,44}$ & $*_{-}-0,45$ & & & & & \\
\hline PSQI 6 & & & & & & & & \\
\hline PSQI 7 & $*_{-} 0,41$ & $*_{-0,36}$ & & $* *_{-} 0,49$ & $* * *-0,60$ & ${ }^{\circ}-0,35$ & & ${ }^{\circ}-0,34$ \\
\hline
\end{tabular}

[PSQI g = Gesamtwert PSQI; PSQI 1 = subjektive Schlafqualität; PSQI 2 = Schlaflatenz; PSQI 3 = Schlafdauer; PSQI 4 = Schlafeffizienz; PSQI 5 = Schlafstörungen; PSQI 6 = Schlafmittelkonsum; PSQI 7 =

Tagesschläfrigkeit]

Abb. 49: Korrelationen KöFu/ KöRo und PSQI (Betreuer)

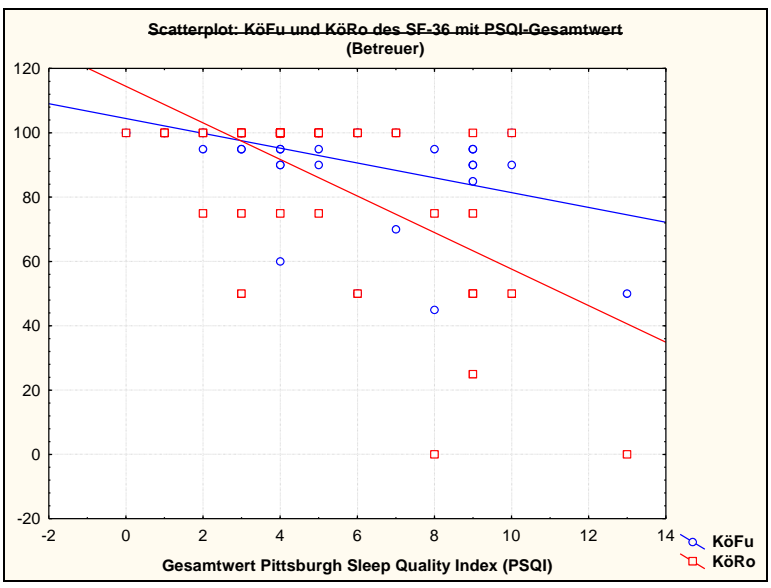

Abb. 50: Korrelationen KöSch/ AlGes und PSQI (Betreuer)

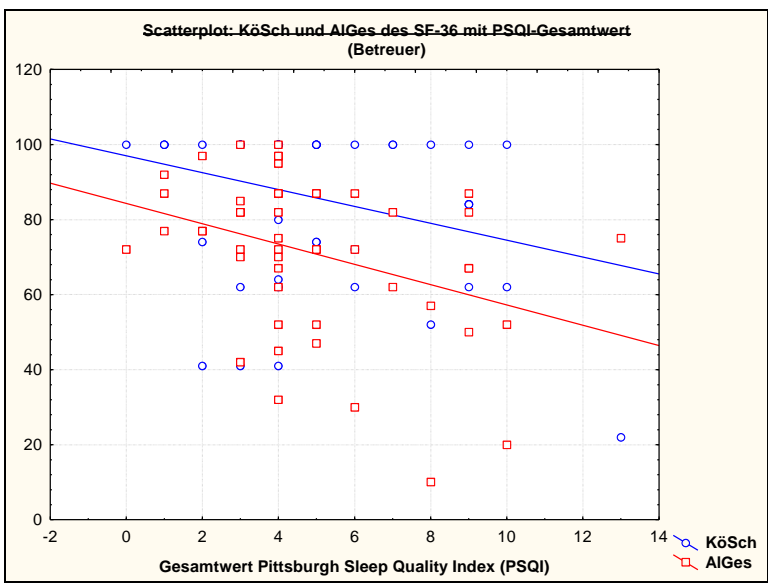


Abb. 51: Korrelationen Vital/ SoFu und PSQI (Betreuer)

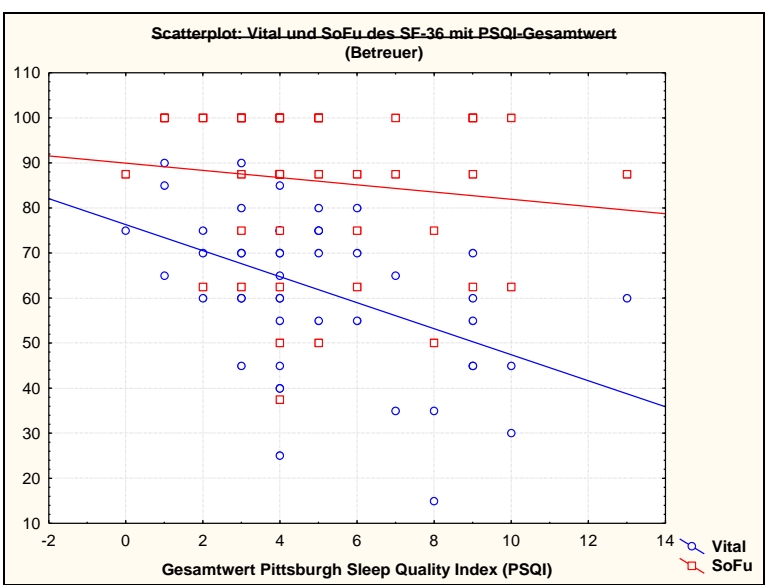

Abb. 53: Korrelation KöFu und ESS (Patienten)

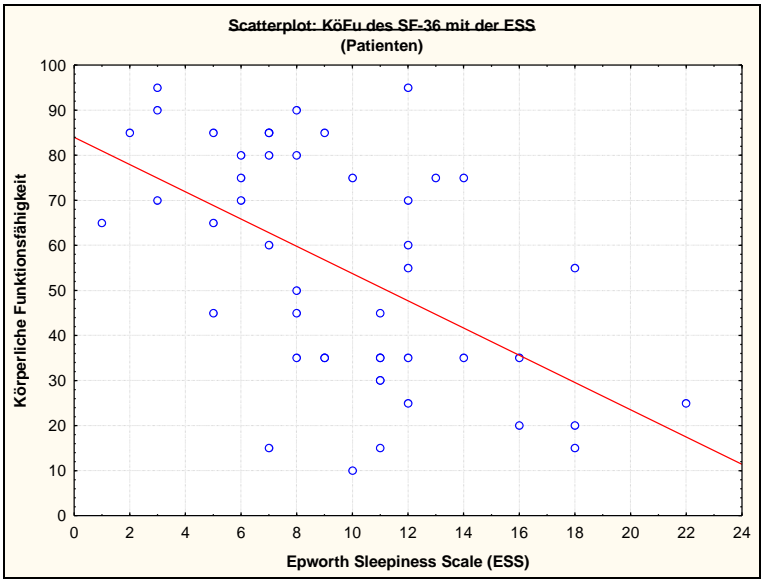

Abb. 55: Korrelationen AlGes/ EmRo und ESS (männl. Patienten)

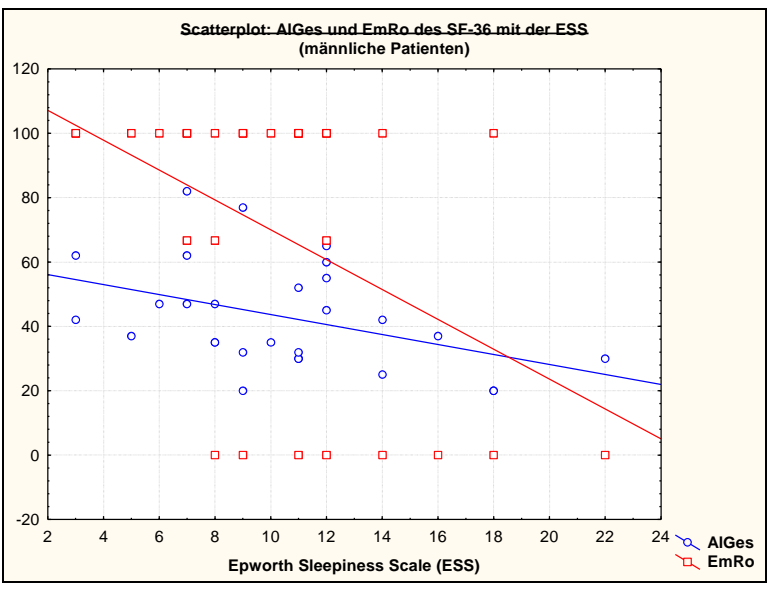

Abb. 52: Korrelationen EmRo/ PsyWo und PSQI (Betreuer)

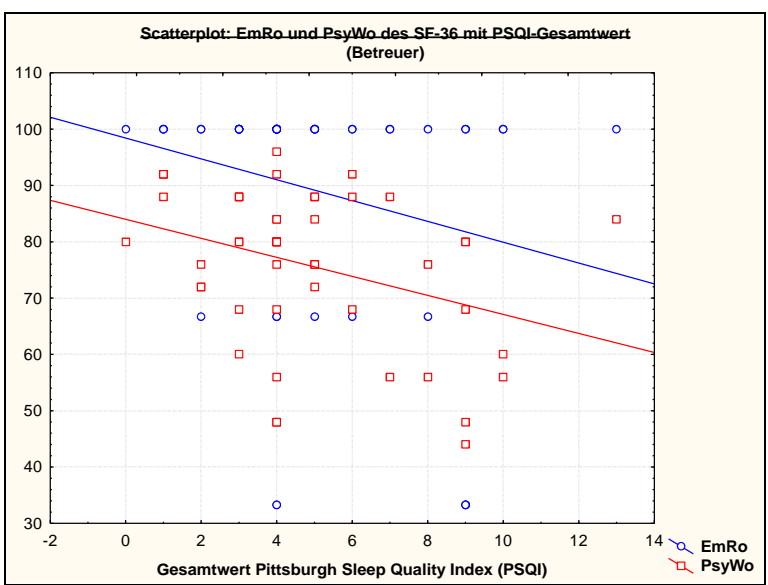

Abb. 54: Korrelationen KöFu/ KöRo und ESS (männl. Patienten)

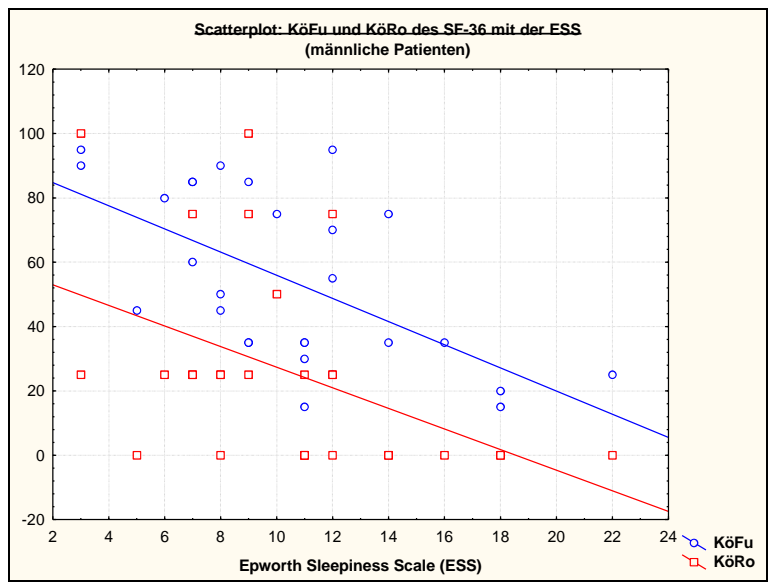

Abb. 56: Korrelationen KöSch/ AlGes mit PSQI (Patienten)

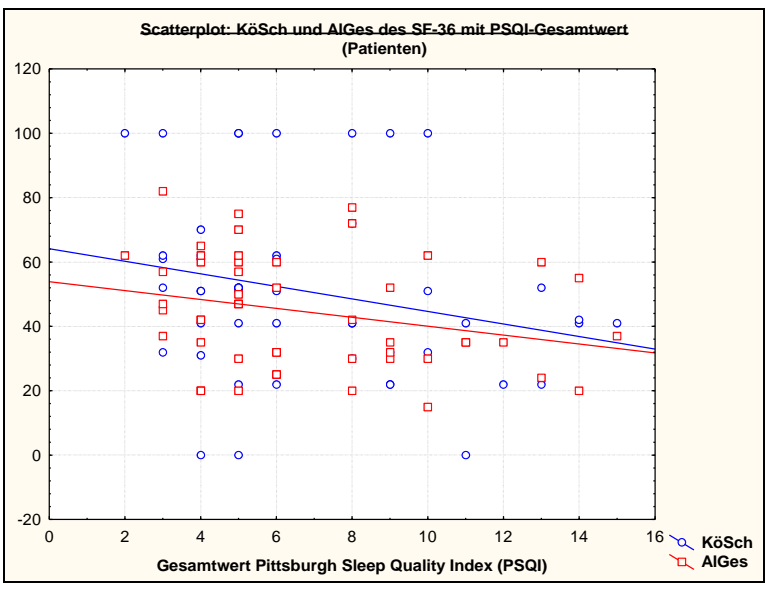


Abb. 57: Korrelationen KöFu/ KöRo und PDSS (Patienten)

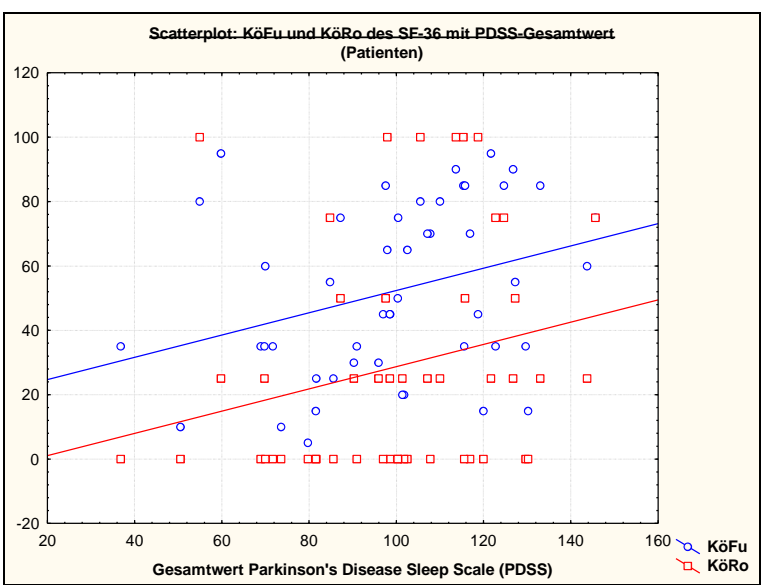

Abb. 59: Korrelationen Vital/ SoFu und PDSS (Patienten)

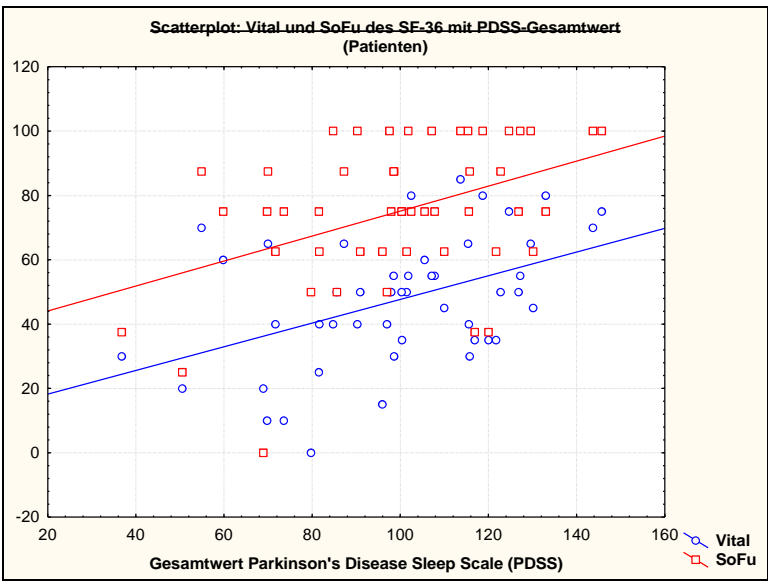

Abb. 61: Korrelation Belastungsbogen und NOSGER (Betreuer)

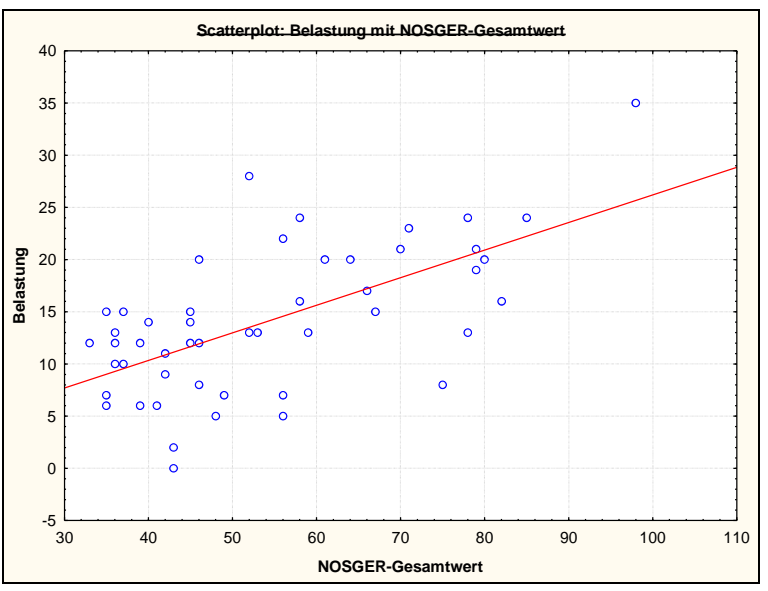

Abb. 58: Korrelationen KöSch/ AlGes und PDSS (Patienten)

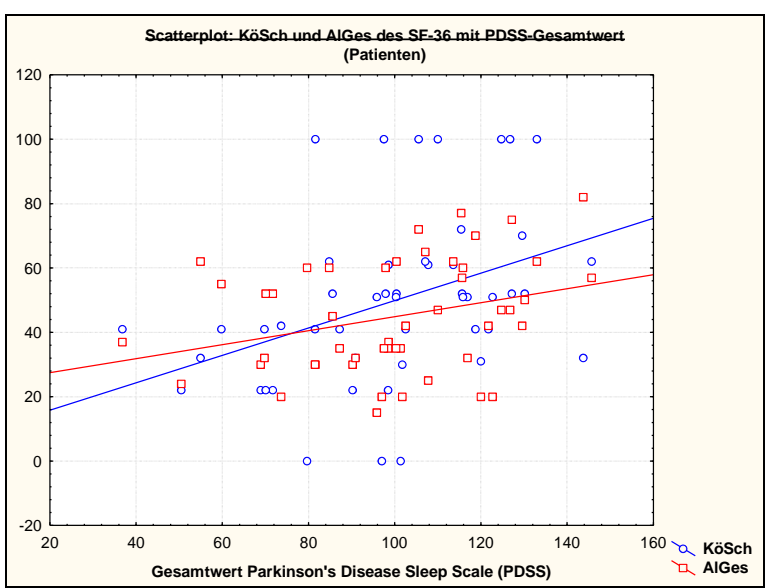

Abb. 60: Korrelationen EmRo und PsyWo und PDSS (Patienten)

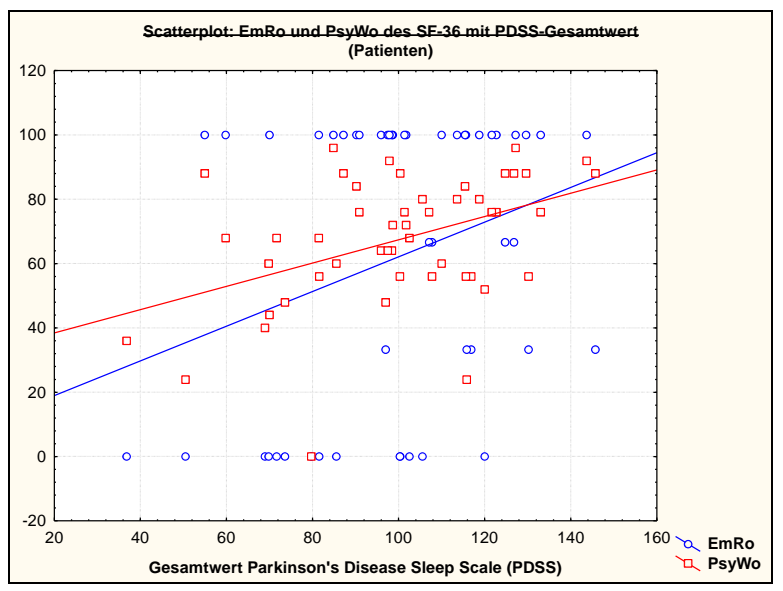

Abb. 62: Korrelation SoFu und NOSGER (Betreuer)

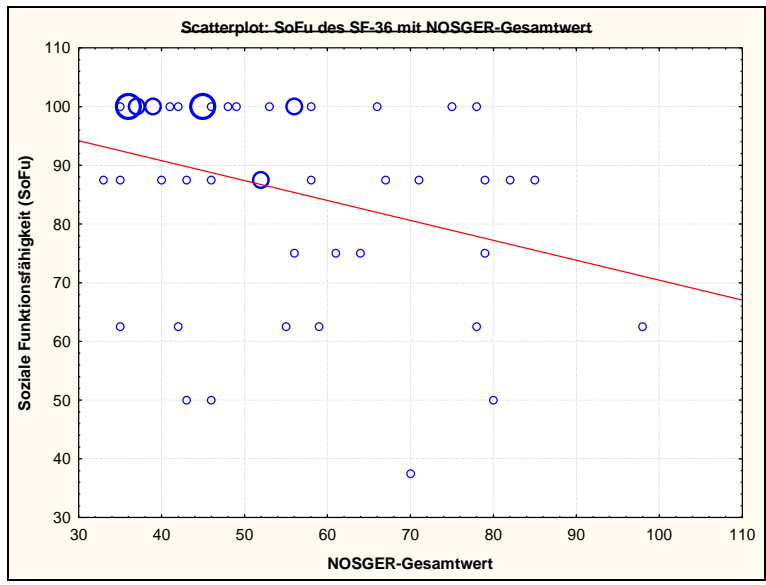


Abb. 64: Korrelationen KöFu/ KöRo (Patienten) und Belastung (Betreuer)

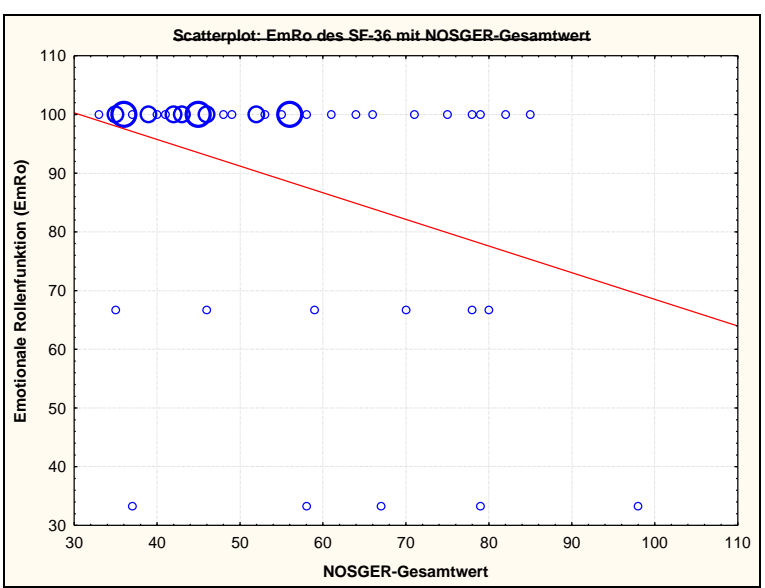

Abb. 65: Korrelationen KöSch/ AlGes (Patienten) und Belastung (Betreuer)

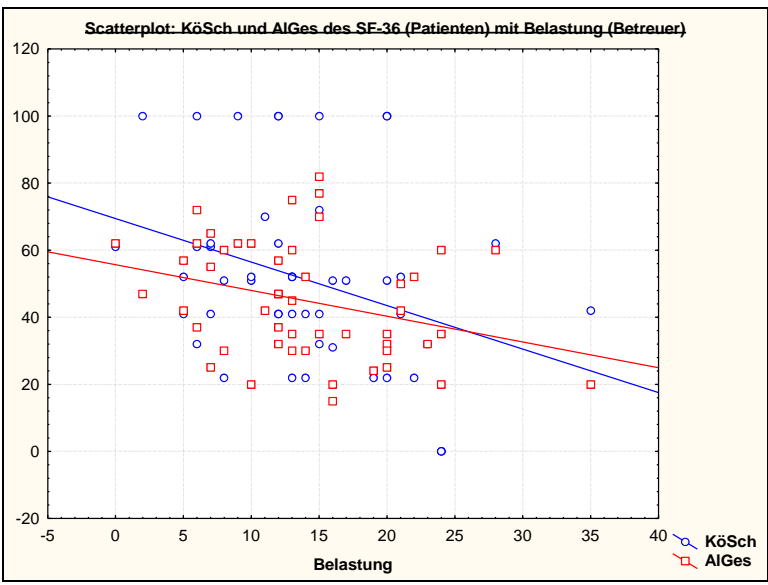

Abb. 67: Korrelation GDS (Patienten) und Belastung (Betreuer)

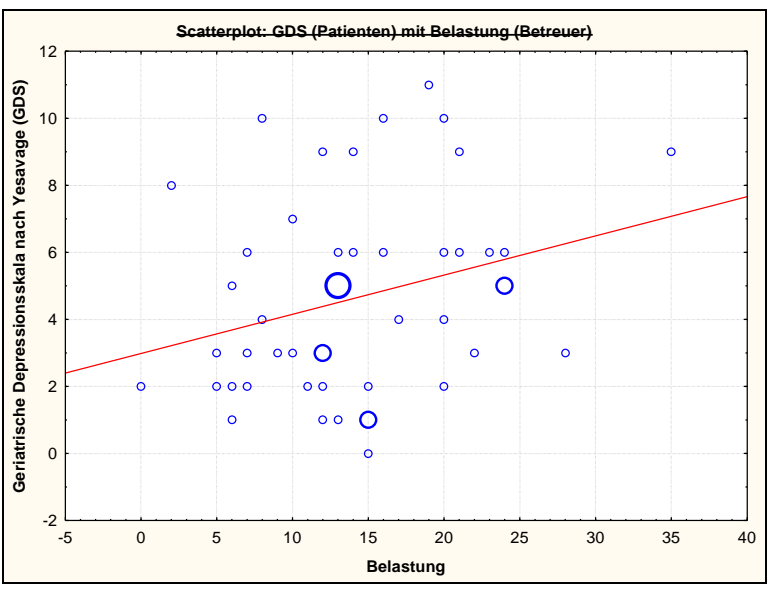

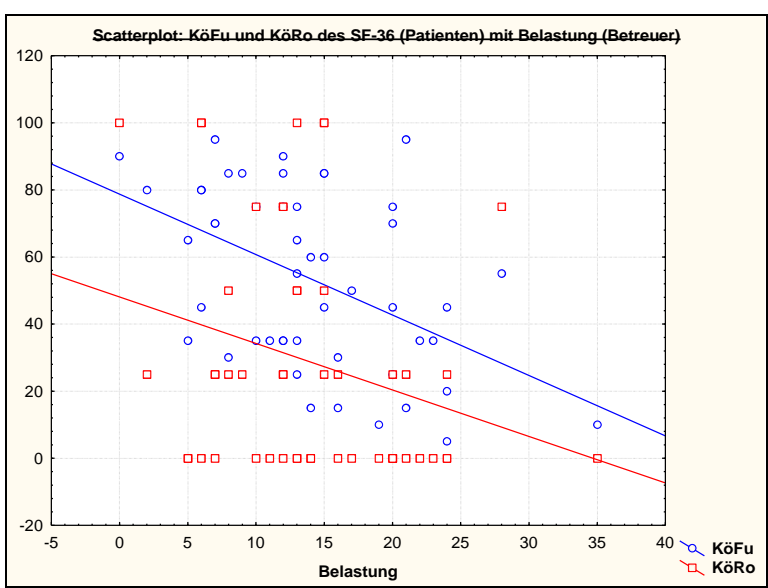

Abb. 66: Korrelationen Vital/ SoFu (Patienten) und Belastung (Betreuer)

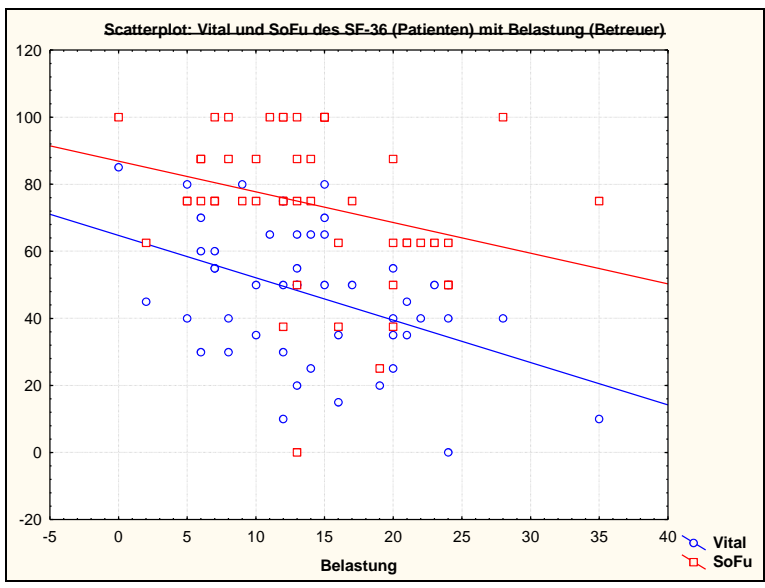

Abb. 68: Korrelation CESD-10 (Patienten) und Belastung (Betreuer)

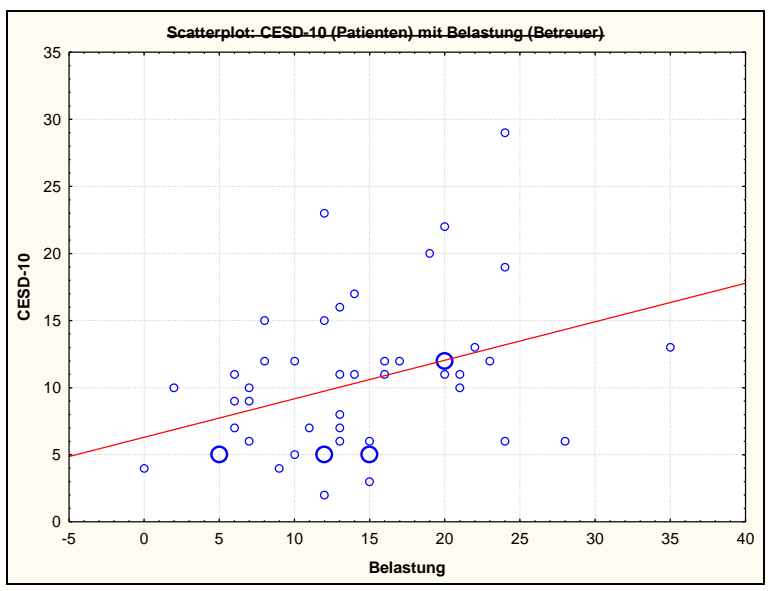


Abb. 69: Korrelation Belastung (Betreuer) und UPDRS

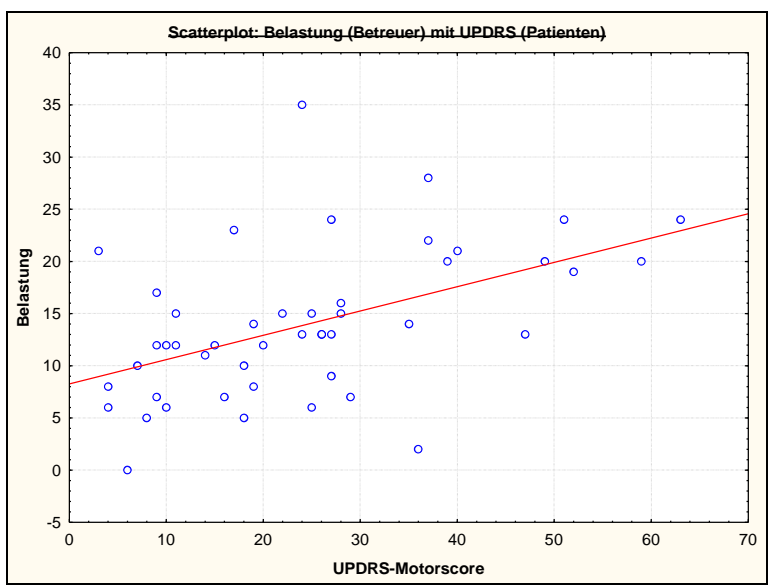

Abb. 71: Korrelation FAA und PSQI-Unterscore 5 (Betreuer)

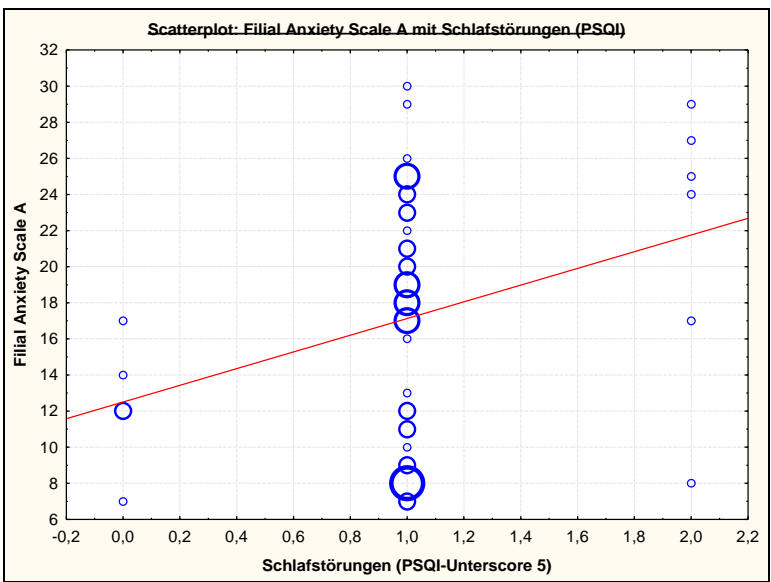

Abb. 70: Korrelation Belastung (Betreuer) und H\&YStadium

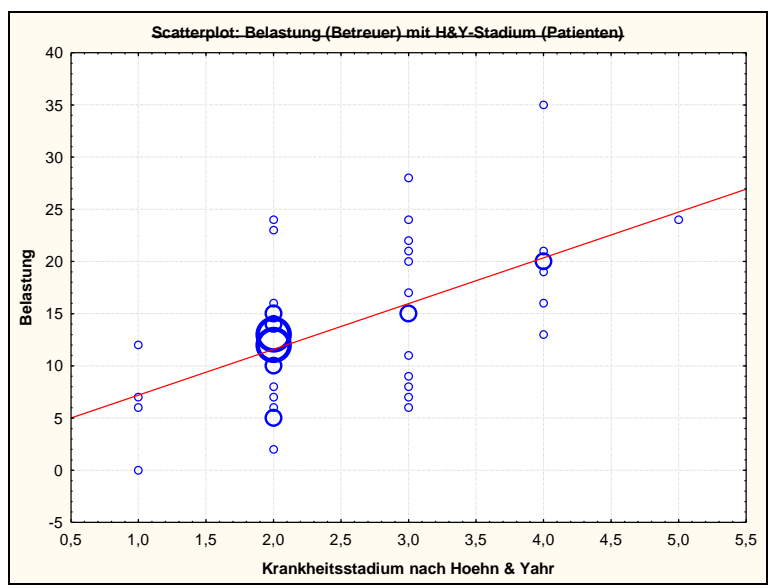

Abb. 72: Korrelation FAB und PSQI-Unterscore 5 (Betreuer)

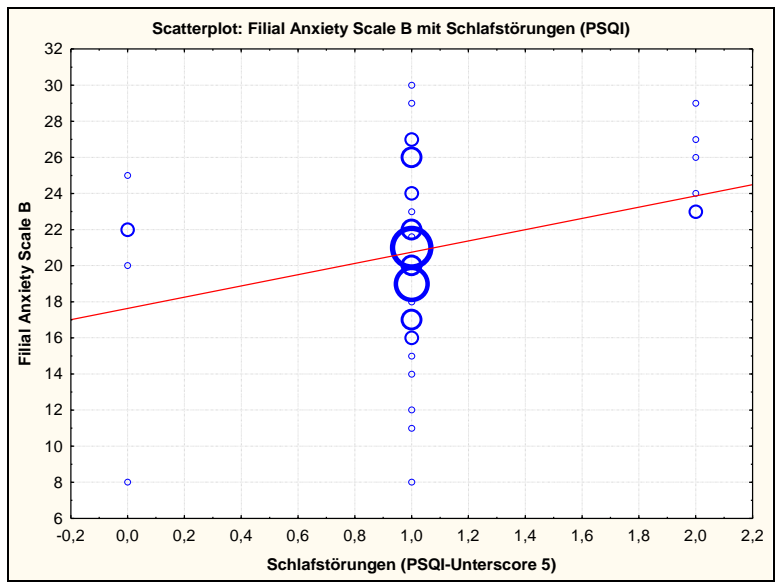




\section{Literaturverzeichnis}

- Adler C, Gunzelmann T, Machold C, Schumacher J, Wilz G (1996): Belastungserleben pflegender Angehöriger von Demenzpatienten. Z Gerontol Geriatr 29, 143 - 149

- Amirkhanyan AA, Wolf DA (2006): Parent care and the stress process: Findings from panel data. J Gerontol $\underline{61 B}$, S248 - S255

- Andrén S, Elmståhl S (2008): Psychosocial intervention for family caregivers of people with dementia reduces caregiver's burden: development and effect after 6 and 12 months. Scand J Caring Sci $\underline{22}, 98-109$

- Andresen EM, Malmgren JA, Carter WB, Patrick DL (1994): Screening for depression in well older adults: evaluation of a short form of the CES-D. Am J Prev Med 10, 77 - 84

- Antonini A, Barone P, Marconi R, Morgante L, Zappulla S, Pontieri FE, Ramat S, Ceravolo MG, Meco G, Cicarelli G (2012): The progression of non-motor symptoms in Parkinson's disease and their contribution to motor disability and quality of life. J Neurol $\underline{259}, 2621-2631$

- Årsland D, Larsen JP, Karlsen K, Lim NG, Tandberg E (1999): Mental symptoms in Parkinson's disease are important contributors to caregiver distress. Int J Geriatr Psychiatry 14, 866 - 874

- Backhaus J, Junghanns K, Broocks A, Riemann D, Hohagen F (2002): Test-retest reliability and validity of the Pittsburgh Sleep Quality Index in primary insomnia. J Psychosom Res $\underline{53}, 737$ 740

- Beaudreau SA, Spira AP, Gray HL, Depp CA, Long J, Rothkopf M, Gallagher-Thompson D (2008): The relationship between objectively measured sleep disturbances and dementia family caregiver distress and burden. J Geriatr Psychiatry Neurol 21, 159 - 165

- Bengtson VL, Martin P (2001): Families and intergenerational relationships in aging societies: comparing the United States with German-speaking countries. Z Gerontol Geriat 34, 207 - 217

- Blenkner M: Social work and family relationships in later life with some thoughts on filial maturity. In: Social structure and the family: Generational relations; hrsg. v. Shanas E, Streib GF, Prentice Hall, Englewood-Cliffs, NJ 1965, 46 - 59

- Bloch KE, Schoch OD, Zhang JN, Russi EW (1999): German version of the Epworth Sleepiness Scale. Respiration $\underline{66}, 440-447$

- Boddy F, Rowan EN, Lett D, O’Brien JT, McKeith IG, Burn DJ (2007): Subjectively reported sleep quality and excessive daytime somnolence in Parkinson's disease with and without dementia, dementia with Lewy Bodies and Alzheimer's disease. Int J Geriatr Psychiatry 22, 529 535

- Bosboom JLW, Stoffers D, Wolters EC (2004): Cognitive dysfunction and dementia in Parkinson's disease. J Neurol Transm $\underline{111}, 1303$ - 1315 
- Braak H, Rüb U, Braak E (2000): Neuroanatomie des Morbus Parkinson. Nervenarzt $\underline{71}, 459$ 469

- Brody EM (1981): "Women in the middle" and the family help to older people. Gerontologist 21, $471-480$

- Brody EM (1985): Parent care as a normative family stress. Gerontologist $\underline{25}, 19-29$

- Brody EM, Hoffman C, Kleban MH, Schoonover CB (1989): Caregiving dauthers and their local siblings: Perceptions, strains and interactions. Gerontologist $\underline{29}, 529-538$

- Bruder (1988): Filiale Reife - Ein wichtiges Konzept für die familiäre Versorgung kranker, insbesondere dementer alter Menschen. Z Gerontol Psychol Psychiatr 1, 95 - 101

- Brummett BH, Babyak MA, Siegler IC, Vitaliano PP, Ballard EL, Gwyther LP, Williams RB (2006): Associations among perceptions of social support, negative affect, and quality of sleep in caregivers and noncaregivers. Health Psychol 25, 220 - 225

- Bullinger M (1995): German translation and psychometric testing of the SF-36 Health Survey: preliminary results from the IQOLA Project. Soc Sci Med 41, 1359- 1366

- Bullinger M, Alonso J, Apolone G, Leplège A, Sullivan M, Wood-Dauphinee S, Gandek B, Wagner A, Aaronson N, Bech P, et al. (1998): Translating health status questionnaires and evaluating their quality: the IQOL Project approach. J Clin Epidemiol 51, 913 - 923

- Burke WJ, Roccaforte WH, Wengel SP (1991): The short form of the Geriatric Depression Scale: a comparison with the 30-item form. J Geriatr Psychiatry Neurol 4, 173 - 178

- Buysse DJ, Reynolds III CF, Monk TH, Berman SR, Kupfer DJ (1989): The Pittsburgh Sleep Quality Index: a new instrument for psychiatric practice and research. Psychiatry Res $\underline{28}, 193-$ 213

- Buysse DJ, Hall ML, Strollo PJ, Kamarck TW, Owens J, Lee L, Reis SE, Matthews KA (2008): Relationships between the Pittsburgh Sleep Quality Index (PSQI), Epworth Sleepiness Scale (ESS), and clinical/polysomnographic measures in a community sample. J Clin Sleep Med $\underline{4}, 563$ $-571$

- Caap-Ahlgren M, Dehlin O (2002): Factors of importance to the caregiver burden experienced by family caregivers of Parkinson's disease patients. Aging Clin Exp Res 14, 371 - 377

- Carpenter BD (2001): Attachment bonds between adult daughters and their older mothers: associations with contemporary caregiving. J Gerontol $\underline{56 \mathrm{~B}}, \mathrm{P} 257$ - P 266

- Carpenter JS, Andrykowski MA (1998): Psychometric evaluation of the Pittsburgh Sleep Quality Index. J Psychosom Res $\underline{45}, 5$ - 13

- Carter JH, Steward BJ, Archbold PG, Inoue I, Jaglin J, Lannon M, Rost-Ruffner E, Tennis M, McDermott MP, Amyot D et al. (1998): Living with a person who has Parkinson's disease: the spouses perspective by stage of disease. Mov Disord $\underline{13}, 20-28$

- Chappell NL, Reid RC (2002): Burden and well-being among caregivers: examining the distinction. Gerontologist $\underline{42}, 772-780$ 
- Chaudhuri KR, Pal S, DiMarco A, Whatley-Smith C, Bridgman K, Mathew R, Pezzela FR, Forbes A, Högl B, Trenkwalder C (2002): The Parkinson's disease sleep scale: a new instrument for assessing sleep and nocturnal disability in Parkinson's disease. J Neurol Neurosurg Psychiatry $\underline{73}$, $629-635$

- Chen H, Zhang SM, Schwarzschild MA, Hernán MA, Ascherio A (2006): Survival of Parkinson's disease patients in a large prospective cohort of male health professials. Mov Disord 21, $1002-$ 1007

- Cicirelli VG (1988): A measure of filial anxiety regarding anticipated care of elderly parents. Gerontologist $\underline{28}, 478-482$

- Cicirelli VG (1993): Attachment and obligation as daughters' motives for caregiving behavior and subsequent effect on subjective burden. Psychol Aging $\underline{8}, 144-155$

- Cicirelli VG (2000): An examination of the trajectory of the adult child's caregiving for an elderly parent. Fam Relat $\underline{49}, 169-175$

- Cifu DX, Carne W, Brown R, Pegg P, Ong J, Qutubuddin A, Baron MS (2006): Caregiver distress in parkinsonism. J Rehabil Res Dev $\underline{43}, 499$ - 508

- Collins NL, Feeney BC (2000): A safe haven: an attachment theory perspective on support seeking and caregiving in intimate relationships. J Pers Soc Psychol 모, $1053-1073$

- Comella CL (2003): Sleep disturbances in Parkinson's disease. Curr Neurol Neurosci Rep $\underline{3}, 173$ 80

- Comella CL (2007): Sleep disorders in Parkinson's disease: an overview. Mov Disord 22 (Suppl. 17), $S 367-S 373$

- Comella CL, Stebbins GT, Brown-Toms, Goetz CG (1994): Physical therapy and Parkinson's disease: a controlled clinical trial. Neurology $\underline{44}, 376-378$

- Creese J, Bédard M, Brazil K, Chambers L (2008): Sleep disturbances in spousal caregivers of individuals with Allzheimer's disease. Int Psychogeriatr 20, 149 - 161

- Crispi EL, Schiaffino K, Berman WH (1997): The contribution of attachment to burden in adult children of institutionalized parents with dementia. Gerontologist $37,52-60$

- Cupidi C, Realmuto S, Lo Coco G, Cinturino A, Talamanca S, Arnao V, Perini V, D’Amelio M, Savettieri G, Lo Coco D (2012): Sleep quality in caregivers of patients with Alzheimer's disease and Parkinson's disease and its relationship to quality of life. Int Psychogeriatr $\underline{24}, 1827-35$

- Daatland SO, Lowenstein A (2005): Intergenerational solidarity and the family-welfare state balance. Eur J Ageing 2, $174-182$

- Daire AP (2002): The influence of parental bonding on emotional distress in caregiving sons for a parent with dementia. Gerontologist $\underline{42}, 766-771$

- D'Amelio M, Ragonese P, Morgante L, Reggio A, Callari G, Salemi G, Savettieri G (2006): Long-term survival of Parkinson's disease. A population-based study. J Neurol 253, 33 - 37 
- Del Tredici K, Jost WH (2012): Gastrointestinale Störungen beim idiopathischen ParkinsonSyndrom. Nervenarzt $\underline{83}, 1282-1291$

- De Rijk MC, Launer LJ, Berger K, Breteler MMB, Dartigues JF, Baldereschi M, Fratiglioni L, Lobo A, Martinez-Lage J, Trenkwalder C et al. (2000): Prevalence of Parkinson's disease in Europe: A collaborative study of population-based cohorts. Neurology 54 (Suppl. 5), S21 - S23

- Dyck C (2009): Who cares fort he caregiver? Parkinsonism Relat Disord 15 (Suppl. 3), S118 - 21

- Edwards NE, Ruettiger KM (2002): The influence of caregiver burden on patients' management of Parkinson's disease: implications for rehabilitation nursing. Rehabil Nurs $\underline{27}, 182-186$

- Ellert U, Bellach BM (1999): Der SF-36 im Bundes-Gesundheitssurvey - Beschreibung einer aktuellen Normstichprobe. Gesundheitswesen $\underline{61}$ (Sonderheft 2), S184 - S190

- England M, Tripp-Reimer T (2003): Imminent concerns of filial caregivers reporting recent experience of crisis. Int J Aging Hum Dev 56, 67 - 88

- Etters L, Goodall D, Harrison BE (2008): Caregiver burden among dementia patient caregivers: A review of the literature. J Am Acad Nurse Pract 20, 423 - 428

- Fahn S, Elton RL, the UPDRS Development Committee: Unified Parkinson's Disease Rating Scale. In: Recent Events in Parkinson's Disease, Band 2. Hrsg. v. Fahn S, Marsden CD, Calne D, Goldstein M; Florham Park, New York 1987; 153 - 163

- Faison KJ, Faria SH, Frank D (1999): Caregivers of chronically ill elderly: Perceived burden. J Community Health Nurs $\underline{16}, 243-253$

- Fava M (2004): Daytime sleepiness and insomnia as correlates of depression. J Clin Psychiatry $\underline{65}$ (Suppl. 16), $27-32$

- Ferreira JJ, Desboeuf K, Galitzky M, Thalamas C, Brefel-Courbon C, Fabre N, Senard JM, Montastruc JL, Sampaio C, Rascol O (2006): Sleep disruption, daytime somnolence and "sleep attacks" in Parkinson's disease: A clinical survey in PD patients and age-matched volunteers. Eur J Neurol $\underline{13}, 209-214$

- Folstein MF, Folstein SE, McHugh PR (1975): "Mini-Mental State": A practical method for grading the cognitive state of patients for the clinician. J Psychiat Res $\underline{12}, 189-198$

- Franzen PL, Buysse DJ (2008): Sleep disturbances and depression: Risk relationships for subsequent depression and therapeutic implications. Dialogues Clin Neurosci $\underline{10}, 473-481$

- Funk L (2010): The interpretive dynamics of filial and collective responsibility for elderly people. Can Rev Sociol 47, $71-92$

- Funk L, Kobayashi KM (2009): „Choice“ in filial care work: Moving beyond a dichotomy. Can Rev Sociol 46, $235-252$

- Gentili A, Weiner DK, Kuchibhatla M, Edinger JD (1995): Test-retest reliability of the Pittsburgh Sleep Quality Index in nursing home residents (Letter). J Am Geriatr Soc 43, 1317 - 1318

- Glozman JM (2004): Quality of life of caregivers. Neuropsychol Rev 14, 183 - 196 
- Haenselt R, Danielzik A, Waack K (2004): Zur Evaluation von angeleiteten Gesprächsgrup-pen für pflegende Angehörige von Demenzkranken. Ergebnisse des GENA-Projektes in MecklenburgVorpommern. Z Gerontol Geriatr $\underline{37}, 402-410$

- Hagell P, Törnqvist AL, Hobart J (2008): Testing the SF-36 in Parkinson's disease_implications for reporting rating scale data. J Neurol $255,246-254$

- Hanagasi HA, Akat S, Gurvit H, Yazici J, Emre M (2011): Pain is common in Parkinson's disease. Clin Neurol Neurosurg $\underline{113}, 11-13$

- Happe S (2003): Excessive daytime sleepiness and sleep disturbances in patients with neurological diseases. Drugs $\underline{63}, 2725-2737$

- Happe S, Mayer G (2006): Schlaf bei neurodegenerativen Erkrankungen. Nervenarzt 77, 1251 1260

- Happe S, Berger K on behalf of the FAQT Study investigators (2002): The association between caregiver burden and sleep disturbances in partners of patients with Parkinson's disease. Age Aging 31, $349-354$

- Happe S, Berger K on behalf of the FAQT Study investigators (2003): The influence of poor sleep on quality of life in patients with Parkinson's disease - A prospective study. Somnologie $\underline{7}, 134-$ 139

- Happe S, Kungel M, Ebrecht M, Rink P, Modell S, Trenkwalder C (2004): Die Parkinson's Disease Sleep Scale (PDSS): Ein nützliches Instrument zur Quantifizierung von Schlafstörungen und zur Therapiekontrolle. Nervenheilkunde 23, $534-538$

- Havlikova E, Rosenberger J, Nagyova I, Middel B, Dubayova T, Gdovinova Z, van Dijk JP, Groothoof JW (2008): Impact of fatigue on quality of life in patients with Parkinson's disease. Eur J Neurol $\underline{15}, 475-480$

- Havlikova E, van Dijk JP, Nagyova I, Rosenberger J, Middel B, Dubayova T, Gdovinova Z, Groothoff JW (2011) J Neurol 258, 2222 - 2229

- Healy DG, Abou-Sleiman PM, Wood NW (2004): PINK, PANK, or PARK? A clinicians' guide to familial parkinsonism. Lancet Neurol $\underline{36}, 659-662$

- Herlofson K, Larsen JP (2003): The influence of fatigue on health-related quality of life in patients with Parkinson's disease. Acta Neurol Scand $\underline{107}, 1-6$

- Hoehn MM, Yahr MD (1967): Parkinsonism: onset, progression, and mortality. Neurology 17, $427-442$

- Hooker K, Manoogian-O'Dell M, Monahan DJ, Frazier LD, Shifren K (2000): Does type of disease matter? Gender differences among Alzheimer's and Parkinson's disease spouse caregivers. Gerontologist $\underline{40}, 568-573$

- Houlden H, Singleton AB (2012): The genetics and neuropathology of Parkinson's disease. Acta Neuropathol $\underline{124}, 325-338$ 
- Irwin M, Artin KH, Oxman MN (1999): Screening for depression in the older adult: Criterion validity of the 10-item Center for Epidemiological Studies Depression Scale (CES-D). Arch Intern Med $\underline{159}, 1701-1704$

- Jellinger KA (2012): Neuropathology of sporadic Parkinson's disease: Evaluation and Changes of Concepts. Mov Disord 27, 8 - 30

- Johns MW (1991): A new method for measuring daytime sleepiness: the Epworth Sleepiness Scale. Sleep $\underline{14}, 540-545$

- Jones PS, Lee JW, Zhang XE (2011): Clarifying and measuring filial concepts across five cultural groups. Res Nurs Health $\underline{34}, 310-326$

- Karantzas GC, Evans L, Foddy M (2010): The role of attachment in current and future parent caregiving. J Gerontol B Psychol Sci Soc Sci $\underline{65}, 573-580$

- Karlsen KH, Larsen JP, Tandberg E, Mæland JG (1999): Influence of clinical and demographic variables on quality of life in patients with Parkinson's disease. J Neurol Neurosurg Psychiatry $\underline{66}$, $431-435$

- Karlsen KH, Tandberg E, Årsland D, Larsen JP (2000): Health related quality of life in Parkinson's disease: a prospective longitudinal study. J Neurol Neurosurg Psychiatry $\underline{69}, 584-$ 589

- Khedr EM, El Fetoh NA, Khalifa H, Ahmed MA, El Beh KMA (2013): Prevalence of non motor features in a cohort of Parkinson's disease patients. Clin Neurol Neurosurg 115, 673 - 677

- Kim HS, Kim BJ, Kim KH, Choe MA, Yi M, Hah YS, Chung SJ, Kwon SH (2007): Subjective and objective caregiver burden in Parkinson's disease. Taehan Kanho Hakhoe Chi $\underline{37}, 242$ - 248

- Kochar J, Fredman L, Stone KL, Cauley JA for the Study of Osteoporotic Fractures (2007): Sleep problems in elderly women caregivers depend on the level of depressive symptoms: Results of the caregiver-study of osteoporotic fractures. J Am Geriatr Soc 55, 2003 - 2009

- Kohout FJ, Berkman LF, Evans DA, Cornoni-Huntley J (1993): Two shorter forms of the CES-D depression symptoms index. J Aging Health $\underline{5}, 179-193$

- Kuopio AM, Marttila RJ, Helenius H, Toivonen M, Rinne UK (2000): The quality of life in Parkinson's disease. Mov Disord 15, 216 - 223

- Kurz X, Scuvee-Moreau J, Vernooij-Dassen M, Dresse A (2003): Cognitive impairment, dementia and quality of life in patients and caregivers. Acta Neurol Belg 103, $24-34$

- Laditka SB, Pappas-Rogich M (2001): Anticipatory caregiving anxiety among older women and men. J Women Aging 13, 3 - 18

- Laux G: Affektive Störungen. In: Duale Reihe Psychiatrie und Psychotherapie. Hrsg. v. Bob A, Bob K; 3. Auflage ; Georg Thieme Verlag, Stuttgart 2005, 78 - 105

- Lee MA, Walker RW, Hildreth AJ, Prentice WM (2006): Individualized assessment of quality of life in idiopathic Parkinson's disease. Mov Disord 21, 1929 - 1934 
- Lees AJ, Blackburn NA, Campbell VL (1988): The nighttime problems of Parkinson's disease. Clin Neuropharmacol $\underline{11}, 512-519$

- Leitlinie Parkinson-Syndrome, Diagnostik und Therapie. Hrsg. v. Diener HC, Weimar C und der Deutschen Gesellschaft für Neurologie. Federführende Autoren Eggert KM, Oertel WH, Reichmann H. 5. Auflage; Thieme, Stuttgart 2012

- Lemke MR (2008): Depressive symptoms in Parkinson's disease. Eur J Neurol 15 (Suppl. 1), 21 25

- Linkersdörfer V: Einfluss der frühen Eltern-Kind-Bindung auf die Betreuung demenzkranker Menschen durch ihre erwachsenen Kinder. Med. Diss. Göttingen 2006

- Litvan I, Bhatia KP, Burn DJ, Goetz CG, Lang AE, McKeith I, Quinn N, Sethi KD, Shults C, Wenning GK (2003): SIC task force appraisal of clinical diagnostic criteria for parkinsonian disorders. Mov Disord 18, 467 - 486

- Lökk J (2008): Caregiver strain in Parkinson's disease and the impact of disease duration. Eur J Phys Rehabil Med 44, 39 - 45

- Lowenstein A (1999): Intergenerational family relations and social support. Z Gerontol Geriatr $\underline{32}$, $398-406$

- Lyness JM, Noel TK, Cox C, King DA, Conwell Y, Caine ED (1997): Screening for depression in elderly primary care patients. Arch Intern Med $\underline{157}, 449$ - 454

- Magai C, Cohen CJ (1998): Attachment styles and emotion regulation in dementia patients and their relation to caregiver burden. J Gerontol $\underline{53}$, P 147 - P 154

- Marcoen A: Adult children as caregivers to aging parents: A measure of filial maturity. Poster, präsentiert auf dem XV. Kongreß der International Association of Gerontology, Budapest, 4. - 9. Juli 1993

- Marcoen A (1995): Filial maturity of middle-aged children in the context of parent care: model and measures. J Adult Dev 2, 125 - 136

- Marcoen A (2005): Parent care: the core component of intergenerational relationships in middle and late adulthood. Eur J Ageing 2, $208-212$

- Markiewicz D, Reis M, Gold DP (1997): An exploration of attachment styles and personality traits in caregiving for dementia patients. Int J Aging Hum Dev $\underline{45}, 111$ - 132

- Marras C, McDermott MP, Rochon PA, Tanner CM, Naglie G, Lang AE, and the Parkinson Study Group DATATOP Investigators (2008): Predictors of deterioration in health-related quality of life in Parkinson's disease: Results from the DATATOP Trial. Mov Disord 23, 653 - 659

- Martínez-Martín P, Gil-Nagel A, Morlán Gracia L, Balseiro Gómez J, Martínez-Sarriés J, Bermejo F, the Cooperative Multicentric Group (1994): Unified Parkinson's Disease Rating Scale characteristics and structure. Mov Disord $\underline{9}, 76-83$ 
- Martínez-Martín P, Forjaz MJ, Frades-Payo B, Rusinol AB, Fernández-García JM, Benito-León J, Arillo VC, Barberá MA, Sordo MP, Catalán MJ (2007): Caregiver burden in Parkinson's disease. Mov Disord 22, $924-931$

- Martínez-Martín P, Arroyo S, Rojo-Abuin JM, Rodriguez-Blazquez C, Frades B, de Pedro Cuesta J, and the Longitudinal Parkinson's Disease Patient Study Group (2008): Burden, perceived health status, and mood among caregivers of Parkinson's disease patients. Mov Disord 23, $1673-1680$

- Masuhr KF, Neumann M: Parkinson-Krankheit; in: Duale Reihe Neurologie, 5. Auflage; hrsg. v. Bob A, Bob K; Georg Thieme Verlag, Stuttgart 2005, 199 - 207

- McCurry SM, Pike KC, Vitiello MV, Logsdon RG, Teri L (2008): Factors associated with concordance and variability of sleep quality in persons with Alzheimer's disease and their caregivers. Sleep $\underline{31}, 741-748$

- McHorney CA, Ware JE, Raczek AE (1993): The MOS 36-Item Short Form Health Survey (SF36): II. Psychometric and clinical tests of validity in measuring physical and mental health constructs. Med Care $\underline{31}, 247-263$

- McHorney CA, Ware JE, Lu JFR, Sherbourne CD (1994): The MOS 36-Item Short Form Health Survey (SF-36): III. Tests of data quality, scaling assumptions, and reliability across diverse patient groups. Med Care $\underline{32}, 40-66$

- McKibbin CL, Ancoli-Israel S, Dimsdale J, Archuleta C, von Kanel R, Mills P, Patterson TL, Grant I (2005): Sleep in spousal caregivers of people with Alzheimer's disease. Sleep 28, 1245 1250

- McLaughlin D, Hasson F, Kernohan WG, Waldron M, McLaughlin M, Cochrane B, Chambers H (2010): Living and coping with Parkinson's disease: Perceptions of informal carers. Palliat Med $\underline{25}, 177-182$

- Meara J, Mitchelmore E, Hobson P (1999): Use of the GDS-15 geriatric depression scale as a screening instrument for depressive symptomatology in patients with Parkinson's disease and their carers in the community. Age Ageing 28, 35 - 38

- Merschdorf U, Berg D, Csoti I, Fornadi F, Merz B, Naumann M, Becker G, Supprian T (2003): Psychopathological symptoms of Parkinson's disease compared to major depression. Psychopathology $\underline{36}, 221-225$

- Miyashita M, Narita Y, Sakamoto A, Kawada N, Akiyama M, Kayama M, Suzukamo Y, Fukuhara S (2009): Care burden and depression in caregivers caring for patients with intractable neurological diseases at home in Japan. J Neurol Sci $\underline{276}, 148$ - 152

- Molyneux GJ, McCarthy GM, McEniff S, Cryan M, Conroy RM (2008): Prvalence and predictors of carer burden and depression in carers of patients referred to an old age psychiatric service. Int Psychogeriatr 20, 1193 - 1202 
- Munhoz RP, Werneck LC, Teive HAG (2010): The differential diagnoses of parkinsonism: Findings from a cohort of 1528 patients and a 10 years comparison in tertiary movement disorders clinics. Clin Neurol Neurosurg $\underline{112}, 431-435$

- Murray PD, Lowe JD, Horne HL (1995): Assessing filial maturity through the use of the Filial Anxiety Scale. J Psychol 129, 519 - 529

- Murray PD, Lowe JD, Anderson HN, Horne HL, Lott WC, MacDonald S (1996): Validity study of the Filial Anxiety Scale. Gerontologist $\underline{36}, 110-112$

- Muslimović D, Post B, Speelman JD, Schmand B, de Haan RJ for the CARPA Atudy Group (2008): Determinants of disability and quality of life in mild to moderate Parkinson disease. Neurology $\underline{70}, 2241-2247$

- Nutt JG, Wooten GF (2005): Diagnosis and initial management of Parkinson's disease. N Eng J Med $\underline{353}, 1021-1027$

- Nydegger CN: The development of paternal and filial maturity; in: Parent-child relations throughout life; hrsg. v. Pillemer K, McCartney K; Lawrence Erlbaum Ass., Hillsdale, NJ 1991, $93-112$

- Ondo WG, Dat Vuong K, Khan H, Atassi F, Kwak C, Jankovic J (2001): Daytime sleepiness and other sleep disorders in Parkinson's disease. Neurology 57, 1392 - 1396

- O'Rourke N, Cappeliez P, Guindon S (2003): Depressive symptoms and physical health of caregivers of persons with cognitive impairment: analysis of reciprocal effects over time. J Aging Health $\underline{15}, 688-712$

- Pal PK, Thennarasu K, Fleming J, Schulzer M, Brown T, Calne SM (2004): Nocturnal sleep disturbances and daytime dysfunction in patients with Parkinson's disease and their caregivers. Parkinsonism Relat Disord $\underline{10}, 157$ - 168

- Parkinson J: An essay on the shaking palsy. Sherwood, Neely and Jones, London 1817

- Perrig-Chiello P, Sturzenegger M (2001): Social relations and filial maturity in middle-aged adults: contextual conditions and psychological determinants. Z Gerontol Geriatr $\underline{34}, 21-27$

- Perrig-Chiello P, Höpflinger F (2005): Aging parents and their middle-aged children: demographic and psychosocial challenges. Eur J Ageing 2, 183 - 191

- Phillips AC, Gallagher S, Hunt K, Der G, Carroll D (2009): Symptoms of depression on nonroutine caregivers: The role of caregiver strain and burden. Br J Clin Psychol 48 (Pt 4), 335 - 346

- Pinquart M, Sörensen S (2003): Differences between caregivers and noncaregivers in psychological health and physical health: A meta-analysis. Psychol Aging 18, $250-267$

- Poewe W (2008): Non-motor symptoms in Parkinson's disease. Eur J Neurol 15 (Suppl. 1), 14 20

- Quin Z, Zhang L, Sun F, Liu H, Fang X, Chan P, the Chinese Parkinson Study Group (2009): Depressive symptoms impacting on health-related quality of life in early Parkinson's disease: Results from Chinese L-dopa exposed cohort. Clin Neurol Neurosurg 111, 733 - 777 
- Radloff LS (1977): The CES-D Scale: a self-report depression scale for research in the general population. Appl Psychol Meas $\underline{1}, 385-401$

- Radloff LS, Teri L (1986): Use of the Center for Epidemiological Studies-Depression Scale with older adults. Clin Gerontol $\underline{5}, 119-136$

- Raschick M, Ingersoll-Dayton B (2004): The costs and rewards of caregiving among aging spouses and adult children. Fam Relat 53, $317-325$

- Raveis VH, Karus DG, Siegel K (1998): Correlates of depressive symptomatology among adult daughter caregivers of a parent with cancer. Cancer $\underline{83}, 1652-1663$

- Razali R, Ahmad F, Rahman FNA, Midin M, Sidi H (2011): Burden of care among caregivers of patients with Parkinson's disease: A cross-sectional study. Clin Neurol Neurosurg $\underline{113}, 639-643$

- Riazi A, Hobart JC, Lamping DL, Fitzpatrick R, Freeman JA, Jenkinson C, Peto V, Thompson AJ (2003): Using the SF-36 measure to compare the health impact of multiple sclerosis and Parkinson's disease with normal population health profiles. J Neurol Neurosurg Psychiatry $\underline{74}$, $710-714$

- Richards M, Marder K, Cote L, Mayeux R (1994): Interrater reliability of the Unified Parkinson's Disease Rating Scale motor examination. Mov Disord 9,89 - 91

- Ross BM, Mamalias N, Moszczynska A, Rajput A, Kish S (2001): Elevated activity of phospholipids biosynthetic enzymes in substantia nigra of patients with Parkinson's disease. Neuroscience 102, $899-904$

- Rowe MA, McCrae CS, Campbell JM, Benito AP, Cheng J (2008): Sleep pattern differences between older adult dementia caregivers and older adult noncaregivers using objective and subjective measures. J Clin Sleep Med $\underline{4}, 362$ - 369

- Savla J, Almeida DM, Davey A, Zarit SH (2008): Routine assistance to parents: Effects on daily mood and other stressors. J Gerontol 63B, S154 - S161

- Scaravilli T, Gasparoli E, Rinaldi F, Polesello G, Bracco F (2003): Health-related quality of life and sleep disorders in Parkinson's disease. Neurol Sci $\underline{24}, 209$ - 210

- Schmidt J: Filiale Reife und filiale Angst erwachsener Kinder demenzkranker Eltern. Med. Diss. Göttingen 2009

- Schrag A (2001): Medical and psychological determinants of quality of life in Parkinson's disease; in: $1^{\text {st }}$ International Symposium on Mental \& Behavioral Dysfunction in Movement Disorders. Mov Disord 16 (Suppl. 1), S1 - S13

- Schrag A, Jahanshahi M, Quinn N (2000): How does Parkinson's disease affect quality of life? A comparison with quality of life in the general population. Mov Disord 15, $1112-1118$

- Schrag A, Morley D, Quinn N, Jahanshahi M (2004): Impact of Parkinson's disease on patients' adolescent and adult children. Parkinsonism Relat Disord 10, 391 - 397 
- Schrag A, Hovris A, Morley D, Quinn N, Jahanshahi M (2006): Caregiver-burden in Parkinson's disease is closely associated with psychiatric symptoms, falls, and disability. Parkinsonism Relat Disord $\underline{12}, 35-41$

- Schütze Y (1995): Ethische Aspekte von Familien- und Generationenbeziehungen. Z Gerontopsychol-psychiatr $\underline{8}, 31-38$

- Schütze Y, Wagner M (1991): Sozialstrukturelle, normative und emotionale Determinanten der Beziehung zwischen erwachsenen Kindern und ihren alten Eltern. Z Sozialisationsforsch Erziehungssoziol 11, $295-313$

- Schulz JB, Gasser T: Parkinson-Syndrome; in: Therapie und Verlauf neurologischer Erkrankungen; hrsg. v. Brandt T, Dichgans J, Diener HC unter Mitarbeit namhafter Autoren; 5. Auflage; Verlag W. Kohlhammer, Stuttgart 2007, 961 - 1007

- Schulz R, O’Brien AT, Bookwala J, Fleissner K (1995): Psychiatric and physical morbidity effects of dementia caregiving: Prevalence, correlates, and causes. Gerontologist $\underline{35}, 771-791$

- Schwab JF, England AC: Projection technique for evaluating surgery in Parkinson's disease; in: Gillingham FJ, Donaldson MC: Third symposium on Parkinson's disease. Edinburgh: E\&S Livingstone 1969, $152-157$

- Schwarz B, Trommsdorff G (2005): The relation between attachment and intergenerational support. Eur J Ageing 2, $192-199$

- Sheik JI, Yesavage JA: Geriatric Depression Scale (GDS): Recent evidence and development of a shorter version. In: Clinical Gerontology: A Guide to Assessment and Intervention. Hrsg. v. Brink TL; Hawthorn Press, New York 1986; 165 - 173

- Shim B, Landerman LR, Davis LL (2011): Correlates of care relationship mutuality among carers of people with Alzheimer's and Parkinson's disease. J Adv Nurs $\underline{67}, 1729$ - 1738

- Shin H, Lee JY, Youn J, Kim JS, Cho JW (2012): Factors contributing to spousal and offspring caregiver burden in Parkinson's disease. Eur Neurol 67, 292 - 296

- Shpirer I, Miniovitz A, Klein C, Goldstein R, Prokhorov T, Theitler J, Pollak L, Rabey JM (2006): Excessive daytime sleepiness in patients with Parkinson's disease: A polysomnography study. Mov Disord 21, 1432 - 1438

- Silverstein M, Conroy SJ, Wang H, Giarrusso R, Bengtson VL (2002): Reciprocity in parent-child relations over the adult life course. J Gerontol 57B, S3 - S13

- Simuni T, Sethi K (2008): Nonmotor manifestations of Parkinson's disease. Ann Neurol $\underline{64}$ (Suppl.), S65 - S80

- Singleton AB, Farrer MJ, Bonifati V (2013): The genetics of Parkinson's disease: Progress an therapeutic implications. Mov Disord 28, $14-23$

- Sixel-Döring F, Trautmann E, Mollenhauer B, Trenkwalder C (2011): Associated factors for REM sleep behaviour disorder in Parkinson's disease. Neurology $\underline{77}, 1048$ - 1054 
- Slaughter JR, Slaughter KA, Nicholas D, Holmes SE, Martens MP (2001): Prevalence, clinical manifestations, etiology, and treatment of depression on Parkinson's disease. J Neuropsychiatry Clin Neurosci $\underline{13}, 187-196$

- Smith MC, Ellgring H, Oertel WH (1997): Sleep disturbances in Parkinson's disease patient and spouses. J Am Geriatr Soc 45, 194 - 199

- Spiegel R, Brunner C, Phil L, Ermini-Fünfschilling D, Monsch A, Notter M, Puxty J, Tremmel L (1991): A new behavioral assessment scale for geriatric out- and in-patiens: the NOSGER (Nurses' Observation Scale for geriatric Patients). J Am Geriatr Soc $\underline{39}$, 339 - 347

- Stebbins GT, Goetz CG (1998): Factor structure of the Unified Parkinson's Disease Rating Scale: motor examination section. Mov Disord 13, 633 - 636

- Stiens G: Untersuchung zum prädiktiven Wert der filialen Reife für die Betreuung demenzkranker alter Menschen durch ihre Kinder. Med. Diss. Göttingen 2000

- Svensson E, Beiske AG, Loge JH, Beiske KK, Sivertsen B (2012): Sleep problems in Parkinson's disease: A community-based staudy in Norway. BMC Neurol 12, 71

- Tandberg E, Larsen JP, Karlsen K (1999): Excessive daytime sleepiness and sleep benefit in Parkinson's disease: A community-based study. Mov Disord 14, 922 - 927

- Tarlov AR, Ware JE, Greenfield S, Nelson EC, Perrin E, Zubkoff M (1989): The Medical Outcome Study: an application of methods for monitoring the results of medical care. JAMA $\underline{262}$, $925-930$

- Tesch-Römer C (2001): Intergenerational solidarity and caregiving. Z Gerontol Geriat $\underline{34}, 28$ - 33

- Thommessen B, Årsland D, Braekhus A, Oksengaard AR, Engedal K, Laake K (2002): The psychosocial burden on spouses of the elderly with stroke, dementia and Parkinson's disease. Int J Geriatr Psychiatry $\underline{17}, 78-84$

- Twelves D, Perkins KSM, Counsell C (2003): Systematic review of incidence studies of Parkinson's disease. Mov Disord 18, 19 - 31

- Visser M, van Rooden SM, Verbaan D, Marinus J, Stiggelbout AM, van Hilten JJ (2008): A comprehensive model of health-related quality of life in Parkinson's disease. J Neurol 255, 1580 1587

- Visser M, Verbaan D, van Rooden SM, Marinus J, van Hilten JJ, Stiggelbout AM (2009): A longitudinal evaluation of health-related quality of life of patients with Parkinson's disease. Value Health $\underline{12}, 392-396$

- Vitaliano PP, Young HM, Zhang J (2004): Is caregiving a risk factor for illness? Curr Dir Psychol Sci $\underline{13}, 13-16$

- Voderholzer U, Al-Shajlawi A, Weske G, Feige B, Riemann D (2003): Are there gender differences in objective and subjective sleep measures? A study of insomniacs and healthy controls. Depress Anxiety 17, $162-172$ 
- Ware JE, Sherbourne CD (1992): The MOS 36-Item Short Form Health Survey (SF-36): I. Conceptual framework and item selection. Med Care $\underline{30}, 473$ - 483

- Weintraub D, Xie S, Karlawish J, Siderowf A (2007): Differences in depression symptoms in patients with Alzheimer's and Parkinson's disease: Evidence from the 15-item Geriatric Depression Scale (GDS-15). Int J Geriatr Psychiatry 22, 1025 - 1030

- Wilcox S, King AC (1999): Sleep complaints in older women who are family caregivers. J Gerontol 54B, P189 - P198

- Willette-Murphy K, Todero C, Yeaworth R (2006): Mental health and sleep of older wife caregivers for spouses with Alzheimer's disease and related disorders. Issues Ment Health Nurs. $\underline{27}, 837-852$

- Wilz G, Adler C, Gunzelmann T, Brähler E (1999): Auswirkungen chronischer Belastungen auf die physische und psychische Befindlichkeit - Eine Prozessanalyse bei pflegenden Angehörigen von Demenzkranken. Z Gerontol Geriatr $\underline{32}, 232-250$

- Wüllner U, Schmitz-Hübsch T, Antony G, Fimmers R, Spottke A, Oertel WH, Deuschl G, Klockgether T, Eggert K on behalf of the KNP e.V. (2007): Short Communication: Autonomic dysfunction in 3414 Parkinson's disease patients enrolled in the German Network of Parkinson's disease (KNP e.V.): The effect of ageing. Eur J Neurol 14, 1405 - 1408

- Yee JL, Schulz R (2000): Gender differences in psychiatric morbidity among family caregivers: a review and analysis. Gerontologist $\underline{40}, 147-164$

- Yesavage JA, Brink TL, Rose TL, Lum O, Huang V, Adey M, Leirer VO (1983): Development and validation of a geriatric depression screening scale: a preliminary report. J Psychiat Res $\underline{17}, 37$ $-49$

- Yoshioka E, Saijo Y, Kita T, Satoh H, Kawaharada M, Fukui T, Kishi R (2012): Gender differences in insomnia and the role of paid work and family responsibilities. Soc Psychiatry Psychiatr Epidemiol 47, 651 - 662

- Zarit SH, Reever KE, Bach-Peterson (1980): Relatives of the impaired elderly: correlates of feelings of burden. Gerontologist 20, $649-655$

- Zeitlhofer J, Schmeiser-Rieder A, Tribl A, Rosenberger A, Bolitschek J, Kapfhammer G, Saletu B, Katschnig H, Holzinger B, Popovic R, Kunze M (2000): Sleep and quality of life in the Austrian population. Acta Neurol Scand 102, $249-257$

- Zhan HJ (2005): Social-economic context of parent care: Explaining Chinese caregivers' psychological and emotional well-being. J Gerontol Soc Work $\underline{45}, 83-100$

- Zhaohui Q, Zhang L, Sun F, Hongjun L, Fang X, Chan P, the Chinese Parkinson Study Group (2009): Depressive symptoms impacting on health-related quality of life in early Parkinson's disease: Results from Chinese L-Dopa exposed cohort. Clin Neurol Neurosurg 111, 733 - 737 


\section{Danksagung}

An dieser Stelle möchte ich mich recht herzlich bei all denen bedanken, die mich bei der Erstellung meiner Doktorarbeit auf unterschiedliche Art und Weise unterstützt haben. Ich danke meiner Doktormutter Frau Prof. Dr. med. Svenja Happe und meiner Betreuerin Frau Dr. med. Gerthild Stiens für die Unterstützung dieser Arbeit. Neben der geduldigen Beantwortung etlicher Fragen danke ich ihnen insbesondere für ihre Geduld bezüglich des „,protrahierten Verlaufs“ der Fertigstellung der schriftlichen Fassung. In Sachen Patientenrekrutierung danke ich den Mitarbeitern der Parkinsonambulanz der Abteilung für Klinische Neurophysiologie des Universitätsklinikums Göttingen unter der Leitung von Prof. Dr. med. Walter Paulus und den Mitarbeitern der ParacelsusElena-Klinik in Kassel unter der Leitung von Frau Prof. Dr. med. Claudia Trenkwalder sowie den beiden niedergelassenen Neurologen in Wolfenbüttel und Göttingen. Darüber hinaus danke ich den Mitarbeitern der Abteilung Medizinische Statistik für ihre geduldige Beratung bei der Auswertung.

Mein besonderer Dank gilt zudem den Pakinsonpatienten und ihren Partnern sowie ihren erwachsenen betreuenden Söhnen und Töchtern, die sich freundlicherweise die Zeit nahmen, an dieser Untersuchung teilzunehmen. Ich danke auch allen Kontrollpersonen für ihre Mühen und denjenigen, die mir bei deren Rekrutierung maßgeblich halfen. 